\title{
Expanding the Applications of Ion Mobility Spectrometry and Mass Spectrometry in Integrative 'Omics Analyses
}

\author{
Gregory C. Donohoe
}

Follow this and additional works at: https://researchrepository.wvu.edu/etd

\section{Recommended Citation}

Donohoe, Gregory C., "Expanding the Applications of Ion Mobility Spectrometry and Mass Spectrometry in Integrative 'Omics Analyses" (2016). Graduate Theses, Dissertations, and Problem Reports. 5505.

https://researchrepository.wvu.edu/etd/5505

This Dissertation is protected by copyright and/or related rights. It has been brought to you by the The Research Repository @ WVU with permission from the rights-holder(s). You are free to use this Dissertation in any way that is permitted by the copyright and related rights legislation that applies to your use. For other uses you must obtain permission from the rights-holder(s) directly, unless additional rights are indicated by a Creative Commons license in the record and/ or on the work itself. This Dissertation has been accepted for inclusion in WVU Graduate Theses, Dissertations, and Problem Reports collection by an authorized administrator of The Research Repository @ WVU.

For more information, please contact researchrepository@mail.wvu.edu. 


\title{
Expanding the Applications of Ion Mobility Spectrometry and Mass Spectrometry in Integrative 'Omics Analyses
}

\author{
Gregory C. Donohoe \\ Dissertation submitted to the \\ Eberly College of Arts and Sciences \\ at West Virginia University \\ in partial fulfillment of the requirements \\ for the degree of \\ Doctor of Philosophy \\ In \\ Analytical Chemistry \\ Approved by \\ Stephen J. Valentine, Ph.D., Committee Chairperson \\ Lisa A. Holland, Ph.D. \\ Fred L. King, Ph.D. \\ Brian V. Popp, Ph.D. \\ Andrew K. Shiemke, Ph.D. \\ C. Eugene Bennett Dept. of Chemistry \\ Morgantown, West Virginia \\ 2016
}

Key Terms: ion mobility, mass spectrometry, hydrogen deuterium exchange (HDX), 'omics, electron transfer dissociation, parallel dissociation, collision induced dissociation, charge transfer dissociation, metabolomcis, phosphoproteomics, proteomics, ubiquitin,

Copyright 2016 Gregory C. Donohoe 


\title{
ABSTRACT
}

\section{Expanding the Applications of Ion Mobility Spectrometry and Mass Spectrometry in Integrative 'Omics Analyses}

\author{
Gregory C. Donohoe
}

Over the past few decades, biomolecular analyses ranging from the study of complex mixtures to protein structural interrogation have increased significantly. These studies range from small molecule separations[1,2] to observing structural trends in large proteins and protein sub-complexes.[3,4] Traditionally, the use of liquid chromatography mass spectrometry (LC-MS), electrophoresis and nuclear magnetic resonance (NMR) spectroscopy have been at the forefront of these respective studies. Because complex mixtures can contain a variety of components over a wide dynamic range and proteins and their complexes can contain a diverse array of structures, few analytical techniques are capable of providing information across all experimental areas (e.g. small molecule mixtures to large individual proteins). In contrast, the use of lon Mobility Spectrometry-Mass Spectrometry (IMS-MS) has emerged as a powerful tool for measuring ion(s) structural heterogeneity. While IMS-MS is a relatively newer method, workflows are becoming more common as the commercialization of IMS instruments has created a larger user base. Such workflows now include metabolomic, $[1,5,6]$ lipidomic,[7] proteomics and protein structural analyses[8, 9]. Taken collectively, these areas encompass the field of 'omics' analysis. While each field has its respective difficulties, IMS-MS is well poised to enhance and even expand the repertoire of analytical platforms for omics analyses.

Much of the current bottlenecks in traditional techniques suffer from an inability to sample measureable species rapidly in a reproducible manner over a wide dynamic range. For example, Anderson and coworkers have proposed that the plasma proteome includes $10^{6}-10^{7}$ species that span a concentration range of $10^{11}$.[10] In many cases, IMS has shown improved resolution of isomeric species compared to either LC or Gas Chromatography (GC) analyses.[11, 12] The utility of IMS-MS in profiling is largely attributed to its rapid ability to resolve low-abundance species from spectral regions containing high-abundance species, thereby increasing measurement sensitivity, dynamic range and peak capacity.[13-17] Additionally, IMS is capable of separating isobaric species that cannot be resolved by MS alone. In 'omic profiling directed toward biomarker discovery, it is imperative to identify compounds of interest. The identification is complicated by compound diversity (class and structural variation).

Traditionally, as well as all commercially available, IMS-MS instruments use Time-of-Flight (ToF) mass analyzers for determining an ion's mass-to-charge ratio $(\mathrm{m} / \mathrm{z})$. The obvious advantage is the ability to nest the $\mathrm{m} / \mathrm{z}$ measurement $(\mu \mathrm{s})$ within the drift measurement (ms). This creates an orthogonal separation where many $\mathrm{m} / \mathrm{z}$ measurements are made during the drift separation. Although this combination creates a rapid, multidimensional analysis, ToF mass analyzers are not capable of multistage 
tandem mass spectrometry $\left(\mathrm{MS}^{\mathrm{n}}\right)$ or nonergodic dissociation methods such as electron transfer dissociation (ETD). These MS fragmentation methods are often used as standalone techniques in applications ranging from small molecule identification within complex mixtures to identifying high order structure in proteins using Hydrogen Deuterium exchange (HDX) MS. To this end, new applications of IMS-MS that leverage the use of ion trapping MS are useful for supplementing these limitations of ToF analyzers. Trapping mass analyzers add the capability to perform ion-neutral or ion-ion reactions on drift-selected ions. In such experiments, fragment ions are generated and are structurally useful in identifying and quantifying individual components or those that compose protein structures or post translational modifications (PTMs). To date, very few, if any, experiments have attempted to combine the unique capabilities of IMS-MS with $M S^{n}$ or ETD-MS for uncovering ion structural information or heterogeneity. As will be shown in the coming chapters, coupling IMS to trapping mass analyzers expands the capabilities into new areas of 'omics analysis and enhances the information that can be obtained from either technique alone. 
1. Dwivedi, P., et al., Metabolic profiling by ion mobility mass spectrometry (IMMS). Metabolomics, 2008. 4(1): p. 63-80.

2. Valentine, S.J., et al., Gas-phase separations of protease digests. Journal of the American Society for Mass Spectrometry, 1998. 9(11): p. 1213-1216.

3. Ruotolo, B.T., et al., Evidence for macromolecular protein rings in the absence of bulk water. Science, 2005. 310(5754): p. 1658-1661.

4. van Duijn, E., et al., Native tandem and ion mobility mass spectrometry highlight structural and modular similarities in clustered-regularly-interspaced shot-palindromic-repeats (CRISPR)associated protein complexes from Escherichia coli and Pseudomonas aeruginosa. Mol Cell Proteomics, 2012. 11(11): p. 1430-41.

5. Zhang, X., et al., Metabolic Analysis of Striatal Tissues from Parkinson's Disease-like Rats by Electrospray Ionization Ion Mobility Mass Spectrometry. Analytical Chemistry, 2014.

6. Paglia, G., et al., Ion Mobility Derived Collision Cross Sections to Support Metabolomics Applications. Analytical Chemistry, 2014. 86(8): p. 3985-3993.

7. Kliman, M., J.C. May, and J.A. McLean, Lipid analysis and lipidomics by structurally selective ion mobility-mass spectrometry. Biochimica et Biophysica Acta (BBA) - Molecular and Cell Biology of Lipids, 2011. 1811(11): p. 935-945.

8. Zhuang, X., et al., Identification of Unfolding and Dissociation Pathways of Superoxide Dismutase in the Gas Phase by Ion-Mobility Separation and Tandem Mass Spectrometry. Analytical Chemistry, 2014. 86(23): p. 11599-11605.

9. Allen, S.J., A.M. Schwartz, and M.F. Bush, Effects of polarity on the structures and charge states of native-like proteins and protein complexes in the gas phase. Anal Chem, 2013. 85(24): p. 12055-61.

10. Anderson, N.L., et al., The human plasma proteome: a nonredundant list developed by combination of four separate sources. Mol Cell Proteomics, 2004. 3(4): p. 311-26.

11. Wu, C., et al., Separation of isomeric peptides using electrospray ionization/high-resolution ion mobility spectrometry. Anal Chem, 2000. 72(2): p. 391-5.

12. Dwivedi, P., et al., Rapid Resolution of Carbohydrate Isomers by Electrospray lonization Ambient Pressure Ion Mobility Spectrometry-Time-of-Flight Mass Spectrometry (ESI-APIMS-TOFMS). Journal of the American Society for Mass Spectrometry, 2007. 18(7): p. 1163-1175.

13. Valentine, S.J., et al., Toward Plasma Proteome Profiling with Ion Mobility-Mass Spectrometry. Journal of Proteome Research, 2006. 5(11): p. 2977-2984.

14. Zinnel, N.F., P.J. Pai, and D.H. Russell, Ion Mobility-Mass Spectrometry (IM-MS) for Top-Down Proteomics: Increased Dynamic Range Affords Increased Sequence Coverage. Analytical Chemistry, 2012. 84(7): p. 3390-3397. 
15. Dwivedi, P., A.J. Schultz, and H.H. Hill, Metabolic profiling of human blood by high-resolution ion mobility mass spectrometry (IM-MS). International Journal of Mass Spectrometry, 2010. 298(13): p. 78-90.

16. Ruotolo, B.T., et al., Peak capacity of ion mobility mass spectrometry: Separation of peptides in helium buffer gas. Journal of Chromatography B-Analytical Technologies in the Biomedical and Life Sciences, 2002. 782(1-2): p. 385-392.

17. Counterman, A.E., et al., Formation of peptide aggregates during ESI: Size, charge, composition, and contributions to noise. Journal of the American Society for Mass Spectrometry, 2001. 12(9): p. 1020-1035. 


\section{Dedication}

I dedicate this work to my wife, Allyson and unborn son, Cole. You have made this work worth doing and you are both my inspiration. I thank my wife for her patients and willingness to supporting my decisions throughout my graduate career. You have made me a better man. My goal is to provide our family with a comfortable life.

\section{Acknowledgements}

I would like to express my deepest gratitude towards every individual who has served as a mentor over my chemistry career: Dr. Matthew Scanlon and Dr. Andreas Baur for introducing me to chemistry and giving me a scientific foundation from which to grow; Ahmad Rhaman for his scientific insight, direction and conversation; Dr. Lisa Holland for her most valued mentorship and high expectations. Your advice and teachings have changed my perception of science and my views on how to be a scientist. Thank you for always taking the time to share your wisdom both scientifically and personally; Dr. Fred King for getting me started with mass spectrometry. Words can't express how thankful I am to have taken your mass spectrometry class. You have undoubtedly introduced me to one of my greatest interests in life - thank you; Dr. Brian Popp for being one of the most knowledgeable scientists l've met. Your scientific understanding is rich and always ignites great discussions and future directions - I truly admire that; Dr. Andrew Shiemke for agreeing to be a part of my graduate career and always offering fruitful discussions about biochemistry and protein structure; and Dr. Stephen Valentine for having to work with my strengths and weakness. Thank you for your willingness to teach me your expertise and the patients to answer every question. I am beyond thankful that I had the opportunity to learn from someone I very much respect. I am most sincerely grateful that you were my academic mentor. 
I would also like to thanks my peers, Jim Arndt, Brian Nichols, Hossein Maleki, Mahdiar Khakenejad, Samaneh Kondalaji and Megan Maurer. I think we've all grown together and been presented the unique opportunity to learn from one another. l've truly enjoyed my time with you all. I wish each one of you success in all of your challenges and scientific opportunities. I believe my memories in the Valentine group and my graduate career at WVU will last a lifetime. 


\section{TABLE OF CONTENTS}

Abstract

Dedication $\quad$ vi

Acknowledgements $\quad$ vi

Table of Contents viii

Appendices $\quad$ xiv

List of Figures $\quad$ XV

List of Tables $\quad$ xvii

List of Symbols and Abbreviations xvii

1. Introduction: Applications of Ion Mobility Spectrometry and Mass 1 Spectromety in 'Omic Analyses

1.1. Fundamentals of Ion Mobility Spectrometry (IMS) 1

1.2. Fundamentals of Mass Spectrometry (MS) - Linear lon Traps 8

1.3. Collision Induced Dissociation (CID) in Trapping Mass Analyzers 13

1.3.1. CID of Peptides and Proteins: $b$ - and $y$-ions 13

1.3.2. CID of Small Molecules: Understanding Fragmentation 14

1.4. IMS-MS Metabolomics and Small Molecule Studies 15

1.5. An Overview of the Metablome and Traditional Techniques 16

1.5.1. Parallel Dissociation Combined with IM-MS for Data 18 Independent Analysis of Small Molecules

1.6. Electron Transfer Dissociation (ETD) of Peptides and Proteins 20

1.6.1. ETD Mechanism: $c$ - and $z-$ ions 21

1.6.2. IMS - ETD - MS Techniques 22

1.7. Phosphoproteomics and MS Techniques 23 
1.7.1. Coupling IM-CID-ETD-MS for Determining the Presence and Location of Phosphorylation from Complex Mixtures.

1.8. Solution Hydrogen Deuterium Exchange: Fundamentals 26

1.8.1. Bottom - up Hydrogen Deuterium Exchange - Mass 28 Spectrometry (HDX - MS)

1.8.2. Top - down HDX - MS 29

1.8.2.1. Pre - residue Deuterium Measurements 30

1.8.3. Solution HDX Coupled with IMS - ETD - MS 31

1.9. Gas Phase Hydrogen Deuterium Exchange Coupled with IMS-MS 32

1.10. Helium Charge Transfer Dissociation 35

$\begin{array}{ll}\text { 1.11. References } & 37\end{array}$

2. A New Ion Mobility - Linear Ion Trap for Complex Mixture Analysis 47

2.1. Introduction: Complex Mixture Separations Using IMS-MS 47

2.2. Materials and Methods $\quad 50$

2.2.1. Phosphoproteomic Samples $\quad 50$

2.2.2. Rat Exposure to $\mathrm{TiO}_{2}$ Nanoparticles 50

2.2.2.1. Metabolite Extraction $\quad 51$

2.2.3. IMS-MS: Instrumental Operation 52

2.2.3.1. Recording Two - Dimensional (2D) $t_{D}(\mathrm{~m} / \mathrm{z})$

Distributions

2.2.3.2. Parallel Dissociation 53

2.2.4. Mass Spectrometry Measurements 54

2.2.5. Generating IMS-MS Datasets $\quad 55$

2.2.5.1. Comparing IMS-MS Datasets 55 
2.2.6. Detection Limit Sample Preparation

2.2.7. Dynamic Range Sample Preparation 56

2.2.8. Principal Component Analysis (PCA) 56

2.2.9. Exact Mass Measurements 57

2.3. Results and Discussion $\quad 57$

2.3.1. Instrument Figures of Merit 57

2.3.1.1. Detection Limit Studies 58

2.3.1.2. Dynamic Range Studies 59

$\begin{array}{ll}\text { 2.3.2. Phosphopeptide Analysis } & 60\end{array}$

2.3.2.1. Aligning Extracted lon Drift Time Distributions 61 (XIDTDs)

2.3.2.2. IMS - CID - ETD - MS of Precursor and Fragment 61 ions

2.3.3. A Comparative IMS - MS Analysis of Metabolimic Samples 64

2.3.3.1. PCA of Sample Cohorts 66

2.3.3.2. Comparing Extraction Ion Drift Time Distributions 67 (XIDTDs)

2.3.3.3. IMS - MS ${ }^{n}$ Experiments for Metabolomic Analysis $\quad 70$

2.3.3.4. IMS - CID - MS Measurements 72

2.2.4. Instrumental Implication for Comparative Analsyses 77

$\begin{array}{ll}\text { 2.4.. References } & 78\end{array}$

3. Negative ion IMS-HDX-MS

3.1. Introduction: A Base for Studying Anionic Peptides and Proteins 82

3.2. Method and Materials 83

3.2.1. Sample Preparation 83 
3.2.2. IMS-MS: Instrumental Operation

3.2.2.1. Recording Two - Dimensional (2D) $t_{D}(\mathrm{~m} / \mathrm{z})$

Distributions

3.2.3. Mass Spectrometry Measurements

3.2.4. Generating IMS-MS Datasets 86

3.2.5. Gas Phase HDX Experiment Design 86

3.2.5.1. HDX Levels and Rate Constants 87

$\begin{array}{ll}\text { 3.3. Results and Discussion } & 87\end{array}$

3.3.1. Protein Anion Collision Cross Sections 87

3.3.2. Gas - Phase Deuterium Uptake as a Function of $\mathrm{D}_{2} \mathrm{O} \quad 90$ Pressure

3.3.2.1. Anion HDX levels 93

3.3.2.2. HDX Kinetics of Deprotonated Peptides and 95 Proteins

3.3.3. Structural Implications of the HDX Results 96

3.3.4.1. Exchange Efficiency and the Protein Fold - Ubiquitin 97

3.3.4. Experimental Limitations 101

3.3.5. Conclusions 102

$\begin{array}{ll}\text { 3.3.6. References } & 104\end{array}$

4. On - line Deuterium Hydrogen Exchange and Pepsin Digestion Coupled 109 to IMS - MS and Tandem Mass Spectrometry

4.1. Introduction: Deuterium Hydrogen Exchange and IMS - MS 109

4.2. Methods and Materials 112

4.2.1. Sample Preparation 112

4.2.2. On - Line DHX - PD - IMS System 112 
4.2.3. Scrambling Control On - Line System

4.2.3.1. Optimized Parameters

4.2.3. IMS-MS: Instrumental Operation

4.2.3.1. Recording Two - Dimensional (2D) $t_{D}(\mathrm{~m} / \mathrm{z})$ Distributions

4.2.4. Mass Spectrometry Measurements

4.2.4.1. CID - MS Experiments

4.2.4.1.1. Identification of Precursor lons

4.2.4.2. Deuterated Samples and ETD Analysis

4.2.4.3. IMS - MS Datasets

4.2.4.3. Comparing XIDTDs for Identification

4.2.4.4. Per - Residue Deuterium Measurements

4.3. Results and Discussion

4.3.1. Comparing Peptides and 2D IMS - MS Datasets

4.3.2. Generating XIDTDs and Combing CID for Unlabeled Peptide 121 Identification

4.3.3. Matching XIDTDs Between Undeuterated and Deuterated

Datasets

4.3.4. Overall Per - Peptide Deuterium Analysis - Ubiquitin

4.3.5. Scarmbling Control Peptide Studies

4.3.6. IMS - ETD -MS of Deuterated Peptides for Per - Residue Analysis - Ubiquitin

4.3.7. Reproducibility of the On - Line DHX-PD-IMS-MS System

4.3.8. Assessment of the Effects of ESI Solvent Composition 
5. Helium Charge Transfer Dissociation Combined with Deuterium Hydrogen Exchange Mass Spectrometry

5.1. Introduction: Fragmentation Techniques and Hydrogen Deuterium Scrambling

5.2. Method and Materials

5.2.1. Sample Preparation

5.2.2. Modified Instrument

5.2.3. HD - Scrambling Peptide

5.2.4. Mass Spectrometry Measurements and Parameters

5.2.4.1. ETD Measurements

5.2.4.2. He-CTD Measurements

5.2.5. Per-Residue Deuterium Measurements

5.3. Results and Discussion

5.3.1. Peptide Control Studies and HD - Scrambling Evaluation

5.3.1.1. He-CTD HD-Scrambling Analysis

5.3.1. He - CTD Mechanistic Insights for $c$ - ions

5.3.2. Ammonia Neutral Loss and $\mathrm{N}$ - terminal Scrambling

5.3.3. $\mathrm{DHX}-\mathrm{He}-\mathrm{CTD}-\mathrm{MS}$ Structural Determinations

5.3.3.2. Per - Residue He - CTD - MS Analysis

5.5. References 
6.1. Post Translational Modifications: Phosphorylation

6.1.1. Doubly - Phosphorylated Peptides from Complex Mixtures

6.1.2. Conducting IMS-CID-MS on Glycosylated Proteins

6.2. Expanding the Usage of IMS-CID-MS in Metabolomic Workflows

6.3. Gas Phase HDX of Anionic Proteins

172

6.3.1. Comparing Cationic and Anionic HDX Deuterium Level: Structural Studies

6.3.2. Binding Faces of Acidic Proteins Involved in Protein Aggregates

6.3.3. Gas Phase HDX and Anionic Glycans: Structural Considerations and Separation Techniques

6.4. Solution - Base DHX Coupled with IMS-ETD-MS

6.4.1. Constructing a New Micro-fluidic Chip of On - Line Analysis

6.4.2. Increasing Sequence Coverage for Per-residue Studies

6.4.3. Evaluating Larger Proteins

6.4.4. Connecting Rapid Solution Structural Changes with Solution 177 DHX and IMS-MS

6.5. Helium Charge Transfer Dissociation : Insights and Future Outlook

6.5.1. Using $\mathrm{He}-\mathrm{CTD}$ for Anionic Peptides and Proteins 178

6.5.2. Evaluating Other lons for HD-Scrambling 179

6.5.3. Other Potential Usages; IMS - He - CTD - MS 179

6.6. References

\section{Appendices}

Appendix 1. Optimized Instrumental Parameters for DHX-IMS-ETD-MS 


\section{LIST OF FIGUES}

1.1. Schematic of the ion mobility linear ion trap 2

2.1.Mass spectrum of model peptide $[\mathrm{M}+3 \mathrm{H}]^{3+}$

2.2. XIDTD of Cytochrom c Tryptic Peptide $m / z 806.5$

2.3. Two-dimensional (2D) $t_{D}(\mathrm{~m} / \mathrm{z})$ heat-plot of a cytochrome $c$ tryptic digest 62 containing the phosphorylated peptide standard [KRPsQRHGSKY-NH ${ }_{2}$

2.4. (A) Mobility - selected IMS-CID-ETD-MS spectra of [KRPsQRHGSKY$\left.\mathrm{NH}_{2}+3 \mathrm{H}\right]^{3+}$ prescursor and(B) Neutral loss fragment ion

2.5. $t_{D}(m / z)$ heat-plot of the EXP24 metabolite extract 65

2.6.(A) PCA plot of all sample cohorts (B) loadings matrix values for metabolite $\quad 67$ ions

2.7. (A) XIDTDs comparison of $\mathrm{m} / \mathrm{z} 518.3$ and (B) 732.2 for Exposed 24 for Control 24 sample cohorts

2.8. Multistage tandem mass spectrometry of $\mathrm{m} / z 518.3$ and 542.3

2.9. $t_{D}(m / z)$ heat-plot of the EXP24 sample obtained from an IMS-CID-MS 73 experiment.

2.10. Matching XIDTDs of fragment ions to precursor ions 74

2.11. semi-quantiative assessment for matching XIDTDs from IMS-CID-MS 76 experiments

3.1. (A) Two-dimensional, $t_{D}(\mathrm{~m} / \mathrm{z})$ dot plot of electrosprayed ubiquitin ions and 88 (B) , $t_{D}(\mathrm{~m} / \mathrm{z})$ dot plot of electrosprayed ubiquitin ions at a partial pressure of 0.04 torr $\mathrm{D}_{2} \mathrm{O}$

3.2. (A)Collision Cross Section for protein and (B) insulin anions

3.3. (A) Plot of deuterium uptake and (B) Plot of the natural log of the number of remaining exchanged residues. Both are plotted against the product of the partial pressure $\mathrm{D}_{2} \mathrm{O}$ and drift time

3.4. Plot of the maximum HDX level for protein anionic conformers

3.5. Expanded Two-dimensional, $t_{D}(\mathrm{~m} / \mathrm{z})$ heat plot of the $[\mathrm{M}-6 \mathrm{H}]^{6-}$ charge state of 
ubiquitin after gas phase $\mathrm{HDX}$ with $\mathrm{D}_{2} \mathrm{O}$ at a partial pressure of 0.04 torr.

3.6. Plot of cross section/maximum exchange level for protein charge states 100

4.1. Schmatic representation of the on-line system for DHX-PD-IMS-MS 113

4.2. (A) Two-dimensional (2D) $t_{D}(\mathrm{~m} / \mathrm{z})$ raised-relief plot showing the resulting 120 peptide ions generated from DHX-PD-IMS-MS of unlabeled ubiqutin. (B) 2D $t_{D}(\mathrm{~m} / \mathrm{z})$ contour-plot of DHX-PD-IMS-MS analysis of unlabeled peptides extracted from the boxed region in Figure A. (C) $2 \mathrm{D} t_{D}(\mathrm{~m} / \mathrm{z})$ contour-plot the DHX-PD-IMSMS analysis of labeled peptides over the same $t_{D}(\mathrm{~m} / \mathrm{z})$ ranges

4.3. Comparing XIDTD between deuterated and undeuterated ions. (B) identification of undeuterated ions using CID. (C) Overlays of ion isotopic distributions

4.4. (A) Location of the secondary structural elements of ubiqutin atop the respective regions of primary sequence. (B) Crystal structure of ubiquitin. (C) Histogram of peptide deuterium retention.

4.5. H-D scrambling analysis using model peptide KKDDDDDIIKIIK

4.6. Per-residue deuterium content histogram via IMS-ETD-MS for $(A)$ residues $1-15$ and (B) 25-42

4.7. $t_{D}$ distributions for the identified peptide ions presented in Table 4.1

4.8. Theoretical amide deuterium content per residue

5.1. Schematic representation on $(A)$ the on-line system interfaced to $(B)$ the modified quadrupole ion trap. (C) shows the electronic components for pulsed operation during He-CTD-MS

5.2. He-CTD-MS spectrum of [KKDDDDDIIKIIK+3H] ${ }^{3+}$ precursor ions. (B) $[K K D D D D D I I K I I K+2 H]^{2+}$ precursor ions

5.3. Left and right panels show identified deuterated $c$-ions resulting from $\mathrm{He}$ CTD-MS and ETD-MS of KKDDDDDIIKIIK after on-line DHX respectively.

5.4. Istopic distribution of charge reduced ions for deuterated and undeuterated ions using for determining $\mathrm{N}$-terminal HD-scrambling most abundant ions used for per-residue deuterium calculations are labeled. 


\section{LIST OF TABLES}

2.1. Tentative plasma metabolite assignments including those contributing the most to the variation between sample cohorts

3.1. Collision cross section, HDX levels and rate constants for all major dataset features

4.1. Reproducibility of the online DHX-PD-MS system

5.1. HD-Scrambling analysis of $[\mathrm{KKDDDDDIIKIIK+3H}]^{3+} \mathrm{c}$-ions generated from 150 ETD-MS and He-CTD-MS

\section{LIST OF SYMBOLS AND ABBREVIATIONS}

$\begin{array}{ll}V_{d} & \text { Drift velocity } \\ K & \text { mobility } \\ E & \text { Electric field } \\ L & \text { Length of drift tube } \\ t_{D} & \text { Drift time } \\ N & \text { the number density of the buffer gas } \\ m & \text { lon mass } \\ \mathrm{M} & \text { Mass of the buffer gas } \\ \mathrm{Td} & \text { Townsend }\left(1 \mathrm{Td}=10^{-16} \mathrm{~V} \cdot \mathrm{cm}^{2}\right) \\ D & \text { lon diffusion constant } \\ q & \text { elemental charge } \\ k_{B} & \text { Boltzmann distribution constant } \\ T & \text { temperature } \\ \mathrm{d} & \text { sum of the radii between the ion and neutral buffer ga } \\ z & \text { lon charge } \\ \Omega & \text { Collision cross section } \\ K_{0} & \text { Reduced mobility } \\ P & \text { Pressure } \\ r & \text { Radius of drift tube entrance aperture } \\ C & \text { Constant } \\ P\left(t_{p}\right) d t_{p} & \text { time-dependent shape of the ion packet } \\ \Phi_{2}(x, y, t) & \text { quadrupolar potential } \\ U & \text { Direct current } \\ \mathrm{V}_{\mathrm{RF}} & \text { Radio frequency voltage } \\ \Omega_{a} & \text { angular frequency of the applied RF } \\ t & \text { time } \\ A_{2} & \text { constant with respect to the order of the multipoles } \\ a_{m} & \text { Mathieu parameter (function of } \mathrm{U} \text { ) } \\ q_{m} & \text { Mathieu parameter (function of } \mathrm{V}_{\mathrm{RF}} \text { ) }\end{array}$




\begin{tabular}{|c|c|}
\hline $\begin{array}{l}\omega \\
V_{\text {eff }}(r)\end{array}$ & \\
\hline $\begin{array}{l}V_{\text {efff }}(r) \\
D_{x, y}\end{array}$ & $\begin{array}{l}\text { effective electric potential } \\
\text { depth of the potential }\end{array}$ \\
\hline$r_{d}, y$ & radial distance \\
\hline$r_{0}$ & Inner radius between quadrupoles \\
\hline $\mathrm{m} / \mathrm{z}$ & Mass-to-charge ratio \\
\hline$E_{c m}$ & Center of mass energy \\
\hline$V p-p$ & Voltage peak-to-peak \\
\hline IMS-MS & Ion mobility spectrometry mass spectrometry \\
\hline MS & Mass spectrometry \\
\hline MS/MS & Tandem mass spectrometry \\
\hline$M^{n}$ & Multistage tandem mass spectrometry \\
\hline CID & Collision induced dissociation \\
\hline HCD & High energy collision induced dissociation \\
\hline IMS-CID-MS & Parallel dissociation \\
\hline XIDTD & Extracted ion drift time distribution \\
\hline ToF & Time of Flight mass spectrometer \\
\hline ETD & Electron transfer dissociation \\
\hline ETnoD & Electron transfer without dissociation \\
\hline He-CTD & Helium cation charge transfer dissociation \\
\hline ECD & Electron capture dissociation \\
\hline UVPD & Ultra-violet photodissociation \\
\hline CSIT & Charge-sign independent trapping \\
\hline TWIM & Traveling wave ion mobility spectrometry \\
\hline HDX & Hydrogen-deuterium exchange (H-to-D exchange) \\
\hline DHX & Deuterium-hydrogen exchange (D-to-H exchange) \\
\hline ESI & Electrospray ionization \\
\hline LIT & Linear ion trap \\
\hline LTQ & Linear trap quadrupole (same meaning as LIT) \\
\hline LysoPC & Lysophosphatidylcholine \\
\hline SP & Synthetic peptide \\
\hline MP & Model peptide \\
\hline PD & Pepsin digestion \\
\hline PTM & Post translational modification \\
\hline $\operatorname{ccs}$ & Collision cross section \\
\hline $\mathrm{F} 1$ & Funnel 1 \\
\hline G1 & Gate 1 \\
\hline F2 & Funnel 2 \\
\hline G2 & Gate 2 (selection gate) \\
\hline IA & Ion activation region \\
\hline SRIG & Stacked ring ion guide \\
\hline $\mathrm{nCl}$ & Negative chemical ionization \\
\hline ACN & Acetonitrile \\
\hline $\mathrm{D}_{2} \mathrm{O}$ & Deuterium oxide \\
\hline FA & Formic acid \\
\hline $\mathrm{MeOH}$ & Methanol \\
\hline
\end{tabular}




$\begin{array}{ll}\mathrm{NH}_{4} \mathrm{OH} & \text { Ammonium hydroxide } \\ \mathrm{NMR} & \text { Nuclear magnetic resonance spectroscopy } \\ \text { LC-MS } & \text { Liquid chromatography mass spectrometry } \\ \mathrm{SCX} & \text { Strong cation exchange } \\ \mathrm{GC} & \text { Gas chromatography } \\ \mathrm{EX} 1 & \text { Exchange } 1 \text { kinetics } \\ \mathrm{EX} 2 & \text { Exchange } 2 \text { kinetics } \\ N_{b k} & \text { Backbone amide hydrogen } \\ k_{\mathrm{cl}} & \text { Rate of protein closing } \\ k_{\mathrm{op}} & \text { Rate of protein opening } \\ \mathrm{ND} & \text { Deuterated ammonia } \\ \mathrm{FAB} & \text { Fast atom bombardment } \\ \mathrm{EA} & \text { Electron affinity } \\ N R M S D & \text { Normalized root mean square of the deviation } \\ C V & \text { Coefficient of variation } \\ N_{t} & \text { Total number of drift time values }\end{array}$




\section{Introduction: Expanding the Applications of Ion Mobility Spectrometry-Mass}

Spectrometry (IMS-MS) for Complex Mixtures Separations and Protein Structural Insights.

Reprinted in part with permission from Analyst: Advances in lon Mobility-Mass Spectrometry instrumentation and Techniques for Characterizing Structural Heterogeneity. Gregory C. Donohoe, Megan M. Maurer and Stephen J. Valentine, Analyst, 2015. 140(20):p.6782-6798

\subsection{Ion Mobility Spectrometry and Linear Ion Trap Mass Spectrometry}

\section{Fundamentals}

Several new applications have attempted to expand the utility of IMS-MS by implementing a new IMS-linear ion trap (LIT) mass spectrometer. The IMS separation is used in four specific and different fashions: 1) a traditional separation device for small molecule mixtures, 2) a fragmentation cell for high-throughput, data-independent analysis that requires no spectral alignment 3;) a structural gas phase deuterium labeling cell aimed at understanding protein anion structures; and, 4) as a traditional drift cell for calculating an ion's collision cross section (CCS). These four areas are then combined with the ion trapping capabilities of the LIT to perform MS ${ }^{n}$ and ETD on driftselected ions.

In experiments reported here, the IMS-MS (Figure 1.1) consists of an ion desolvation region that incorporates a dual ion funnel/ ion gate design coupled to a LTQ Velos (Thermo Electron, San Jose, CA, USA) mass spectrometer.[1, 2] A continuous beam of ions, produced via electrospray ionization (ESI), enters into a desolvation region and become focused through an hour-glass funnel $(F 1) \cdot[3,4]$ The funnel is composed of a stacked ring ion guide (SRIG) composed for ring electrodes having 
different inner diameters. The inner diameter of each electrode changes sequentially such that the cross-sectional view would appear as an hour-glass. lons entering F1 are desolvated and emerge as a focused beam of ions before becoming trapped at an electrically biased gate (G1). lon trapping is performed with the use of both RF and DC voltages applied across the ion funnel as well as a repulsive voltage at G1. Using an attractive field applied at the G1, ions are periodically pulsed into the drift tube. The drift tube is constructed of ring electrodes separated by electricial insulators. A resistor chain is connected to each electrode, where the application of voltage produces in a potential drop across the length of the drift tube ( 1 meter) resulting in a homogenous electric field $\left(\sim 10 \mathrm{~V} \cdot \mathrm{cm}^{-1}\right)$.

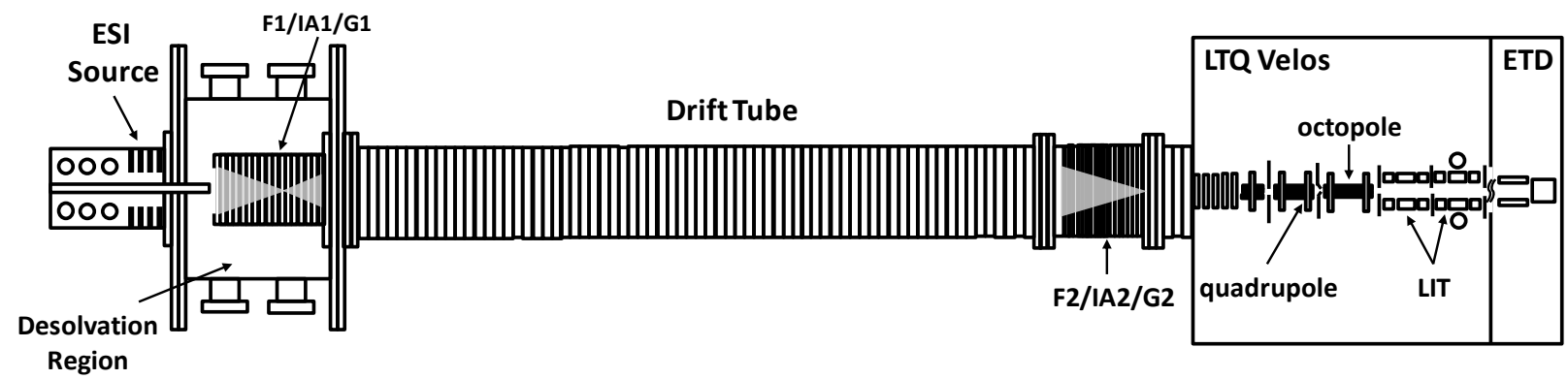

Figure 1.1. Schematic locations of the IMS-MS instrument showing the front and rear funnels $(F)$ and the locations of both gates $(\mathrm{G})$. The boxed portion of the schematic shows the LTQ Velos (ThermoScientific) mass spectrometer consisting of RF focusing devices, the linear ion trap (LIT) mass analyzer and the ETD source.

Ions that enter into the drift region undergo collisions with He gas ( 2.50 Torr at $300 \mathrm{~K})$ and separate based on differences in their three dimensional, rotationally averaged structures and overall charge. Upon reaching a selection gate (G2), ions are defined by their mobility $(K)$ as a function of ion drift velocity $\left(v_{d}\right)$ at a defined electric field strength $(E)$ according to:[5] 


$$
v_{d}=K E
$$

Here, $v_{d}$ can also be calculated by subtracting the time between a measureable ion signal at the selection gate and the introductory ion pulse. This defines the drift time $\left(t_{D}\right)$, or the transit time, for an ion population that traversed the drift tube. With this, the velocity of an ion is dependent on the pressure within the drift tube. Since $L$ and $t_{D}$ can be determined precisely determined as:

$$
v_{d}=\frac{L}{t_{D}}
$$

Combining Equation 2 with Equation 1 yields the follow relationship:

$$
K=\frac{L}{t_{D} E}
$$

Here, $K$ is inversely proportional to $E$ and proportional to $v_{d}$. From Equation 3 it can be seen the terminal velocity that the same ions attain as they tumble through the buffer gas under the influence of a homogenous electrostatic field is dictated by $K$. It is important to note that these equations reference ion motion under low-field considerations. That is, an ion's internal energy is greater than the energy gained through translational collisions with the buffer gas. Comparatively, under high-field conditions, ion internal energy would significantly increase, such that perturbations in ion shape may alter the $v_{d}$ measurement or the ions would not project their shape 
during the drift measurement. Field limits can be approximated semi-quantitatively at conditions at standard temperature:[5]

$$
\frac{E}{N}<\left(\frac{m}{m+M_{b}}\right)^{\frac{1}{2}} \frac{d^{2}}{z}
$$

where $N$ is the number density of the buffer gas at STP, $m$ and $M_{b}$ are masses of the ion and buffer gas respectively, $d$ is the sum of the radii between the ion and neutral buffer gas (angstrom) and $z$ is the charge of the ion. Equation 4, is denoted in units of Townsend $\left(1 \mathrm{Td}=10^{-16} \mathrm{~V} \cdot \mathrm{cm}^{2}\right)$, where traditional measurements, as well as the ones used in the thesis, are $\sim 1 \mathrm{Td}$. lons are generalized to move through the drift tube such that their surface area is rotationally averaged through successive and multiple collisions $\left(>10^{6}\right)$ with the buffer gas. Ion motion is also influenced by factors such as like-charge proximity, slight pressure gradients and slight differences in velocities or energies. Here, ion diffusion $(D)$ is a measure of ion spread due to Brownian motion, as expressed in the Nernst-Townsend-Einstein relation:[5]

$$
K=\frac{q D}{k_{B} T}
$$

Where, $q$ and $k_{B}$, are the elemental charge and the Boltzmann distribution constant, respectively. Notabely, $K$ is inversely related to $T$ and proportional to $q$. This equation gives insight into why ions are not discrete peaks, but broaden to some degree. Although simple, Equation 5 explains that ion motions will result in a distribution of velocities and, to some degree, the peak-shape of a drifted ion species (see below). As 
will been seen in coming chapters, the drift profile of an ion becomes a "fingerprint" and is useful for structural considerations and interrupting heterogeneity in complex mixtures.

Ultimately $K$ depends on collisions between particles. It is important to note that these collisions are influenced by $E$ and thus the forces between the ions and the buffer gas are important. Revercomb and Mason proposed this dependence in the form of a diffusion collision integral assuming ions were hard spheres and that $K$ depends on momentum transfer.[6]

$$
K=\frac{3}{16} \frac{z e}{N}\left(\frac{1}{m}+\frac{1}{M_{b}}\right)^{\frac{1}{2}}\left(\frac{2 \pi}{k_{B} T}\right)^{\frac{1}{2}} \frac{1}{\Omega}
$$

Here, $z$ and $e(q=z \cdot e)$ are the unit charge of the ion and the elemental charge of an electron respectively. The term $\Omega$ is the collision cross section (CCS) of an ion. Since the drift measurement can be performed using different parameters, it becomes instructive to report a reduced mobility $\left(K_{o}\right) . K_{o}$ allows comparisons across instrumental platforms, between laboratories or can be used once instrument performance has been optimized for a specific method. The reduced mobility is derived from scaling, calibrating or correcting to standard temperature and pressure according to:

$$
K_{o}=K\left(\frac{273.15}{T}\right)\left(\frac{P}{760}\right)
$$

Equation 6 can be rearranged to give a CCS calculation based on the ion's $t_{D}$. The equation below has been normalized to standard pressure and temperature as:[7] 


$$
\Omega=\frac{(18 \pi)^{1 / 2}}{16} \frac{z e}{\left(k_{B} T\right)^{1 / 2}}\left[\frac{1}{m}+\frac{1}{M_{b}}\right]^{1 / 2} \frac{t_{D} E}{L} \frac{760}{P} \frac{T}{273.2} \frac{1}{N}
$$

Calculating CCS values for ions allows a direct measure of a physical property. From Equation 8, the CCS calculation is dependent on the charge of the ion and the parameters $T, L$ and $P$ of the drift tube. One consideration for enhancing CCS determinations is that higher resolving power in the mobility dimension significantly enhances structural both small molecule and protein structural studies. An example is the need to adequately resolve the structural heterogeneity associated with complex mixtures such as those encountered in 'omics investigations. [8, 9] This is also true for distinguishing co-existing solution- and gas-phase structures for biomolecular ions. Resolving such fine details affords greater structural insights and more accurate CCS determinations.

The IMS resolving power $(R)$, where $\left(R=t_{D} / \Delta t_{D}\right)$ represents the ratio of the ion's $t_{D}$ to the width of the peak at half-maximum height is described by Equation 1.9.[6]

$$
R=\left(\frac{L E z e}{16 k_{B} T \ln 2}\right)^{1 / 2}
$$

In Equation 9, $R$ is dependent on $L, E$, and $T$. Shortly after the application of IMS-MS techniques for the characterization of biomolecular ion structure, researchers began to explore the development of instrumentation that would exploit parameters in Equation 9 to achieve high-resolution measurements for biological ions.[10, 11] Maximizing $R$ by changing instrument geometry often reaches a point of diminishing 
returns resulting from unmanageable operational conditions and spatial requirements. This is largely attributed to the square-root dependence in Equation 9.

One parameter not shown in Equation 9 is the pressure of the buffer gas. It is noteworthy to mention that changing the pressure of the drift cell can support higher $E$ values. The limit, as given by Paschen's Curve for various gases, defines the maximum voltage a gas can maintain at a pressure and distance before gaseous breakdown occurs. For high-resolution IMS, $300 \mathrm{~V} \cdot \mathrm{cm}^{-1}$ is possible.[12] As stated above, as long as the drift tube is maintained under low-field conditions $(E / N<3 T d)$, the above equations are applicable for characterizing ion structures and heterogeneity.

The IMS separation is based on an ion's size-to-charge ratio. lons exhibiting larger mobilities reach the detector before ions that have smaller mobilities. Often in protein structural studies, ions with similar shapes can exist over several charge states. These structures can be resolved since $K$ is proportional on the charge of an ion (Equation 6). This charge dependence allows similar structures to be resolved if ion charge is different. However in the case of isomeric structures, where $K$ is not dominated by charge, structural resolution becomes harder to predict. For a single isomer, the theoretical shape of the ion packet exiting the drift tube aperture is determined by the ion flux[13] according to:

$$
\Phi(t)=\int \frac{C}{(D t)^{\frac{1}{2}}}\left(v_{d}+\frac{L}{t}\right)\left[1-\exp \left(\frac{-r^{2}}{4 D t}\right)\right] \exp \left[\frac{-\left(L-v_{d} t\right)^{2}}{4 D t}\right] P\left(t_{p}\right) d t_{p}
$$

where $r$ is the radius of the drift tube's entrance aperture, $C$ is a constant and $P\left(t_{p}\right) d t_{p}$ is the time-dependent shape of the ion packet that is pulsed into the drift tube. Equation 
1.10 only describes a single ion conformation contributing to the overall peak shape. It is important to note that the calculated peak shape may be broader if an ion contains more than one structure or interconverts between ion structures on the time scale of the measurement. Often the former, is most instructive in observing both solution and gas phase structures without the presence of bulk solution.

\subsection{Fundamentals of Mass Spectrometry (MS) - Linear Ion Traps}

lons that traverse the drift tube reach a selection gate and pass into a second electrodynamic funnel (F2) used to radially focus the ion cloud. $[3,4,14]$ The ions subsequently exit the drift tube through a conductance-limiting aperture and are focused in the IMS-MS interface region by quadrupole and octopole RF guides before entering into the higher pressure linear ion trap followed by subsequent mass analysis in the lower pressure-linear ion trap.

In linear ion traps, ions are confined two-dimensionally (2D) by an RF field for radial confinement and DC stopping potentials applied to end electrodes for axial confinement.[15] The motion of ions in a 2D multipole field can be expressed by Newton's Laws of motion according to:

$$
F=m \frac{d v}{d t}
$$

where $F$ is the force on the ion $v$ and $t$ are the velocity of the ion and time respectively. The $\mathrm{F}$ experienced by an ion in a quadrupole potential is[15]

$$
F=-z e \nabla \Phi_{2(x, y, t)}
$$


where $\Phi_{2}(x, y, t)$ is the quadrupolar potential if applied from alternate electrodes to ground according to:[15]

$$
\Phi_{2(x, y, t)}=\frac{\left(x^{2}-y^{2}\right)}{r_{o}^{2}}\left(U-V_{R F} \cos \Omega_{a} t\right)
$$

In Equation 13, $U$ and $V$ are the applications of DC and RF potentials applied to symmetric rods, respectively. The term $\Omega_{a}$ is the angular frequency of the applied RF. For linear ion traps in a quadrupolar geometry, 2 pairs of parabolic rods are positioned adjacently one to another. This creates a well a defined inner radius $r_{o}$ associated with ion trapping. In quadrupole trapping devices, ion acceleration in the $x$ and $y$ dimensions are not coupled and ion motion in these directions are orthogonal to the central axis[15] according to:

$$
\begin{aligned}
& m \frac{d^{2} x}{d t^{2}}=-z e A_{2} \frac{\partial \Phi_{2(x, y, t)}}{\partial x} \\
& m \frac{d^{2} y}{d t^{2}}=-z e A_{2} \frac{\partial \Phi_{2(x, y, t)}}{\partial y}
\end{aligned}
$$

here $A_{2}$ is constant with respect to the order of the multipoles. These equations show that ion motion in the $x$ direction does not depend on that of the $y$ direction. This relationship is at the basis for why most $2 \mathrm{D}$ trapping devices are quadrupolar, as ion oscillation can be more easily manipulated.

In other arrangements, such as hexopoles or octopoles, $x$ and $y$ motions are coupled. 
The motion of ions in a quadrupole field as described by the dimensionless stability parameters $a$ and $q$ are:[16]

$$
\begin{aligned}
& a_{x}=-a_{y}=\frac{8 e U}{m \Omega_{a}^{2} r_{o}^{2}} \\
& q_{x}=-q_{y}=\frac{4 e V_{R F}}{m \Omega_{a}^{r} r_{o}^{2}}
\end{aligned}
$$

in Equation 1.15, term $m$ is the mass of the ion and is inversely related to both $a$ and $q$. The equations of motion in Equation 13 can be expressed as the Mathieu equations[15]

$$
\begin{aligned}
& \frac{d^{2} x}{d \xi^{2}}+\left(a_{x}-2 q_{m x} \cos 2 \xi\right) x=0 \\
& \frac{d^{2} y}{d \xi^{2}}+\left(a_{y}-2 q_{m y} \cos 2 \xi\right) y=0
\end{aligned}
$$

where $a_{m}$ and $q_{m}$ are Mathieu parameters and $\xi=\Omega_{a} t / 2$. These equations give the stability of an ion whose motion is defined as either stable or unstable within the confines of the trapping instrument. Ion trapping can be visualized by a parabolic potential well that oscillates the ions at frequency $(\omega):[15]$

$$
\omega=(2 n+\beta) \frac{\Omega_{a}}{2} ; 0 \leq \beta \leq 1 \text { and } n=0, \pm 1, \pm 2, \ldots
$$

the term $\beta$ is dependent on both $a_{m}$ and $q_{m}$ parameters. When $a_{m}$ is 0 and $q_{m}$ approaches $0, \beta$ can be approximated: 


$$
\beta \approx \frac{q_{m}}{\sqrt{2}}
$$

when $q_{m}$ is small and $n=0$, ion motion becomes harmonic with $\omega$ and can be expressed as follows:[15]

$$
\omega \approx \frac{q_{m} \Omega_{a}}{2 \sqrt{2}}
$$

Under these conditions the stability region of the ion trap becomes defined and ion confinement behaves as a function of the effective electric potential $V_{\text {eff }}(r)$ as defined by:[15]

$$
V_{e f f}(r)=D_{x, y\left(\frac{r_{d}}{r_{o}}\right)^{2}}
$$

where $r_{d}$ is the radial distance and $D_{x, y}$ is the depth of the potential well that traps the ions within a defined oscillation.[15]

$$
D_{x, y}=\frac{q_{m} V_{R F}}{4}
$$

At the center of this well ions are trapped in a specific oscillation across the $\mathrm{x}$ and y directions and are harmonic with the periodicity of the potential well. By scanning the effective potential of the trap, ions are preferentially destabilized and ejected from the 
trap and detected. In this manner, ions become characterized by their $m / z$ ratio that is dependent on the stability parameters $a$ and $q$.

Ion traps are well suited for 'omics analysis, offering high efficiency ion trapping and the ability to couple easily with either front-end techniques such as LC or GC, or for proton transfer reactions[17], ultraviolet photodissoication[18] and chemical ionization sources for ETD reactions[19] (typically interfaced at the rear of the instrument). As standalone instruments, they are considered proteomics-grade mass analyzers that operate at relatively high pressure $\left(\sim 10^{-5}\right.$ torr $)$. This makes them ideal candidates for interfacing to low pressure IMS instruments (1 -4 torr) and eliminates the need for multiple conductance limiting apertures for differential pumping stages. Ion traps also have the advantage of isolating and confining specific $\mathrm{m} / \mathrm{z}$ ratios for MS/MS or $\mathrm{MS}^{\mathrm{n}}$ analyses. Isolation is also useful for reducing space charge effects caused from ion trapping. In some cases, sensitivity can even be increased since the ejection of other ions does not destabilize the oscillation of selected ions.

In isolation experiments, all ions of measurable signal are directed into the trap and confined for mass analysis. This stage is known as the MS scan, which measures the $m / z$ of the precursor ions. Mass selection can be performed by using the trapping $\mathrm{RF}$ to resonantly excite undesired ions for ejection. In this method, multiple are frequencies are scanned sequentially. For broadband waveform isolation, all frequencies are applied except for the frequency that matches the oscillation of the intended ion for isolation.[15] lons with oscillations that are resonant with all other frequencies are excited and subsequently ejected from the trap. This type of selection is particularly useful, because ion ejection is performed quickly. However, as will be 
discussed in the following sections, special attention needs to be given to ion isolation, because waveforms close to the oscillation frequency of the isolated ion can cause unwanted vibrational excitation.

\subsection{Collision Induced Dissociation (CID)}

Once an ion has been selected within a user-defined $\mathrm{m} / \mathrm{z}$ isolation window, ions are excited with an RF frequency that is resonant with the oscillation of the ion.[16] In this manner, selected ions achieve extended oscillations, but are still confined by the ion trap. The periphery of ion traps contain $\mathrm{He}(\mathrm{g})$ used for ion confinement and cooling, but this gas can also activate the ions that have been translationally excited. The total energy available for transfer of kinetic energy into internal energy is given by the center of mass energy $\left(E_{c m}\right)$ and is dependent on the mass of the ion, the mass of the buffer gas and the laboratory frame kinetic energy. lons increase their kinetic energies from the applied RF and collide with the buffer gas. A portion of the translational energy is transferred and redistributed statistically throughout all internal modes of motions (e.g. vibrational and rational).[20] As described by the Rice-Ramsperger-Kassel-Marcus (RRKM) theory, the rate of dissociation is slow compared to the redistribution of energy. Unimolecular dissociation follows at a higher rate once the ion has achieved sufficient internal energy. Fragmentation in ion trapping MS results from the gradual heating of precursor ions that produce fragment ions and is termed tandem-in-time CID.

\subsubsection{CID of Peptides and Proteins: $b$ - and $y$-ions}

One of the greatest utilities of CID has been found in proteomics analysis for sequencing peptides and proteins. Wysocki and coworker suggest that the CID of peptides promotes the mobilization of protons for cationic ions.[21] In the mobile proton 
model, protons are transferred intramolecularly from basic and acidic residues to heteroatoms found along the backbone.[21] Proton transfer from basic residues is thought to occur at the carbonyl that facilitates nucleophilic attack of the electropositive carbon from another carbonyl. This results in a protonated oxazolone $b$-ion and the corresponding $y$-ion fragments. It is noteworthy to mention that fragmentation appears enhanced at aspartic acid residues, presumably due to solvation effects of the backbone carbonyl by the carbocylic acid. In general the $\mathrm{N}-\mathrm{CO}$ bond is dissociated resulting in $b$-and $y$-ions that are sequenced from the $N$-terminal and $C$-terminal ends respectively.[22] The use of such information can be used in de novo sequencing methods for protein primary sequence identification.[23] Newer techniques allow insilico methods, such as Sequest[24] and Mascot, to be employed for bottom-up proteomic sequencing.[25] This is particularly useful since 1000s of peptides are fragmented over the course of a chromatographic separation.

\subsubsection{CID of Small Molecules: Understanding Fragmentation}

Dissociation of complex organic molecules can be inherently more difficult than sequencing peptides. Small molecules span a diverse array of structures, classes and convalent bonding arrangements (e.g. alkene). A molecule that has obtained sufficient energy for dissociation must be reconstructed according to valence rules.[20] Structural MS of small molecules depends on the internal energy of the molecular ion and the time scale $\left(10^{-6} \mathrm{~s}\right)$ of observation. In CID processes, fragmentation should be performed quickly. If the rate of dissociation is slow, ions may fragment in transit to the detector and are observerable as metastable ions. Fragment ions of complex small molecules are complicated by competing fragmentation pathways that are dependent on the 
achieved internal energy of the precursor ion. That is, high energy CID (above $100 \mathrm{eV}$ ) can yield different spectra than low energy CID.

\subsection{IMS-MS Metabolomics and Small Molecule Studies}

Shortly after the first protein ion collision cross section measurements, IMS-MS was demonstrated as a means for separating ions within complex mixtures.[26, 27] Here, the mobility measurement was shown to distinguish isobaric ions of similar type based on differences in CCS as well as overall ion charge. Early on it was recognized that, for peptides, factors such as intramolecular interactions, side-chain packing, and overall side-chain length could affect the overall ion CCS leading to efforts aimed at predicting cross sections based on primary sequence.[28] McLean and coworkers demonstrated that many classes of small molecules could be distinguished using IMSMS techniques.[29] These early efforts laid the ground work for recent developments in small-molecule ion structure characterization/utilization using IMS-MS techniques.

Recent experiments conducted on an IMS-MS instrument that utilizes a linear, high-resolution drift tube, have yielded the most extensive database to date of CCS valuesfor small biomolecules including quaternary ammonium salts, lipids, peptides and carbohydrates.[30] The study demonstrates the potential for observing low-abundance species in the presence of higher-abundance isobaric species including isomers using IMS-MS analysis. Paglia and coworkers have demonstrated the utility of incorporating such mobility information into comparative metabolomics workflows and describe a searchable CCS database for ion identification.[31] Hill and coworkers have utilized high-resolution mobility separations to identify a new dopamine isomer obtained from striatal metabolomic extracts from genetically modified rats.[32] Similarly, experiments 
have shown that mobility measurements can be used with other analytical information (LC retention time and precursor and fragment ion masses) to distinguish isomeric species in complex mixtures obtained from natural products [33] as well as to identify potential biomarkers in comparative metabolomics analyses[34].

\subsection{An Overview of the Metabolome and Traditional Techniques}

Recent research has shown that the analysis of the plasma metabolome provides insight into fundamental metabolic processes, functions, and biomarkers associated with age, disease, and exposure to environmental influences.[35-40] Studies have revealed changes in metabolite profiles that are associated with cardiovascular disease, cancer and neurological disorders. [32, 40-44] Of particular interest, is the fact that metabolites act as signaling entities that influence cellular activity and biofunctions that include changes in protein profiles and gene expression.[37, 45] Because metabolite signaling is often involved in the initial steps of biological activity, characterization of the metabolome may serve as an important discovery tool for determining biopathways associated with transitions from healthy to disease states.[46] With this in mind, the ability to profile and identify metabolites using analytical techniques offers an opportunity for indirectly observing cellular changes associated with disease onset and progression.

According to the Human Metabolome Database, human plasma contains approximately 42,000 metabolites that mainly encompass organic acids, carbohydrates, lipids and peptides.[47, 48] Because the matrices that contain metabolites are extremely complex, analysis of the metabolome has traditionally required the use of extraction and separation techniques. Currently, most untargeted metabolomic analyses are performed 
using liquid chromatography mass spectrometry (LC-MS) because the technique routinely enables high detection numbers.[35, 49] A particular limitation of LC-MS is the data-dependent acquisition most commonly employed. Here, the mass analyzer selects the 10 most abundant ions per defined elution window. These mass-selected precursor ions are then fragmented, generating retention time information, a precursor ion $\mathrm{m} / \mathrm{z}$ measurement and fragment ion $\mathrm{m} / \mathrm{z}$ ratios. While this combination is a powerful technique, data dependent acquisition limits the amount of ions that are selected for MS/MS analysis, leading to under sampling of often important ions. While some methods employ data-independent LC-MS acquisition, it is noted that these techniques require multiple runs of the same sample (as to generate an exclusion list) that greatly increases analysis and data processing times.

One limitation regarding metabolomic analysis employing LC-MS is the time required for adequate ion identification. Even with mass spectrometers capable of high mass accuracy determinations, $\mathrm{m} / \mathrm{z}$ database matching can yield numerous isobars associated with a single spectral feature. Added to this difficulty is the identification of isomeric species that may only differ in structural arrangement (e.g., alkene positioning). Tandem mass spectrometry (MS/MS) has proven an indispensible tool for the elucidation of compound structure and thus identification.[50] As explained above, often, MS/MS is not sufficient to identify compounds of interest requiring the use of $M^{n}$.[51,52] It is noted that $M^{n}$ is difficult to perform on a chromatographic time scale, because these operations require $\sim 50 \mathrm{~ms} / \mathrm{MS}$ scan to complete.

One advantage of the dual gate IMS design is the ability to filter ions of select mobilities. Since the timing of the selection gate is delayed relative to the introductory 
pulse, a well-defined distribution of mobitiles can be transferred into the LIT. This not only reduces spectral congestion, but also concentrates ion signal prior to $\mathrm{MS}^{n}$. Because MS ion trapping can be performed up to $1000 \mathrm{~ms},>50$ IMS-pulses, containing mobility-selected ions, can be accumulated during the precursor MS scan. This is advantageous for increasing the signal of low-intensity ions that may be missed by LCMS methodologies. The combination of the mobility filtering and accumulation can be followed by MS/MS, or if signal is sufficient, $\mathrm{MS}^{n}$. As will be discussed in Chapter 2, IMS-MS ${ }^{n}$ was performed on metabolite ions and allowed for high confidence identifications.

\subsubsection{Parallel Dissociation Combined with IMS-MS for Data Independent Analysis of}

\section{Small Molecules}

The ability to rapidly profile complex mixtures using $M S / M S$ and $M^{n}$ techniques is particularly useful for identifying high numbers of molecular ions. Here, parallel dissociation stands at the forefront of high throughput methodologies. Other techniques known as data-independent or SWATH[53, 54] (AB Sciex), identify E (Waters) and all ion fragmentation (Thermo) acquisition use the mass spectrometer to fragment all observable precursor ions at each chromatographic elution window. In this manner the fragment ions are matched back to precursor ions containing similar elution profiles or retention times. However, this method is complicated by run-to-run variability caused by differences during the LC separation (e.g. mobile phase composition, pressure fluctuations, temperature, etc.). In these cases even slight changes in chromotrographic trace requires sophisticated algorithms for spectral alignment. This is further 
complicated when attempting to align fragment ions back to precursor ions based on elution profiles.

Parallel dissociation within a drift tube, (IMS-CID-MS, IMS $\left.{ }^{n}-M S\right)$ has been previously described and demonstrated for 'omics applications using ToF[55, 56] and ion trapping mass analyzers.[57] As discussed, nested experiments record ion drift times as they are pulsed into the ToF flight tube. It is noteworthy to mention that IMSCID-ToF instruments perform parallel dissociation at the end of the mobility separation. While the technique has been successful, performing CID directly after the mobility separation can perturb the drift profile due to differences in transit times of fragment and precursor ions.

For the studies reported here, a dual gate IMS-MS is employed and is well suited for IMS-CID-MS techniques. One difference is that the pulse delay applied to the selection gate allows ion filtering, where only a specific mobility is selected. Once selected, the ions contain drift information prior to mass analysis. Fragmentation is performed at the second ion activation region (IA2 in Figure 1.1) after ion selection has been made. Ion acitivation is achieved by increasing the voltage difference across two adjacent electrodes. This results in energizing collisions with the buffer gas leading to fragmentation. In this manner the region immediately following the drift tube is used as a collision cell. Since the precursor ions were previously selected, they transfer drift information to the fragment ions. All ions are then transferred to the mass analyzer for $\mathrm{m} / \mathrm{z}$ analysis. Although this process increases the complexity of the sample, fragment ions can be matched back to their respective precursor ions based on extracted ion drift time distributions (XIDTDs). Coupling XIDTDs with the high reproducibility of the IMS 
separation, requires no dataset alignment in comparative analyses and enhances spectral deconvolution. As will be described in Chapter 2, fragment XIDTDs that have similar spectral features are capable of being matched back to their respective precursor ion drift traces. This high level of specificity is required for such high throughput data independent workflows.

\subsection{Electron Transfer Dissociation (ETD) of Peptides and Proteins}

Perhaps the first demonstration of radically fragmenting peptides and proteins with low energy electrons was performed by McLafferty and coworkers in the form of electron capture dissociation (ECD).[58] These experiments used a Fourier transform ion cyclotron resonance mass spectrometer (FT-ICR-MS) equipped with an emission cathode and a grid. A voltage applied across the cathode resulted in the emission of thermal electrons $(\sim 1 \mathrm{eV})$ that could be trapped in the ICR cell and reacted with peptide cations in the gas phase. Capture of the electron by the peptide cations resulted in the dissociation of the peptide cations into fragment components. Unlike CID, that produces $b$ and $y$ ions through vibrational activation, ECD causes radical-directed dissociation of the $\mathrm{N}-\mathrm{C}_{\alpha}$ bond and produces even electron $c$ - and odd electron $z$-ions.

Such ECD experiments let to the advent of ETD for peptide/protein fragmentation shortly later. Although ECD must be performed within the low pressure environment of an ICR cell ( $10^{-10}$ torr), ETD can be performed in higher pressure mass analyzers, such as the LIT. The process of fragmentation with ETD is much different than that reported for ECD; however the two techniques both produce $c$ - and $z$-ions via the same mechanism. Electron transfer dissociation was first reported by Hunt and coworkers using a LIT interfaced to a chemical ionization $(\mathrm{Cl})$ tower at the rear of an LIT 
instrument.[19] This tower contains a heated cell for the sublimation of fluoranthene that is swept into the negative $\mathrm{Cl}$ chamber by a flow of $\mathrm{N}_{2}(\mathrm{~g})$. The excess flow of $\mathrm{N}_{2}(\mathrm{~g})$ into the $\mathrm{Cl}$ chamber produces free electrons that are captured by sublimated fluroanthene. In turn, the fluranthene anions become charged, and their trajectories can be manipulated in the gas phase. Reagent anions are transfered from the ionization chamber to the rear of the LIT via ion guides. It is important to note that the commercial LIT, used in the studies for this thesis, is segmented into high and low pressure regions and is highly suitable for ETD. Here, fluoranthene radicals transferred into the low pressure trap are subsequently isolated, purified and accumulated prior to ion-ion reactions. At the same time, the high pressure trap is accumulating peptide/protein cations. lons of opposite charge are held in oscillation within their respective trapping regions. Once enough fluoranthene radicals have been generated, ETD is performed by adjusting the RF potential applied to the end lenses or the matching rod sets. This provides charge-sign independent trapping (CSIT), which axially confines both positive and negative ions simultaneously. The ETD reaction generally proceeds for $\sim 100 \mathrm{~ms}$, after which, the LIT scans and detects fragment ions.

\subsubsection{ETD Mechanism: $c-$ and $z-$ ions}

The collision between anions and cations causes the transfer of an electron from the fluoranthene radical to the peptide. Electron transfer is thought to occur at a protonated basic side chain such as lysine, arginine or histidine, forming a hypervalent radical species.[58] The electron occupies an excited Rydberg state that upon relaxation transfers a hydrogen from the basic sidechain to the backbone carbonyl.[59] This process produces a carbon radical aminoketyl intermediate. This is followed by 
radical rearrangement and cleavage, where half of the electron density of the $\mathrm{N}-\mathrm{C}_{\alpha}$ bond, combines with the carbon radical and the other half is retained on the $\mathrm{C}_{\alpha}$ carbon. The rearrangement is radically driven and produces $-c$ and $z$ - fragment ions. Unlike CID, ETD proceeds through a nonergodic mechanism and dissociation occurs before the redistribution of energy $\left(\sim 10^{-12} \mathrm{~s}\right)$. This mechanism is an important consideration, because unlike CID, ETD can preserve post translational modifications (e.g. phosphorylation and glycosylation) and is necessary for per-residue hydrogen deuterium exchange (HDX) measurements. Both subjects are discussed below. 1.6.2. IMS - ETD - MS Techniques

While biomolecular applications of CID combined with IMS occurred almost concurrently, IMS coupled with ETD is a relatively newer concept. Experiments by de Pauw and coworkers use ETD of cysteine containing peptides prior to the mobility separation.[60] Peptide ions involved with charge reduction were shown to have more extended conformations compared to the same ion that was not subjected to ETD reagent. Post mobility the reduced ions were collisionally activated and the site of disulfide bonding was elucidated. Other early studies used ETD followed by traveling wave ion mobility (TWIM) to locate cis-platin interactions with methionine containing peptides.[61] After ETD, the fragments were mobility separated and easily identified based on differences in arrival time distributions. Other studies have used the ETDTWIM instrument for other peptide and protein workflows.[61-63] While these works are interesting, it is also noted that the ETD cell is located before the mobility region, $[64,65]$ meaning that the mobility separation is not utilized to distinguish precursor ion conformers. To the best of my knowledge, ETD on a mobility separated ion has only 
been performed on a field asymmetric ion mobility spectrometry instrument (FAIMS).[66] However FAIMS operates above the low-field limit and cannot be used to determine CCS values. With this in mind, the ability to perform seminal studies using a linear field drift tube coupled with ETD-MS expands the applications of 'Omics analyses into new frontiers.

\subsection{Phosphoproteomics and MS Techniques}

Protein phosphorylation is a reversible covalent post-translational modification occurring at tyrosine, serine, and threionine residues. $[67,68]$ This modification is added or removed by kinases and phosphtases, respectively, making phosphorylation a temporally dynamic process. Phsophorylation is the most common PTM for eukaryotic organisms and implies a specific importance in regulating protein activity, cellular function and signaling pathways.[68,69] A particular challenge of phosphoproteomics is first detecting and then identifying the site of phosphorylation. Simply measuring protein abundance does not correlate with the stiocheometric amount of phosphorylation. That is, residues that are modified are often low abundance, making enrichment and multidimensional separations necessary.

Mass spectrometry has become a powerful tool for identifying and determining the site of phosphorylation. Identification of the phosphate moiety is complicated due to the phosphodisester bond. That is, under CID conditions, the bond is labile and energizing collisions cause the neutral loss of the phosphate moiety.[70, 71] This can be used advantageously for neutral loss scanning approaches employing triple quadrupole $(\mathrm{QqQ})$ mass filters. Here the first quarupole (Q1) scans precursor ions which undergo CID in the middle quadruple (q). The last quadrupole (Q2) scans for the neutral loss of 
$\mathrm{H}_{3} \mathrm{PO}_{4}$. An important consideration of this technique is the observable charge state of the precursor ion, as this influences the fragmentation energy and accessible dissociation pathways resulting in observable fragments. Collision induced dissociation of the precursor ion containing the phosphate moiety can induce several neutral losses. These include $\mathrm{m} / \mathrm{z} 79\left(\mathrm{PO}_{3}{ }^{-}\right), 80\left(\mathrm{HPO}_{3}\right) 98\left(\mathrm{H}_{3} \mathrm{PO}_{4} ; \mathrm{HPO}_{3}+\mathrm{H}_{2} \mathrm{O}\right), 63\left(\mathrm{PO}_{2}{ }^{-}\right)$and 97 $\left(\mathrm{H}_{2} \mathrm{PO}_{4}\right)$. Because the backbone amide bond dissociation energy is $\sim 40 \mathrm{kcal} / \mathrm{mol}$ and the phosphodiester linkage is $\sim 20 \mathrm{kcal} / \mathrm{mol}$, the collision energy can be tuned such that fragmentation does not produce a significant amount of $b$ - or $y$-ions. Here, neutral loss fragment ions can then be scanned by Q2 to detect the presence of phosphorylation.[71] While this technique excels at detecting the presence of phosphorylation, locating the site of phosphorylation requires the use of CID-MS ${ }^{n}$ or ETD-MS.

More recent experiments involve phosphopeptide enrichment using $\mathrm{TiO}_{2}$ affinity columns, followed by reverse-phase LC-MS. This technique is capable of observing > 36,000 phosphopetides using a bottom-up approach from a tryptic digest of HeLA cells.[72] Other techniques use strong cation exchange (SCX),[73] and antibody enrichment strategies.[74] With the advent of high resolution ion trap mass analyzers (such as the Orbitrap), targeted MS/MS techniques coupled with in-silico database searching can identify the site of phosphorylation using CID or high-energy collision induced dissociation (HCD).[75] The use of powerful software for necessary in predicting and identifying the neutral loss of the phosphate moiety. This information is then related back to the specific residue that contained the modification. 
Other fragmentation methods such as ETD have also been used to identify phsophorylation. Compared to CID, ETD can result in extensive backbone cleavage without losing the phosphate moiety.[71, 76, 77] As discussed above, ETD is a nonergodic mechanism that proceeds without excessive virbational heating. This application makes sequencing much easier than CID, because the spectra are not dominated by neutral loss events. One limitation regarding ETD is that precursor ions must have high charge densities ( $\geq+2$ ) for complete fragmentation. If this requirement is not met, the ion-ion reaction often proceeds through electron transfer without dissociation (ETnoD).[78, 79] Some studies have combined the use of CID with MSETD. Here, the CID (in-source dissociation) event results in the neutral loss of the phosphate moiety. The intact neutral loss fragment is then transferred to MS for $\mathrm{MS}^{\mathrm{n}}$ or MS-ETD. These experiments are referred to as CID triggered MS/MS. In this manner the presence and identify of the phosphopeptide is obtained. As described above, one limitation is identifying the neutral loss event since many combinations of phosphate dissociation are possible.

\subsubsection{Coupling IMS-CID-ETD-MS for Determining the Presence and Location of} Phosphorylation for Peptides Within Complex Mixtures.

Neutral loss scanning and ETD-MS techniques have their limitations and merits. The neutral loss scanning approach can easily identify the presence of phosphorylation events within complex mixtures, but can be limited by its ability to identify modified residues. On the other hand, ETD stands as a capable approach of easily identifying residues that have been post translationally modified. It therefore becomes desirable to create methods that can separate complex mixtures quickly, while detecting 
phosphorylation and identifying modified residues. Ion mobility has been used in the past to observe structural aspects of phosphorylation on protein and peptide structure. Additionally, parallel dissociation techniques combined with IMS-MS have been used for observing differences in fragmentation behavior of phosphorylated peptides. In Chapter 2 of this thesis, the capability to rapidly identify phosphorylated peptides in tryptic digests that would be encountered in post-translational modification (PTM) experiments is demonstrated. For these studies, IMS-CID methods show that targets for PTM analysis can rapidly be ascertainedand subsequently subjected to ETD to identify phosphorylation sites. To some degree, the approach is similar to proteomics techniques that used triggered CID followed by ETD-MS. A difference is that XIDTD helps to confirm and isolate ions for subsequent tandem MS experiments.

\subsection{Solution Hydrogen Deuterium Exchange: Fundamentals}

Protein hydrogen deuterium exchange-mass spectrometry (HDX-MS) is an isotopic labeling strategy involving the exchange of heteroatom hydrogens with deuterium over a defined period of time.[80-82] The main focus of HDX-MS methodology is to elucidate protein structural regions[83, 84], folding dynamics[80, 8591] and protein interactions[92-96] via mass shifts caused by the incorporation of deuterium after the exchange event. Most commonly, these exchange reactions involve the incubation of protein within a buffered solution containing deuterium oxide. The overall exchange mechanism can be described by:[80]

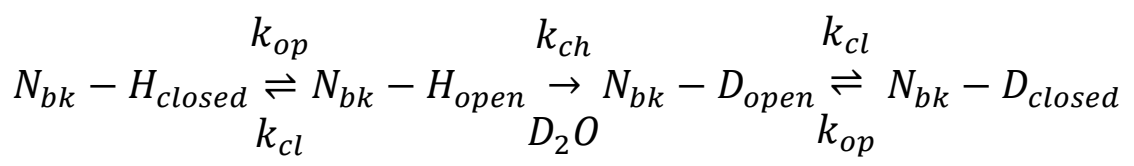


where $N_{b k}$ is a backbone amide along the proteins primary sequence. Due to random vibrations and motions of the protein, temporary conformations allow the protein to open at a rate $k_{\mathrm{op}}$ and close back to the initial conformation at a rate of $k_{\mathrm{cl}}$. If $k_{\mathrm{op}}$ is such that solvent $\mathrm{D}_{2} \mathrm{O}$ is capable of interacting, then $\mathrm{N}-\mathrm{H} \rightarrow \mathrm{N}-\mathrm{D}$ exchange occurs at a rate $k_{c h}$. The term $k_{c h}$ is the intrinsic rate constant and is determined by the flanking side chains, as well as the temperature and $\mathrm{pD}$ of the solution. If all rates are favorable, then deuterium is incorporated into the protein before the region closes and is no longer solvent accessible.

The exchange mechanism is base catalyzed, and occurs with hydroxide abstracting a hydrogen from a solvent-accessible hetero-atomic site.[80] Deutration follows and is driven by the free amount of $\mathrm{D}^{+}$in the solution. It is easy to see that $k_{\mathrm{op}}$, $k_{c l}$ and $k_{c h}$ can give several combinations of exchange rates. In general exchange has been divided into two separate regimes referred to as EX1 and EX2. In EX1, $k_{c h}>>k_{c 1}$ and suggests that HDX occurs during the initial opening transition.[97, 98] Comparatively, under the EX2 regime, $k_{c h} \ll<k_{c l}$ and implies that exchange occurs over many opening and closing cycles before $\mathrm{N}-\mathrm{H} \rightarrow \mathrm{N}-\mathrm{D}$ exchange occurs.[99] EX2 suggests that bonding networks found in secondary, tertiary and quaternary scaffolds are less frequented by intermolecular interactions with the aqueous solution, thereby resulting in slower exchange kinetics for structured regions. As is often the case, most systems exhibit EX2 kinetics.

While the above equations reference the exchange from $\mathrm{H} \rightarrow \mathrm{D}$, it is noted that in the coming chapters $\mathrm{D} \rightarrow \mathrm{H}$ exchange was used. This is commonly called deuterium hydrogen exchange or backexchange.[80] Here, the protein is first incubated in $\mathrm{D}_{2} \mathrm{O}$ to 
a level of $\sim 100 \%$ deuterium incorporation. The rate constants and mechanism of action are the same as described above; however, the deuterated protein is exposed to a hydrogen source. In this manner the representation of secondary structure is indicated by regions that will retain deuterium. Comparatively, unstructured regions will backexchange to hydrogen. In this manner, mass shifts directly suggest areas of secondary structure.

1.8.1. Bottom - up Hydrogen Deuterium Exchange - Mass Spectrometry (HDX - MS)

Most common HDX-MS workflows employ a bottom-up technique. Here, the protein is placed into a $D_{2} \mathrm{O}$ rich buffer and exchange occurs over a defined period of time. Kinetic studies can be performed to determine both EX1 and EX2 regimes based on exchange profiles after varying the incubation time. The exchange also gives an idea of the relative protection of a particular area, since unstructured areas will exchange at higher rates (up to some maximum) than structured regions. Exchange events are quenched at low $\mathrm{pH}(\sim 2.5)$ and temperatures approaching $0{ }^{\circ} \mathrm{C}$. Under these conditions the protein is digested with pepsin, because the enzyme displays high activity under quench conditions. These conditions are used to limit backexchange, which is a major concern for bottom-up approaches. Because the protein has been digested, the native structure is lost and any further exchange may change the true deuterium content level of the peptide sequence. Many HDX-MS experiments use LC systems consisting of an immobilized pepsin column for online digestion followed by trapping and reverse-phase separation of peptic fragments that are subsequently mass analyzed.[100,101] Using this "bottom-up" approach, deuterium incorporation at backbone amide locations can be evaluated on a per-peptide basis. 
Despite much success,[86, 102, 103] bottom-up HDX-MS is limited by spatial resolution that is dependent upon the number of overlapping peptide fragments. That is, per-residue analysis of deuterium incorporation must contain two peptides that differ by one residue in length. Since many proteolytic peptides increase the overall overlap, and thus spatial resolution, multiple catalytic enzymes have been used; however, this creates a highly complex dataset.[104, 105]Due to the incorporation of deuterium, mass measurements can be made and structural insights can be ascertained based on uptake profiles across a particular identified sequence.

\subsubsection{Top-down HDX-MS}

Top-down approaches using non-ergodic fragmentation techniques such as electron capture dissociation (ECD) and electron transfer dissociation (ETD) have shown improved spatial resolution without deuterium scrambling (see below).[106-109] These workflows are similar to that described above. That is, the protein is incubated in $\mathrm{D}_{2} \mathrm{O}$ over several periods of time. The protein is electrosprayed in positive polarity mode. Here, the low pH environment of the quench solution is highly suitable for positive ESI and mass analysis. Also noteworthy is that under these conditions the protein is denatured and higher charge states can be achieved. In turn, these high charge states are highly suitable for electron-based fragmentation methods, as the reaction cross sections are larger and ECD or ETD can proceed at higher efficiencies (greater sequence coverage). Once fragmented in the gas phase, Top down approaches can determine deuterium incorporation at a per-residue level. This is different than the bottom up approaches that observe the global view based on peptide sequence. However, larger proteins (>20 kDa)[110] and inadequate charge states 
remain problematic for electron-based fragmentation. Other groups have coupled LCMS/MS based techniques for fragmentation of deuterated proteolytic peptides, using a bottom-up workflow.[107, 111]

\subsubsection{Per- residue Deuterium Measurements}

Tandem mass spectrometry (MS/MS) presents a technique that is well suited to site-specific (per-residue) deuterium retention using both top-down[106, 107, 109] and bottom-up[112, 113] approaches. Early HDX-MS/MS studies employed collisioninduced dissociation (CID) to elucidate per-residue deuterium incorporation.[114, 115] In general, CID relies on the conversion of translational energy to internal energy via inelastic collisions of selected ions with an inert buffer gas such as helium. Through multiple collisions, the internal energy of the molecular ion increases and fragmentation occurs at the oscillator with the lowest bond dissociation energy. For proteins and peptides, CID predominantly produces $b$ - and $y$-type fragment ions.[20]

Although CID combined with HDX has shown some success, $[115,116]$ one limitation is hydrogen/deuterium (HD)-scrambling. Because CID is an ergodic process, energy is redistributed and CID is accompanied by the mobilization of protons (see above).[21] These mobile protons, found on both acidic and basic residues, are transferred throughout the molecule and participate in the fragmentation process.[21] Because proton mobilization occurs before dissociation in CID, the final location of the proton on the product ion is typically different than the initial location on the precursor ion. Mobilization is obviously problematic when using HDX-MS/MS to target structural areas because redistribution occurs as readily for mobile deuteriums as it does for mobile hydrogens. That is, structured regions expected to contain higher levels of 
deuterium will appear lower than the "true" deuterium content value. Conversely, unstructured regions are artificially enriched. In such cases, per-residue measurements provide ambiguous or erroneous data for structural information.

More recently, electron capture dissociation (ECD) and electron transfer dissociation (ETD) have been shown to fragment deuterated precursor ions without HDscrambling[108, 111, 113, 117-119]. In contrast to CID, electron-based fragmentation of biomoleuclar ions proceeds through a high energy mechanism. In ECD and ETD, the capture or transfer of a near thermal electron to the biomolecular ion results in shortlived, odd electron intermediates. Fragmentation is radical-driven and occurs rapidly before proton mobilization throughout the molecular ion. ECD and ETD primarily produces $c$-and $z$-ion series with significantly less contribution of $b$ - and $y$-ions. Although ECD and ETD can involve the transfer of a proton from a basic side chain to a c-ion,[120] such observations can be accounted for and are more predicable than proton mobilization associated with CID. Importantly, because electron excitation occurs before proton randomization, per-residue HDX studies typically proceed without the loss of the initial deuterium label.

\subsubsection{Solution HDX Coupled with IMS - ETD - MS}

Ion mobility spectrometry-mass spectrometry has emerged as a powerful tool for separating complex mixtures containing biomolecules.[26, 27, 121-126] The gas phase separation is rapid (milliseconds), affording increased peak capacity at no cost to MS analysis times.[127, 128] A particular advantage of IMS is its ability to decrease the lower-detection limit by removing highly-abundant ions from spectral regions containing interfering ions.[47, 125, 129, 130] Thus, by extracting a specific mobility distribution 
within the 2D IMS-MS dataset, a better evaluation of precursor ions that may be suppressed during ionization is obtained. It is noteworthy to mention that the IMS separation occurs in the gas phase. This eliminates the process of solution backexchange found in condensed-phase separations.

To date, few studies have explored the potential in coupling ion mobility with solution phase HDX. However, some studies have used commercial TWIM devices coupled with Time-of-flight mass analyzers for bottom-up peptide studies.[131, 132] These studies showed that that incorporation of deuterium did not change the arrival time distributions of deuterated peptides, relative to the corresponding undeuterated peptides. Also noted is the ability of the mobility measurement to separate overlapping deuterated peptides. This is particularly advantageous since deuterium incorporation broadens the isotopic distribution and results in reduced peak capacity. Moreover, isotopic overlap between neighboring ions interferes with deuterium calculations. This is problematic for accurately determining deuterium content for both overlapping species.

\subsection{Gas Phase Hydrogen Deuterium Exchange Coupled with IMS-MS}

In 2009, Engen and coworkers showed that mobility measurements could be combined with gas-phase $\mathrm{HDX}$ using $\mathrm{ND}_{3}$ as a deuterating reagent.[63] Different conformer types of ubiquitin ions could be distinguished by their unique mobilities and HDX levels. Rand and coworkers later showed that HDX could be accomplished for protein ions in a TWIM instrument with site-specific determination of the label incorporation.[62] Later the research group demonstrated different operational modes for performing gas-phase HDX measurements with IMS-MS techniques using a TWIM instrument.[133] Ashcroft and coworkers monitored changes in protein ion structure 
resulting from solution perturbations using gas-phase HDX-MS techniques.[134] The resulting data was shown to correlate to mobility information obtained from IMS-MS measurements. Valentine and coworkers demonstrated the first determination of sitespecific deuterium incorporation for mobility-selected biomolecular ion conformations using a drift tube coupled to a linear ion trap outfitted with ETD capabilities.[135] The researchers then showed that the contributions by individual amino acid residues to conformer type exchange rate could be determined.[136] Using the experimental results and a kinetics model, the authors were able to show that multiple ion conformers are likely to comprise many mobility selections.

Early theoretical studies resulted in proposed mechanisms for HDX of protonated peptide ions by a number of deuterating agents. $[137,138]$ The use of $\mathrm{ND}_{3}$ and $\mathrm{D}_{2} \mathrm{O}$ are the most common deuterating reagents for gas phase HDX employing IMS. In the studies reported here, $D_{2} \mathrm{O}$ is exclusively used for gas phase labeling experiments. For gas phase HDX using $\mathrm{D}_{2} \mathrm{O}$, a relay mechanism has been computationally determined and supported by and experimental results.[139] In this model, biomolecular ions collided with $\mathrm{D}_{2} \mathrm{O}$ vapour and form a long-lived complex. Upon complexation the free energy of the system is lowered due to hydrogen bonding. Exchange commences as a proton is shuttled from a heteroatomic charge site of the ion to $\mathrm{D}_{2} \mathrm{O}$. A second hydrogen bond results in simultaneous transfer of deuterium to a distant, less basic site or to the site of interaction. Dissociation of the complex results with no net gain or loss to the free energy of the system.

Initial IMS-HDX-MS studies reported the maximum HDX levels as well as rates of exchange for a number of cytochrome $c$ ion conformers.[140] These early studies 
demonstrated that compact cytochrome $c$ ion conformers exhibited decreased levels of HDX compared with more elongated ions at room temperature. Additionally, the overall HDX levels were compared with those reported for the same ion charge states as measured in a Fourier transform ion cyclotron resonance mass spectrometer. In general the HDX levels recorded for the mobility-resolved conformers was determined to be smaller than those recorded for ions in the FTICR. This was explained as possibly arising from the longer timescale of the latter measurement rendering the observed exchange subject to longer timescale structural fluctuations.

Although the gas-phase HDX characteristics of negatively charged oligonucleotides and small molecules including amino acids have been studied,[141146] HDX of protein and peptide anions has received considerably less attention.[147] As a consequence, no experiments describing HDX characterization of peptide and protein anions exists. Because many proteins contain a large number of acidic residues, it is useful to develop a sound understanding of the conformational information afforded by gas-phase HDX measurements for select conformers from negativelycharged protein ions. Moreover, the degree of HDX scrambling occurring for activated, negatively-charged peptide ions has recently been reported.[148] Such information begins to lay the foundation for an understanding of intramolecular deuterium migration which can further clarify the structural information obtained from HDX experiments. Finally, it should be noted that with the rapid development of novel ion fragmentation techniques,[19, 58, 149-154] the experiments described here are timely; that is, this work leads to future studies allowing the determination of residue-specific deuterium uptake for select anion conformers. 


\subsection{Helium Charge Transfer Dissociation}

Electron based fragmentation processes like ECD and ETD are dependent on the charge state of the precursor ion. Given that many bottom up workflows produce highly digested peptides of low charge state, per-residue measurements without HDscrambling can therefore be difficult to obtain. Both ECD and ETD techniques can fragment peptides exhibiting charge states $\geq 2$; however, complete sequence information is often limited because ETnoD becomes the dominant product ion pathway. With this in mind, it would be highly desirable to have access to a fragmentation technique that could proceed via odd electron or radical-induced pathways for low charge state precursors without proton mobilization

Recently, a new MS/MS technique known as Helium charge transfer dissociation (He-CTD) of peptide and protein ions has been demonstrated using either helium cations[155] or cations from an air plasma.[156] Gaseous ions emitted from a fast atom bombardment (FAB) gun achieve kinetic energies sufficient to overcome the Coulomibic barrier with biomolecular cations. These cationic species are directed into an ion trapping instrument, where peptide cations have been previously mass-selected. $\mathrm{He}-$ CTD product ions are seen to result from both vibrationally- and radically-driven dissociation pathways that resemble those formed from both CID and ECD/ETD and ultraviolet photodissociation (UVPD) processes.[155, 156] In other cases, ion-ion reactions result in both non-dissociative charge reduction and increase (gas-phase supercharging).[156] Of particular interest is the ability of He-CTD to produce radical fragmentation regardless of precursor ion charge state. Although the processes that influence these observations are currently difficult to pinpoint, the capability of 
fragmenting low charge state precursor ions may offer an improvement over traditional techniques (i.e. ETD).

Because of the short interaction times, He- CTD is presumed to follow vertical activation (not adiabatic), and has been shown to fragment neutral molecules with appearance potentials on the order of $30 \mathrm{eV}$.[155] He-CTD therefore activates precursor ions through electronic and vibration modes. Fragment ions $y, b, c$ and $z$ ions were identified. In chapter 5, experiments consider the $c$ ion series after He-CTD MS measurements. CTD is performed with $\mathrm{He}^{+}$cations emitted with high kinetic energy (6 $\mathrm{KeV}$ ) from the saddle field source. The main purpose of kiloectronvolt energies is to overcome the Coulombic barrier of cation/cation reactions. Perhaps because of inelastic transfer of energy during charge transfer, the activation energy obtained through $\mathrm{He}^{+}$ CTD exceeds the electron affinity of the $\mathrm{He}^{+}$cation and is on the order of $30 \mathrm{eV}$.[155] The formation of $c$ ions can result from proximal reagent $\mathrm{He}^{+}$ions abstracting an electron (EA $24.6 \mathrm{eV}$ ) and creating a hole in the precursor ions. In turn, the electron deficient ion undergoes radical fragmentation. 


\subsection{References}

1. Lee, S., et al., Extracted fragment ion mobility distributions: A new method for complex mixture analysis. International Journal of Mass Spectrometry, 2012. 309: p. 154-160.

2. Zucker, S.M., et al., An Ion Mobility/lon Trap/Photodissociation Instrument for Characterization of Ion Structure. Journal of the American Society for Mass Spectrometry, 2011. 22(9): p. 1477-1485.

3. Kelly, R.T., et al., The ion funnel: Theory, implementations, and applications. Mass Spectrometry Reviews, 2010. 29(2): p. 294-312.

4. Shaffer, S.A., et al., An ion funnel interface for improved ion focusing and sensitivity using electrospray ionization mass spectrometry. Analytical Chemistry, 1998. 70(19): p. 4111-4119.

5. Viehland, L.A. and E.A. Mason, Transport Properties of Gaseous lons over a Wide Energy Range, IV. Atomic Data and Nuclear Data Tables, 1995. 60(1): p. 37-95.

6. Revercomb, H.E. and E.A. Mason, THEORY OF PLASMA CHROMATOGRAPHY GASEOUS ELECTROPHORESIS - REVIEW. Analytical Chemistry, 1975. 47(7): p. 970-983.

7. Mason, E.A.M., E. W., Transport Properties of lons in Gases. 1988, New York: Wiley.

8. Dwivedi, P., et al., Metabolic profiling by ion mobility mass spectrometry (IMMS). Metabolomics, 2008. 4(1): p. 63-80.

9. Myung, S., et al., Development of high-sensitivity ion trap-IMS-TOF techniques: A high-throughput nano-LC/IMS/TOF-separation of the Drosophila melanogaster proteome. Abstracts of Papers of the American Chemical Society, 2003. 226: p. U112-U112.

10. Dugourd, P., et al., High-resolution ion mobility measurements. Review of Scientific Instruments, 1997. 68(2): p. 1122-1129.

11. Asbury, G.R. and H.H. Hill, Evaluation of ultrahigh resolution ion mobility spectrometry as an analytical separation device in chromatographic terms. Journal of Microcolumn Separations, 2000. 12(3): p. 172-178.

12. Tang, X., J.E. Bruce, and H.H. Hill, Design and performance of an atmospheric pressure ion mobility Fourier transform ion cyclotron resonance mass spectrometer. Rapid Communications in Mass Spectrometry, 2007. 21(7): p. 1115-1122.

13. Moseley, J.T., et al., Measurement of Transport Properties of lons in Gases; Results for $\$\{\mid m a t h r m\{K\}\}^{\wedge}\{+\} \$$ lons in $\$\{\mid$ mathrm $\{N\}\} \_\{2\} \$$. Physical Review, 1969. 178(1): p. 234-239.

14. Tang, K., et al., High-sensitivity ion mobility spectrometry/mass spectrometry using electrodynamic ion funnel interfaces. Analytical Chemistry, 2005. 77(10): p. 3330-3339.

15. Douglas, D.J., A.J. Frank, and D. Mao, Linear ion traps in mass spectrometry. Mass Spectrometry Reviews, 2005. 24(1): p. 1-29.

16. March, R.E., An Introduction to Quadrupole Ion Trap Mass Spectrometry. Journal of Mass Spectrometry, 1997. 32(4): p. 351-369. 
17. Mielke, L.H., et al., Development of a Proton-Transfer Reaction-Linear Ion Trap Mass Spectrometer for Quantitative Determination of Volatile Organic Compounds. Analytical Chemistry, 2008. 80(21): p. 8171-8177.

18. Kim, T.-Y., M.S. Thompson, and J.P. Reilly, Peptide photodissociation at $157 \mathrm{~nm}$ in a linear ion trap mass spectrometer. Rapid Communications in Mass Spectrometry, 2005. 19(12): p. 1657-1665.

19. Syka, J.E.P., et al., Peptide and protein sequence analysis by electron transfer dissociation mass spectrometry. Proceedings of the National Academy of Sciences of the United States of America, 2004. 101(26): p. 9528-9533.

20. Shukla, A.K. and J.H. Futrell, Tandem mass spectrometry: dissociation of ions by collisional activation. Journal of Mass Spectrometry, 2000. 35(9): p. 1069-1090.

21. Wysocki, V.H., et al., Mobile and localized protons: a framework for understanding peptide dissociation. Journal of Mass Spectrometry, 2000. 35(12): p. 1399-1406.

22. Roepstorff, P. and J. Fohlman, Proposal for a common nomenclature for sequence ions in mass spectra of peptides. Biomed Mass Spectrom, 1984. 11(11): p. 601.

23. Dancik, V., et al., De novo peptide sequencing via tandem mass spectrometry. J Comput Biol, 1999. 6(3-4): p. 327-42.

24. Eng, J.K., A.L. McCormack, and J.R. Yates, An approach to correlate tandem mass spectral data of peptides with amino acid sequences in a protein database. Journal of the American Society for Mass Spectrometry. 5(11): p. 976-989.

25. Boyd, R. and Á. Somogyi, The Mobile Proton Hypothesis in Fragmentation of Protonated Peptides: A Perspective. Journal of the American Society for Mass Spectrometry, 2010. 21(8): p. 1275-1278.

26. Valentine, S.J., et al., Gas-phase separations of protease digests. Journal of the American Society for Mass Spectrometry, 1998. 9(11): p. 1213-1216.

27. Gillig, K.J., et al., Coupling high-pressure MALDI with ion mobility/orthogonal time-of flight mass spectrometry. Analytical Chemistry, 2000. 72(17): p. 39653971.

28. Valentine, S.J., et al., Intrinsic amino acid size parameters from a series of 113 lysine-terminated tryptic digest peptide ions. Journal of Physical Chemistry B, 1999. 103(8): p. 1203-1207.

29. Fenn, L.S. and J.A. McLean, Biomolecular structural separations by ion mobilitymass spectrometry. Analytical and Bioanalytical Chemistry, 2008. 391(3): p. 905909.

30. May, J.C., et al., Conformational Ordering of Biomolecules in the Gas Phase: Nitrogen Collision Cross Sections Measured on a Prototype High Resolution Drift Tube Ion Mobility-Mass Spectrometer. Analytical Chemistry, 2014. 86(4): p. 2107-2116.

31. Paglia, G., et al., Ion Mobility Derived Collision Cross Sections to Support Metabolomics Applications. Analytical Chemistry, 2014. 86(8): p. 3985-3993.

32. Zhang, X., et al., Metabolic Analysis of Striatal Tissues from Parkinson's Disease-like Rats by Electrospray Ionization Ion Mobility Mass Spectrometry. Analytical Chemistry, 2014. 
33. Yassin, G.H., et al., Investigation of isomeric flavanol structures in black tea thearubigins using ultraperformance liquid chromatography coupled to hybrid quadrupole/ion mobility/time of flight mass spectrometry. Journal of Mass Spectrometry, 2014. 49(11): p. 1086-1095.

34. Malkar, A., et al., Metabolic profiling of human saliva before and after induced physiological stress by ultra-high performance liquid chromatography-ion mobility-mass spectrometry. Metabolomics, 2013. 9(6): p. 1192-1201.

35. Patti, G.J., O. Yanes, and G. Siuzdak, Metabolomics: the apogee of the omics trilogy. Nature Reviews Molecular Cell Biology, 2012. 13(4): p. 263-269.

36. Rappaport, S.M., Implications of the exposome for exposure science. Journal of Exposure Science and Environmental Epidemiology, 2011. 21(1): p. 5-9.

37. Weckwerth, W., Metabolomics in systems biology. Annual Review of Plant Biology, 2003. 54: p. 669-689.

38. Lawton, K.A., et al., Analysis of the adult human plasma metabolome. Pharmacogenomics, 2008. 9(4): p. 383-97.

39. Czene, K., P. Lichtenstein, and K. Hemminki, Environmental and heritable causes of cancer among 9.6 million individuals in the Swedish family-cancer database. International Journal of Cancer, 2002. 99(2): p. 260-266.

40. Menni, C., et al., Metabolomic markers reveal novel pathways of ageing and early development in human populations. International Journal of Epidemiology, 2013. 42(4): p. 1111-1119.

41. Rhee, E.P. and R.E. Gerszten, Metabolomics and Cardiovascular Biomarker Discovery. Clinical Chemistry, 2012. 58(1): p. 139-147.

42. Spratlin, J.L., N.J. Serkova, and S.G. Eckhardt, Clinical Applications of Metabolomics in Oncology: A Review. Clinical Cancer Research, 2009. 15(2): p. 431-440.

43. Underwood, B.R., et al., Huntington disease patients and transgenic mice have similar pro-catabolic serum metabolite profiles. Brain, 2006. 129: p. 877-886.

44. Bogdanov, M., et al., Metabolomic profiling to develop blood biomarkers for Parkinson's disease. Brain, 2008. 131: p. 389-396.

45. Li, F., W. Xu, and S.M. Zhao, Regulatory Roles of Metabolites in Cell Signaling Networks. Journal of Genetics and Genomics, 2013. 40(7): p. 367-374.

46. Cheng, S.S., et al., Metabolite Profiling Identifies Pathways Associated With Metabolic Risk in Humans. Circulation, 2012. 125(18): p. 2222-U132.

47. Dwivedi, P., A.J. Schultz, and H.H. Hill, Metabolic profiling of human blood by high-resolution ion mobility mass spectrometry (IM-MS). International Journal of Mass Spectrometry, 2010. 298(1-3): p. 78-90.

48. Wishart, D.S., et al., HMDB: the Human Metabolome Database. Nucleic Acids Res, 2007. 35(Database issue): p. D521-6.

49. Patti, G.J., R. Tautenhahn, and G. Siuzdak, Meta-analysis of untargeted metabolomic data from multiple profiling experiments. Nature Protocols, 2012. 7(3): p. 508-516.

50. Bocker, S. and F. Rasche, Towards de novo identification of metabolites by analyzing tandem mass spectra. Bioinformatics, 2008. 24(16): p. 149-155.

51. Scheubert, K., et al., Computing fragmentation trees from metabolite multiple mass spectrometry data. J Comput Biol, 2011. 18(11): p. 1383-97. 
52. Cao, M., K. Fraser, and S. Rasmussen, Computational Analyses of Spectral Trees from Electrospray Multi-Stage Mass Spectrometry to Aid Metabolite Identification. Metabolites, 2013. 3(4): p. 1036-1050.

53. Peterson, A.C., et al., Parallel reaction monitoring for high resolution and high mass accuracy quantitative, targeted proteomics. Mol Cell Proteomics, 2012. 11(11): p. 1475-88.

54. Gillet, L.C., et al., Targeted data extraction of the MS/MS spectra generated by data-independent acquisition: a new concept for consistent and accurate proteome analysis. Mol Cell Proteomics, 2012. 11(6): p. O111.016717.

55. Hoadlund-Hyzer, C.S., J.W. Li, and D.E. Clemmer, Mobility labeling for parallel CID of ion mixtures. Analytical Chemistry, 2000. 72(13): p. 2737-2740.

56. Koeniger, S.L., et al., An IMS-IMS analogue of MS-MS. Analytical Chemistry, 2006. 78(12): p. 4161-4174.

57. Lee, S., et al., Extracted Fragment Ion Mobility Distributions: A New Method for Complex Mixture Analysis. Int J Mass Spectrom, 2012. 309: p. 154-160.

58. Zubarev, R.A., N.L. Kelleher, and F.W. McLafferty, Electron Capture Dissociation of Multiply Charged Protein Cations. A Nonergodic Process. Journal of the American Chemical Society, 1998. 120(13): p. 3265-3266.

59. Zhurov, K.O., et al., Principles of electron capture and transfer dissociation mass spectrometry applied to peptide and protein structure analysis. Chemical Society Reviews, 2013. 42(12): p. 5014-5030.

60. Massonnet, P., et al., Combined use of ion mobility and collision-induced dissociation to investigate the opening of disulfide bridges by electron-transfer dissociation in peptides bearing two disulfide bonds. Anal Chem, 2015. 87(10): p. 5240-6.

61. Williams, J.P., et al., Identifying drug metallation sites on peptides using electron transfer dissociation (ETD), collision induced dissociation (CID) and ion mobilitymass spectrometry (IM-MS). Chemical Communications, 2010. 46(30): p. 54585460.

62. Rand, K.D., et al., Site-Specific Analysis of Gas-Phase Hydrogen/Deuterium Exchange of Peptides and Proteins by Electron Transfer Dissociation. Analytical Chemistry, 2012. 84(4): p. 1931-1940.

63. Rand, K.D., et al., Gas-phase hydrogen/deuterium exchange in a traveling wave ion guide for the examination of protein conformations. Anal Chem, 2009. 81(24): p. 10019-28.

64. Waters. SYNAPT G2-Si High Definition Mass Spectrometry. 2014 [cited 2014 7/1]; Available from: http://www.waters.com/waters/en US/SYNAPT-G2-Si-HighDefinition-Mass-Spectrometry/nav.htm?cid=134740622\&locale=en US.

65. May, J.C. and J.A. McLean, Ion Mobility-Mass Spectrometry: Time-Dispersive Instrumentation. Analytical Chemistry, 2014.

66. Xuan, Y., et al., High-field asymmetric waveform ion mobility spectrometry (FAIMS) coupled with high-resolution electron transfer dissociation mass spectrometry for the analysis of isobaric phosphopeptides. Rapid Commun Mass Spectrom, 2009. 23(13): p. 1963-9.

67. Cohen, P., The regulation of protein function by multisite phosphorylation--a 25 year update. Trends Biochem Sci, 2000. 25(12): p. 596-601. 
68. Johnson, L.N., The regulation of protein phosphorylation. Biochem Soc Trans, 2009. 37(Pt 4): p. 627-41.

69. Thorner, J., et al., Signal transduction: From the atomic age to the post-genomic era. Cold Spring Harb Perspect Biol, 2014. 6(12): p. a022913.

70. Riley, N.M. and J.J. Coon, Phosphoproteomics in the Age of Rapid and Deep Proteome Profiling. Analytical Chemistry, 2016. 88(1): p. 74-94.

71. Palumbo, A.M., et al., Tandem mass spectrometry strategies for phosphoproteome analysis. Mass Spectrometry Reviews, 2011. 30(4): p. 600625.

72. Sharma, K., et al., Ultradeep Human Phosphoproteome Reveals a Distinct Regulatory Nature of Tyr and Ser/Thr-Based Signaling. Cell Reports, 2014. 8(5): p. 1583-1594.

73. Huttlin, E.L., et al., A tissue-specific atlas of mouse protein phosphorylation and expression. Cell, 2010. 143(7): p. 1174-89.

74. Mertins, P., et al., iTRAQ labeling is superior to $m T R A Q$ for quantitative global proteomics and phosphoproteomics. Mol Cell Proteomics, 2012. 11(6): $\mathrm{p}$. M111.014423.

75. Jedrychowski, M.P., et al., Evaluation of HCD-and CID-type fragmentation within their respective detection platforms for murine phosphoproteomics. Mol Cell Proteomics, 2011. 10(12): p. M111.009910.

76. Collins, M.O., et al., Confident and sensitive phosphoproteomics using combinations of collision induced dissociation and electron transfer dissociation. J Proteomics, 2014. 103: p. 1-14.

77. Wiese, $\mathrm{H}$., et al., Comparison of Alternative MS/MS and Bioinformatics Approaches for Confident Phosphorylation Site Localization. Journal of Proteome Research, 2014. 13(2): p. 1128-1137.

78. Good, D.M., et al., Performance characteristics of electron transfer dissociation mass spectrometry. Mol Cell Proteomics, 2007. 6(11): p. 1942-51.

79. Hunt, D.F., J. Shabanowitz, and D.L. Bai, Peptide Sequence Analysis by Electron Transfer Dissociation Mass Spectrometry: A Web-Based Tutorial. J Am Soc Mass Spectrom, 2015. 26(7): p. 1256-8.

80. Konermann, L., J. Pan, and Y.-H. Liu, Hydrogen exchange mass spectrometry for studying protein structure and dynamics. Chemical Society Reviews, 2011. 40(3): p. 1224-1234.

81. Wales, T.E. and J.R. Engen, Hydrogen exchange mass spectrometry for the analysis of protein dynamics. Mass Spectrometry Reviews, 2006. 25(1): p. 158170.

82. Englander, S.W., Hydrogen Exchange and Mass Spectrometry: A Historical Perspective. Journal of the American Society for Mass Spectrometry, 2006. 17(11): p. 1481-1489.

83. Zhang, Z.Q. and D.L. Smith, DETERMINATION OF AMIDE HYDROGENEXCHANGE BY MASS-SPECTROMETRY - A NEW TOOL FOR PROTEINSTRUCTURE ELUCIDATION. Protein Science, 1993. 2(4): p. 522-531.

84. Hamuro, Y., et al., Rapid analysis of protein structure and dynamics by hydrogen/deuterium exchange mass spectrometry. Journal of biomolecular techniques : JBT, 2003. 14(3): p. 171-82. 
85. Englander, S.W., et al., Mechanisms and uses of hydrogen exchange. Current Opinion in Structural Biology, 1996. 6(1): p. 18-23.

86. Engen, J.R., Analysis of Protein Conformation and Dynamics by Hydrogen/Deuterium Exchange MS. Analytical Chemistry, 2009. 81(19): p. 78707875.

87. Kaltashov, I.A., C.E. Bobst, and R.R. Abzalimov, H/D Exchange and Mass Spectrometry in the Studies of Protein Conformation and Dynamics: Is There a Need for a Top-Down Approach? Analytical Chemistry, 2009. 81(19): p. 78927899.

88. Kaltashov, I.A., C.E. Bobst, and R.R. Abzalimov, Mass spectrometry-based methods to study protein architecture and dynamics. Protein Science, 2013. 22(5): p. 530-544.

89. Keppel, T.R. and D.D. Weis, Analysis of Disordered Proteins Using a Simple Apparatus for Millisecond Quench-Flow H/D Exchange. Analytical Chemistry, 2013. 85(10): p. 5161-5168.

90. Skinner, J.J., et al., Protein dynamics viewed by hydrogen exchange. Protein Sci, 2012. 21(7): p. 996-1005.

91. Katta, V. and B.T. Chait, CONFORMATIONAL-CHANGES IN PROTEINS PROBED BY HYDROGEN-EXCHANGE ELECTROSPRAY-IONIZATION MASSSPECTROMETRY. Rapid Communications in Mass Spectrometry, 1991. 5(4): p. 214-217.

92. Lee, T., et al., Docking Motif Interactions in MAP Kinases Revealed by Hydrogen Exchange Mass Spectrometry. Molecular Cell, 2004. 14(1): p. 43-55.

93. Ehring, H., Hydrogen Exchange/Electrospray lonization Mass Spectrometry Studies of Structural Features of Proteins and Protein/Protein Interactions. Analytical Biochemistry, 1999. 267(2): p. 252-259.

94. Sowole, M.A., et al., Noncovalent binding of a cyclic peptide inhibitor to the peptidyl-prolyl isomerase Pin1, explored by hydrogen exchange mass spectrometry. Canadian Journal of Chemistry, 2014. 93(1): p. 44-50.

95. Sowole, M.A. and L. Konermann, Effects of Protein-Ligand Interactions on Hydrogen/Deuterium Exchange Kinetics: Canonical and Noncanonical Scenarios. Analytical Chemistry, 2014. 86(13): p. 6715-6722.

96. Arndt, J.R., et al., Lysine residues in the $N$-terminal huntingtin amphipathic $\alpha$ helix play a key role in peptide aggregation. Journal of Mass Spectrometry, 2015. 50(1): p. 117-126.

97. Weis, D.D., et al., Identification and Characterization of EX1 Kinetics in $H / D$ Exchange Mass Spectrometry by Peak Width Analysis. Journal of the American Society for Mass Spectrometry, 2006. 17(11): p. 1498-1509.

98. Sivaraman, T. and A. Robertson, Kinetics of Conformational Fluctuations by EX1 Hydrogen Exchange in Native Proteins, in Protein Structure, Stability, and Folding, K. Murphy, Editor. 2001, Humana Press. p. 193-214.

99. Krishna, M.M., et al., Hydrogen exchange methods to study protein folding. Methods, 2004. 34(1): p. 51-64.

100. Mayne, L., et al., Many Overlapping Peptides for Protein Hydrogen Exchange Experiments by the Fragment Separation-Mass Spectrometry Method. Journal of The American Society for Mass Spectrometry, 2011. 22(11): p. 1898-1905. 
101. Zhang, H.M., et al., Fast reversed-phase liquid chromatography to reduce back exchange and increase throughput in $H / D$ exchange monitored by FT-ICR mass spectrometry. J Am Soc Mass Spectrom, 2009. 20(3): p. 520-4.

102. Jones, L.M., et al., Online, High-Pressure Digestion System for Protein Characterization by Hydrogen/Deuterium Exchange and Mass Spectrometry. Analytical Chemistry, 2010. 82(4): p. 1171-1174.

103. Engen, J.R. and D.L. Smith, Investigating protein structure and dynamics by hydrogen exchange MS. Anal Chem, 2001. 73(9): p. 256a-265a.

104. Ahn, J., et al., Accessing the reproducibility and specificity of pepsin and other aspartic proteases. Biochim Biophys Acta, 2013. 1834(6): p. 1222-9.

105. Ahn, J., et al., Pepsin immobilized on high-strength hybrid particles for continuous flow online digestion at 10,000 psi. Anal Chem, 2012. 84(16): p. 7256-62.

106. Pan, J., et al., Electron Capture Dissociation of Electrosprayed Protein lons for Spatially Resolved Hydrogen Exchange Measurements. Journal of the American Chemical Society, 2008. 130(35): p. 11574-11575.

107. Huang, R.Y.C., et al., Hydrogen/Deuterium Exchange and Electron-Transfer Dissociation Mass Spectrometry Determine the Interface and Dynamics of Apolipoprotein E Oligomerization. Biochemistry, 2011. 50(43): p. 9273-9282.

108. Zehl, M., et al., Electron Transfer Dissociation Facilitates the Measurement of Deuterium Incorporation into Selectively Labeled Peptides with Single Residue Resolution. Journal of the American Chemical Society, 2008. 130(51): p. 1745317459.

109. Pan, J. and C.H. Borchers, Top-down structural analysis of posttranslationally modified proteins by Fourier transform ion cyclotron resonance-MS with hydrogen/deuterium exchange and electron capture dissociation. Proteomics, 2013. 13(6): p. 974-81.

110. Abzalimov, R.R., C.E. Bobst, and I.A. Kaltashov, A New Approach to Measuring Protein Backbone Protection with High Spatial Resolution Using H/D Exchange and Electron Capture Dissociation. Analytical Chemistry, 2013. 85(19): p. 91739180.

111. Rand, K.D., et al., Protein hydrogen exchange measured at single-residue resolution by electron transfer dissociation mass spectrometry. Anal Chem, 2009. 81(14): p. 5577-84.

112. Landgraf, R., M. Chalmers, and P. Griffin, Automated Hydrogen/Deuterium Exchange Electron Transfer Dissociation High Resolution Mass Spectrometry Measured at Single-Amide Resolution. Journal of The American Society for Mass Spectrometry, 2012. 23(2): p. 301-309.

113. Rand, K.D., et al., Protein Hydrogen Exchange Measured at Single-Residue Resolution by Electron Transfer Dissociation Mass Spectrometry. Analytical Chemistry, 2009. 81(14): p. 5577-5584.

114. Deng, Y., H. Pan, and D.L. Smith, Selective isotope labeling demonstrates that hydrogen exchange at individual peptide amide linkages can be determined by collision-induced dissociation mass spectrometry. Journal of the American Chemical Society, 1999. 121(9): p. 1966-1967. 
115. Abzalimov, R.R. and I.A. Kaltashov, Controlling Hydrogen Scrambling in Multiply Charged Protein lons during Collisional Activation: Implications for Top-Down Hydrogen/Deuterium Exchange MS Utilizing Collisional Activation in the Gas Phase. Analytical Chemistry, 2010. 82(3): p. 942-950.

116. Hoerner, J.K., H. Xiao, and I.A. Kaltashov, Structural and Dynamic Characteristics of a Partially Folded State of Ubiquitin Revealed by Hydrogen Exchange Mass Spectrometryt. Biochemistry, 2005. 44(33): p. 11286-11294.

117. Abzalimov, R.R., et al., Protein conformations can be probed in top-down HDX MS experiments utilizing electron transfer dissociation of protein ions without hydrogen scrambling. Journal of the American Society for Mass Spectrometry, 2009. 20(8): p. 1514-1517.

118. Rand, K.D., et al., Electron capture dissociation proceeds with a low degree of intramolecular migration of peptide amide hydrogens. J Am Chem Soc, 2008. 130(4): p. 1341-9.

119. Rand, K.D., M. Zehl, and T.J. Jorgensen, Measuring the hydrogen/deuterium exchange of proteins at high spatial resolution by mass spectrometry: overcoming gas-phase hydrogen/deuterium scrambling. Acc Chem Res, 2014. 47(10): p. 3018-27.

120. Syka, J.E., et al., Peptide and protein sequence analysis by electron transfer dissociation mass spectrometry. Proceedings of the National Academy of Sciences of the United States of America, 2004. 101(26): p. 9528-9533.

121. Baker, E.S., et al., Ion mobility spectrometry-mass spectrometry performance using electrodynamic ion funnels and elevated drift gas pressures. J Am Soc Mass Spectrom, 2007. 18(7): p. 1176-87.

122. Becker, C., F.A. Fernandez-Lima, and D.H. Russell, Ion Mobility-Mass Spectrometry: A Tool for Characterizing the Petroleome. Spectroscopy, 2009. 24(4): p. 38-42.

123. Clowers, B.H., et al., Separation of sodiated isobaric disaccharides and trisaccharides using electrospray ionization-atmospheric pressure ion mobilitytime of flight mass spectrometry. Journal of the American Society for Mass Spectrometry, 2005. 16(5): p. 660-669.

124. Ruotolo, B.T., et al., Peak capacity of ion mobility mass spectrometry: Separation of peptides in helium buffer gas. Journal of Chromatography B-Analytical Technologies in the Biomedical and Life Sciences, 2002. 782(1-2): p. 385-392.

125. Valentine, S.J., et al., Toward Plasma Proteome Profiling with lon Mobility-Mass Spectrometry. Journal of Proteome Research, 2006. 5(11): p. 2977-2984.

126. Wu, C., et al., Separation of isomeric peptides using electrospray ionization/highresolution ion mobility spectrometry. Anal Chem, 2000. 72(2): p. 391-5.

127. Bohrer, B.C., et al., Biomolecule Analysis by Ion Mobility Spectrometry, in Annual Review of Analytical Chemistry. 2008. p. 293-327.

128. Clemmer, D.E. and M.F. Jarrold, Ion mobility measurements and their applications to clusters and biomolecules. Journal of Mass Spectrometry, 1997. 32(6): p. 577-592.

129. Counterman, A.E., et al., Formation of peptide aggregates during ESI: Size, charge, composition, and contributions to noise. Journal of the American Society for Mass Spectrometry, 2001. 12(9): p. 1020-1035. 
130. Zinnel, N.F., P.J. Pai, and D.H. Russell, Ion Mobility-Mass Spectrometry (IM-MS) for Top-Down Proteomics: Increased Dynamic Range Affords Increased Sequence Coverage. Analytical Chemistry, 2012. 84(7): p. 3390-3397.

131. Rand, K., et al., ETD in a Traveling Wave Ion Guide at Tuned Z-Spray Ion Source Conditions Allows for Site-Specific Hydrogen/Deuterium Exchange Measurements. Journal of The American Society for Mass Spectrometry, 2011. 22(10): p. 1784-1793.

132. lacob, R.E., J.P. Murphy, and J.R. Engen, Ion mobility adds an additional dimension to mass spectrometric analysis of solution-phase hydrogen/deuterium exchange. Rapid Communications in Mass Spectrometry, 2008. 22(18): p. 28982904.

133. Mistarz, U.H., et al., Simple Setup for Gas-Phase H/D Exchange Mass Spectrometry Coupled to Electron Transfer Dissociation and lon Mobility for Analysis of Polypeptide Structure on a Liquid Chromatographic Time Scale. Analytical Chemistry, 2014. 86(23): p. 11868-11876.

134. Beeston, H.S., et al., Changes in protein structure monitored by use of gasphase hydrogen/deuterium exchange. Proteomics, 2015.

135. Khakinejad, M., et al., Combining ion mobility spectrometry with hydrogendeuterium exchange and top-down MS for peptide ion structure analysis. J Am Soc Mass Spectrom, 2014. 25(12): p. 2103-15.

136. Khakinejad, M., et al., Gas-Phase Hydrogen-Deuterium Exchange Labeling of Select Peptide Ion Conformer Types: a Per-Residue Kinetics Analysis. J Am Soc Mass Spectrom, 2015.

137. Campbell, S., et al., Deuterium exchange reactions as a probe of biomolecule structure. Fundamental studies of cas phase $H / D$ exchange reactions of protonated glycine oligomers with $D 20, C D 3 O D, C D 3 C O 2 D$, and ND3. Journal of the American Chemical Society, 1995. 117(51): p. 12840-12854.

138. Gard, E., et al., Gas-phase hydrogen/deuterium exchange as a molecular probe for the interaction of methanol and protonated peptides. J Am Soc Mass Spectrom, 1994. 5(7): p. 623-31.

139. Campbell, S., et al., Deuterium Exchange Reactions as a Probe of Biomolecule Structure. Fundamental Studies of Gas Phase H/D Exchange Reactions of Protonated Glycine Oligomers with D2O, CD3OD, CD3CO2D, and ND3. Journal of the American Chemical Society, 1995. 117(51): p. 12840-12854.

140. Suckau, D., et al., Coexisting stable conformations of gaseous protein ions. Proceedings of the National Academy of Sciences of the United States of America, 1993. 90(3): p. 790-793.

141. Chan, S. and C.G. Enke, Mechanistic study of hydrogen/deuterium exchange between [M-1]- ions of chlorinated benzenes and D2O or ND3. Journal of the American Society for Mass Spectrometry, 1994. 5(4): p. 282-291.

142. Robinson, J.M., et al., Hydrogen/deuterium exchange of nucleotides in the gas phase. Analytical Chemistry, 1998. 70(17): p. 3566-3571.

143. Freitas, M.A., et al., Gas-phase RNA and DNA ions. 1. H/D exchange of the $M-H$ (-) anions of nucleoside 5 '-monophosphates (GMP, dGMP, AMP, dAMP, CMP, dCMP, UMP, dTMP), ribose 5-monophosphate, and 2-deoxyribose 5- 
monophosphate with D2O and D2S. Journal of the American Chemical Society, 1998. 120(39): p. 10187-10193.

144. Chipuk, J.E. and J.S. Brodbelt, Gas-phase hydrogen/deuterium exchange of 5 'and 3 '-mononucleotides in a quadrupole ion trap: Exploring the role of conformation and system energy. Journal of the American Society for Mass Spectrometry, 2007. 18(4): p. 724-736.

145. Freitas, M.A. and A.G. Marshall, Gas phase RNA and DNA ions 2. Conformational dependence of the gas-phase H/D exchange of nucleotide-5 'monophosphates. Journal of the American Society for Mass Spectrometry, 2001. 12(7): p. 780-785.

146. Crestoni, M.E. and S. Fornarini, Gas-phase hydrogen/deuterium exchange of adenine nucleotides. Journal of Mass Spectrometry, 2003. 38(8): p. 854-861.

147. Tian, Z., L. Lis, and S.R. Kass, Hydrogen-deuterium exchange and selective labeling of deprotonated amino acids and peptides in the gas phase. J Am Chem Soc, 2008. 130(1): p. 8-9.

148. Bache, N., et al., Hydrogen atom scrambling in selectively labeled anionic peptides upon collisional activation by MALDI tandem time-of-flight mass spectrometry. J Am Soc Mass Spectrom, 2008. 19(12): p. 1719-25.

149. Yoo, H.J., et al., Negative-ion electron capture dissociation: radical-driven fragmentation of charge-increased gaseous peptide anions. J Am Chem Soc, 2011. 133(42): p. 16790-3.

150. Hersberger, K.E. and K. Hakansson, Characterization of O-Sulfopeptides by Negative Ion Mode Tandem Mass Spectrometry: Superior Performance of Negative Ion Electron Capture Dissociation. Analytical Chemistry, 2012. 84(15): p. 6370-6377.

151. Cook, S.L., et al., Comparison of CID, ETD and metastable atom-activated dissociation (MAD) of doubly and triply charged phosphorylated tau peptides. Journal of Mass Spectrometry, 2012. 47(6): p. 786-794.

152. Cook, S.L., O.L. Collin, and G.P. Jackson, Metastable atom-activated dissociation mass spectrometry: leucine/isoleucine differentiation and ring cleavage of proline residues. Journal of Mass Spectrometry, 2009. 44(8): $p$. 1211-1223.

153. Zhou, M.W. and V.H. Wysocki, Surface Induced Dissociation: Dissecting Noncovalent Protein Complexes in the Gas phase. Accounts of Chemical Research, 2014. 47(4): p. 1010-1018.

154. Zhou, M.W., C.S. Huang, and V.H. Wysocki, Surface-Induced Dissociation of Ion Mobility-Separated Noncovalent Complexes in a Quadrupole/Time-of-Flight Mass Spectrometer. Analytical Chemistry, 2012. 84(14): p. 6016-6023.

155. Hoffmann, W. and G. Jackson, Charge Transfer Dissociation (CTD) Mass Spectrometry of Peptide Cations Using Kiloelectronvolt Helium Cations. Journal of The American Society for Mass Spectrometry, 2014. 25(11): p. 1939-1943.

156. Chingin, K., et al., Fragmentation of Positively-Charged Biological lons Activated with a Beam of High-Energy Cations. Analytical Chemistry, 2014. 86(1): p. 372379 . 


\section{A New Ion Mobility - Linear Ion Trap Instrument for Complex Mixture Analysis ${ }^{1}$}

${ }^{1}$ Reprinted in part with permission from Analytical Chemistry: A New lon Mobility - Linear lon Trap for Complex Mixture Analysis. Gregory C. Donohoe, Hossein Maleki, James R. Arndt, Mahdiar Khakinejad, Jinghai Yi, Carroll McBride, Timothy Nurkiewicz, and Stephen J. Valentine. Anal. Chem., 2014, 86 (16), pp 8121-8128.

\subsection{Introduction: Complex Mixture Separations Using IMS-MS}

Over the last two decades, the use of ion mobility spectrometry (IMS) coupled with mass spectrometry (MS) as a means for biomolecular ion separation has grown dramatically.[1-13] The added peak capacity afforded by the gas-phase separation (IMS) enhances the detection of lower-signal species by removing them from spectral regions containing interfering features from higher-signal ions.[7, 14-16] This advantage has driven technological development in the private sector as an increasing number of MS-based instrument platforms are adopting/developing IMS separation steps.[17-19]

Much of the IMS-MS instrumentation demonstrated for complex mixture analysis has employed the use of time-of-flight (TOF) mass analyzers.[1, 2, 20] The advantage of coupling IMS with TOFMS is that individual TOF spectra can be "nested" within the drift time $\left(t_{D}\right)$ measurement.[21] Although this instrument configuration allows the mass determination of all mobility-dispersed ions, the inability to trap ions precludes the use of multi-stage tandem mass spectrometry $\left(\mathrm{MS}^{\mathrm{n}}\right)$ as a means for identifying complex mixture components. This is problematic for identifying intractable molecules for which MS/MS analysis is insufficient.[22-25]

IMS-MS instrumentation development has also yielded instruments combining drift tubes with mass spectrometers that employ ion trapping.[26-28][29, 30] Featured designs include those utilizing high-resolution drift tubes operated at atmospheric 
pressure and elevated drift voltages.[27, 28] Two challenges became evident with this type of instrument design that relate to the overall measurement sensitivity. The first challenge was associated with the low duty cycle of the measurement. The second was that the addition of the required mobility-selection ion gate essentially resulted in a scanning/filtering operational mode. That is, only ions of select mobilities were transferred into the mass spectrometer. These problems may be addressed in the future using ideas presented in seminal studies demonstrating the use of Fourier Transform and Hadamard Transform IMS.[31-33]

One problem that persists with instruments coupling high-pressure IMS and ion trap mass spectrometers is the decrease in ion transmission across the drift tube-mass spectrometer interface region. That is, ions can be lost in conductance-limiting regions as there are no means currently available to focus diffuse ion clouds at elevated pressures. The ability to compress ion packets in low-pressure drift tubes[34, 35] was one of the motivating factors for pursuing the instrumentation design described here. Additionally, the low-pressure drift tube can not only serve as the device to achieve gasphase separation but also as an ion fragmentation cell employing collision-induced dissociation (CID).[36] Indeed, the higher-pressure fragmentation process (hereafter referred to as IMS-CID) has been demonstrated to be efficient and highly tunable.[7, 3638] The combination of the IMS-CID capabilities of the drift tube with the MS ${ }^{n}$ capabilities of the linear ion trap further distinguish the instrument described here.

The new analytical capabilities of the instrument are here demonstrated with the analysis of two model mixture samples. The first sample analysis represents the capability to rapidly identify phosphorylated peptides in tryptic digests such as would be 
encountered in post-translational modification (PTM) experiments. For these studies, IMS-CID methods show that targets for PTM analysis can rapidly be ascertained and subsequently subjected to electron transfer dissociation (ETD)[39] to identify phosphorylation sites. To some degree, the approach is similar to proteomics techniques that scan for neutral loss of $\mathrm{H}_{3} \mathrm{PO}_{4}$ and subject target ions to further tandem MS experiments.[40, 41] A difference is that extracted ion drift time distributions (XIDTD)[42] help to confirm and isolate ions for subsequent tandem MS experiments. Although several instruments demonstrating fragmentation of mobility-selected ions have been described,[7, 36, 37, 43, 44] this work presents the first combination of IMSCID[36, 38] and ETD-MS. The second experimental example using the IM-MS to profile the plasma metabolome of rats exposed to engineered nanomaterials (ENM) via inhalation. For these studies organisms are exposed to $\mathrm{TiO}_{2}$ nanoparticles (NP). Here NP can be described as species exhibiting less than $100 \mathrm{~nm}$ in length in any dimension. Two-dimensional (2D) IMS-MS datasets are generated for samples from organisms for different post exposure times (directly after and 24 hours post exposure). Comparisons of the 2D datasets show the enhancement provided by the IMS separation for distinguishing samples. Instrumental aspects facilitating ion identification and relative quantitation including IMS coupled with $\mathrm{MS}^{\mathrm{n}}$ and IM-collision-induced dissociation (CID)-MS[36, 43] are discussed This study is unique in that it presents the first demonstration of IMS-MS ${ }^{n}$ to putatively identify plasma metabolites in comparative analyses. Both 'omic analyses demonstrate the ability of the IM-MS separation compare all spectra without dataset alignment. 
The described instrument is similar to that coupling IMS-LIT analysis with photodissocation capabilities;[29, 42] however, this work describes unique ion fragmentation techniques such as the combination of two different methods (IMS-CID and ETD-MS) as well as $\mathrm{MS}^{4}$ of mobility-selected ions. The direction of future instrumentation development is discussed while considering improvements for complex mixture analysis.

\subsection{Materials and Methods}

Cytochrome $c$ (Equine, 95\%) was purchased from Sigma Aldrich (St. Louis, MO) and used without further purification. Phosphorylated peptide standards (95\% purity) with sequences of KRPsQRHGSKY-NH2 and SFVLNPTNIGMsKSSQGHVTK were purchased from AnaSpec (Fenton CA) and used without further purification. The peptide, KKDDDDDIIKIIK ( 90\%)was purchased from Genscript. Ultra-pure (chromatography grade) deionized water, methanol, and formic acid (Fisher Scientific, Fair Lawn, NJ, USA) were used to generate stock and ESI solutions of the peptide and proteins.

\subsubsection{Phosphoproteomic Samples}

Cytochrome $c(1.0 \mathrm{mg})$ was diluted in $1.0 \mathrm{~mL}$ of deionized water. Urea was added to a final concentration of $1.5 \mathrm{M}$. TPCK-treated trypsin was added at a ratio of 1:20 (trypsin:cytochrome $c$ ) and the sample was incubated overnight. The tryptic digest $(100 \mu \mathrm{L})$ was diluted 10 fold in ESI solution (1:1 water:methanol and 1\% formic acid)

and $10 \mu \mathrm{g}\left(0.01 \mu \mathrm{g} \cdot \mathrm{LL}^{-1}\right)$ of the phosphorylated peptide standard (KRPsQRHGSKY$\mathrm{NH} 2$ ) was added to the solution.

\subsubsection{Rat Exposure to $\mathrm{TiO}_{2}$ Nanoparticles}


Rat plasma samples were obtained from the West Virginia University Center for Cardiovascular and Respiratory Sciences, School of Medicine, Morgantown, WV. The ENM exposure apparatus and organism handling were described in detail previously.[45, 46] Briefly, Male Sprague Dawley rats (175 - $250 \mathrm{~g})$ were purchased from Hilltop Laboratories (Scottsdale, PA), and housed in laminar flow cages under controlled temperature and humidity conditions and a $12 \mathrm{hr} \mathrm{light} / 12 \mathrm{hr}$ dark cycle at the West Virginia University Health Sciences Center vivarium. Food and water were provided ad libitum. All animals were acclimated for 72 -hours before nanomaterial inhalation exposure. To ensure that all methods were performed humanely and with regard to alleviation of suffering, all procedures were approved by the Institutional Animal Care and Use Committee of West Virginia University.

To profile the metabolomes at different post-exposure times, blood was collected directly after exposure to $\mathrm{TiO}^{2}$ nanoparticles and 24 hours post exposure for control (filtered air) and exposed animals. During exposure, rats were placed into a home-built chamber specifically designed to deliver the nanoparticles. An aerosolized stream of $\mathrm{TiO}_{2}$ was introduced into the chamber for three hours to deliver an estimated pulmonary load of $30 \mu \mathrm{g}$ per animal. Blood samples were immediately centrifuged for 10 minutes at $2500 \mathrm{~g}$ to produce plasma. The plasma was stored at $-80^{\circ} \mathrm{C}$ until preparation. Hereafter, plasma samples generated directly after exposure and 24 hours post exposure is referred to as EXP0 and EXP24, respectively. For control animals, the same time point samples are referred to as CNTRL0 and CNTRL24.

\subsubsection{Metabolite Extraction}


Pooled plasma samples were prepared from the 40 individual samples by combining $25-\mu \mathrm{L}$ aliquots. Using a temperature-controlled micro-centrifuge digital dry bath (Lanbnet International, Inc, Edison, NJ, USA), $400 \mu \mathrm{L}$ of $80^{\circ} \mathrm{C}$ methanol is added to $100 \mu \mathrm{L}$ of pooled sample. The samples were vortexed and incubated first at $80^{\circ} \mathrm{C}(5$ mintues) and immediately transferred to ice (5 minutes). Proteins were pelleted using centrifugation (ThermoScientific, San Jose, CA, USA) at 13,000 $\mathrm{g}$ for 20 minutes at 4 ${ }^{\circ} \mathrm{C}$. The supernantuant $(\sim 375 \mu \mathrm{L})$ was combined with $300 \mu \mathrm{L} 18 \mathrm{M} \Omega \mathrm{H}_{2} \mathrm{O}: 5 \%$ formic acid. Samples were delivered by a syringe pump (KD scientific Holliston, MA, USA) using a $500-\mu \mathrm{L}$ syringe (Hamilton, Reno, NV, USA) to a pulled-tip nano-electrospray emitter $(350 \mu \mathrm{m}$ o.d. $\times 75 \mu \mathrm{m}$ i.d. $)$. The sample flow was maintained at $300 \mathrm{~nL} \cdot \mathrm{min}^{-1}$ and a bias of $2.2 \mathrm{kV}$ was used to perform electrospray ionization (ESI)[47] of the metabolite mixture.

\subsubsection{IMS-MS: Instrumental Operation}

IMS theory,[48-51] instrumentation[32, 52-58] and techniques[43, 59-62] are described elsewhere in great detail. For these experiments, the IMS-MS instrument consists of a dual ion funnel/ ion gate design coupled to a LTQ Velos (Thermo Electron, San Jose, CA, USA) mass spectrometer similar to that reported previously.[29, 42] Figure 1.1 shows the instrument schematic designating the locations of the ion funnels, ion gates and ion activation regions within the drift tube. The mass spectrometer has been modified by replacing the ion source and s-lens assembly with a stacked-ring ion drift region (Figure 1.1). Briefly, electrosprayed ions enter into a desolvation region and are focused through an hour-glass ion funnel (F1 in Figure 1.1).[13, 63] lons are trapped in F1 and periodically pulsed into the drift tube. The drift tube is filled with He buffer gas 
( 2.5 Torr at $300 \mathrm{~K})$. lons traverse the drift tube under the influence of a uniform electric field $\left(\sim 11 \mathrm{~V} \cdot \mathrm{cm}^{-1}\right)$ and separate based on differences in their mobilities. Upon reaching a selection gate (G2 in Figure 1.1) ions pass into a second ion funnel (F2) used to radially focus the ion cloud.[34, 35] The ions subsequently exit the drift tube through a conductance-limiting aperture and are focused by quadrupole and octopole rf guides before entering into the higher pressure linear ion trap followed by subsequent mass analysis in the lower pressure trap.

\subsubsection{Recording Two-Dimensional (2D) $t_{\underline{D}}(\mathrm{~m} / \mathrm{z})$ Distributions.}

lon gates (Figure 1.1) are constructed using Ni gridded lens (90\% transmittance mesh; Precision Eforming, Cortland, NY, USA). A single, gridded lens located immediately after the first funnel ( $F 1$ in Figure 1.1) serves as the first ion gate (G1 in Figure 1). lons are pulsed into the drift region at G1 (-40 V) for $150 \mu$ s every 20 to 30 ms. Data used to construct ion $t_{D}$ distributions are collected using a time-delayed voltage drop (-3 V) applied to G2. The time delay for opening G2 relative to the initiation pulse at G1 using a step resolution of $0.15 \mathrm{~ms}$ (tryptic digest sample) and 0.2 ms (metabolite extract sample) was scanned across the mobility distribution range. For the tryptic digest this range was $4 \mathrm{~ms}$ to $12 \mathrm{~ms}$ resulting in 41 mobility-resolved mass spector. For the metabolite sample, the range was $3.4 \mathrm{~ms}$ to $15 \mathrm{~ms}$ yielding 59 mobilityresolved mass spectra. The mass spectra are combined to create $2 \mathrm{D} t_{D}(\mathrm{~m} / \mathrm{z})$ datasets (see below). The introduction pulse at G1 and variable delay settings at G2 are controlled by 2 four-channel digital pulse generators (Standford Research Systems, Sunnyvale, CA, USA).

\subsubsection{Parallel Dissociation Methods.}


Parallel dissociation within a drift tube, (IMS-CID-MS, IMS $\left.{ }^{n}-M S\right)$ has been previously described in detail[36, 38] and demonstrated for 'omics applications[7]. Briefly, mobility separated ions are subjected to energizing collisions at the second ion activation region (IA2 in Figure 1.1). For dissociation, the voltage between the last two electrodes of the F2/IA2/G2 assembly (Figure 1.1) is elevated to 220 V over a length of $\sim 0.3 \mathrm{~cm}$. This produces a field of $\sim 700 \mathrm{~V} \cdot \mathrm{cm}^{-1}$ to induce ion fragmentation prior to entering the mass analyzer. Thus low-field settings $\left(\sim 11 \mathrm{~V} \cdot \mathrm{cm}^{-1}\right)$ and high-field settings $\left(\sim 700 \mathrm{~V} \cdot \mathrm{cm}^{-1}\right)$ are employed to transmit precursor and fragment ions, respectively. For neutral loss experiments (see below), more gentle activation conditions $\left(\sim 453 \mathrm{~V} \cdot \mathrm{cm}^{-1}\right)$ are employed in the collisional activation region.

\subsubsection{Mass Spectrometry Measurements}

Mass spectra are generated for all ions by deactivating the dual drift tube gates and setting the mass analyzer scan parameters over a $\mathrm{m} / \mathrm{z}$ range of 80 to 2000 while enabling the automatic gain control (AGC). The AGC threshold is $1 \times 10^{6}$ ions. In this operational mode, the drift tube acts to transmit all ions into the mass analyzer. During $t_{D}$ distribution generation, the gates are activated to transmit a specific mobility into the linear trap. For these analyses, the AGC is disabled and sample injection times of 400 ms (4 microscans) for the tryptic digest and $100 \mathrm{~ms}$ (1 microscan) for the metabolites are employed.

$\mathrm{MS}^{n}$ analysis is conducted by mobility-selecting and isolating $(\mathrm{m} / \mathrm{z})$ a precursor ion. For experiments employing CID in the ion trap, a $\mathrm{m} / \mathrm{z}$ isolation window of $\pm 1.0 \mathrm{Da}$ relative to the centroid $\mathrm{m} / \mathrm{z}$ value results in the ejection of all other ions within the $t_{D}$ window. Selected ions are collisionally activated by a resonant if excitation waveform 
for $10 \mathrm{~ms}$ using a normalized activation energy of $35.0 \%$. Fragment ions displaying sufficient signal-to-noise $(\mathrm{S} / \mathrm{N})$ levels are isolated in the linear ion trap and subsequently fragmented. For experiments employing ETD, a $\mathrm{m} / \mathrm{z}$ isolation window of $\pm 1.0 \mathrm{Da}$ relative to the centroid $\mathrm{m} / \mathrm{z}$ value was utilized. The acquisition time was set at $100 \mathrm{~ms}$. All precursor and fragment ion spectra were recorded using the XCalibur 2.2 software suite (ThermoScientific, San Jose, CA).

\subsubsection{Generating IMS-MS Datasets}

Two-dimensional IMS-MS datasets are generated by converting each $t_{D}$-selected mass spectrum (.RAW file) to a separate text file. Using software developed in house, the $\mathrm{m} / \mathrm{z}$ values and intensities from all of the text files are then associated with the respective $t_{D}$ selection time generating a three-column array text file containing $\left(t_{D}, \mathrm{~m} / \mathrm{z}\right.$, and intensity values). Intensity filters are applied to generate three-column array files of manageable sizes. To generate $t_{D}$ distributions for specific ions (including fragment ions from parallel dissociation), a separate program (in house) integrates all intensities within a user-defined $\mathrm{m} / \mathrm{z}$ range for each $t_{D}$ window. As mentioned above, this is termed a XIDTD.[42]

\subsubsection{Comparing of IMS-MS Datasets.}

IMS-MS $\left[t_{D}(m / z)\right]$ datasets (triplicate measurements) were compared across the four metabolite samples using intensity integration for user-defined pixels over a designated range in the $t_{D}(\mathrm{~m} / \mathrm{z})$ distribution. For these comparisons, each pixel represented $\mathrm{m} / \mathrm{z}$ and $t_{D}$ windows of $2 \mathrm{~m} / \mathrm{z}$ units and $500 \mu \mathrm{s}$, respectively. Data normalization is performed by dividing all integrated pixels in the IMS-MS dataset by the 
total ion intensity. Because of the high-reproducibility of the IMS measurements, alignment of dataset pixels was not performed.

\subsubsection{Detection Limit Sample Preparation}

A model peptide of sequence KKDDDDIIKIIK (Genscript, 90\%) was prepared in 1:1 $18 \mathrm{M} \Omega \mathrm{H} 2 \mathrm{O}$ :methanol containing $1 \%$ formic acid at a stock concentration of $1 \mathrm{mg} \cdot \mathrm{mL}^{-1}$. Using a serial dilution method, a working sample $\left(0.1 \mu \mathrm{g} \cdot \mathrm{mL}^{-1}\right)$ was prepared for sensitivity studies. The working sample was delivered by a syringe pump (KD scientific Holliston, MA, USA) using a 500- $\mu$ L syringe (Hamilton, Reno, NV, USA) to a pulled-tip nano-electrospray emitter $(350 \mu \mathrm{m}$ o.d. $\times 75 \mu \mathrm{m}$ i.d.). The sample flow was maintained at $60 \mathrm{~nL} \cdot \mathrm{min}^{-1}$ and a bias of $1.7 \mathrm{kV}$ was used during direct infusion of the sample solution.

\subsubsection{Dynamic Range Studies}

A phosphopeptide standard (Anaspec, Fenton, CA) having a sequence of SFVLNPTNIGMsKSSQGHVTK (M.W. 2316.6) was added to the trypic digest solution at a concentration of $0.01 \mu \mathrm{g} \cdot \mu \mathrm{L}^{-1}$. The standard was chosen due to efficient ionization properties. During IMS-MS dataset generation of the tryptic digest, the [SFVLNPTNIGMsKSSQGHVTK +3$]^{3+}$ ion $(\mathrm{m} / \mathrm{z} 771.9)$ was evaluated (XIDTD) for maximum intensity because it produced the largest signal. In addition to this ion, the signal level for the low-intensity $[\mathrm{KYIPGTK}+1 \mathrm{H}]^{1+}$ peptide ion from the cytochrome $\mathrm{c}$ digest was also evaluated from a XIDTD.

\subsubsection{Principal Component Analysis (PCA)}

Using the JMP statistical software package (JMP, Version 7. SAS Institute Inc., Cary, NC, 1989-2007) PCA has been performed for the triplicate analyses of each 
cohort. For PCA, inputs included the normalized pixel intensities (see above). A comparison of dataset features providing the greatest contribution to distinguishing the datasets by PCA is performed using the loadings matrix (LM) values for all pixel inputs. High LM scores ( $\geq 0.90$ in absolute value) along principal component one (PC 1) result in the determination of pixels for which dataset features exhibit large differences in intensity.

\subsubsection{Exact Mass Measurements.}

A Q-Exactive Orbitrap mass spectrometer (Thermo Scientific, San Jose, CA, USA) has been used for exact mass measurements. Briefly, the EXP24 sample is introduced by direct infusion through a heated electrospary source inlet (HESI) using a $2.5 \mathrm{kV}$ spray voltage bias relative to the entrance orifice and a capillary temperature of $250{ }^{\circ} \mathrm{C}$. The generated ions pass through the S-lens ion guide $(60.0 \mathrm{~V})$ and are subsequently transferred into an Orbitrap mass analyzer. The Orbitrap is scanned from $\mathrm{m} / \mathrm{z} 100.0$ to 1000.0 with a resolving power of 140,000 . A target AGC of $1.0 \times 10^{6}$ with a $200 \mathrm{~ms}$ injection time has been used for the analysis.

\subsection{Results and Discussion}

\subsubsection{Instrument Figures of Merit}

Instrument figures of merit. A number of the measurement capabilities of the instrument are here reported to provide context for its utility in complex mixture analysis. The resolving power $\left(t_{D} / \Delta t_{D} \mathrm{FWHM}\right)$ capabilities of the instrument can be provided from the XIDTDs of a number of singly-charged peptide ions. The singly-charged ions are used because they are observed in a less congested area of the two-dimensional (2D) IMS-MS dataset and therefore are less influenced by overlapping ions. For the peptides 
$[\mathrm{MIFAGIK}+\mathrm{H}]^{+},[\mathrm{YIPGTK}+\mathrm{H}]^{+}$, and $[\mathrm{GITWK}+\mathrm{H}]^{+}$resolving power values of 36,46 , and 40 have been computed. It is noted that for this $1-\mathrm{m}$ long drift tube, the resolving power is somewhat limited by the gate width of the second ion gate ( $G 2$ in Figure 1.1)Because of the finite distance $(3 \mathrm{~mm})$ of the Tyndall gate, a relatively large "open" time is required to transmit the ions of interest. This will limit the achievable resolving power. Increasing the drift tube length to $2 \mathrm{~m}$ and $3 \mathrm{~m}$ would lessen the effect of this drift time $\left(t_{D}\right)$ bin size.

\subsubsection{Detection Limit Studies}

The detection limits of the instrument have been determined for individual peptide ions. This is accomplished by serial dilution of a given peptide in ESI solution until ion signals are just perceptible for mobility-selected species. Figure 2.1 shows the mass spectral peak for mobility-selected $[\mathrm{M}+3 \mathrm{H}]^{3+}$ ions of the model peptide KKDDDDDIIKIIK. Here, the concentration of the peptide was $0.1 \mu \mathrm{g} \cdot \mathrm{mL}-1$. The spectrum represents a 1.0 second signal averaging from a 6 second data acquisition. The signal-to-noise ratio $(\mathrm{S} / \mathrm{N})$ of 31 suggests that detection limits $(\mathrm{S} / \mathrm{N}=3)$ for this peptide are 64 attomoles. Based on signal levels for other peptides, it is estimated that the detection limits for mobility-selected ions (dominant charge states) are generally tens of attomoles. 


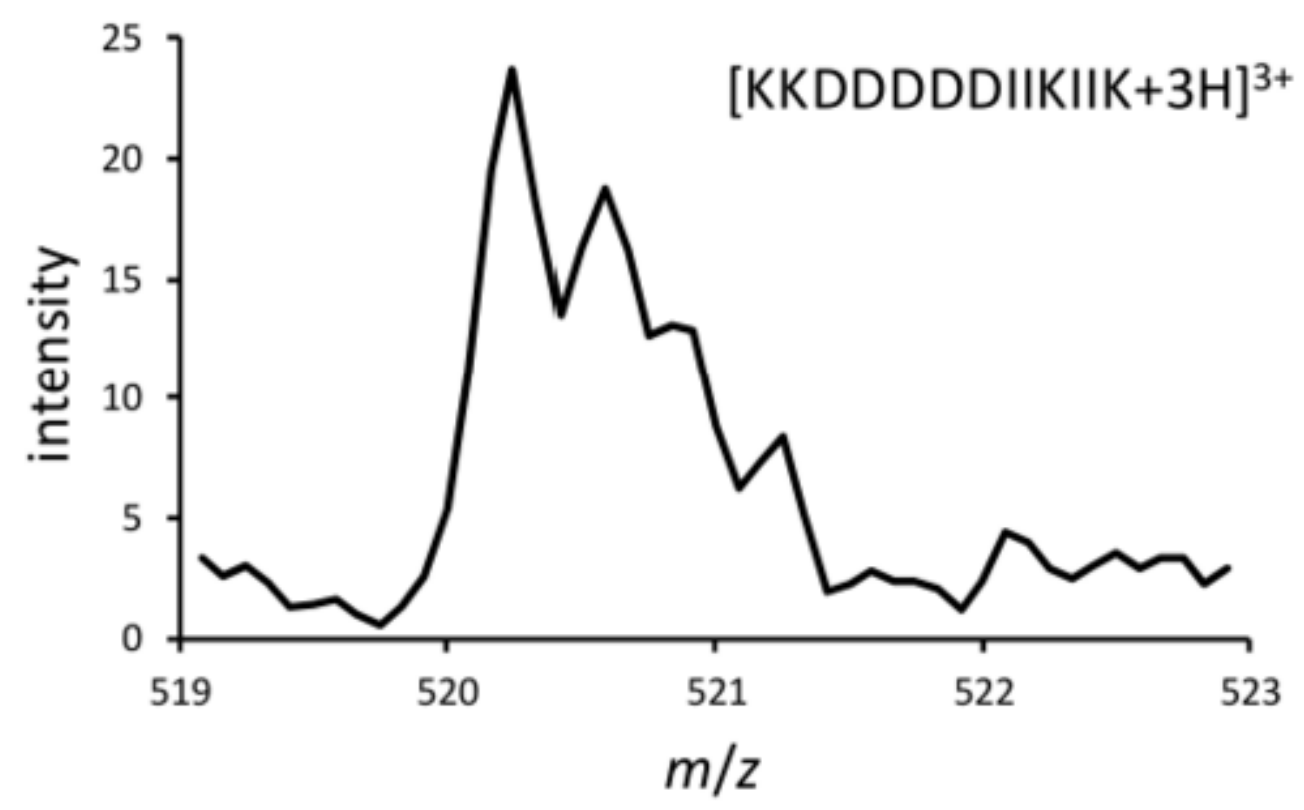

Figure 2.1. Mass spectrum showing the $[\mathrm{M}+3 \mathrm{H}]^{3+}$ ions of the KKDDDDDIIKIIK model peptide. Parameters for spectrum collection included a $1000 \mathrm{~ms}$ trap injection time. The ion current was averaged over 1 second.

\subsubsection{Dynamic Range Studies}

The dynamic range of the instrument can be estimated by comparisons of peak intensities from IMS-MS datasets. An example comparison of XIDTDs for two different ions is shown in Figure 2.2. Only peptides having a $\mathrm{m} / \mathrm{z}$ that could be used for identification were used for dynamic range determination. Figure 2.2A shows the XIDTD for the cytochrome $\mathrm{c}$ tryptic peptide, $[\mathrm{KYIPGTK}+1 \mathrm{H}]^{+}$having $\mathrm{m} / \mathrm{z}$ 806.5. The lowintensity feature at $t_{D} \sim 11.2 \mathrm{~ms}$ is observed to fall within the singly-charged trend line shown in Figure 2.3A (see below). The integrated intensity for $m / z 806.5$ is $\sim 37$. Figure 2.2B shows data for a phosphorylated peptide standard having a $\mathrm{m} / \mathrm{z} 771.9$. The XIDTD shows a dominant conformation at $7.4 \mathrm{~ms}$ followed by unresolved larger conformations. Here, the integrated area is 43764 . These numbers demonstrate at dynamic range of $>1.0 \times 10^{3}$. Here we note that a higher dynamic range is observed when using lowersignal species (integrated intensities of $\sim 5$ counts - above the $S / N$ threshold of 3 ). Such 
ions cannot be identified as cytochrome $\mathrm{c}$ digest ions however, and therefore the dynamic range is presented as $>10^{4}$.
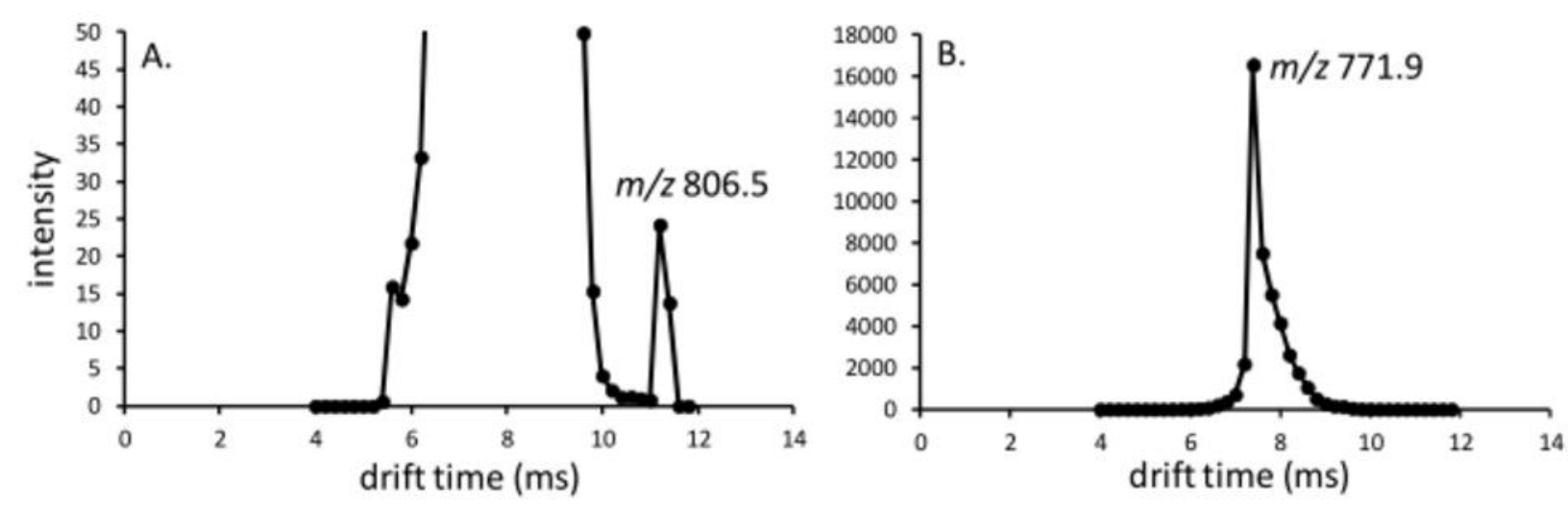

Figure 2.2. (A) XIDTD of the cyochrome $c$ tryptic peptide ion, [KYPIGTK+H] ${ }^{+}$, of $m / z 806.5$. That dataset feature corresponding to these ions is centered at $t_{D}=11.2 \mathrm{~ms}$. (B) XIDTD of the [SFVLNPTNIGMsKSSQGHVTK $+3 \mathrm{H}]^{3+}$ ions of $\mathrm{m} / z$ 771.9. The entire $t_{D}$ distribution corresponds to these ions

\subsubsection{Phosphopeptide Analysis}

Figure 2.3A shows a heat plot of the $2 \mathrm{D} t_{D}(\mathrm{~m} / \mathrm{z})$ dataset of the cytochrome $c$ digest containing the phosphorylated peptide standards. Tryptic peptide ions are observed to exist predominately as $[\mathrm{M}+2 \mathrm{H}]^{2+}$ and $[\mathrm{M}+3 \mathrm{H}]^{3+}$ ions exhibiting similar $t_{D}(\mathrm{~m} / \mathrm{z})$ trends as reported previously.[1] Most spectral features appear between $\sim 5 \mathrm{~ms}$ to $\sim 9 \mathrm{~ms}$ and are observed within the 400 to $1000 \mathrm{~m} / \mathrm{z}$ range. The data in Figure 2.3A represents a number of ions exhibiting an intensity range of $10^{3}$.

The highly-tunable nature of the IMS-CID process $[36,37]$ can be combined with the efficient mobility separations $[64,65]$ to characterize phosphorylated peptides in tryptic digests. Because the bond dissociation energy of the phosphate modification is lower than that of backbone amide fragmentation, an IMS-CID approach that employs gentle activation $\left(\sim 450 \mathrm{~V} \cdot \mathrm{cm}^{-1}\right)$ can be utilized. Here a goal is to increase the activation 
voltage in IA2 (Figure 1.1) until neutral loss $\left(\mathrm{H}_{3} \mathrm{PO}_{4}\right)$ is observed while preserving much of the other tryptic digest peptide ions. This allows for a more straightforward analysis of precursor and product ions similar to ion trap experiments described previously.[40, 41]

\subsubsection{Aligning Extracted lon Drift Time Distributions (XIDTDs)}

For matching the precursor and neutral loss fragment ions, it is useful to evaluate

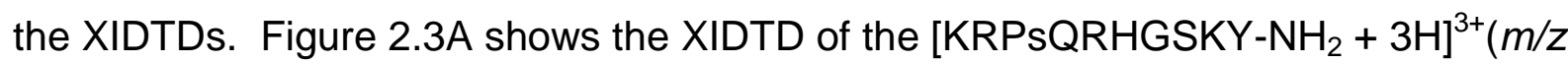
475.2) ions as an inset. This peptide exhibits a broad mobility profile containing more compact $\left(t_{D}=5.2 \mathrm{~ms}\right)$ and more elongated $\left(t_{D}=6.4 \mathrm{~ms}\right)$ species. Figure 2.3B shows $t_{D}(\mathrm{~m} / \mathrm{z})$ data generated from IMS-CID of the digest mixture. The $\mathrm{m} / \mathrm{z}$ difference between the precursor ion and the neutral loss product ion is 32.6 , which is consistent with the loss of $\mathrm{H}_{3} \mathrm{PO}_{4}$ for the triply-charged ion. The XIDTD of the neutral loss product ion is shown as an inset in Figure 2.3B revealing a slightly broader distribution with conformational maxima matching the precursor XIDTD profile (5.2 $\mathrm{ms}$ and $6.4 \mathrm{~ms})$. A difference in the distributions is the higher intensities observed for more elongated species in the IMS-CID dataset. That said, the $t_{D}$ matching of the dominant features in the XIDTDs helps confirm the origin for much of the ions. The differences in the distributions may arise from the presence of overlapping lower-intensity fragment ions.

\subsubsection{IMS - CID - ETD - MS of Precursor and Fragment ions}

Both the intact precursor and neutral loss ions are present in the same $t_{D}(\mathrm{~m} / \mathrm{z})$ distribution (Figure 2.3) and either can be mobility-selected and $\mathrm{m} / \mathrm{z}$ isolated for further characterization by ETD. Figure 2.4A and B show the ETD spectra for the intact precursor and neutral loss fragment ions, respectively. Here the $z$-ion series is most informative for observing the site of phosphorylation. That is, for the precursor ETD 

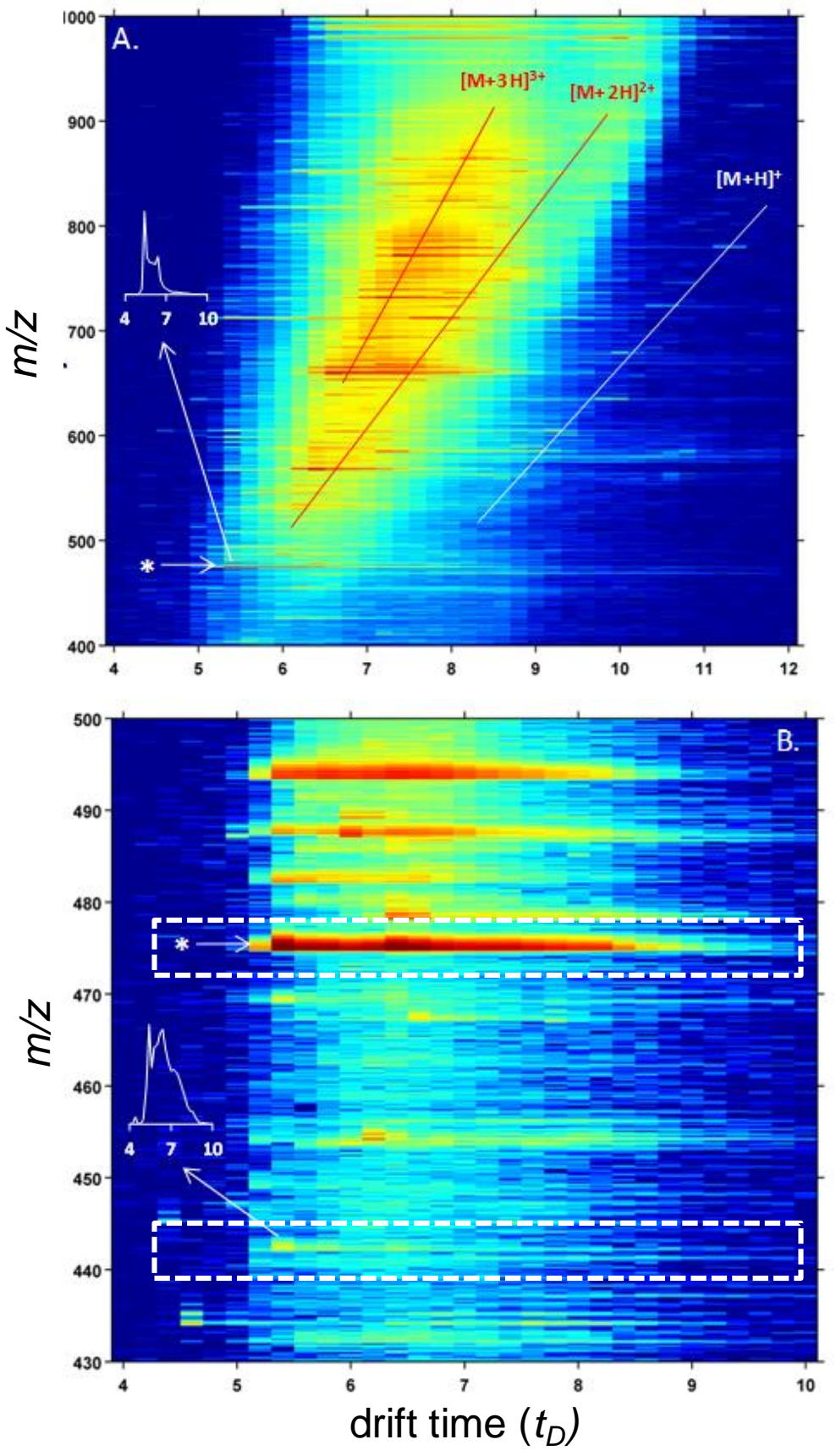

Figure 2.3 (A) Two-dimensional (2D) $t_{D}(\mathrm{~m} / \mathrm{z})$ heat-plot of a cytochrome $c$ tryptic digest containing the phosphorylated peptide standard [KRPsQRHGSKY-NH ${ }_{2}(\mathrm{~m} / z$ 475.2)]. The color map for the plot is shown on a logarithmic scale ranging from -2 to 3 in order to show the low-intensity $[\mathrm{M}+\mathrm{H}]^{+}$ ions. (B) Expanded region of a 2D $t_{D}(\mathrm{~m} / \mathrm{z})$ heat-plot for the same digest sample upon performing IMS-CID. The same color scale is used. The XIDTDs of the precursor and neutral loss product ions are shown as insets on the respective $2 \mathrm{D}$ plots. An asterisk denotes the location of the precursor ion both 2D plots. 
fragments, the $z_{8}$ ion at $\mathrm{m} / \mathrm{z} 1025.4$ reveals residue-specific phosphorylation.

Comparatively, in the neutral loss product ion spectrum, the same $z_{8}$ ion has a $\mathrm{m} / \mathrm{z}$ value of 946.4 . As expected, lower $z$-series ions for both the precursor and neutral loss ions demonstrate the same $\mathrm{m} / \mathrm{z}$ values. For larger ions ( $>z_{8}$ ion), those originating from the precursor species are higher in $\mathrm{m} / \mathrm{z}$ due to the addition of the phosphate moiety.

Also noticeable in the spectra are the ETD charge-reduced species that further confirm the neutral loss product ion. In the case of the $[\mathrm{M}+3 \mathrm{H}]^{2+\bullet}$ ions, the precursor $m / z$ is 712.4 , while the product ion $\mathrm{m} / \mathrm{z}$ is 663.4 . The $\mathrm{m} / \mathrm{z}$ difference (49) is consistent with the neutral loss of $\mathrm{H}_{3} \mathrm{PO}_{4}$ for the doubly-charged ion.

$\left[\text { KRPsQRHGSKY-NH }{ }_{2}+3 \mathrm{H}\right]^{3+}(m / z$ 475.2)

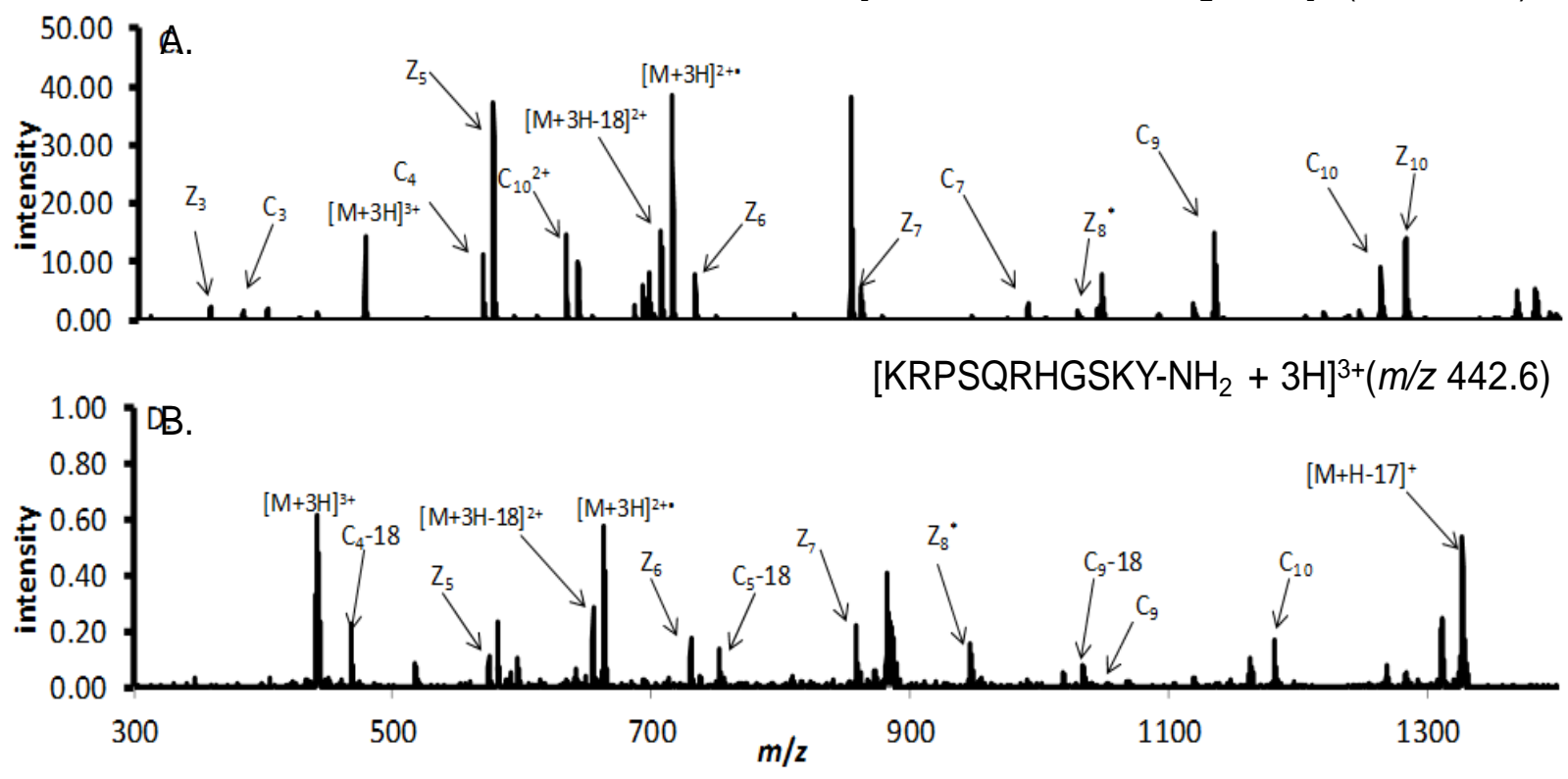

Figure 2.4 (A) and (B) Mobility-selected ETD spectra of the precursor and neutral loss ions, respectively. Identified fragment ions are labeled. Several identified $c$ - and $z$-ions are are labeled as well as the charged reduced molecular species. The $z_{8}{ }^{*}$ fragment ion denotes the neutral loss of $\mathrm{H}_{3} \mathrm{PO}_{4}$ by IMS-CID. This fragment ion corresponds with the $\mathrm{S}^{4}$ residue for the model phosphopeptide. 
The use of ion trapping instruments without ETD capabilities often requires at least $\mathrm{MS}^{3}$ to determine the site of phosphorylation. $[66,67]$ As a result, the technique requires relatively higher ion signal levels for informative higher-order $\mathrm{MS}^{\mathrm{n}}$ results. The use of IMS-CID-ETD-MS for PTM peptide precursor scans is unique in that neutral loss ions can be uniquely matched back to the precursor based on XIDTDs; this can be followed by ETD-MS of the intact precursor for site specific PTM determination. Such a technique also represents an enhanced scanning approach compared to that employing CID and ETD in an ion trap[40, 41] as the mobility matching and selection would help to identify precursor ions and improve their isolation for PTM site determination.

It is also noted that the IMS-CID technique is data independent. That, is fragmentation of the precursor ions are signal dependent to trigger CID. Instead, IA2 is used to preferentially fragment the phosphodiester bond. In turn any produced fragment ions are matched based on XIDTDs for ETD-MS analysis. This further differentiates the technique from other MS/MS methods.

\subsubsection{A Comparative IMS - MS Analysis of Metabolimic Samples}

2D IMS-MS datasets have been recorded in triplicate for the four metabolite extract samples. Figure 2.5, shows the $t_{D}(\mathrm{~m} / \mathrm{z})$ heat plot of metabolites obtained from a single IMS-MS analysis of the EXP24 pooled sample. For all datasets, spectral features are first observed at $\sim 3.4 \mathrm{~ms}$ and extend to $\sim 15.0 \mathrm{~ms}$; the highest intensity metabolite features exist between $\sim 8.0 \mathrm{~ms}$ to $\sim 9.0 \mathrm{~ms}$. In general, dataset features are observed over a wide $\mathrm{m} / \mathrm{z}$ range from $\sim 80$ to $\sim 1200$ with the ions exhibiting the greatest intensity being observed over a $\mathrm{m} / \mathrm{z}$ range of $\sim 500$ to $\sim 650$. Using the pixel integration sizes 
mentioned above, dataset pixel intensities for 19,000 separate $t_{D}(\mathrm{~m} / \mathrm{z})$ regions range from 0 to $>10^{4}$.

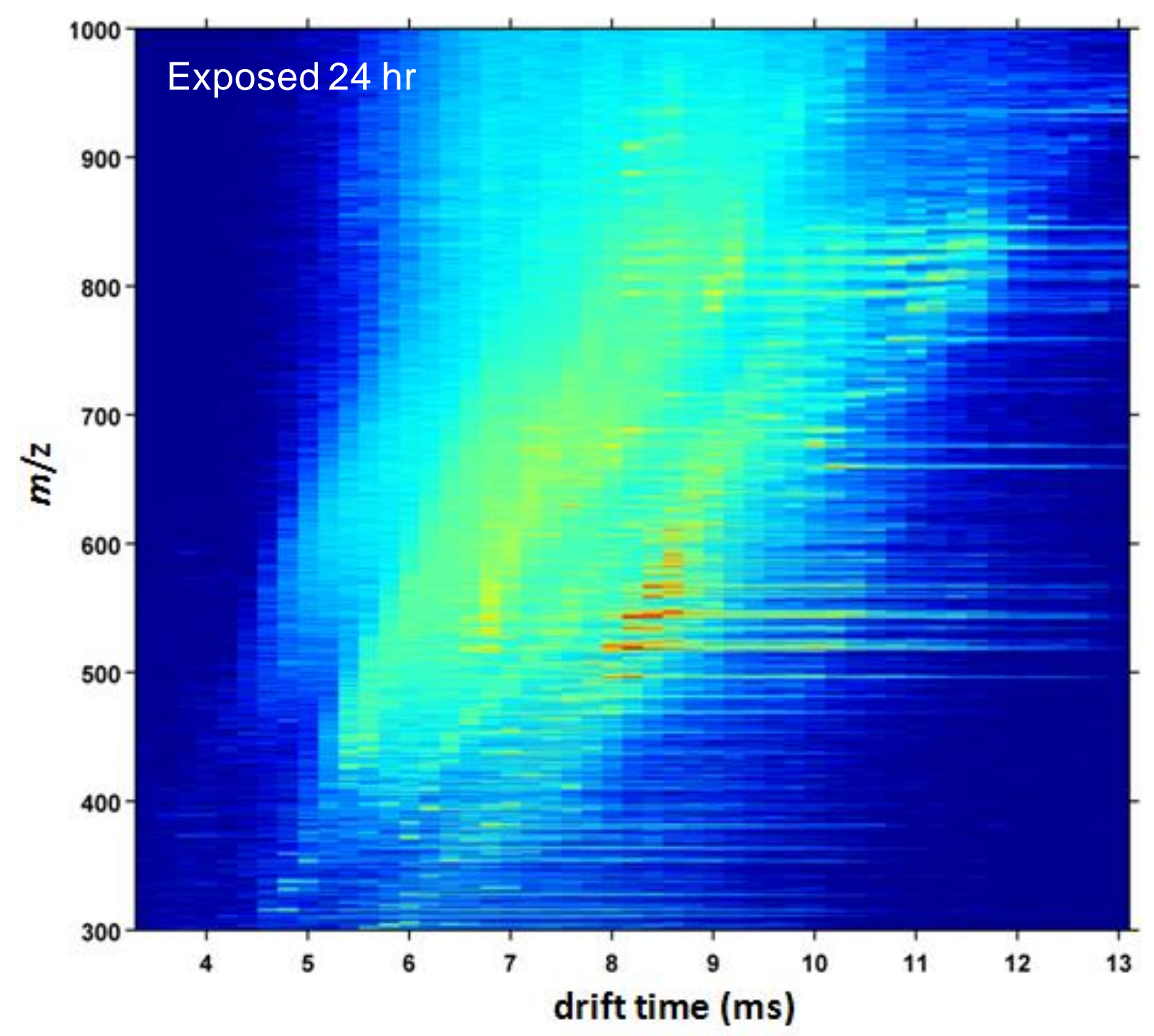

Figure 2.5 (A) 2D $t_{D}(\mathrm{~m} / \mathrm{z})$ heat-plot of one replicate analysis of the EXP24 metabolite sample. The color map for the plot is shown on a logarithmic scale ranging from -2 to 3 in order to show low-intensity features.

\subsubsection{PCA of Sample Cohorts}

The two-dimensional PCA plot obtained from triplicate measurements of the samples is shown in Figure 2B. Overall PC 1 contributes the greatest to the differentiation of all samples, with only a small degree of variation along PC 2. Data 
points for triplicate measurements for both CNTRL0 and CNTRL24 are observed to cluster more toward the center of the PCA plot (Figure 2.6A). Comparatively, points for the EXP24 and EXP0 samples show a slightly higher degree of variation associated with PC 1. Therefore, the separation along PC 1, to a greater degree, is related to differences in dataset features associated with organism post exposure time. PC 1 then becomes a factor for focusing the analysis to a select number of spectral features (see below). It is worth noting that, compared to triplicate LC-MS experiments (data not shown), replicate dataset similarity is increased for the IMS-MS datasets.

Dataset features with the greatest contributions to distinguishing the pooled samples via PCA can be identified from the loadings matrix (LM) as shown in Figure 2.6B. Comparing data recorded for the EXP24 and EXP0 samples, LM values that exhibit high positive $\mathrm{x}$-values correspond to a direct correlation for the EXP0 sample; that is, these $t_{D}(\mathrm{~m} / \mathrm{z})$ regions exhibit higher intensities, in general, for this sample. Conversely, the pixel intensities associated with the largest negative LM values correspond to an inverse correlation for the EXPO samples. The absolute value of LM scores for dataset features exhibiting $\mathrm{m} / \mathrm{z}$ values of $542.3\left(t_{D}=7.4 \mathrm{~ms}\right)$ and $518.3\left(t_{D}=\right.$ $7.4 \mathrm{~ms})$ are 0.98 and 0.97 respectively, representing the greatest contributions to the PCA. These dataset features as well as those for several other metabolites having LM values $\geq 0.90$ in magnitude are considered for further analysis and tentative metabolite identification. Although somewhat arbitrary, this threshold provides a stringent filter for focusing the analysis on a select few dataset features (35) from hundreds of potential candidates. 
The dataset features along with tentative assignments obtained from accurate mass matching and precursor ion fragmentation are listed in Table 2.1.
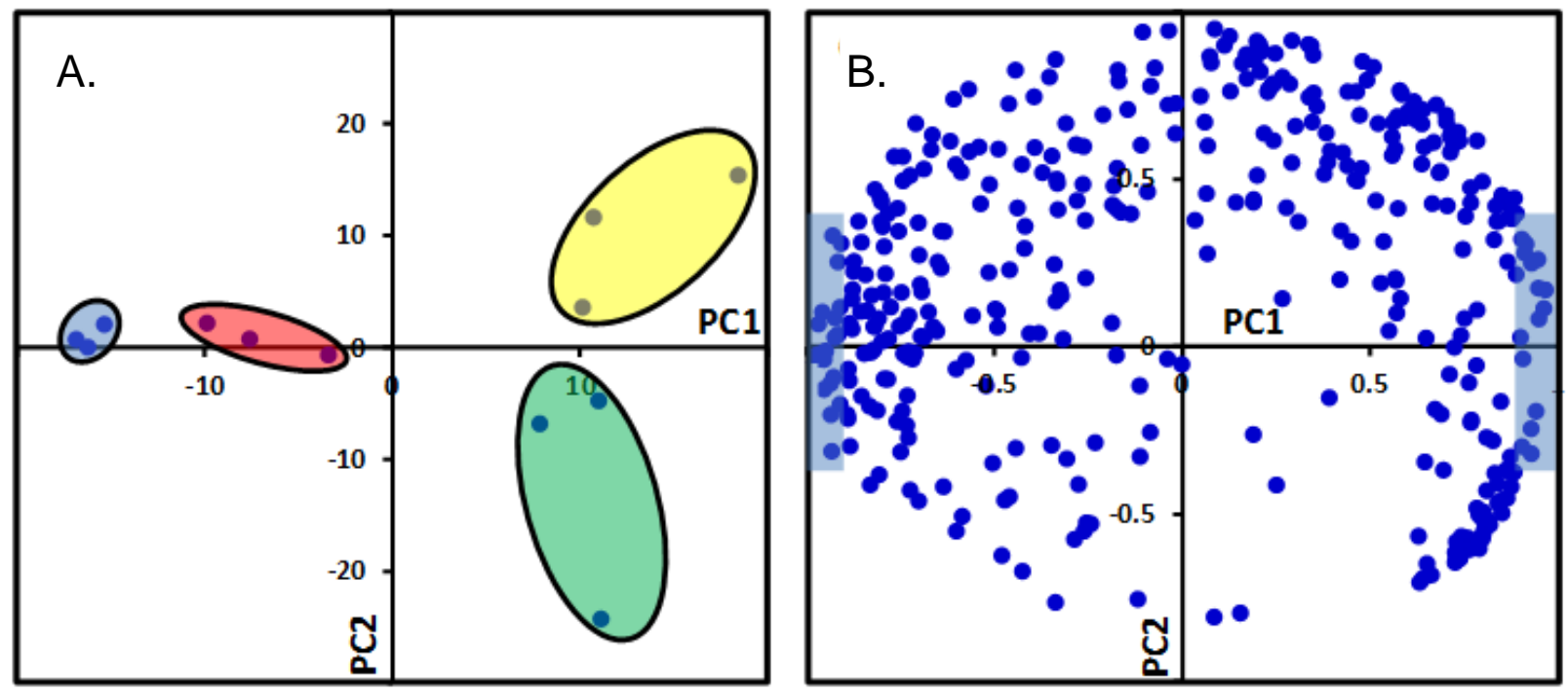

Figure 2.6(A) PCA of the triplicate measurements for all sample cohorts including, Blue: CNTRL24: Red: CNTRL0; Yellow: EXP24; Green: EXP24. The PCA plot was generating using normalized intensities for $t_{D}(\mathrm{~m} / \mathrm{z})$ pixels from the IMS-MS datasets. (B) Loadings matrix (LM) values showing the degree of contribution to PCA separation by specific pixels across all sample cohorts along PC 1 and 2. Shaded areas represent those having $\mid L M$ value $\mid \geq 0.90$.

\subsubsection{Comparing Extraction Ion Drift Time Distributions (XIDTDs)}

Rapid comparisons of percent differences in pixel intensity across multiple datasets can reveal features that are significantly different even when they are of much lower intensity. Figure 2.7A and B shows the XIDTDs of ions of $\mathrm{m} / z 518.3$ for the EXP24 and CNTRL24samples. Dataset replicates of the EXP24 sample show the 


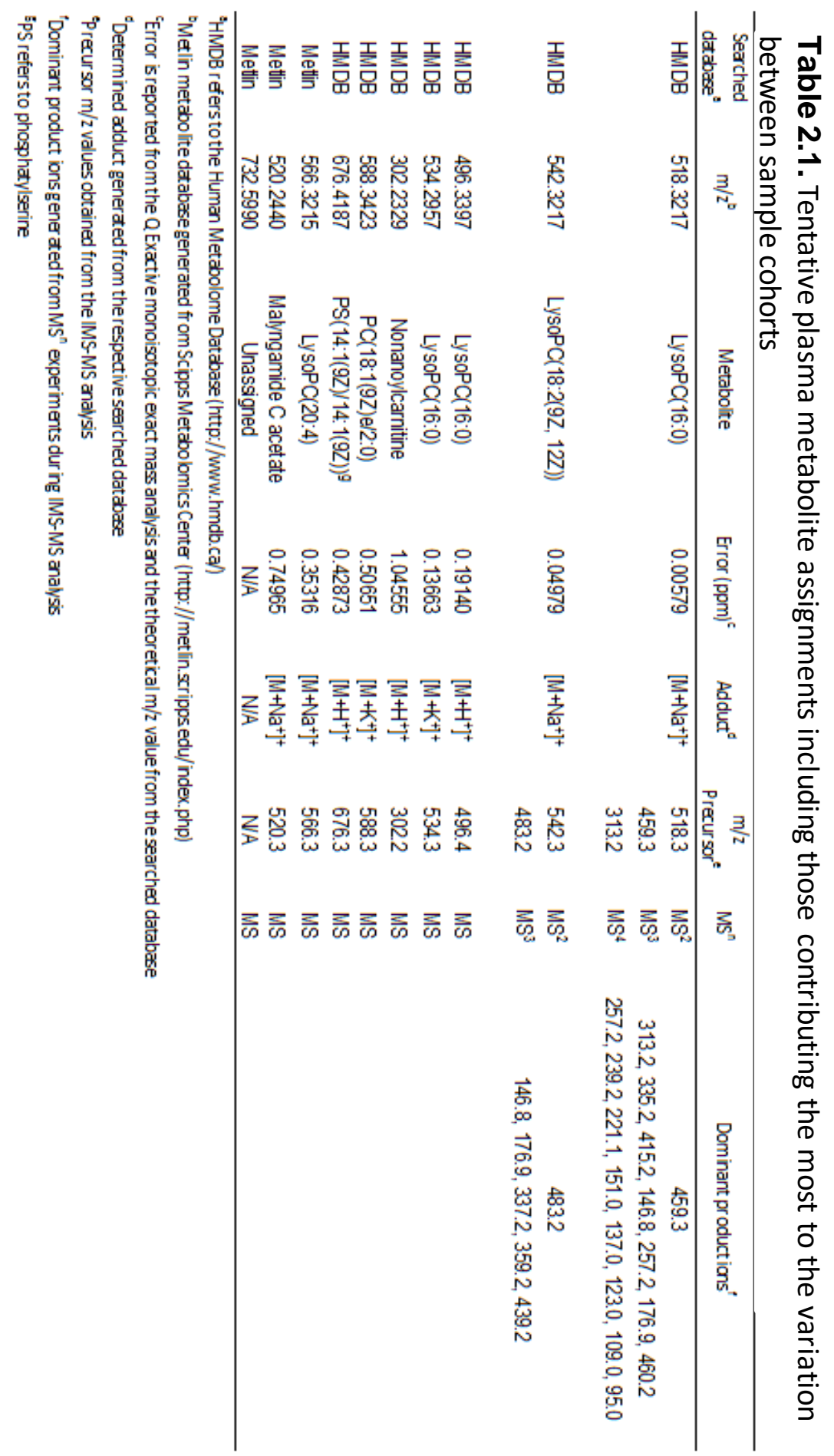



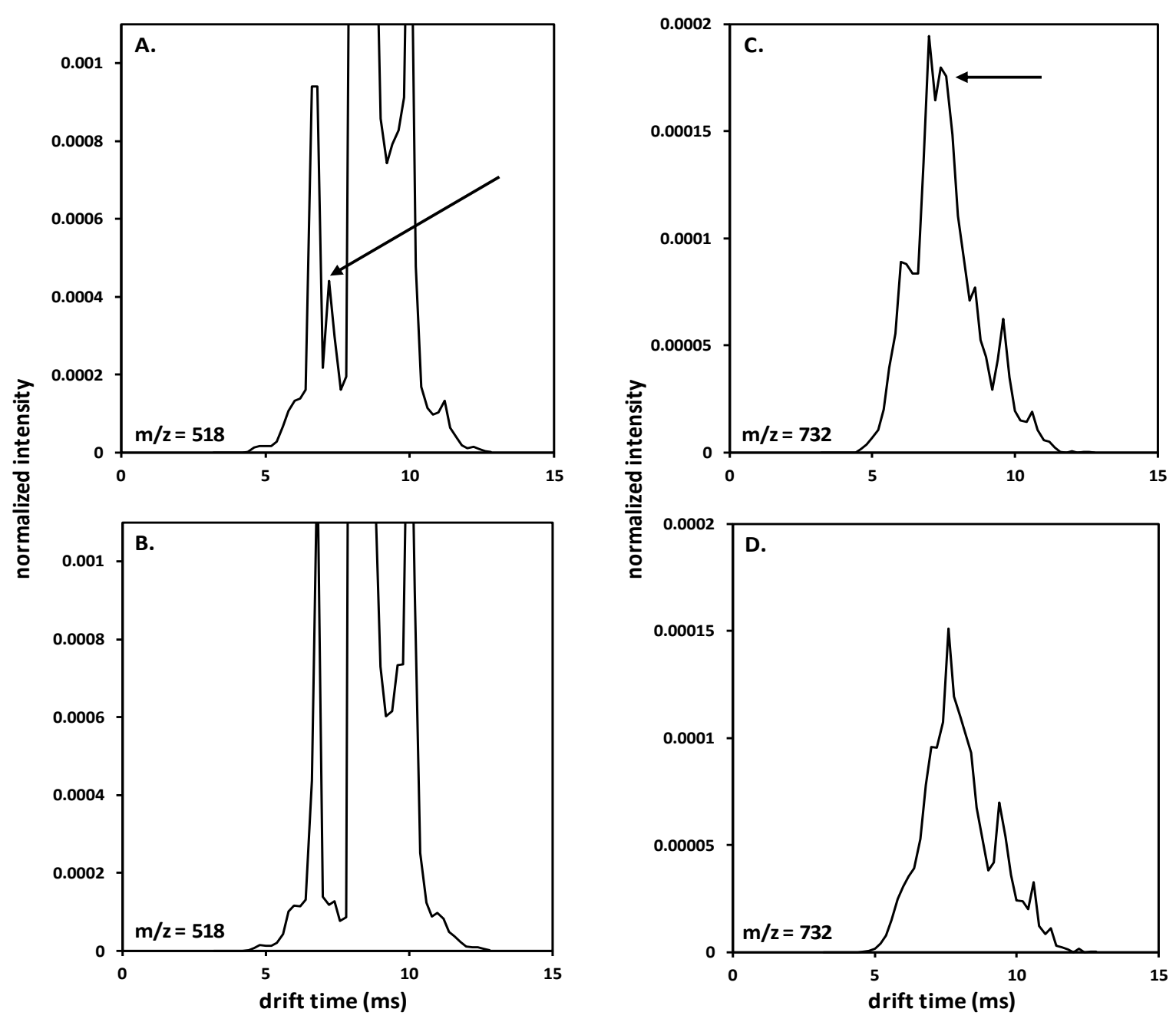

Figure 2.7 (A) Extracted ion drift time distribution (XIDTD) of the ion of $\mathrm{m} / \mathrm{z} 518.3$ for the CNTRL24 sample (single replicate). (B) XIDTD of the ion of $\mathrm{m} / z 518.3$ for the EXP24 sample (single replicate). The XIDTD is obtained by integrating all intensities over $\mathrm{m} / \mathrm{z}$ range of 6 (centered about the average $\mathrm{m} / \mathrm{z}$ of the ion) across all drift times. (C) XIDTD for $\mathrm{m} / \mathrm{z} 732.2$ for EXP24 and (D) CNTRL 24, respectively. Each distribution is normalized such that the total area under the curve is unity. The arrow indicates a difference in spectral features between the two samples that have been recognized originally in the pixel-by-pixel intensity comparison of the

presence of a higher abundance feature at $\sim 7.2 \mathrm{~ms}$. The average normalized pixel intensities for this region in the EXP24 replicates is $0.64 \pm 0.02$ compared to $0.11 \pm 0.01$ for the CNTRL24 replicates. Similar spectral comparisons can be made for other ions. Figure 2.7C and $D$ show that for $m / z 732.2$, several differences are observed. For example the peak intensities for ions with $t_{D} \sim 7.4 \mathrm{~ms}$ and $\sim 7.6 \mathrm{~ms}$ are greater for EXP 
24 relative to the CNTRL 24 sample cohorts. Although the features in the XIDTD cannot be ascribed to different gas-phase conformers or isobaric ions, Figure 2.7 shows the ability to rapidly compare distinguishing dataset features that may otherwise not be considered to be significantly different between samples by MS analysis alone.

Additionally, the comparison reveals the high-reproducibility of the measurement.

Without having to employ peak (pixel) alignment techniques, the coefficients of variation for the low-intensity $\mathrm{m} / \mathrm{z} 518.3$ feature are $~ 3 \%$ (EXP24 replicates) and $~ 9 \%$ (CNTRL24 replicates).

\subsubsection{IMS - MS Experiments for Metabolomic Analysis}

The low-pressure drift tube allows trapping of electrosprayed ions increasing the overall sensitivity and enabling $\mathrm{MS}^{\mathrm{n}}$ experiments for mobility-selected ions. To demonstrate this capability, metabolite ions exhibiting a $t_{D}$ of $\sim 8.2 \mathrm{~ms}$ and having $\mathrm{m} / \mathrm{z}$ 518.3 have been selected for $\mathrm{MS}^{\mathrm{n}}$ analysis. A precursor mass search of the Human Metabolome Database (HMDB)[68] presented the sodiated lysophosphatidylcholines LysoPC(16:0) as the top match. $\mathrm{MS}^{2}$ of this ion yields essentially one fragment ion at $\mathrm{m} / \mathrm{z} 459.2$ as shown in Figure 2.8A. This ion could indicate the loss of a methylated ammonium head group $(m / z 59)$ that is characteristic to many LysoPC.[69, 70] Fragments that displayed the highest $\mathrm{S} / \mathrm{N}$ ratios have been used in subsequent fragmentation experiments. For example, activation of the $m / z 459.2$ ion produced from the mobility-selected $\mathrm{m} / \mathrm{z} 518.3$ precursor yields several peaks including those at $\mathrm{m} / \mathrm{z}$ 

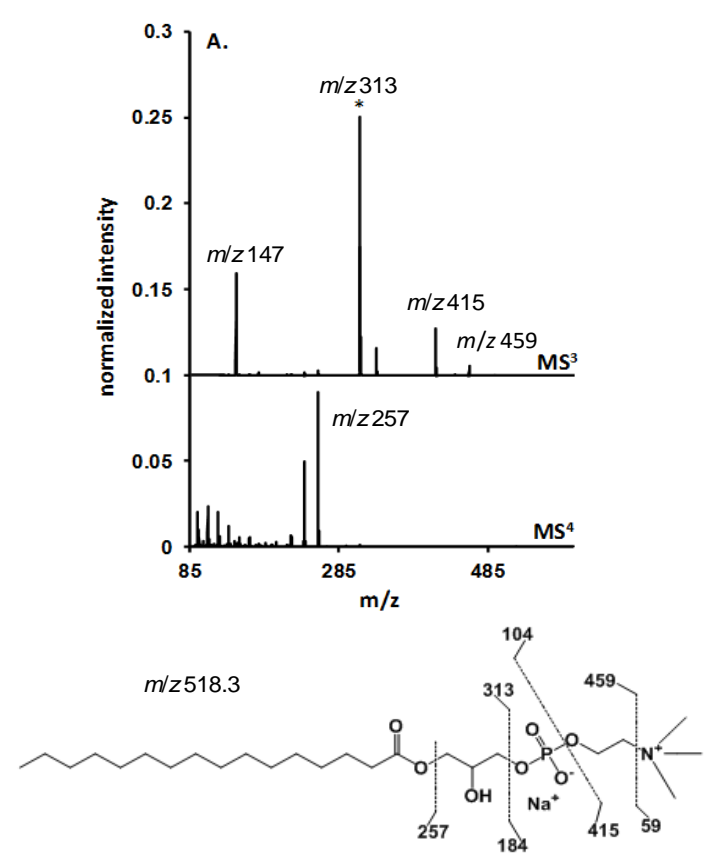
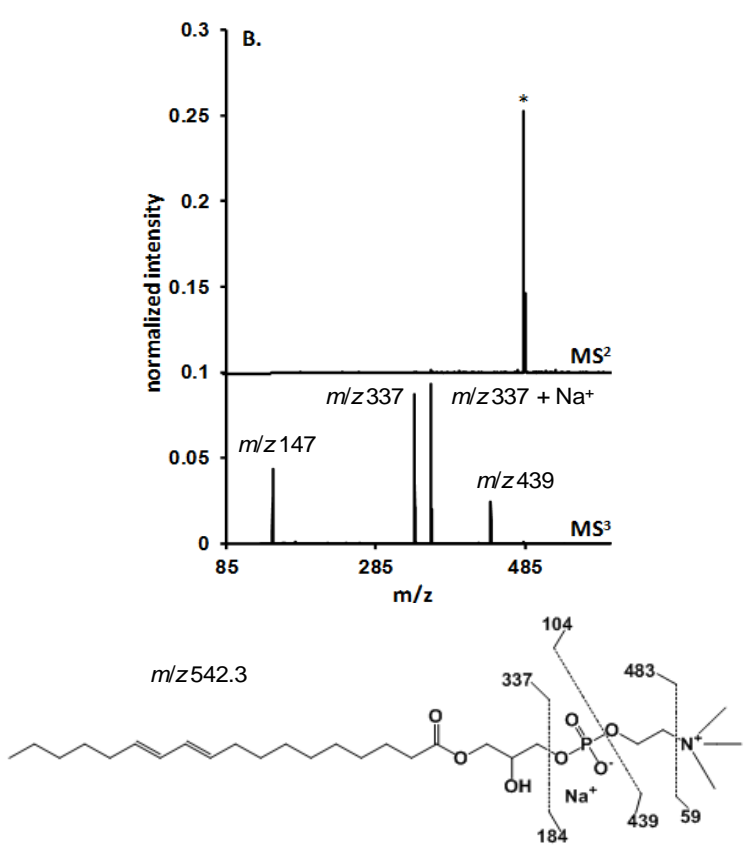

Figure 2.8 (A) Multistage tandem mass spectrometry $\left(\mathrm{MS}^{\mathrm{n}}\right)$ of the drift selected $\left(t_{D} 8.2 \mathrm{~ms}\right)$ precursor ion of $m / z 518$.3. The top spectrum shows the $\mathrm{MS}^{3}$ spectrum obtained upon activation of the major product ion $(\mathrm{m} / \mathrm{z}$ 459.2) generated during MS/MS (spectrum not shown). The bottom spectrum shows $\mathrm{MS}^{4}$ fragments produced by selecting the ion of $m / z 313.2$ from $\mathrm{MS}^{3}$ experiments. (B) $M S^{n}$ of the drift selected $\left(t_{D} 8.2 \mathrm{~ms}\right.$ ) precursor ion $\mathrm{m} / z$ 542.3. The top panel shows the MS/MS spectrum resulting in the dominate fragment ion $\mathrm{m} / \mathrm{z}$ 483.2. The bottom spectrum shows the $\mathrm{MS}^{3}$ upon collsionally activated of $\mathrm{m} / \mathrm{z}$ 483.2. Several identified fragment ions are labeled in each respective panel and referenced back to the tentative structures (below respective spetrum. The peak marked with an asterisk indicates the $\mathrm{m} / \mathrm{z}$ selected for subsequent MS analysis.

313.2, 335.2, and 415.2. Isolation of $\mathrm{m} / z 313.2$ ions allows for $\mathrm{MS}^{4}$ analysis that produces several features. Although not possible to assign all fragment ions, the peak at $\mathrm{m} / \mathrm{z} 257.2$ is consistent with the alkyl chain and ester region of a LysoPC molecule. When compared to the feature at $m / z 257.2$, the product ion with $\mathrm{m} / \mathrm{z} 239.2$ indicates a loss of water. Additionally, a number of low $\mathrm{m} / \mathrm{z}$ fragments differing by $14 \mathrm{Da}$ is consistent with fragmentation of the aliphatic chain of a LysoPC molecule. The inset in Figure $2.8 \mathrm{~A}$ shows a schematic representation of the sodiated LysoPC(16:0) molecule and the observed fragments that are consistent with those obtained from the precursor ion of $m / z 518.3$. 
Similar fragmentation is also observed for the mobility selected ion of $\mathrm{m} / \mathrm{z}$ 542.3. Figure 2.8B shows the $\mathrm{MS}^{2}$ spectrum from $\mathrm{m} / \mathrm{z} 542.3$ where a dominate ion of $\mathrm{m} / \mathrm{z} 483.2$ was observed. This fragment ion is indicative of the choline head region $(\mathrm{m} / \mathrm{z}$ 59). For the $\mathrm{MS}^{3}$ scan, the fragment ion of $\mathrm{m} / \mathrm{z} 483.2$ was selected and collisionally activated. Several fragments ions are observed and are consistent with a LysoPC. For example, $\mathrm{m} / \mathrm{z} 125.3$ is consistent with the loss of the phosphate region minus to the $\mathrm{MS}^{2}$ loss of the choline. The fragment ion $\mathrm{m} / \mathrm{z} 337.1$ may indicate that the alkyl chain may contain two alkene regions. However, the exact locations of these regions were not capable of being identified do to signal restraints.

The tentative molecular assignment obtained from the $\mathrm{MS}^{4}$ and $\mathrm{MS}^{3}$ spectra from both ion experiments is further supported by accurate mass matches $( \pm 0.5 \mathrm{ppm})$ obtained by direct electrospray of the sample into a Q-Exactive Orbitrap (ThermoScientific) mass spectrometer. Such assignments are presented as tentative as biomarker discovery efforts would require exact mass and ion fragmentation matches to molecular standards. That said, the IMS-MS instrumental approach is presented here as a means to rapidly provide candidate molecules for which confirmatory experiments can be conducted.

\subsubsection{IMS - CID - MS Measurements}

Not requiring user defined isolation and fragmentation of each individual ion, shotgun proteomics experiments have demonstrated that parallel dissociation[43] of ions in a drift tube offers significant advantages in terms of speed.[7, 71] Such a speed increase using the current IMS-MS instrument can be advantageous for rapid comparative metabolomics analyses as well. Figure 2.9A shows a 2D IMS-CID-MS 

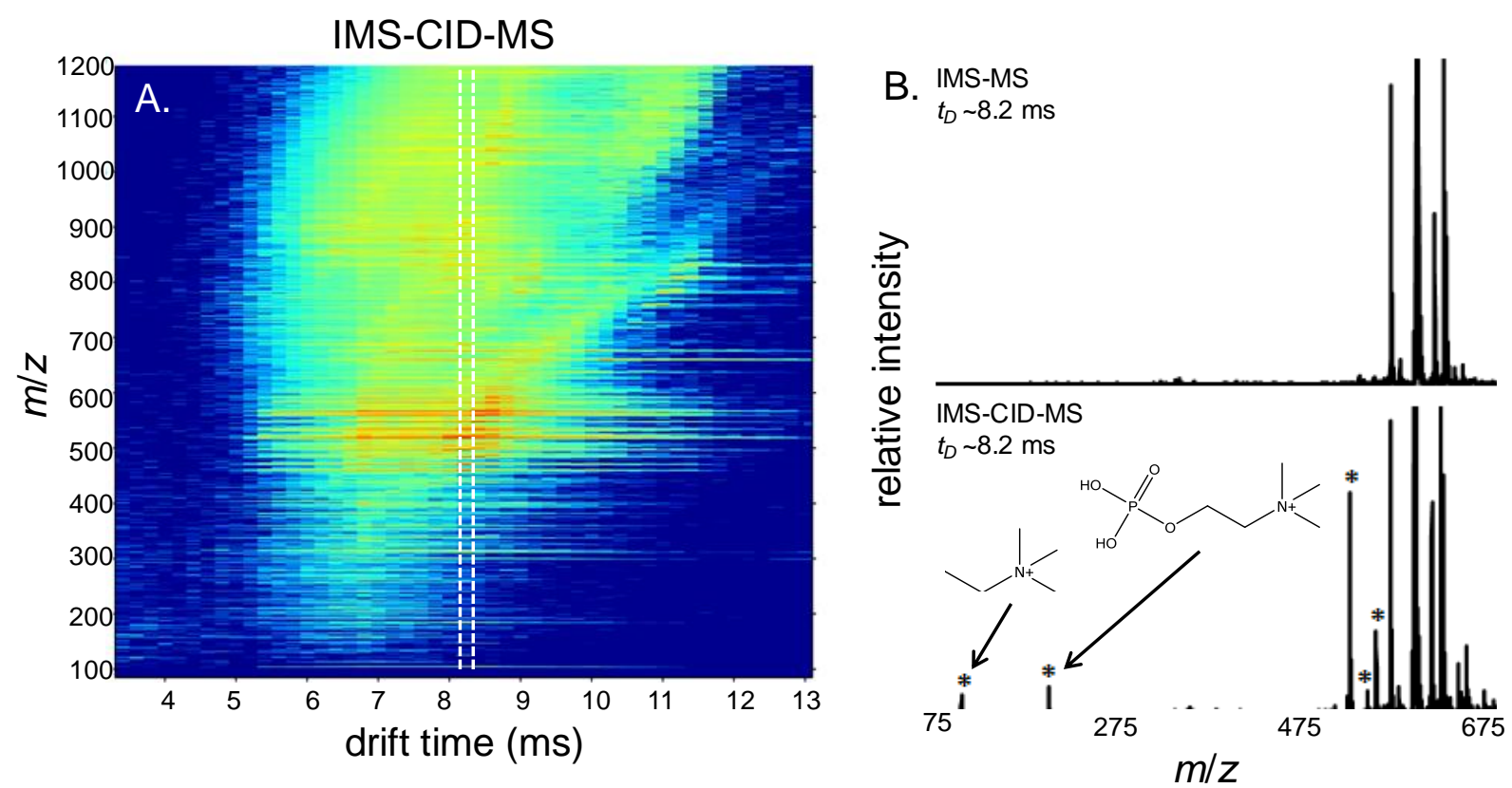

Figure 2.9 (A) Two-dimensional (2D) $t_{D}(\mathrm{~m} / \mathrm{z})$ heat-plot of the EXP24 sample obtained from an IMS-CID-MS experiment. The color map for the plot is shown on the same scale as described in Figure 1. (B) Comparison of the mass spectra produced after IMS-MS (top) and IMS-CID-MS (bottom). The white dashed line in panel A denotes the mass spectra extracted in the bottom panel B. the starred peaks indicate new spectral features not observed in IMS-MS experiments of thsame sample. Several new spectral features have been identified.

dataset for the EXP24 metabolite extract sample. Here, increased ion intensity is observed in high- and low-m/z regions indicating ion dissociation. A comparison of the mass spectra extracted at $t_{D^{\sim}} 8.2 \mathrm{~ms}$ is shown in Figure 2.9B and $\mathrm{C}$. The top panel in Figure 2.9B shows that the extracted mass spectrum $\left(t_{D} \sim 8.2 \mathrm{~ms}\right)$ is mostly void of detectable spectral features below $\mathrm{m} / z \sim 350$. Because of the mobility separation, increase analytical space is afforded and is advantageous during IMS-CID experiments. For example, IMS-CID-MS of the EXP24 sample resulted in new spectral features observed in the lower mass spectrum (Figure 2.9C), which are free of spectral interferences at $t_{D} \sim 8.2 \mathrm{~ms}$. It therefore becomes instructive to identify the fragment ions and their origin. 
A particular advantage of IMS is the ability to separate ions based on molecular class. McLean and coworks showed that lipid classes share similar functionality and thus intrinsic gas phase conformations.[72] With this in mind, Figure $2.9 \mathrm{C}$ shows that several spectral features are consistent with LysoPC molecules at $t_{D} \sim 8.2 \mathrm{~ms}$. Here, $\mathrm{m} / \mathrm{z}$ values consistent with dissociation of the choline head region and phosphodiester bond are observed in Figure 2.9. Notably, these ions also observed during $\mathrm{MS}^{\mathrm{n}}$ experiments (see above) on mobility selected ( $t_{D} \sim 8.2 \mathrm{~ms}$ ) and tentatively identified LysoPC molecules (Table 2.1). Taken collective, these results suggest that the IMS-CID-MS approach can be utilized to aid in identifying molecular classes not only on intrinsic structural packing, but also through fragmentation characteristics using IMS-CID.
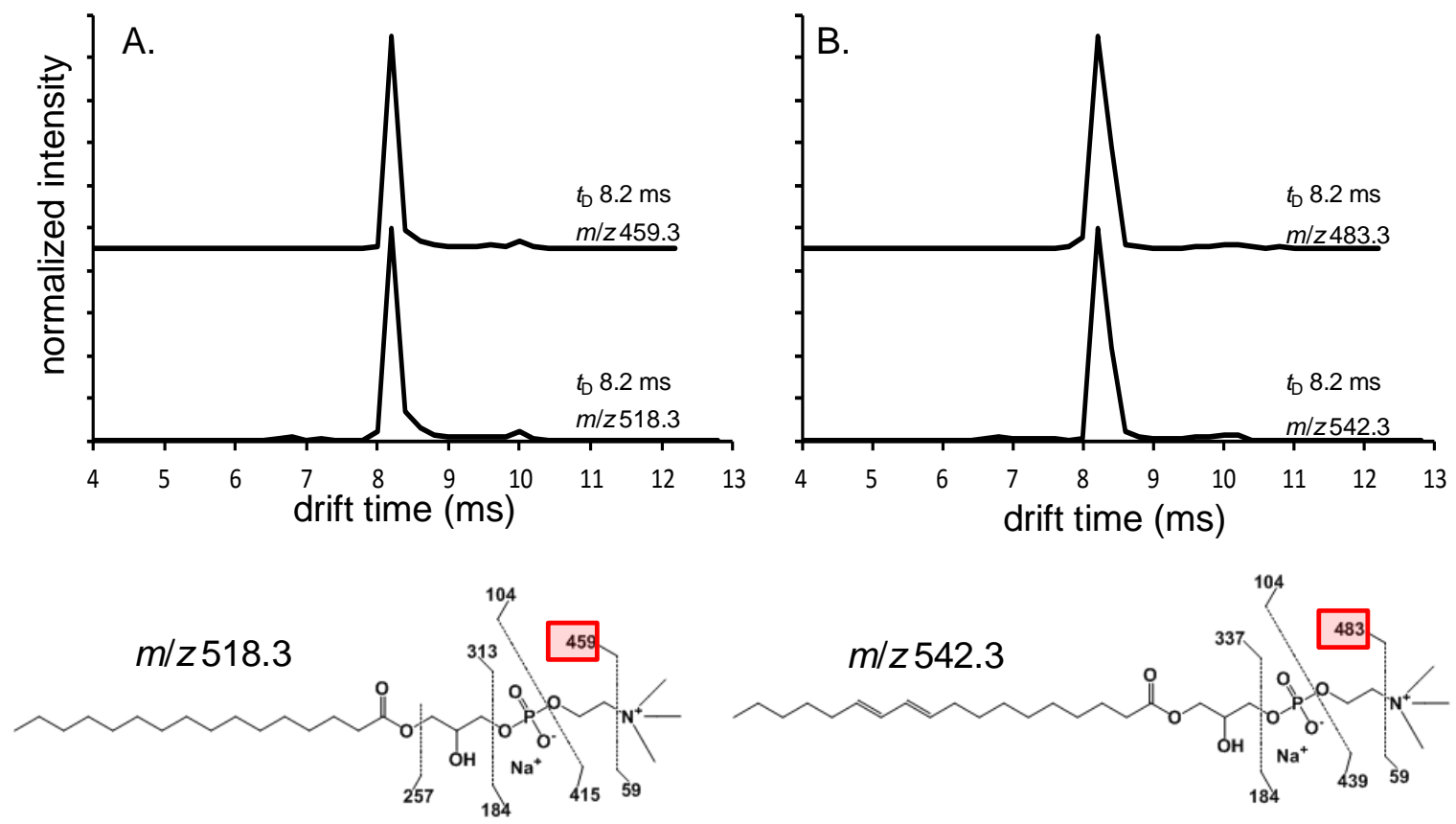

Figure 2.10 (A) XIDTD for the fragment ion $\mathrm{m} / \mathrm{z} 459.3$ (top) and precursor ion $\mathrm{m} / \mathrm{z} 518.3$ (bottom). (B) XIDTD for the fragment ion $\mathrm{m} / \mathrm{z} 483.3$ and precursor ion $\mathrm{m} / \mathrm{z} 542.3$ for top and bottom panels respectively. All XIDTDs were collected during from IMS-CID-MS of a single EXP24replicate

For complex mixtures, the data in Figure 2.9A show the challenge in associating fragment and precursor ions by IMS-CID due to increased spectral complexity. While 
some ions can be identified as unique to a particular lipid class, assigning the origin of fragment ions is more difficult. One method that may help alleviate this problem is comparison of XIDTDs. The utility of this approach for distinguishing precursor ions with overlapping mobilities has been shown previously in IMS-photodissociation (PD)-MS experiments.[42] Figure 2.10A shows an example comparison of the XIDTD for the precursor ion of $\mathrm{m} / \mathrm{z} 518.3$ (bottom panel) and the XIDTD for the fragment ion of $\mathrm{m} / \mathrm{z}$ 459.3 (top panel). Overall the two distributions are very similar. Each exhibits a major peak maximum at a $t_{D}$ value of $\sim 8.2 \mathrm{~ms}$. In each XIDTD a second peak a $t_{D}$ of $\sim 10.0 \mathrm{~ms}$ is also observed. Differences are observed in that the XIDTD of the precursor ion exhibits a small feature at a $t_{D} \sim 6.8 \mathrm{~ms}$. Another example of XIDTD matching is shown in Figure 2.10 B. Here, the XIDTD for the fragment ion $\mathrm{m} / \mathrm{z} 483.3$ is matched with the precursor XIDTD for $\mathrm{m} / \mathrm{z}$ 542.2. Overall, the XIDTDs for $\mathrm{m} / \mathrm{z} 483.3$ and $\mathrm{m} / \mathrm{z} 542.3$ are also very similar. For example, the maximum peak intensity is observed at $t_{D} \sim 8.2 \mathrm{~ms}$, followed by a smaller spectral feature at $t_{D} \sim 10.0 \mathrm{~ms}$. Also noted is the slightly broader distribution observed for these XIDTDs (Figure 2.10B).

It is instructive to compare panels $A$ and $B$ in Figure 2.10 since both precursor ions display similar spectral features $\left(t_{D}\right.$ values $\sim 8.2 \mathrm{~ms}$ and $\left.\sim 10.0 \mathrm{~ms}\right)$. Here, both fragment ions are show similar features; however, the overall XIDTDs for each fragment ion appears to correctly reflect the drift profile of the corresponding precursor ion. This is largely attributed to the dual gate design, where mobility information is passed from the precursor ion to the fragment ion before mass analysis. Using the IMS-CID-MS in a high throughput fashion, the method appears specific. That is, XIDTDs between precursor and fragment ion are capable of being match base on coincidence in drift time. It is also 
noted that no spectral alignment was made between these datasets (Figure 2.10A and B). Another observation is that for each panel in Figure $2.10 \mathrm{~A}$ and $\mathrm{B}$, the same fragment ions were generated during MS/MS (Figure 2.8A and 2.8B) of the respective precursors ions. This adds a layer of confidence in the XIDTD assignments.
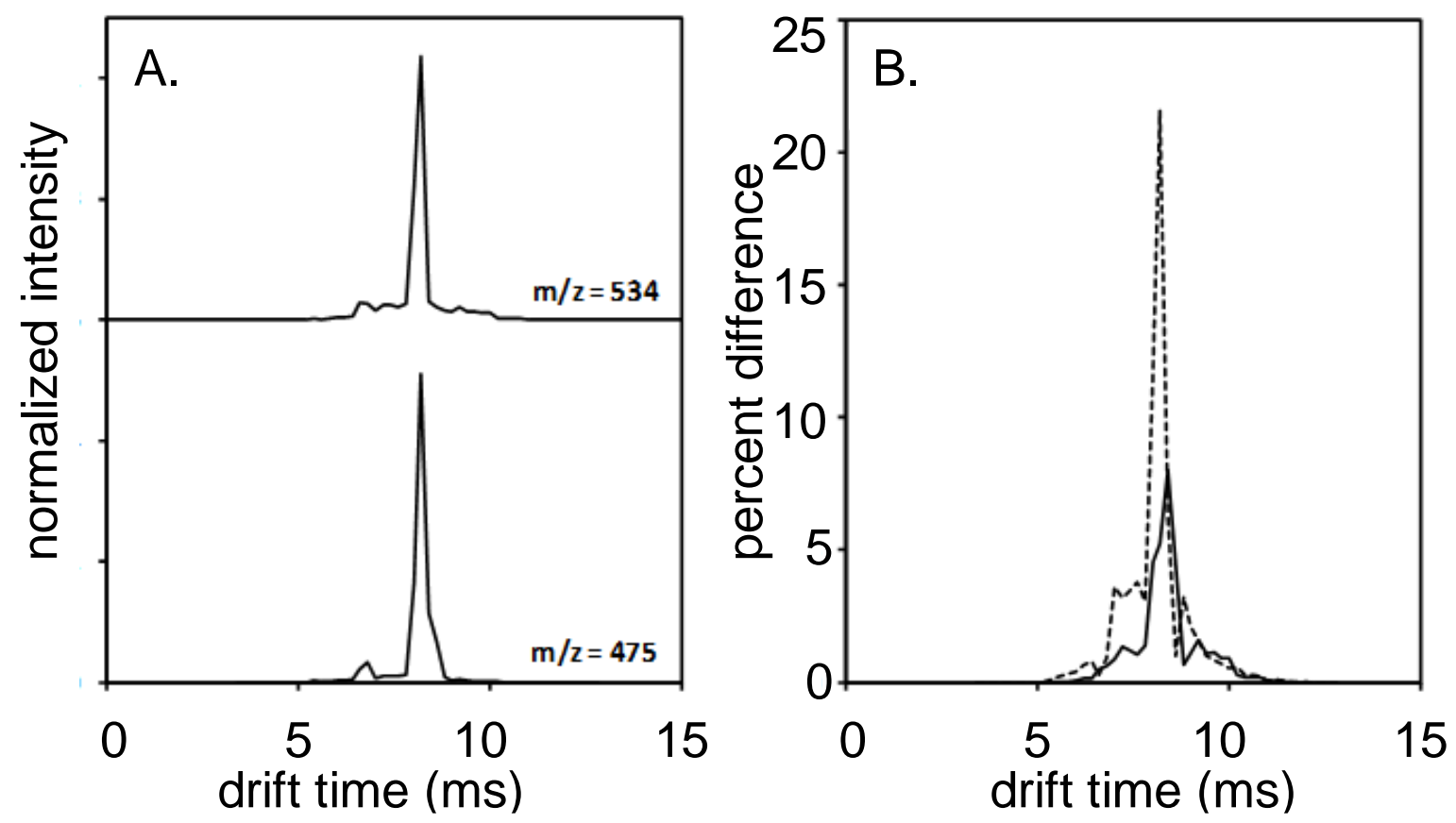

Figure 2.11 (A) XIDTD (top trace) and XIDTD (bottom trace) for the precursor and fragment ions of $\mathrm{m} / \mathrm{z} 534.2$ and 475.0, respectively. (B) Percent difference plots obtained upon comparison of the XIDTD for the fragment ion of $\mathrm{m} / \mathrm{z} 475.0$ and the XIDTDs of the precursor ions of $\mathrm{m} / \mathrm{z} 534.2$ (solid trace) and 496.3 (dashed-line trace). Each distribution is normalized such that the total area under the curve is unity.

A semi-quantitative assessment can be performed to rank the comparison of two XIDTDs. As a proof-of-principle study, Figure 2.11A shows the fragment ion of $\mathrm{m} / \mathrm{z}$ 475.2 that has been matched to $\mathrm{m} / z 534.2$ precursor ions in a similar manner as described above. Figure 2.11B shows the percent difference in XIDTDs for two different precursor ions $(\mathrm{m} / z 496.3$ and 534.2) relative to the XIDTD of the fragment ion $(\mathrm{m} / \mathrm{z}$ 475.2). The percent difference in Figure 2.11B for $m / z 534.2$ (solid trace) has a smaller integrated intensity ( $\sim 3$ times) relative to $\mathrm{m} / z 496.3$ (dashed trace). Accurate mass matching in Table 2.1 show the precursor ion of $\mathrm{m} / z 534.2$ is consistent with potassiated 
LysoPC(16:0). The IMS-CID-MS product ion of $\mathrm{m} / z 475.2$ suggests the loss of the methylated ammonium in the choline head group for this tentative assignment.

\subsubsection{Instrumental Implication for Comparative Analsyses}

Although the data provided here present a proof-of-principle demonstration of instrumentation capabilities, several improvements are envisioned to yield more effective comparative analyses. Increased IMS resolving power would better disperse the overlapping ( $t_{D}$ dimension) precursor ions (Figure 2.10) and allow for higher accuracy association of fragment ions to precursor ions in parallel dissociation experiments. Additionally, adaptations in drift tube design would allow for fragmentation of mobility-selected precursor ions followed by parallel dissociation and mobility resolution of fragment ions as demonstrated previously.[30, 38] An additional enhancement can be obtained by coupling off-line LC fractionation with the IMS-MS analysis. Because the IMS separation occurs after the ionization step, ion suppression contributes to the observation of the very high-signal LysoPC species observed in the middle range of the mass spectrum (Figure 2.5) along with the observation of much lower intensity species in other spectral regions. 


\subsection{References}

1. Valentine, S.J., et al., Gas-phase separations of protease digests. Journal of the American Society for Mass Spectrometry, 1998. 9(11): p. 1213-1216.

2. Gillig, K.J., et al., Coupling high-pressure MALDI with ion mobility/orthogonal time-of flight mass spectrometry. Analytical Chemistry, 2000. 72(17): p. 3965-3971.

3. Ruotolo, B.T., et al., Peak capacity of ion mobility mass spectrometry: Separation of peptides in helium buffer gas. Journal of Chromatography B-Analytical Technologies in the Biomedical and Life Sciences, 2002. 782(1-2): p. 385-392.

4. $\mathrm{Wu}, \mathrm{C}$., et al., Separation of isomeric peptides using electrospray ionization/high-resolution ion mobility spectrometry. Anal Chem, 2000. 72(2): p. 391-5.

5. Clowers, B.H., et al., Separation of sodiated isobaric disaccharides and trisaccharides using electrospray ionization-atmospheric pressure ion mobility-time of flight mass spectrometry. Journal of the American Society for Mass Spectrometry, 2005. 16(5): p. 660-669.

6. McLean, J.A., et al., Ion mobility-mass spectrometry: a new paradigm for proteomics. International Journal of Mass Spectrometry, 2005. 240(3): p. 301-315.

7. Valentine, S.J., et al., Toward Plasma Proteome Profiling with Ion Mobility-Mass Spectrometry. Journal of Proteome Research, 2006. 5(11): p. 2977-2984.

8. Becker, C., F.A. Fernandez-Lima, and D.H. Russell, Ion Mobility-Mass Spectrometry: A Tool for Characterizing the Petroleome. Spectroscopy, 2009. 24(4): p. 38-42.

9. Dwivedi, P., et al., Metabolic profiling by ion mobility mass spectrometry (IMMS). Metabolomics, 2008. 4(1): p. 63-80.

10. Isailovic, D., et al., Profiling of human serum glycans associated with liver cancer and cirrhosis by IMS-MS. Journal of Proteome Research, 2008. 7(3): p. 1109-1117.

11. McLean, J.A., The Mass-Mobility Correlation Redux: The Conformational Landscape of Anhydrous Biomolecules. Journal of the American Society for Mass Spectrometry, 2009. 20(10): p. 17751781.

12. Kliman, M., J.C. May, and J.A. McLean, Lipid analysis and lipidomics by structurally selective ion mobility-mass spectrometry. Biochimica Et Biophysica Acta-Molecular and Cell Biology of Lipids, 2011. 1811(11): p. 935-945.

13. Baker, E.S., et al., Ion mobility spectrometry-mass spectrometry performance using electrodynamic ion funnels and elevated drift gas pressures. J Am Soc Mass Spectrom, 2007. 18(7): p. 1176-87.

14. Dwivedi, P., A.J. Schultz, and H.H. Hill, Metabolic profiling of human blood by high-resolution ion mobility mass spectrometry (IM-MS). International Journal of Mass Spectrometry, 2010. 298(13): p. 78-90.

15. Zinnel, N.F., P.J. Pai, and D.H. Russell, Ion Mobility-Mass Spectrometry (IM-MS) for Top-Down Proteomics: Increased Dynamic Range Affords Increased Sequence Coverage. Analytical Chemistry, 2012. 84(7): p. 3390-3397.

16. Counterman, A.E., et al., Formation of peptide aggregates during ESI: Size, charge, composition, and contributions to noise. Journal of the American Society for Mass Spectrometry, 2001. 12(9): p. 1020-1035.

17. Kurulugama, R.T.I., K; Taylor, L. The Agilent Ion Mobility Q-TOF Mass

Spectrometer System. 2014 [cited 2014 May 23,]; Available from:

http://www.chem.agilent.com/Library/technicaloverviews/Public/5991-3244EN.pdf.

18. Hernandez, D.R., et al., Ion dynamics in a trapped ion mobility spectrometer. Analyst, 2014. 139(8): p. 1913-1921. 
19. Waters. SYNAPT G2-Si High Definition Mass Spectrometry. 2014 [cited 2014 7/1]; Available from: http://www.waters.com/waters/en US/SYNAPT-G2-Si-High-Definition-MassSpectrometry/nav.htm?cid=134740622\&locale=en US.

20. Steiner, W.E., et al., Atmospheric pressure matrix-assisted laser desorption/ionization with analysis by ion mobility time-of-flight mass spectrometry. Rapid Communications in Mass Spectrometry, 2004. 18(8): p. 882-888.

21. Hoaglund, C.S., et al., Three-dimensional ion mobility TOFMS analysis of electrosprayed biomolecules. Analytical Chemistry, 1998. 70(11): p. 2236-2242.

22. Scheubert, K., et al., Computing fragmentation trees from metabolite multiple mass spectrometry data. J Comput Biol, 2011. 18(11): p. 1383-97.

23. Cao, M., K. Fraser, and S. Rasmussen, Computational Analyses of Spectral Trees from Electrospray Multi-Stage Mass Spectrometry to Aid Metabolite Identification. Metabolites, 2013. 3(4): p. 1036-1050.

24. Prien, J.M., et al., The high mannose glycans from bovine ribonuclease $B$ isomer characterization by ion trap MS. J Am Soc Mass Spectrom, 2009. 20(4): p. 539-56.

25. Jiao, J., H. Zhang, and V.N. Reinhold, High Performance IT-MS Sequencing of Glycans (Spatial Resolution of Ovalbumin Isomers). Int J Mass Spectrom, 2011. 303(2-3): p. 109-117.

26. Bluhm, B.K., K.J. Gillig, and D.H. Russell, Development of a Fourier-transform ion cyclotron resonance mass spectrometer-ion mobility spectrometer. Review of Scientific Instruments, 2000. 71(11): p. 4078-4086.

27. Clowers, B.H. and H.H. Hill, Mass analysis of mobility-selected ion populations using dual gate, ion mobility, quadrupole ion trap mass spectrometry. Analytical Chemistry, 2005. 77(18): p. 5877-5885.

28. Tang, X., J.E. Bruce, and H.H. Hill, Jr., Design and performance of an atmospheric pressure ion mobility Fourier transform ion cyclotron resonance mass spectrometer. Rapid Commun Mass Spectrom, 2007. 21(7): p. 1115-22.

29. Zucker, S.M., et al., An Ion Mobility/Ion Trap/Photodissociation Instrument for Characterization of Ion Structure. Journal of the American Society for Mass Spectrometry, 2011. 22(9): p. 14771485.

30. Li, H.L., et al., Carbohydrate Structure Characterization by Tandem Ion Mobility Mass Spectrometry (IMMS)(2). Analytical Chemistry, 2013. 85(5): p. 2760-2769.

31. Knorr, F.J., et al., FOURIER-TRANSFORM ION MOBILITY SPECTROMETRY. Analytical Chemistry, 1985. 57(2): p. 402-406.

32. Clowers, B.H., et al., Hadamard transform ion mobility spectrometry. Anal Chem, 2006. 78(1): p. 44-51.

33. Szumlas, A.W., S.J. Ray, and G.M. Hieftje, Hadamard transform ion mobility spectrometry. Anal Chem, 2006. 78(13): p. 4474-81.

34. Tang, K., et al., High-sensitivity ion mobility spectrometry/mass spectrometry using electrodynamic ion funnel interfaces. Analytical Chemistry, 2005. 77(10): p. 3330-3339.

35. Koeniger, S.L., et al., An IMS-IMS analogue of MS-MS. Analytical Chemistry, 2006. 78(12): p. 4161-4174.

36. Valentine, S.J., S.L. Koeniger, and D.E. Clemmer, A split-field drift tube for separation and efficient fragmentation of biomolecular ions. Analytical Chemistry, 2003. 75(22): p. 6202-6208.

37. Koeniger, S.L., et al., Development of field modulation in a split-field drift tube for highthroughput multidimensional separations. Journal of Proteome Research, 2005. 4(1): p. 25-35.

38. Merenbloom, S.I., et al., IMS-IMS and IMS-IMS-IMS/MS for separating peptide and protein fragment ions. Analytical Chemistry, 2006. 78(8): p. 2802-2809. 
39. Syka, J.E.P., et al., Peptide and protein sequence analysis by electron transfer dissociation mass spectrometry. Proceedings of the National Academy of Sciences of the United States of America, 2004. 101(26): p. 9528-9533.

40. Beausoleil, S.A., et al., Large-scale characterization of HeLa cell nuclear phosphoproteins. Proc Natl Acad Sci U S A, 2004. 101(33): p. 12130-5.

41. Schroeder, M.J., et al., A neutral loss activation method for improved phosphopeptide sequence analysis by quadrupole ion trap mass spectrometry. Anal Chem, 2004. 76(13): p. 3590-8.

42. Lee, S., et al., Extracted fragment ion mobility distributions: A new method for complex mixture analysis. International Journal of Mass Spectrometry, 2012. 309: p. 154-160.

43. Hoadlund-Hyzer, C.S., J.W. Li, and D.E. Clemmer, Mobility labeling for parallel CID of ion mixtures. Analytical Chemistry, 2000. 72(13): p. 2737-2740.

44. Stone, E., et al., Surface-induced dissociation on a MALDI-ion mobility-orthogonal time-of-flight mass spectrometer: Sequencing peptides from an "in-solution" protein digest. Analytical Chemistry, 2001. 73(10): p. 2233-2238.

45. Nurkiewicz, T., et al., Nanoparticle inhalation augments particle-dependent systemic microvascular dysfunction. Particle and Fibre Toxicology, 2008. 5(1): p. 1.

46. Knuckles, T.L., et al., Nanoparticle inhalation alters systemic arteriolar vasoreactivity through sympathetic and cyclooxygenase-mediated pathways. Nanotoxicology, 2012. 6(7): p. 724-735.

47. Fenn, J.B., et al., ELECTROSPRAY IONIZATION FOR MASS-SPECTROMETRY OF LARGE BIOMOLECULES. Science, 1989. 246(4926): p. 64-71.

48. Revercomb, H.E. and E.A. Mason, THEORY OF PLASMA CHROMATOGRAPHY GASEOUS ELECTROPHORESIS - REVIEW. Analytical Chemistry, 1975. 47(7): p. 970-983.

49. Shvartsburg, A.A. and M.F. Jarrold, An exact hard-spheres scattering model for the mobilities of polyatomic ions. Chemical Physics Letters, 1996. 261(1-2): p. 86-91.

50. Mesleh, M.F., et al., Structural Information from lon Mobility Measurements: Effects of the Long-Range Potential. The Journal of Physical Chemistry, 1996. 100(40): p. 16082-16086.

51. Wyttenbach, T., et al., Effect of the long-range potential on ion mobility measurements. Journal of the American Society for Mass Spectrometry, 1997. 8(3): p. 275-282.

52. Wittmer, D., et al., ELECTROSPRAY-IONIZATION ION MOBILITY SPECTROMETRY. Analytical Chemistry, 1994. 66(14): p. 2348-2355.

53. Clemmer, D.E., R.R. Hudgins, and M.F. Jarrold, NAKED PROTEIN CONFORMATIONS CYTOCHROME-C IN THE GAS-PHASE. Journal of the American Chemical Society, 1995. 117(40): p. 10141-10142.

54. von Helden, G., T. Wyttenbach, and M.T. Bowers, Conformation of macromolecules in the gas phase: use of matrix-assisted laser desorption methods in ion chromatography. Science, 1995. 267(5203): p. 1483-5.

55. Hoaglund-Hyzer, C.S. and D.E. Clemmer, Ion trap/ion mobility/quadrupole/time-of-flight mass spectrometry for peptide mixture analysis. Anal Chem, 2001. 73(2): p. 177-84.

56. Merenbloom, S.I., et al., High-resolution ion cyclotron mobility spectrometry. Anal Chem, 2009. 81(4): p. 1482-7.

57. Kurulugama, R.T., et al., Overtone Mobility Spectrometry: Part 1. Experimental Observations. Journal of the American Society for Mass Spectrometry, 2009. 20(5): p. 729-737.

58. Blase, R.C., et al., Increased ion transmission in IMS: A high resolution, periodic-focusing DC ion guide ion mobility spectrometer. International Journal of Mass Spectrometry, 2011. 301(1-3): p. 166-173.

59. Stlouis, R.H. and H.H. Hill, ION MOBILITY SPECTROMETRY IN ANALYTICAL-CHEMISTRY. Critical Reviews in Analytical Chemistry, 1990. 21(5): p. 321-355. 
60. Clemmer, D.E. and M.F. Jarrold, Ion mobility measurements and their applications to clusters and biomolecules. Journal of Mass Spectrometry, 1997. 32(6): p. 577-592.

61. Collins, D.C. and M.L. Lee, Developments in ion mobility spectrometry-mass spectrometry. Analytical and Bioanalytical Chemistry, 2002. 372(1): p. 66-73.

62. Bohrer, B.C., et al., Biomolecule Analysis by Ion Mobility Spectrometry, in Annual Review of Analytical Chemistry. 2008. p. 293-327.

63. Shaffer, S.A., et al., A novel ion funnel for focusing ions at elevated pressure using electrospray ionization mass spectrometry. Rapid Communications in Mass Spectrometry, 1997. 11(16): p. 1813-1817.

64. Ruotolo, B.T., et al., Distinguishing between phosphorylated and nonphosphorylated peptides with ion mobility-mass spectrometry. J Proteome Res, 2002. 1(4): p. 303-6.

65. Ruotolo, B.T., et al., Analysis of phosphorylated peptides by ion mobility-mass spectrometry. Anal Chem, 2004. 76(22): p. 6727-33.

66. Palumbo, A.M., J.J. Tepe, and G.E. Reid, Mechanistic insights into the multistage gas-phase fragmentation behavior of phosphoserine- and phosphothreonine-containing peptides. J Proteome Res, 2008. 7(2): p. 771-9.

67. Palumbo, A.M., et al., Tandem mass spectrometry strategies for phosphoproteome analysis. Mass Spectrom Rev, 2011. 30(4): p. 600-25.

68. Wishart, D.S., et al., HMDB 3.0-The Human Metabolome Database in 2013. Nucleic Acids Research, 2013. 41(D1): p. D801-D807.

69. Fang, N., S. Yu, and T.M. Badger, LC-MS/MS Analysis of Lysophospholipids Associated with Soy Protein Isolate. Journal of Agricultural and Food Chemistry, 2003. 51(23): p. 6676-6682.

70. Barroso, B. and R. Bischoff, LC-MS analysis of phospholipids and lysophospholipids in human bronchoalveolar lavage fluid. Journal of Chromatography B, 2005. 814(1): p. 21-28.

71. Liu, X.Y., et al., Mapping the human plasma proteome by SCX-LC-IMS-MS. Journal of the American Society for Mass Spectrometry, 2007. 18(7): p. 1249-1264.

72. May, J.C., et al., Conformational Ordering of Biomolecules in the Gas Phase: Nitrogen Collision Cross Sections Measured on a Prototype High Resolution Drift Tube Ion Mobility-Mass Spectrometer. Analytical Chemistry, 2014. 86(4): p. 2107-2116. 


\section{Negative Ion IMS-HDX-MS ${ }^{1}$}

${ }^{1}$ Reprinted with permission from the Journal of the American Society for Mass Spectrometry: Ion Mobility Spectrometry-Hydrogen Deuterium Exchange Mass Spectrometry of Anions: Part 1 Peptides to Proteins. Gregory C. Donohoe, Mahdiar Khakinejad, Stephen J. Valentine, J. Am. Soc. Mass Spectrom., 2015, 26 (4), 564-576.

\subsection{Introduction: A Base for Studying Anionic Proteins}

The development of soft ionization methods such as electrospary ionization (ESI)[1] and matrix-assisted laser desorption (MALDI)[2, 3] presented the unique opportunity to study the gas-phase conformations of large biomolecular ions. Within a short time a variety of mass spectrometry (MS)-based techniques were developed for the structural characterization of these ions as well as to relate gas-phase conformers to antecedent solution structures.[4-20] One early technique determined the gas-phase hydrogen-deuterium exchange (HDX) reactivities of ions comprising different charge states of proteins.[6, 21-24] The observation of different HDX rates and maximum HDX levels indicated the presence of coexisting gas-phase conformations. Early theoretical studies resulted in proposed mechanisms for HDX of protonated peptide ions by a number of deuterating agents. [8, 22]

Concurrent to the development of HDX techniques, experimental efforts were directed at utilizing ion mobility spectrometry (IMS) coupled with MS for the determination of collision cross sections for peptide and protein ions.[9, 19, 20, 25] Shortly later, IMS-MS was combined with HDX in order to determine the reactivities of specific gas-phase conformers of protein ions. These early IMS-HDX-MS experiments provided information about the degree of protection afforded by the protein fold 
associated with elongated and compact conformations for positively charged biomolecules.

Relative to positive ions, anionic peptides and proteins have received considerably less attention.[26] As a consequence, very few studies have been performed on negation ions. Because many proteins contain a large number of acidic residues, it is useful to develop a sound understanding of the conformational information afforded by gas-phase HDX measurements for select conformers from negativelycharged protein ions. Similarly the combined IMS-HDX-MS studies described here present the first conformer specific analysis of peptide and protein anions. That said, the methods utilized in these studies are similar to those employed previously for the characterization of conformers of positively-charged protein ions.[27, 28]

\subsection{Method and Materials}

\subsubsection{Sample Preparation}

Samples were selected to allow comparisons of a variety of gas-phase ion conformers including those formed from a conformationally-restricted protein (disulfide bonds) and globular proteins of increasing size. The proteins bovine insulin (90\%), ubiquitin (95\%), and equine cytochrome c (95\%) were purchased (Sigma Aldrich, St. Louis, MO, USA) and used without further purification. Ultra-pure (chromatography grade) deionized water, acetonitrile, and ammonium hydroxide (Fisher Scientific, Fair Lawn, NJ, USA) were used to generate stock and ESI solutions of the peptide and proteins. The stock solutions were prepared by dissolving $1.0 \mathrm{mg}$ of analyte in $1.0 \mathrm{~mL}$ of ultra-pure water and were capped in glass vials while being maintained in a refrigerator $\left(4{ }^{\circ} \mathrm{C}\right)$; no stock solution was used a week after preparation. ESI solutions $(0.1 \mathrm{mg} \cdot \mathrm{mL}$ 
${ }^{1}$ ) were prepared fresh by diluting $0.1 \mathrm{~mL}$ of stock solution with the addition of $0.4 \mathrm{~mL}$ of ammonium hydroxide and $0.5 \mathrm{~mL}$ of acetonitrile. Samples were infused through a pulled-tip capillary using a flow rate of $300 \mathrm{~nL} \cdot \mathrm{min}^{-1}$.

\subsubsection{IMS-MS: Instrumental Operation}

The use of ion mobility spectrometry for the characterization of biomolecules has an extensive history; the development of IMS instrumentation,[19, 25, 29-45] theory[4657] and applications[58-62] have been discussed in detail. The IMS-MS instrument used in these studies has also been described previously.[63] A brief description of the instrument and data collection and analysis is presented here. Figure 1.1 shows a schematic diagram of the drift tube, linear ion trap instrument used to conduct these experiments. Peptide/Protein ions are generated by electrospraying the ESI solution through a pulled-tip capillary. The capillary is biased at $\sim-2200 \mathrm{~V}$ above the entrance aperture of the desolvation region (Figure 1.1). Here ions are desolvated and focused into an "hour-glass" ion funnel [64] (F1/IA1/G1 in Figure 1.1). lons are trapped near the exit region of the ion funnel and periodically (typically $50 \mathrm{~Hz}$ ) pulsed into the drift tube. The drift tube is filled with He buffer gas $(300 \mathrm{~K})$ and supports a drift field of $\sim 10 \mathrm{~V} \cdot \mathrm{cm}^{-1}$. lons separate in the drift tube based on differences in their mobilities through the buffer gas. The second ion gate (G2 in Figure 1.1) is employed to select ions of specific mobilities for transmission into the LTQ Velos linear ion trap (ThermoScientific, San Jose, CA, USA) for mass analysis.

3.2.2.1. Recording Two - Dimensional (2D) $t_{\underline{D}}(\mathrm{~m} / \mathrm{z})$ Distributions

Delay times between the high-voltage pulse applied to the drift tube entrance gate (G1) and the mobility selection gate (G2) are scanned in order to generate IMS-MS 
datasets. In the current instrument G1 is a single gridded lens and G2 is a Tyndall gate located directly in front of the second ion funnel. G1 prevents ions from entering the drift tube using an $\sim 40 \mathrm{~V}$ bias relative to first ion funnel exit. Periodically, a $150 \mu$ s-long voltage pulse is applied to $\mathrm{G} 1$ to allow ions to enter the drift tube. The Tyndall gate, with a bias of $\sim 10 \mathrm{~V}$, neutralizes all ions with the exception of those selected for transmission by the time delay of the voltage pulse applied to $G 2$ relative to $G 1$. The timing of G1 and G2 is synchronized by two four channel digital/delay pulse generators (Stanford Research Systems, Sunnyvale, CA, USA). Drift time $\left(t_{D}\right)$ delays are scanned from $\sim 3 \mathrm{~ms}$ to $\sim 15 \mathrm{~ms}$ (200 $\mu \mathrm{s}$ increments) to encompass the entire $t_{D}$ range of all ions produced by ESI.

\subsubsection{Mass Spectrometry Measurements}

Total ion mass spectra are generated by setting the drift tube gates to pass all ions. In this operational mode, all ions pass into the mass analyzer. The mass analyzer scan parameters include a $\mathrm{m} / \mathrm{z}$ range of 80 to 2000 as well as automatic gain control (AGC) with a threshold of $1 \times 10^{6}$ ions. For these analyses, sample injection times of 200 ms (5 microscans) have been utilized. During $t_{D}$ distribution generation, the ion gates in the drift tube are activated as mentioned above to transmit ions of a specific mobility into the linear trap. Data acquisition is accomplished by collecting a mass spectrum (0.5 min) for each $t_{D}$ selection setting. For IMS data generation, the AGC is disabled and a sample injection time of $200 \mathrm{~ms}$ (5 microscans) is also employed.

\subsubsection{Generating IMS-MS Datasets}

To generate the two-dimensional IMS-MS dataset, each $t_{D}$ selected mass spectrum (.RAW file) is converted to a separate text file. All $x$ and $y$ data points $(\mathrm{m} / \mathrm{z}$ 
values and intensities) are then associated with the respective mobility selection time $\left(t_{D}\right)$ using software developed in house. This process creates a three-column array text file containing $t_{D}, \mathrm{~m} / \mathrm{z}$, and intensity values. Intensity filters can be applied to control the size of the three-column array file. To generate $t_{D}$ distributions for ions of specific $\mathrm{m} / \mathrm{z}$ values, a separate program developed in house integrates all intensities within a userdefined $\mathrm{m} / \mathrm{z}$ range for each $t_{D}$. In accordance with previous studies, this is called an extracted ion drift time distribution (XIDTD).[65]

\subsubsection{Gas Phase HDX Experiment Design}

Gas-phase HDX is accomplished by introducing $\mathrm{D}_{2} \mathrm{O}(>99 \%$, Sigma Aldrich, St. Louis, MO, USA) into the drift tube buffer gas system. First, the $\mathrm{D}_{2} \mathrm{O}$ was purified by several freezing and melting cycles under vacuum. The pressures of $\mathrm{D}_{2} \mathrm{O}$ and helium are adjusted with two separate leak valves, (Granville Philips, Longmont, CO, USA) and monitored using a Baratron capacitance manometer (MKS, Andover, MA, USA). For these experiments, the helium pressure is set at $2.50 \pm 0.01$ Torr. The partial pressure of $\mathrm{D}_{2} \mathrm{O}$ is varied from $\sim 0.05$ Torr to $\sim 0.20$ Torr. $t_{D}$ distributions are first recorded in pure He to allow the determination of accurate collision cross sections. To ensure no conformational transitions occur, the $t_{D}$ distributions are monitored upon adding $\mathrm{D}_{2} \mathrm{O}$ as demonstrated previously [23]. Additionally, the $\mathrm{D}_{2} \mathrm{O}$ uptake for the different ions is recorded at different $\mathrm{D}_{2} \mathrm{O}$ pressures. The partial pressures of the components of the buffer gas system are stable differing by less than \pm 0.01 Torr during the course of the mass spectral characterization as well and upon removal of the $\mathrm{D}_{2} \mathrm{O}$, the total pressure quickly re-equilibrates to 2.50 Torr.

\subsubsection{HDX Levels and Rate Constants}


The number of deuteriums incorporated into specific ion conformers is determined by subtracting their average $\mathrm{m} / \mathrm{z}$ value after passing through the pure $\mathrm{He}$ buffer gas from their average $m / z$ value after passing through a $\mathrm{He} / \mathrm{D}_{2} \mathrm{O}$ mixture. Average values are determined using a simple algorithm developed in house that weights each $\mathrm{m} / \mathrm{z}$ point in the isotopic distribution by its intensity.

HDX rate constants have been determined for a number of different ion conformers assuming pseudo first order kinetics as described previously.[6, 21, 27, 28] Briefly, the partial pressure of $D_{2} \mathrm{O}$ is adjusted in small increments (as little as 0.01 Torr) from 0 to 0.20 Torr and the $t_{D}$ and HDX levels of different ion conformers are determined. A semi-log plot of the number of remaining exchangeable hydrogens as a function of the product of the $\mathrm{D}_{2} \mathrm{O}$ pressure and $t_{D}$ provides a means for determining the rate constant; the slopes of best linear fits to the data on the semi-log plot are used for this determination.

\subsection{Results and Discussion}

\subsubsection{Protein Anion Collision Cross Sections}

Figure 3.1 shows the two-dimensional $t_{D}, \mathrm{~m} / \mathrm{z}$ distribution obtained for negativelycharged ubiquitin ions. Ions produced from the ubiquitin sample range from the [M$5 \mathrm{H}]^{5-}$ to the $[\mathrm{M}-9 \mathrm{H}]^{9-}$ species. The drift times for these same ions range from $\sim 7.5$ to 13 ms. The data indicate a transition from a more elongated structural conformation type to a more compact structural type for the $[\mathrm{M}-7 \mathrm{H}]^{7-}$ and $[\mathrm{M}-6]^{6-}$ ions, respectively. This is evidenced by the higher mobility of the dominate conformer type for the latter ions 

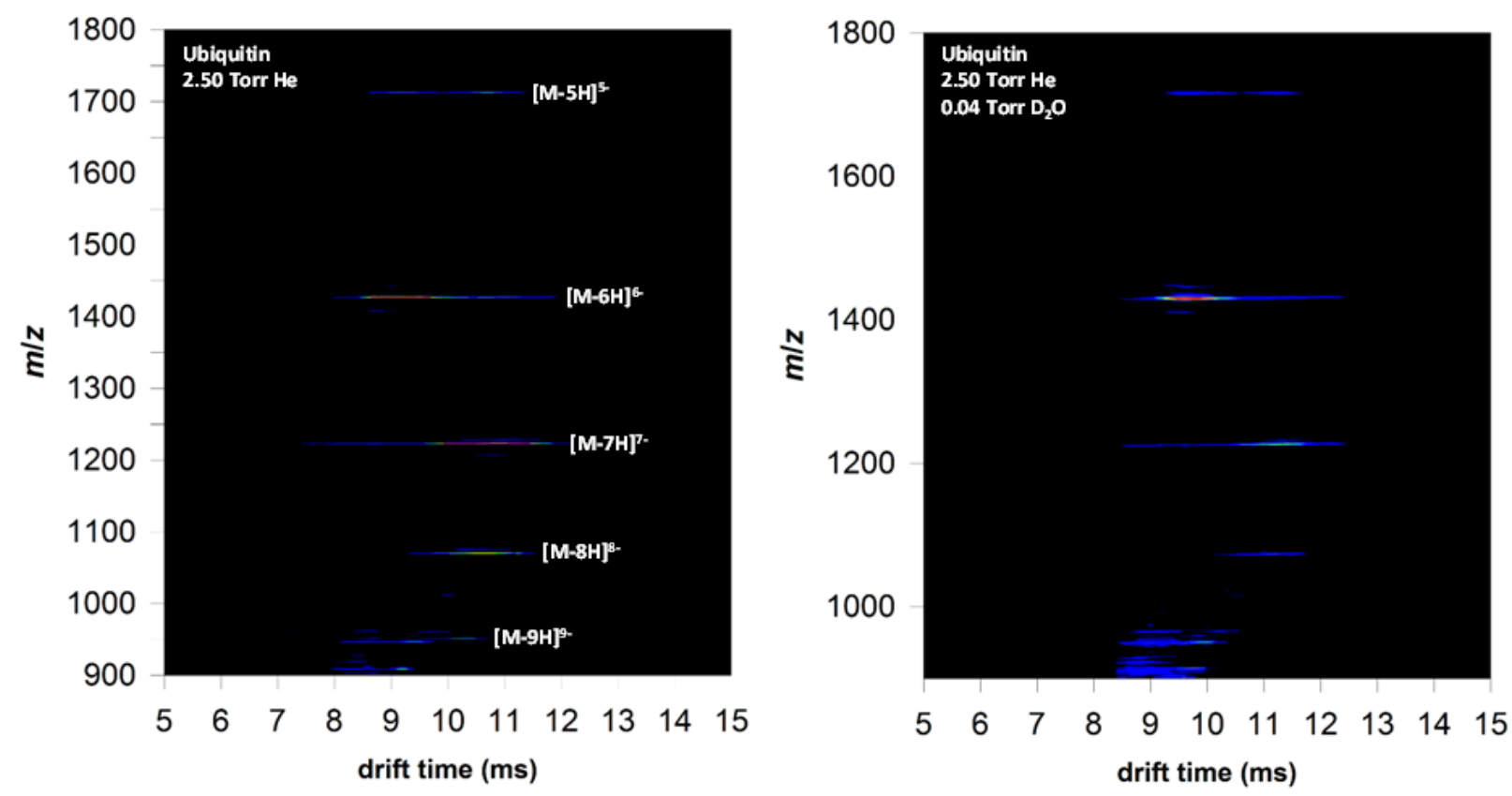

Figure 3.1 Two-dimensional, $t_{D}(\mathrm{~m} / \mathrm{z})$ dot plot of electrosprayed ubiquitin ions. The intensity is represented with a color scale using logarithmic cutoff thresholds. The maximum intensity cutoff is $10^{2}$. The ions of different charge are labeled on the two-dimensional plot.

(shorter $t_{D}$ in Figure 3.1). Ion elongation resulting from increased coulomb repulsion has been described for a number of protonated and deprotonated protein ions. [59, 6668] Figure 3.1 also shows the $t_{D}, m / z$ distribution obtained for negatively-charged ubiquitin ions upon addition of $\sim 0.04$ Torr of $\mathrm{D}_{2} \mathrm{O}$ into the drift tube buffer gas. In general the $\mathrm{m} / \mathrm{z}$ peaks become broader due to the incorporation of detueriums at heteroatom exchange sites. On average, the $t_{D}$ values of ion conformers increase by $\sim 0.5 \mathrm{~ms}$. At this $\mathrm{D}_{2} \mathrm{O}$ pressure it is expected that the more highly accessible exchange sites have incorporated deuteriums (see HDX kinetics discussion below). Although the addition of $\mathrm{D}_{2} \mathrm{O}$ does not appear to alter the conformations of the observed ions as evidenced by the similar $t_{D}$ distributions, at higher $\mathrm{D}_{2} \mathrm{O}$ pressures, the overall ion signal level decreases and maximum HDX levels and rate constants cannot be determined for some ion conformers of lower abundance (see below). 
Figure $3.2 \mathrm{~A}$ shows that collision cross section distributions indicate that several $[\mathrm{M}-\mathrm{nH}]^{\mathrm{n}-}$ ions yield two conformations (i.e., two dominant features are observed in the $t_{D}$ distribution). These collision cross sections are also summarized in Table 3.1. The collision cross sections of ions produced from bovine insulin are listed in Table 3.1 and shown in Figure 3.2B. For illustrative purposes the collision cross sections of ions from bovine insulin are depicted separately from those formed by the larger proteins (ubiquitin and cytochrome c). Insulin is observed to produce a single dimer, $[2 \mathrm{M}-7 \mathrm{H}]^{7-}$, having a cross section of $1260 \AA^{2}$. It is likely that the largest contributing factor to the relatively decreased size of the insulin ions is the presence of the four disulfide bonds which serve to tether the molecule in more compact conformations even at higher charge states. This phenomenon has been described previously.[68]

The collision cross sections for the ions formed from the larger proteins are shown in Figure 3.2A. For these ions, cross sections range in size from $862 \AA^{2}$ to 2615 $\AA^{2}$ corresponding to $[\mathrm{M}-5 \mathrm{H}]^{5-}$ ubiquitin and $[\mathrm{M}-13 \mathrm{H}]^{13-}$ cytochrome $c$ ions, respectively. Ubiquitin ions show both compact and more elongated conformations across the observed charge state distribution. A transition from the dominant species being comprised of more compact ions to more elongrated ions is observed from the $[\mathrm{M}-6 \mathrm{H}]^{6-}$ to the $[\mathrm{M}-7 \mathrm{H}]^{7-}$ species as evidenced in Figure 3.1 (see discussion above). The dataset features observed for cytochrome $c$ show only single conformer types across the charge state range (from the $[\mathrm{M}-7 \mathrm{H}]^{7-}$ to the $[\mathrm{M}-12 \mathrm{H}]^{12-}$ ions). The overlapping charge states for cytochrome $c$ and ubiquitin reflect the difference in size of the two proteins. That is, 

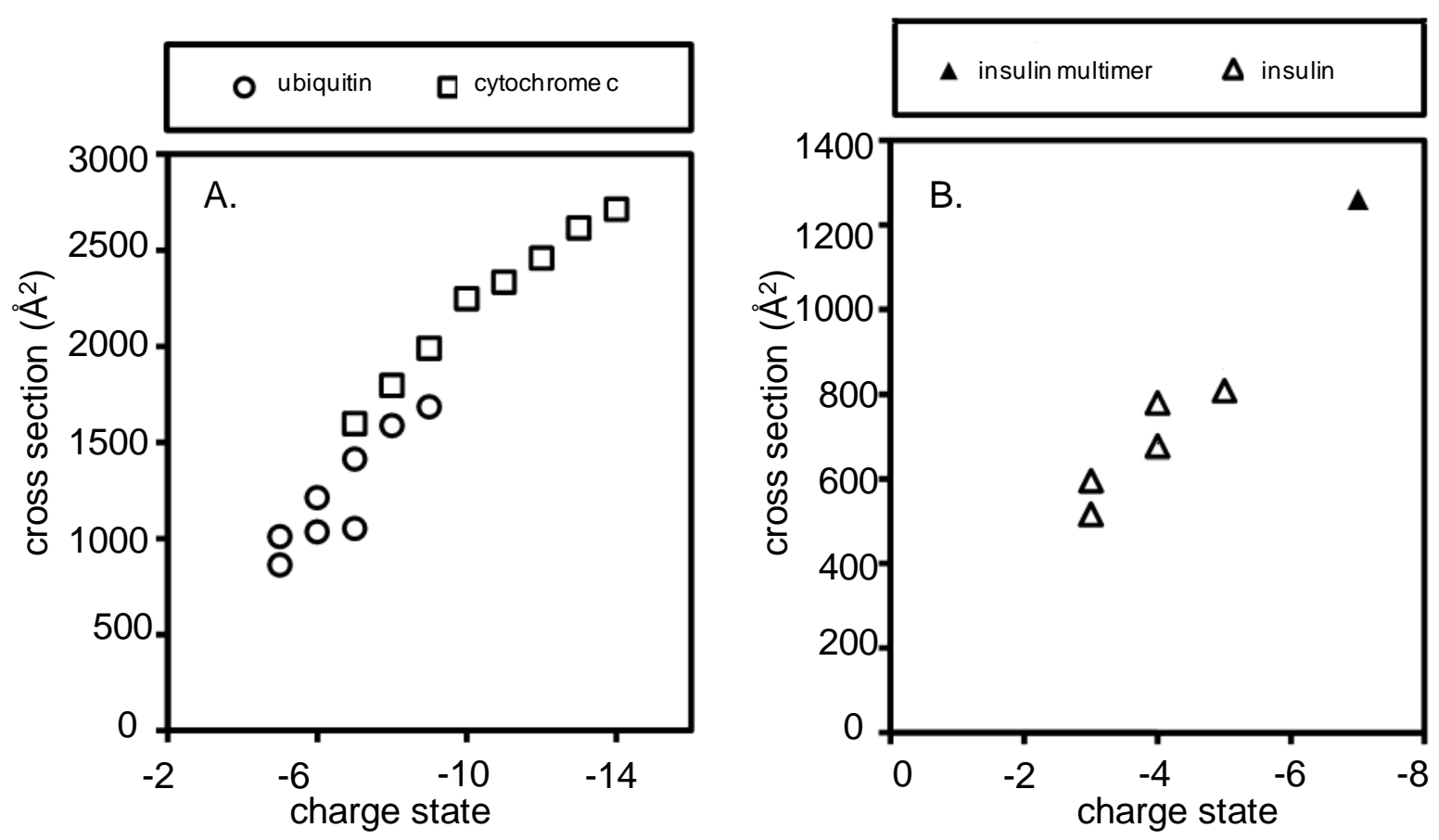

Figure 3.2. Collision cross sections for protein (panel A) and insulin (panel B) conformers. Triangle, circle and square symbols represent negatively-charged ions of the model peptide, bovine insulin, ubiquitin, and equine cytochrome c, respectively. Solid and open symbols represent monomeric and multimeric species, respectively. The number of individual monomers within multimeric species precedes the symbol.

cytochrome $c$ contains $\sim 37 \%$ more amino acid residues than ubiquitin. Additionally, no more compact ions are observed for these charge states of cytochrome $c$. The collision cross sections of the elongated cytochrome $c$ protein ions have been compared to values reported in an online database obtained from separate IMS measurements and the average percent difference is determined to be $1.7 \pm 0.3 \AA^{2}$.

\subsubsection{Gas - Phase Deuterium Uptake as a Function of $\underline{D}_{2}$ O Pressure}

The addition of $\mathrm{D}_{2} \mathrm{O}$ to the drift tube, results in shifts of the $\mathrm{m} / \mathrm{z}$ values of the various ions to higher values indicating the incorporation of deuterium. Figure $3.3 \mathrm{~A}$ shows the increase in $\mathrm{m} / \mathrm{z}$ of the more compact conformer of $[\mathrm{M}-5 \mathrm{H}]^{5-}$ ubiquitin ions as a 

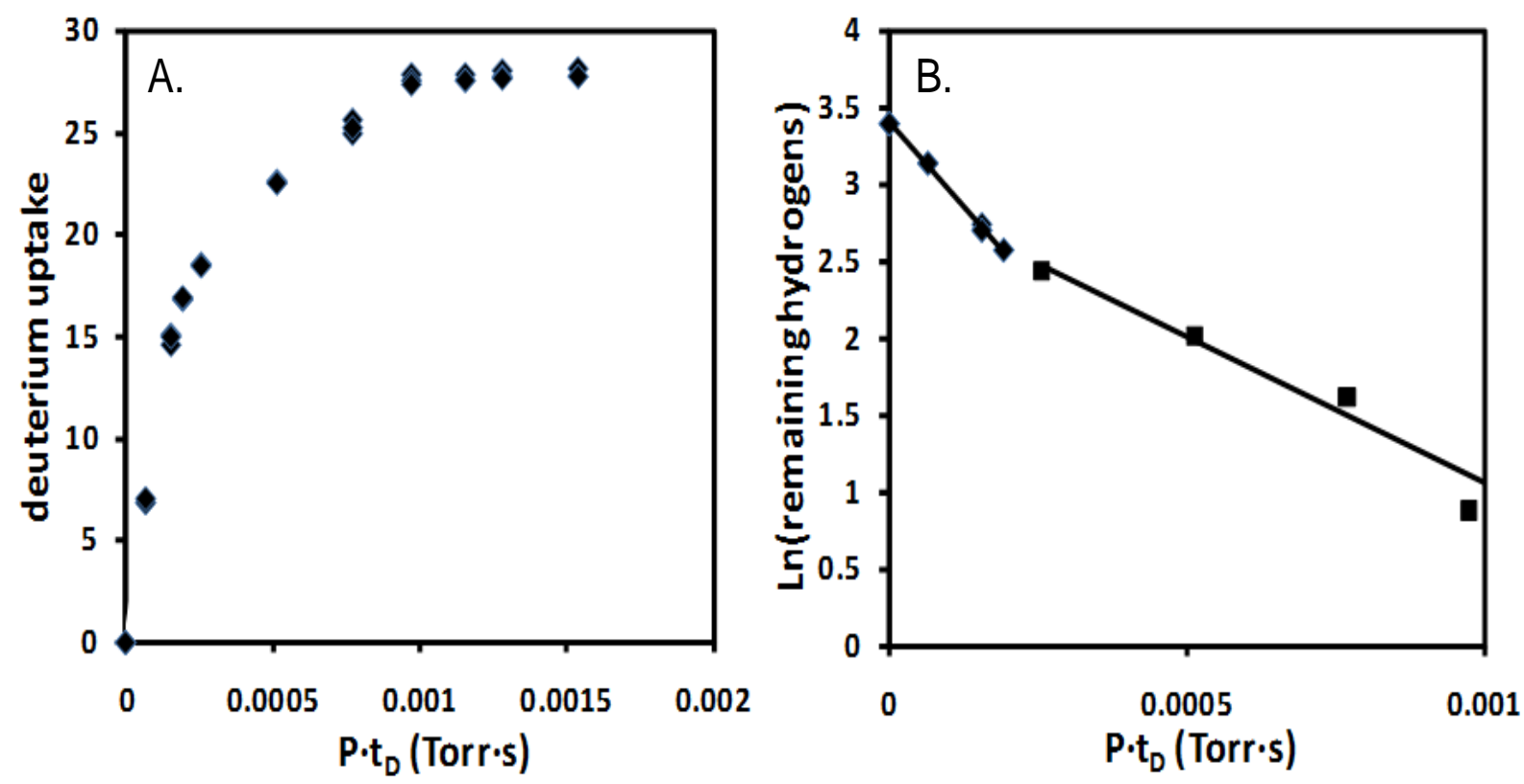

Figure 3.3. ( A) Plot of deuterium uptake as a function of the partial pressure of $D_{2} O$ multiplied by $t_{D}$. This data was recorded for $[\mathrm{M}-5 \mathrm{H}]^{5-}$ ubiquitin ions. (B) Plot of the natural log of the number of remaining exchangeable hydrogens as a function of the product of $D_{2} \mathrm{O}$ partial pressure and $t_{D}$. Linear fits of fast- and slow-exchanging hydrogens are shown.

function of the product of ion $t_{D}$ and pressure of $\mathrm{D}_{2} \mathrm{O}$. Initially, small increases in $\mathrm{D}_{2} \mathrm{O}$ pressure are associated with large increases in HDX levels while at higher $\mathrm{D}_{2} \mathrm{O}$ pressures the HDX level reaches a near maximum value corresponding to uptake of $\sim 28$ deuteriums. Using this deuterium uptake data it is possible to generate a semilog plot of the remaining hydrogens as a function of the product of pressure and ion residence time in the drift tube as shown in Figure 3.3B. A linear relation for the first 3 to 4 datapoints is observed; however, beyond this point, the data deviate from linearity. The observation of a linear region for the uptake of the first deuteriums has been observed in studies of positively-charged protein ions and has been ascribed to fastexchanging sites near the charge sites.[27, 28] Using a linear fit to the first data points 


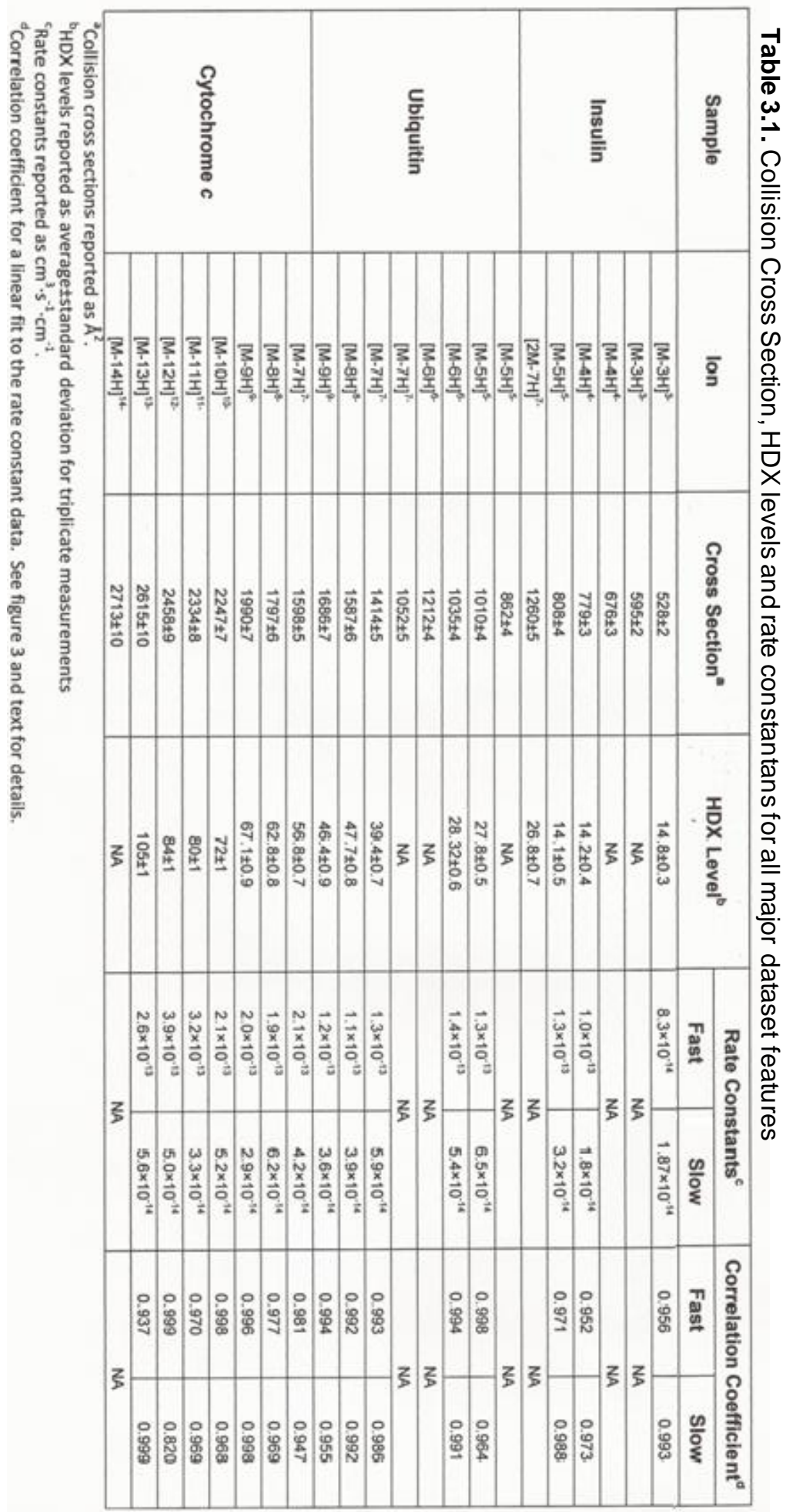


(Figure 3.3B) allows the determination of an HDX rate constant for the fast-exchanging sites. For the $[\mathrm{M}-5 \mathrm{H}]^{5-}$ ions, this rate constant is determined to be $1.3 \times 10^{-13} \mathrm{~cm}^{3} \cdot \mathrm{s}^{-}$ ${ }^{1}$. molecule $^{-1}$. The data in Figure 3.3B also suggest that some hydrogens exchange at a slower rate. Using the remaining data points up to those representing complete exchange, a second linear correlation provides a slower rate constant of $6.5 \times 10^{-14}$ $\mathrm{cm}^{3} \cdot \mathrm{s}^{-1} \cdot$ molecule $^{-1}$.

The maximum HDX levels and rate constants for all dominant ion conformations are listed in Table 3.1. For many conformations, both faster- and slower-exchanging hydrogens can be determined and therefore both rate constants are reported in Table 3.1. Comparatively, the reported rate constants are similar to those reported previously for positively-charged protein ions exposed to $\mathrm{D}_{2} \mathrm{O}$ in a drift tube.[27, 28] This determination is of interest indicating that a similar charge-mediated exchange process (see below) could account for the observed HDX.

\subsubsection{Anion HDX levels}

Figure 3.4 shows the maximum HDX levels of the predominant ion conformers (Table 3.1) from bovine insulin, globular proteins ubiquitin and cytochrome $c$. Interestingly, insulin exchange appear similar across the charge state distribution (-3 to 5). As mentioned above, this may be attributed to the 4 disulfide bonds that act to restrict the protein for forming extended conformation. Insulin CCS values are similar across the proteins charge state distribution (Table 3.1). This may account for similar HDX uptake values shown in Figure 3.4. For ubiquitin, the HDX levels for the $[\mathrm{M}-5 \mathrm{H}]^{5-}$ and $[\mathrm{M}-6 \mathrm{H}]^{6-}$ ions increase only slightly. Notably, the two ions have similar collision 


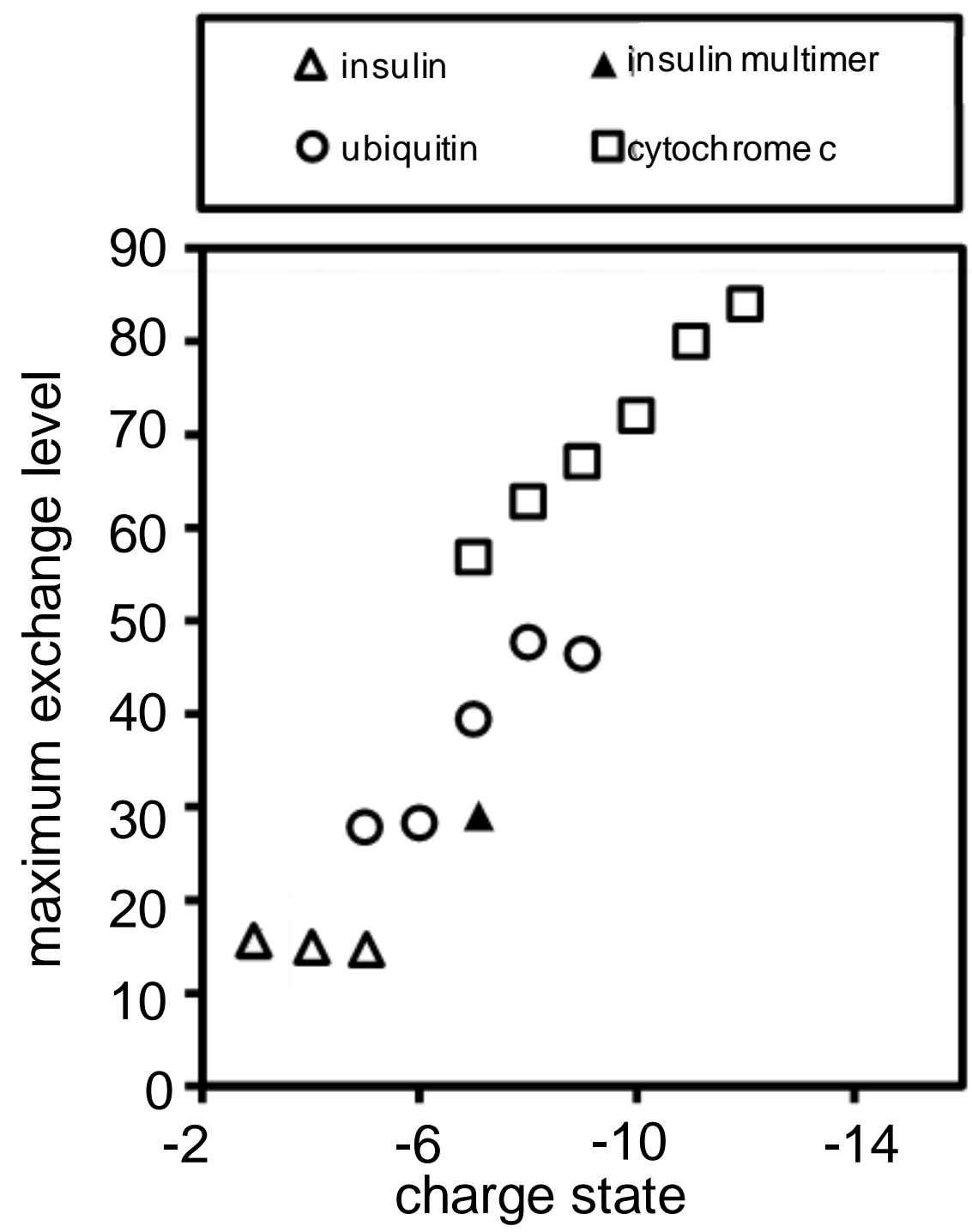

Figure 3.4. Plots of the maximum HDX levels for the dominant protein conformers. Diamond, circle and square symbols represent negatively-charged ions of the model peptide, bovine insulin, ubiquitin, and equine cytochrome c, respectively. Solid and open symbols represent monomeric and multimeric species, respectively. The number of individual monomers within multimeric species precedes the symbol.

cross sections (1010 $\AA^{2}$ and $1035 \AA^{2}$, respectively). The HDX level of the $[\mathrm{M}-7 \mathrm{H}]^{7-}$ ions increases by nearly $\sim 40 \%$ relative to that of the $[\mathrm{M}-6 \mathrm{H}]^{-6}$ ions. Similarly, the collision cross section for these ions is observed to be $\sim 37 \%$ larger than that of the more compact $[\mathrm{M}-6 \mathrm{H}]^{6-}$ ions representing the largest conformational change between any ions observed in the charge state distribution for ubiquitin. An increase in HDX level 
$(\sim 20 \%)$ is also observed for the $[\mathrm{M}-8 \mathrm{H}]^{8-}$ ions relative to that of the $[\mathrm{M}-7 \mathrm{H}]^{7-}$ ions while the change in collision cross section is smaller $(\sim 12 \%)$. The HDX levels of the $[\mathrm{M}-8 \mathrm{H}]^{8-}$ and $[\mathrm{M}-9 \mathrm{H}]^{9-}$ ions are similar and the latter ions exhibit only a slightly larger collision cross section ( 6\%). In general, the collision cross section data show a transition for dominant dataset features going from compact conformations for the $[\mathrm{M}-6 \mathrm{H}]^{6-}$ ions to more elongated conformations for the $[\mathrm{M}-8 \mathrm{H}]^{8-}$ ions. The HDX levels reflect this transition as well; the more elongated species are observed to have greater deuterium incorporation.

Cytochrome $c$ is the largest biomolecule examined in this study. Figure 3.4 shows the HDX levels for the ion conformations of cytochrome $c$. Overall, higher charge states are associated with increased HDX levels across the distribution and deuterium exchange proceeds in a nearly linear relationship with respect to increasing charge state. For example, exchange levels increase in a uniform manner from the $[\mathrm{M}-8 \mathrm{H}]^{8-}$ ions to the $[\mathrm{M}-12 \mathrm{H}]^{12-}$ ions and correspond to an overall increase of $\sim 50 \%(\sim 57$ to $\sim 85)$. Collision cross sections increase by a nearly identical 53\% (1599 $\AA^{2}$ to $\left.2459 \AA^{2}\right)$ across this charge state range.

\subsubsection{HDX Kinetics of Deprotonated Peptides and Proteins}

Table 3.1 lists fast- and slow-exchanging rate constants for the various ion conformers. As mentioned above, for many ions, two rate constants have been determined. On average, the larger rate constants are $\sim 6$ times larger than the smaller rate constants. Of the larger rate constants, the fastest $\left(\mathrm{k}=6.4 \times 10^{-13} \mathrm{~cm}^{3} \cdot \mathrm{s}^{-1} \cdot \mathrm{molecule}^{-}\right.$ $\left.{ }^{1}\right)$ and slowest $\left(\mathrm{k}=3.9 \times 10^{-13} \mathrm{~cm}^{3} \cdot \mathrm{s}^{-1} \cdot \mathrm{molecule}^{-1}\right)$ exchanging hydrogens are observed for $[\mathrm{M}-11 \mathrm{H}]^{11-}$ ions from cytochrome $\mathrm{c}$ and bovine insulin, respectively. For the smaller 
rate constants the fastest $\left(\mathrm{k}=6.5 \times 10^{-14} \mathrm{~cm}^{3} \cdot \mathrm{s}^{-1} \cdot\right.$ molecule $\left.^{-1}\right)$ and slowest $\left(\mathrm{k}=1.8 \times 10^{-14}\right.$ $\mathrm{cm}^{3} \cdot \mathrm{s}^{-1} \cdot$ molecule ${ }^{-1}$ ) exchanging hydrogens are observed for $[\mathrm{M}-5 \mathrm{H}]^{5-}$ ubiquitin and $[\mathrm{M}-$ $4 \mathrm{H}]^{4-}$ insulin ions, respectively.

\subsubsection{Structural Implications of the HDX Results}

To discuss the IMS and HDX information with regard to ion structure, it is useful to consider the process of HDX for negative ions. Although a mechanism for HDX with peptide anions and $\mathrm{D}_{2} \mathrm{O}$ has not been presented in the literature, studies with other anion systems suggest the possibility of a relay mechanism.[26, 69, 70] For peptides, the exchange would occur via a long-lived reaction intermediate involving interaction of the $\mathrm{D}_{2} \mathrm{O}$ molecule at the deprotonated site as well as a less acidic site (e.g., the neutral carboxylic acid moiety of a neighboring acidic amino acid residue). Exchange would proceed with deuterium transfer to the charge site and abstraction of the proton from the less acidic site. In this regard, the accessibility of a given hetero-atom site could be described with regard to its accessibility or proximity to a charge site as described previously for positively charged ions.[23, 27] An additional factor would be the conformational flexibility of the specific ions; $[70,71]$ that is, molecular motions that can position charge sites within an accessible range of exchange sites should also be considered. Finally, accessibility of exchange sites to collision events is also required. Further discussion of structural interpretation of the data is presented with respect to such processes.

The insulin ions exhibit the smallest HDX levels and the slowest exchange rates. The observation of decreased HDX levels for ions formed from proteins with disulfide bonds has been reported previously for positively charged ions.[71] As before, it is 
proposed that conformational rigidity of insulin ions results in the decreased exchange. That is, limitations in molecular motion result in decreased access to exchange sites by charge sites. For ubiquitin ions, conformational rigidity and accessibility to $\mathrm{D}_{2} \mathrm{O}$ can explain the observed HDX levels. For example, the compact nature of the $[\mathrm{M}-5 \mathrm{H}]^{5-}$ and $[\mathrm{M}-6 \mathrm{H}]^{6-}$ ions may prevent access of specific exchange hydrogens to $\mathrm{D}_{2} \mathrm{O}$; protection by compact structures for positively-charged cytochrome $c$ ions has been presented previously.[28] It is also noted that the compact nature can also provide conformational rigidity as demonstrated in previous molecular modeling studies.[27] For the more elongated, higher charge states of ubiquitin, the increased accessibility of exchange sites to $\mathrm{D}_{2} \mathrm{O}$ and increased conformational flexibility could account for the increased HDX levels. Finally, for the multiply-charged cytochrome $c$ ions, the relatively elongated nature of the ions would also expose more exchange sites to the $\mathrm{D}_{2} \mathrm{O}$ and the charge sites. The exposure to charge sites would increase with increasing charge and result in the observed higher HDX levels of these ions.

\subsubsection{Exchange Efficiency and the Protein Fold-Ubiquitin}

The discussion above of the maximum HDX levels for ubiquitin ion conformers demonstrates that HDX levels correlate to a conformational transformation occurring for the $[\mathrm{M}-6 \mathrm{H}]^{6-}$ and $[\mathrm{M}-7 \mathrm{H}]^{7-}$ ions. However, these maximum HDX levels do not reveal the degree to which increased exchange can be ascribed to protein fold versus an increase in the number of charge sites. To investigate this issue, HDX levels were recorded for compact and more elongated species for ions of both charge states at reduced $D_{2} \mathrm{O}$ pressures (0.04 Torr). The lower pressure was utilized to ensure sufficient signal of lower abundance conformers while at the same time determining differences in the 


\section{0}

\section{ubiquitin $[\mathrm{M}-6 \mathrm{H}]^{6-}$}

\section{5}

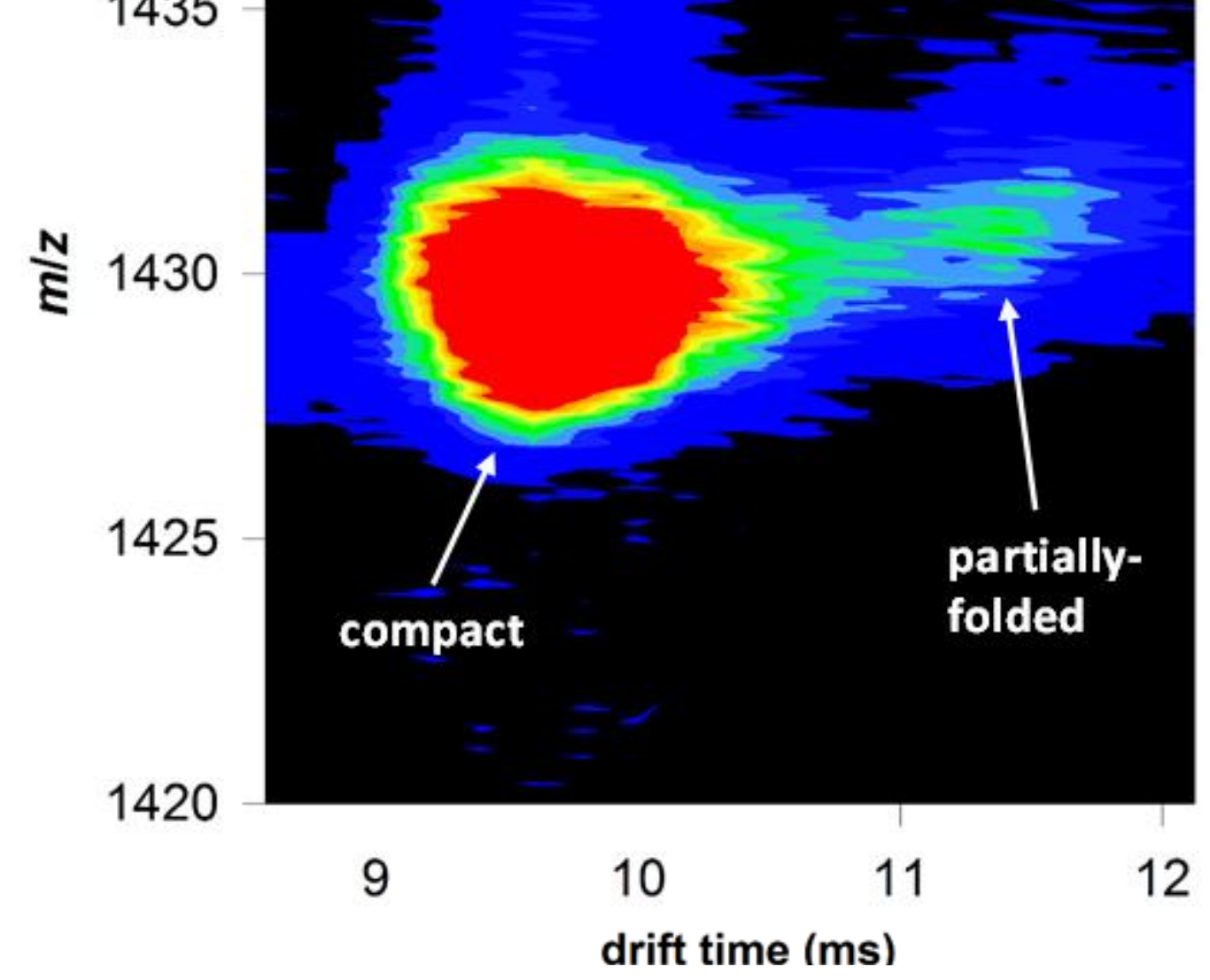

Figure 3.5.. Two-dimensional, $t_{D}(\mathrm{~m} / \mathrm{z})$ heat plot of the $[\mathrm{M}-6 \mathrm{H}] 6$ - charge state of ubiquitin after gas phase HDX with D2O at a partial pressure of 0.04 torr. Compact and partially folded anionic species are observed and are observed to exchange different amounts of deuterium (see text for details).

levels of rapidly exchanging hydrogens (see Figure 3.3). Figure 3.5 shows an expanded region of a $t_{D}, \mathrm{~m} / \mathrm{z}$ distribution for ubiquitin ions centered on compact and more elongated states of $[\mathrm{M}-6 \mathrm{H}]^{6-}$ conformers. On average, for the rapidly exchanging hydrogens, the more elongated conformer exhibits a $\mathrm{m} / \mathrm{z}$ value that is $\sim 1.0$ unit higher than the more compact conformer indicating an increased level of exchange corresponding to $\sim 6$ deuteriums. This same increase is also observed for more 
elongated $[\mathrm{M}-7 \mathrm{H}]^{7-}$ ions when compared with more compact species of the same charge. These observations suggest that roughly half of the increase in deuterium incorporation ( 11 deuteriums) from the compact $[\mathrm{M}-6 \mathrm{H}]^{6-}$ ions to the $[\mathrm{M}-7 \mathrm{H}]^{7-}$ ions can be attributed to the protein fold while the remaining difference can be accounted for by the addition of a charge site.

A more subtle contribution of exchange site protection provided by protein fold (i.e., locating exchange sites in interior regions) may be explained upon examining exchange efficiency. Exchange efficiency can be represented as the ratio of ion collision cross section to maximum HDX level. Figure 3.6 shows these ratios for all ions for which maximum HDX levels could be determined. A comparison of values determined for ubiquitin ions with those of cytochrome $\mathrm{c}$ ions suggests that the former ions exhibit slightly increased protection due to protein fold. That is, lower exchange efficiency is observed for the ubiquitin ions even though, on average, they exhibit a higher ( 14\%) charge site density. This lower exchange efficiency could result from less accessibility to buried exchange sites. Insulin ions yield an exchange efficiency that is similar to the monomeric insulin ions suggesting that the conformational rigidity afforded by the disulfide bonds, also affects the maximum exchange level that can be achieved by these ions.

Having described the HDX levels and rates with regard to protein fold and conformational flexibility, it is instructive to present an alternative explanation for the experimental results. Over the last two decades, seminal research has indicated a propensity for gas-phase peptide and protein ions as well as ion complexes to form salt bridges in the gas-phase. Arguably, such salt-bridge structures impose a degree of 

$\Delta$ insulin
$\Delta$ insulin multimer
ubiquitin

Deytochrome c

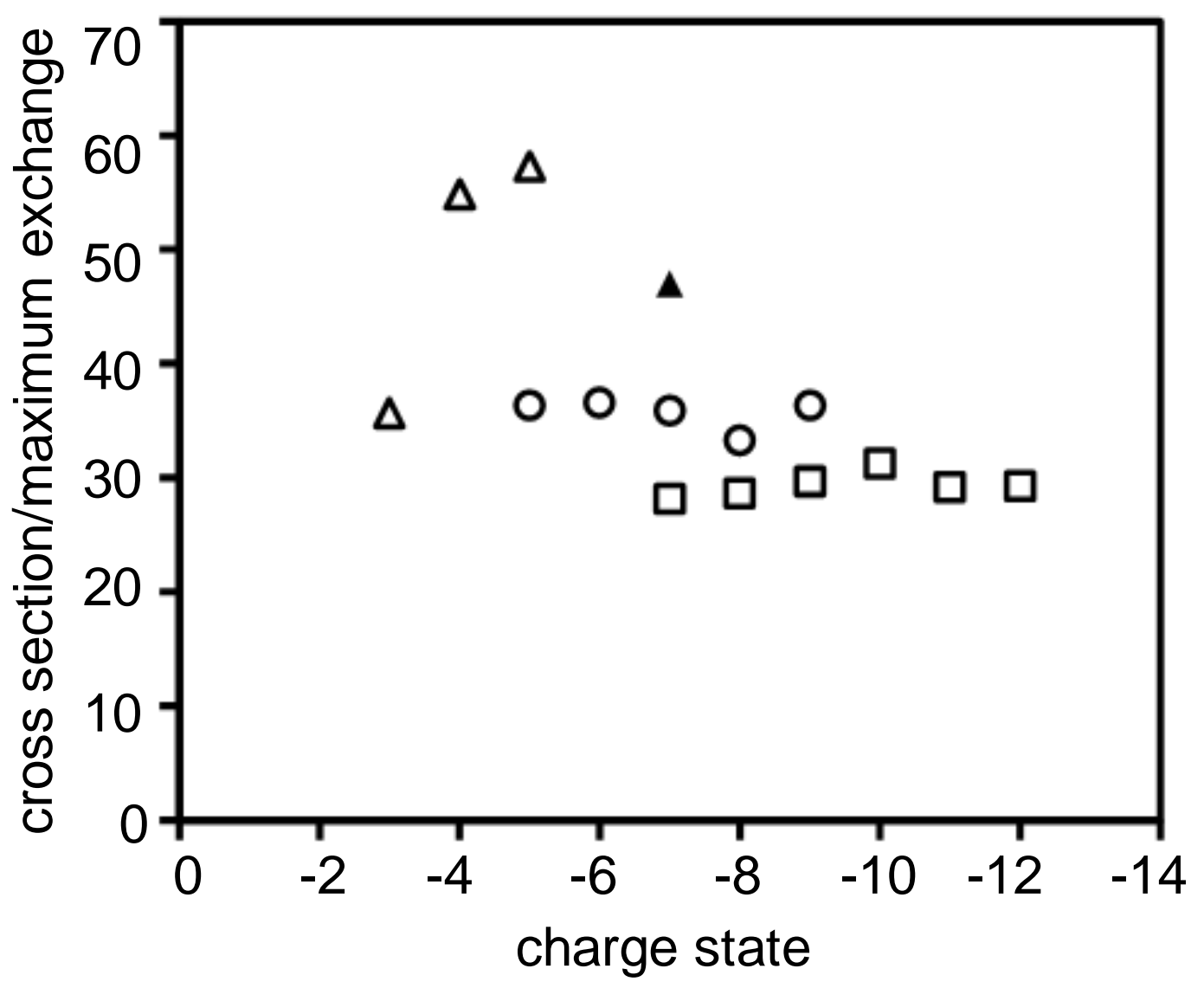

Figure 3.6. Plots of cross section/maximum HDX levels for the dominant protein conformers. Diamond, circle and square symbols represent negatively-charged ions of the model peptide, bovine insulin, ubiquitin, and equine cytochrome c, respectively. Solid and open symbols represent monomeric and multimeric species, respectively. The number of individual monomers within multimeric species precedes the symbol.

conformational inflexibility which could prevent exchange even at sites more proximal to charge sites. Here the argument of the necessity of charge site and exchange site proximity has been advanced based on the explanations provided for the observed maximum exchange levels and exchange efficiencies. The uniform increase in exchange levels for extended conformations of cytochrome $\mathrm{c}$ with additional charge as 
well as the differences in efficiencies between ubiquitin and cytochrome c suggest deuterium uptake near charge sites. This occurs even though acidic and basic residues are observed in flanking regions of the peptide; that is, even with the possibility of forming ion conformers with salt-bridges, increased exchange is observed. That said, other factors associated with the fast- and slow-exchanging sites cannot be ruled out. Indeed the work presented here lays the foundation for testing different exchange models as initiated in recent peptide ion conformer studies.

\subsubsection{Experimental limitations.}

The combination of IMS with HDX-MS demonstrates a powerful approach for characterizing the ion structures of negatively-charged biomolecular ions. The use of non-ergodic ion fragmentation techniques with MS analysis has proven useful in the localization of HDX sites.[72, 73] More recently, IMS-HDX experiments have been coupled with ETD-MS to determine deuterium uptake sites for specific conformations of positively-charged ions for the model peptide described here.[74] The addition of a suitable, non-ergodic ion fragmentation technique with IMS-HDX characterization of specific peptide and protein anions would allow similar determinations for the specific peptide and protein ions presented here. The development of new ion fragmentation methods offers promise for these experiments.[75-79]

Previously, molecular dynamics simulations have been used with IMS-HDX-MS results to help explain the HDX levels observed for positively-charged peptide and protein ions.[23, 27] The recent experiments combining IMS-HDX and ETD-MS with molecular dynamics simulations suggest a need for a refinement of the previous models for deuterium incorporation (i.e., those considering only the distance between exchange 
sites and charge sites). The observation that many of exchangeable hydrogens on charge sites do not undergo exchange necessitated the consideration of other models to be explored by molecular dynamics simulations. Indeed a model that incorporated distances to the charge site as well as to less basic sites using computer-generated peptide ion structures provide a better fit to experimentally determined deuterium uptake by individual amino acid residues.[74] Extending the experimental capabilities to allow determination of deuterium uptake at individual residues and the development of models from molecular dynamics simulations to explain these uptake levels will be pursued in future studies.

\subsubsection{Conclusions}

The HDX characteristics of negatively-charged ion conformers for peptides and proteins have been studied with IMS-MS techniques. Electrosprayed insulin ions exhibit the lowest HDX levels which can be attributed to decreased conformational flexibility resulting from the restraining disulfide bonds. This is further supported by the observation of the low exchange rates for these ions. HDX levels of larger proteins are observed to increase with charge state and ion size consistent with a charge-mediated exchange process. Of note, the HDX levels for ubiquitin ions scale with a conformational transition from more compact species to more elongated species. Based on comparisons to exchange protection for compact and elongated ion conformers, it is proposed that the exchange level transition results in part from protein fold exchange site protection and decreased access to charge sites for these ubiquitin ions. Finally, exchange efficiency comparisons suggest that ubiquitin ion conformers provide increased protection of interior sites relative to cytochrome $c$ ions. Together the 
results demonstrate the utility of the combined IMS and HDX conformational probes for obtaining structural information. Moreover, these studies lay the groundwork for powerful new approaches where additional tools including the incorporation of novel ion fragmentation techniques and the use of molecular dynamics simulations can be used to refine the structural information afforded by IMS-HDX measurements. 


\subsubsection{References}

1. Fenn, J.B., et al., ELECTROSPRAY IONIZATION FOR MASS-SPECTROMETRY OF LARGE BIOMOLECULES. Science, 1989. 246(4926): p. 64-71.

2. Hillenkamp, F., et al., MATRIX-ASSISTED LASER DESORPTION IONIZATION MASS-SPECTROMETRY OF BIOPOLYMERS. Analytical Chemistry, 1991. 63(24): p. A1193-A1202.

3. Tanaka, K., et al., Protein and polymer analyses up to $\mathrm{m} / \mathrm{z} 100000$ by laser ionization time-offlight mass spectrometry. Rapid Communications in Mass Spectrometry, 1988. 2(8): p. 151-153.

4. Loo, J.A., C.G. Edmonds, and R.D. Smith, Primary sequence information from intact proteins by electrospray ionization tandem mass spectrometry. Science, 1990. 248(4952): p. 201-4.

5. Cheng, X.H. and C. Fenselau, HYDROGEN-DEUTERIUM EXCHANGE OF MASS-SELECTED PEPTIDE IONS WITH ND3 IN A TANDEM SECTOR MASS-SPECTROMETER. International Journal of MasS Spectrometry and Ion Processes, 1992. 122: p. 109-119.

6. Suckau, D., et al., Coexisting stable conformations of gaseous protein ions. Proceedings of the National Academy of Sciences of the United States of America, 1993. 90(3): p. 790-793.

7. Covey, T. and D.J. Douglas, Collision cross sections for protein ions. J Am Soc Mass Spectrom, 1993. 4(8): p. 616-23.

8. Campbell, S., et al., Deuterium exchange reactions as a probe of biomolecule structure. Fundamental studies of cas phase $H / D$ exchange reactions of protonated glycine oligomers with $D 2 O, C D 3 O D, C D 3 C O 2 D$, and ND3. Journal of the American Chemical Society, 1995. 117(51): p. 12840-12854.

9. Clemmer, D.E., R.R. Hudgins, and M.F. Jarrold, Naked Protein Conformations: Cytochrome c in the Gas Phase. Journal of the American Chemical Society, 1995. 117(40): p. 10141-10142.

10. Meot-Ner, M., et al., Thermal decomposition kinetics of protonated peptides and peptide dimers, and comparison with surface-induced dissociation. Rapid Commun Mass Spectrom, 1995. 9(9): p. 829-36.

11. Kaltashov, I.A., D. Fabris, and C.C. Fenselau, ASSESSMENT OF GAS-PHASE BASICITIES OF PROTONATED PEPTIDES BY THE KINETIC METHOD. Journal of Physical Chemistry, 1995. 99(24): p. 10046-10051.

12. Williams, E.R., Proton transfer reactivity of large multiply charged ions. J Mass Spectrom, 1996. 31(8): p. 831-42.

13. Dongre, A.R., et al., Influence of peptide composition, gas-phase basicity, and chemical modification on fragmentation efficiency: Evidence for the mobile proton model. Journal of the American Chemical Society, 1996. 118(35): p. 8365-8374.

14. Schnier, P.D., et al., Blackbody infrared radiative dissociation of bradykinin and its analogues: energetics, dynamics, and evidence for salt-bridge structures in the gas phase. J Am Chem Soc, 1996. 118(30): p. 7178-89.

15. Green, M.K. and C.B. Lebrilla, Ion-molecule reactions as probes of gas-phase structures of peptides and proteins. Mass Spectrom Rev, 1997. 16(2): p. 53-71.

16. Rodriguez-Cruz, S.E., J.S. Klassen, and E.R. Williams, Hydration of Gas-Phase Gramicidin S (M + 2H)2+ lons Formed by Electrospray: The Transition From Solution to Gas-Phase Structure. Journal of the American Society for Mass Spectrometry, 1997. 8(5): p. 565-568.

17. Stephenson, J.L. and S.A. McLuckey, Gaseous protein cations are amphoteric. Journal of the American Chemical Society, 1997. 119(7): p. 1688-1696.

18. Javahery, G. and B. Thomson, A segmented radiofrequency-only quadrupole collision cell for measurements of ion collision cross section on a triple quadrupole mass spectrometer. Journal of the American Society for Mass Spectrometry, 1997. 8(7): p. 697-702. 
19. Dugourd, P., et al., High-resolution ion mobility measurements. Review of Scientific Instruments, 1997. 68(2): p. 1122-1129.

20. Valentine, S.J., A.E. Counterman, and D.E. Clemmer, Conformer-dependent proton-transfer reactions of ubiquitin ions. Journal of the American Society for Mass Spectrometry, 1997. 8(9): $p$. 954-961.

21. Wood, T.D., et al., GAS-PHASE FOLDING AND UNFOLDING OF CYTOCHROME-C CATIONS. Proceedings of the National Academy of Sciences of the United States of America, 1995. 92(7): p. 2451-2454.

22. Gard, E., et al., Gas-phase hydrogen/deuterium exchange as a molecular probe for the interaction of methanol and protonated peptides. J Am Soc Mass Spectrom, 1994. 5(7): p. 62331.

23. Wyttenbach, T. and M.T. Bowers, Gas phase conformations of biological molecules: The hydrogen/deuterium exchange mechanism. Journal of the American Society for Mass Spectrometry, 1999. 10(1): p. 9-14.

24. Freitas, M.A., et al., Gas-phase bovine ubiquitin cation conformations resolved by gas-phase hydrogen/deuterium exchange rate and extent. International Journal of Mass Spectrometry, 1999. 185: p. 565-575.

25. Wyttenbach, T., G. vonHelden, and M.T. Bowers, Gas-phase conformation of biological molecules: Bradykinin. Journal of the American Chemical Society, 1996. 118(35): p. 8355-8364.

26. Tian, Z., L. Lis, and S.R. Kass, Hydrogen-deuterium exchange and selective labeling of deprotonated amino acids and peptides in the gas phase. J Am Chem Soc, 2008. 130(1): p. 8-9.

27. Valentine, S.J. and D.E. Clemmer, Temperature-dependent $H / D$ exchange of compact and elongated cytochrome $c$ ions in the gas phase. Journal of the American Society for Mass Spectrometry, 2002. 13(5): p. 506-517.

28. Valentine, S.J. and D.E. Clemmer, $H / D$ exchange levels of shape-resolved cytochrome $c$ conformers in the gas phase. Journal of the American Chemical Society, 1997. 119(15): p. 35583566 .

29. Hoaglund, C.S., et al., Three-dimensional ion mobility TOFMS analysis of electrosprayed biomolecules. Analytical Chemistry, 1998. 70(11): p. 2236-2242.

30. Hoaglund, C.S., S.J. Valentine, and D.E. Clemmer, An ion trap interface for ESI-ion mobility experiments. Analytical Chemistry, 1997. 69(20): p. 4156-4161.

31. Valentine, S.J., S.L. Koeniger, and D.E. Clemmer, A split-field drift tube for separation and efficient fragmentation of biomolecular ions. Analytical Chemistry, 2003. 75(22): p. 6202-6208.

32. Merenbloom, S.I., et al., IMS-IMS and IMS-IMS-IMS/MS for separating peptide and protein fragment ions. Analytical Chemistry, 2006. 78(8): p. 2802-2809.

33. Wittmer, D., et al., ELECTROSPRAY-IONIZATION ION MOBILITY SPECTROMETRY. Analytical Chemistry, 1994. 66(14): p. 2348-2355.

34. Clemmer, D.E., R.R. Hudgins, and M.F. Jarrold, NAKED PROTEIN CONFORMATIONS CYTOCHROME-C IN THE GAS-PHASE. Journal of the American Chemical Society, 1995. 117(40): p. 10141-10142.

35. Hoadlund-Hyzer, C.S., J.W. Li, and D.E. Clemmer, Mobility labeling for parallel CID of ion mixtures. Analytical Chemistry, 2000. 72(13): p. 2737-2740.

36. Wu, C., et al., Electrospray ionization high-resolution ion mobility spectrometry - Mass spectrometry. Analytical Chemistry, 1998. 70(23): p. 4929-4938.

37. Gillig, K.J., et al., Coupling high-pressure MALDI with ion mobility/orthogonal time-of flight mass spectrometry. Analytical Chemistry, 2000. 72(17): p. 3965-3971. 
38. Ruotolo, B.T., et al., Peak capacity of ion mobility mass spectrometry: Separation of peptides in helium buffer gas. Journal of Chromatography B-Analytical Technologies in the Biomedical and Life Sciences, 2002. 782(1-2): p. 385-392.

39. Clowers, B.H. and H.H. Hill, Mass analysis of mobility-selected ion populations using dual gate, ion mobility, quadrupole ion trap mass spectrometry. Analytical Chemistry, 2005. 77(18): p. 5877-5885.

40. Clowers, B.H., et al., Hadamard transform ion mobility spectrometry. Anal Chem, 2006. 78(1): $\mathrm{p}$. 44-51.

41. Gillig, K.J., et al., An electrostatic focusing ion guide for ion mobility-mass spectrometry. International Journal of Mass Spectrometry, 2004. 239(1): p. 43-49.

42. Kwasnik, M., et al., Performance, resolving power, and radial ion distributions of a prototype nanoelectrospray ionization resistive glass atmospheric pressure ion mobility spectrometer. Analytical Chemistry, 2007. 79(20): p. 7782-7791.

43. Kwasnik, M., J. Caramore, and F.M. Fernandez, Digitally-Multiplexed Nanoelectrospray Ionization Atmospheric Pressure Drift Tube Ion Mobility Spectrometry. Analytical Chemistry, 2009. 81(4): p. 1587-1594.

44. Kurulugama, R.T., et al., Overtone Mobility Spectrometry: Part 1. Experimental Observations. Journal of the American Society for Mass Spectrometry, 2009. 20(5): p. 729-737.

45. Merenbloom, S.I., et al., High-resolution ion cyclotron mobility spectrometry. Anal Chem, 2009. 81(4): p. 1482-7.

46. Valentine, S.J., et al., Overtone Mobility Spectrometry: Part 2. Theoretical Considerations of Resolving Power. Journal of the American Society for Mass Spectrometry, 2009. 20(5): p. 738750.

47. Revercomb, H.E. and E.A. Mason, THEORY OF PLASMA CHROMATOGRAPHY GASEOUS ELECTROPHORESIS - REVIEW. Analytical Chemistry, 1975. 47(7): p. 970-983.

48. Siems, W.F., et al., MEASURING THE RESOLVING POWER OF ION MOBILITY SPECTROMETERS. Analytical Chemistry, 1994. 66(23): p. 4195-4201.

49. Mason, E.A.M., E. W., Transport Properties of lons in Gases. 1988, New York: Wiley.

50. Shvartsburg, A.A. and M.F. Jarrold, An exact hard-spheres scattering model for the mobilities of polyatomic ions. Chemical Physics Letters, 1996. 261(1-2): p. 86-91.

51. Mesleh, M.F., et al., Structural Information from Ion Mobility Measurements: Effects of the Long-Range Potential. The Journal of Physical Chemistry, 1996. 100(40): p. 16082-16086.

52. Wyttenbach, T., et al., Effect of the long-range potential on ion mobility measurements. Journal of the American Society for Mass Spectrometry, 1997. 8(3): p. 275-282.

53. Harris, G.A. and F.M. Fernandez, Simulations and Experimental Investigation of Atmospheric Transport in an Ambient Metastable-Induced Chemical Ionization Source. Analytical Chemistry, 2009. 81(1): p. 322-329.

54. Kwasnik, M. and F.M. Fernandez, Theoretical and experimental study of the achievable separation power in resistive-glass atmospheric pressure ion mobility spectrometry. Rapid Communications in Mass Spectrometry, 2010. 24(13): p. 1911-1918.

55. Glaskin, R.S., S.J. Valentine, and D.E. Clemmer, A Scanning Frequency Mode for lon Cyclotron Mobility Spectrometry. Analytical Chemistry, 2010. 82(19): p. 8266-8271.

56. Valentine, S.J., R.T. Kurulugama, and D.E. Clemmer, Overtone Mobility Spectrometry: Part 3. On the Origin of Peaks. Journal of the American Society for Mass Spectrometry, 2011. 22(5): p. 804816.

57. Siems, W.F., L.A. Viehland, and H.H. Hill, Jr., Improved momentum-transfer theory for ion mobility. 1. Derivation of the fundamental equation. Anal Chem, 2012. 84(22): p. 9782-91. 
58. Stlouis, R.H. and H.H. Hill, ION MOBILITY SPECTROMETRY IN ANALYTICAL-CHEMISTRY. Critical Reviews in Analytical Chemistry, 1990. 21(5): p. 321-355.

59. Clemmer, D.E. and M.F. Jarrold, Ion mobility measurements and their applications to clusters and biomolecules. Journal of Mass Spectrometry, 1997. 32(6): p. 577-592.

60. Hoaglund-Hyzer, C.S., A.E. Counterman, and D.E. Clemmer, Anhydrous protein ions. Chemical Reviews, 1999. 99(10): p. 3037-3079.

61. Collins, D.C. and M.L. Lee, Developments in ion mobility spectrometry-mass spectrometry. Analytical and Bioanalytical Chemistry, 2002. 372(1): p. 66-73.

62. Bohrer, B.C., et al., Biomolecule Analysis by Ion Mobility Spectrometry, in Annual Review of Analytical Chemistry. 2008. p. 293-327.

63. Donohoe G. C.; Maleki, H.A., J. R.; Khakinejad, M.; Yi, J.; McBride, C.; Nurkiewicz, T. R.; Valentine, S. J., A New Ion Mobility-Linear Ion Trap Instrument for Complex Mixture Analysis. Anal. Chem., 2014. 86(16): p. 8.

64. Tang, K., et al., High-sensitivity ion mobility spectrometry/mass spectrometry using electrodynamic ion funnel interfaces. Analytical Chemistry, 2005. 77(10): p. 3330-3339.

65. Lee, S., et al., Extracted fragment ion mobility distributions: A new method for complex mixture analysis. International Journal of Mass Spectrometry, 2012. 309: p. 154-160.

66. Shelimov, K.B. and M.F. Jarrold, Conformations, unfolding, and refolding of apomyoglobin in vacuum: An activation barrier for gas-phase protein folding. Journal of the American Chemical Society, 1997. 119(13): p. 2987-2994.

67. Shelimov, K.B., et al., Protein structure in vacuo: Gas-phase confirmations of BPTI and cytochrome c. Journal of the American Chemical Society, 1997. 119(9): p. 2240-2248.

68. Valentine, S.J., et al., Disulfide-intact and -reduced lysozyme in the gas phase: Conformations and pathways of folding and unfolding. Journal of Physical Chemistry B, 1997. 101(19): p. 38913900.

69. Chan, S. and C.G. Enke, Mechanistic study of hydrogen/deuterium exchange between [M - 1]ions of chlorinated benzenes and D2O or ND3. Journal of the American Society for Mass Spectrometry, 1994. 5(4): p. 282-291.

70. Freitas, M.A., et al., Gas-phase RNA and DNA ions. 1. H/D exchange of the M-H (-) anions of nucleoside 5 '-monophosphates (GMP, dGMP, AMP, dAMP, CMP, dCMP, UMP, dTMP), ribose 5monophosphate, and 2-deoxyribose 5-monophosphate with D2O and D2S. Journal of the American Chemical Society, 1998. 120(39): p. 10187-10193.

71. Evans, S.E., N. Lueck, and E.M. Marzluff, Gas phase hydrogen/deuterium exchange of proteins in an ion trap mass spectrometer. International Journal of Mass Spectrometry, 2003. 222(1-3): p. 175-187.

72. Rand, K.D., et al., Site-Specific Analysis of Gas-Phase Hydrogen/Deuterium Exchange of Peptides and Proteins by Electron Transfer Dissociation. Analytical Chemistry, 2012. 84(4): p. 1931-1940.

73. Rand, K.D., et al., ETD in a Traveling Wave Ion Guide at Tuned Z-Spray lon Source Conditions Allows for Site-Specific Hydrogen/Deuterium Exchange Measurements. Journal of the American Society for Mass Spectrometry, 2011. 22(10): p. 1784-1793.

74. Khakinejad, M.M., H.; Arndt, J. R.; Donohoe, G. C.; Valentine, S. J., Combining lon Mobility Spectrometry with Hydrogen-Deuterium Exchange and Top-Down MS for Peptide Ion Structure Analysis. J. Am. Soc. Mass Spectrom. (submitted), 2014.

75. Swaney, D.L., et al., Supplemental activation method for high-efficiency electron-transfer dissociation of doubly protonated peptide precursors. Anal Chem, 2007. 79(2): p. 477-85.

76. Yoo, H.J., et al., Negative-ion electron capture dissociation: radical-driven fragmentation of charge-increased gaseous peptide anions. J Am Chem Soc, 2011. 133(42): p. 16790-3. 
77. Cook, S.L., et al., Comparison of CID, ETD and metastable atom-activated dissociation (MAD) of doubly and triply charged phosphorylated tau peptides. Journal of Mass Spectrometry, 2012. 47(6): p. 786-794.

78. Cook, S.L. and G.P. Jackson, Metastable Atom-Activated Dissociation Mass Spectrometry of Phosphorylated and Sulfonated Peptides in Negative Ion Mode. Journal of the American Society for Mass Spectrometry, 2011. 22(6): p. 1088-1099.

79. Cook, S.L., O.L. Collin, and G.P. Jackson, Metastable atom-activated dissociation mass spectrometry: leucine/isoleucine differentiation and ring cleavage of proline residues. Journal of Mass Spectrometry, 2009. 44(8): p. 1211-1223. 


\section{Online Deuterium Hydrogen Exchange and Protein Digestion Coupled with Ion}

Mobility Spectrometry and Tandem Mass Spectrometry ${ }^{1}$

${ }^{1}$ Reprinted in part with permission from Analytical Chemistry: Online Deuterium Hydrogen Exchange and Protein Digestion Coupled with lon Mobility Spectrometry and Tandem Mass Spectrometry. Gregory C. Donohoe, James R. Arndt, Stephen J. Valentine.. Anal. Chem., 2015, 87 (10), 5247-5254.

\subsection{Introduction: Deuterium Hydrogen Exchange and IMS - MS}

Hydrogen deuterium exchange-mass spectrometry (HDX-MS) is an isotopic labeling strategy used for the evaluation of protein structure,[1-3] folding dynamics[1, 2, 4-11] and as a method to characterize protein-protein and protein-drug interactions in the biopharmaceutical sector.[3, 9, 12] Many HDX-MS experiments use high performance liquid chromatography (LC) consisting of an immobilized pepsin column for online digestion followed by trapping and reversed phase separation of peptic fragments that are subsequently mass analyzed.[13,14] Using this "bottom-up" approach, deuterium incorporation at backbone amide locations can be evaluated on a per-peptide basis. Despite much success, $[1,4,15]$ bottom-up HDX-MS is limited by spatial resolution that is dependent upon the number of overlapping peptide fragments.

Although the techniques have been commonly accepted and highly successfully, the remaining difficulty regarding HDX-LC-MS and MS/MS experiments is mitigating backexchange before MS analysis. HDX-LC-MS methods require specialized cooling apparatuses $(0 \mathrm{C})$, low $\mathrm{pH}(\sim 2.5)$ mobile phases, high flow rates and fast gradients ( 10 minutes or less) to minimize backexcahnge.[16] Because peptide separation is generally based on hydrophobicitiy, a particular problem arises in the higher rate of backexchange as certain peptides adopt altered solution conformations as they partition into the $C_{18}$ stationary phase.[17] Other studies have shown that the 
solid support contained within immobilized pepsin columns can promote significantly high backexchange.[18] The relatively fast gradients can also create differences in retention times requiring data alignment algorithms for quantitative and qualitative comparisons between datasets.[19] Additionally, because the LC step must be performed quickly, co-eluting peptides can reduce ionization efficiency. This is particularly problematic with data dependant MS/MS which only samples the highest intensity precursor ions (top 5) for fragmentation per-elution-window. Peptide ion sensitivity is further reduced using non-activating ion transfer parameters to mitigate hydrogen/deuterium scrambling in the gas phase. For LC-MS/MS analysis, the compilation of these inherent difficulties may result in inadequate isotopic distributions of product ions from the analysis.

HDX studies have used commercial traveling wave ion mobility (TWIM) devices coupled with Time-of-flight mass analyzers for bottom-up peptide studies.[20, 21] In the work provided here, a low-pressure drift tube coupled to an LTQ Velos mass spectrometer (Thermo Scientific, CA) is used for per-residue deuterium analysis by ETD. It is further noted that TWIM instruments contain an ETD cell located before the mobility region,[22-24] meaning that the mobility separation is not utilized to distinguish precursor ion conformers. Thus, an advantage demonstrated in this work is the ability of drift tube IMS (DTIMS) to mobility separate ions before ETD without being subject to ion heating that would promote deuterium scrambling. That is, ions that traverse the low pressure drift tubes are thermolized, while TWIM instruments are shown to promote ion heating of mobility transferred ions exhibiting temperatures from $\sim 400 \mathrm{~K}$ to $\sim 7000 \mathrm{~K}$.[2527] Although these temperatures are dependent on the TWIM wave speed and wave 
height as well as the trapping partial pressure compositions, other studies have shown that heating processes promote deuterium scrambling.[28, 29]

A goal of this proof-of-principle work is to demonstrate the replacement of the LC with a DTIMS instrumental platform for the evaluation of protein secondary structures. A continuous online deuterium hydrogen exchange (DHX) and pepsin digestion (PD) method preformed prior to the IMS separation is demonstrated. DHX proceeds under exchange-out conditions,[30] where fully deuterated protein is exposed to $\mathrm{H}_{2} \mathrm{O}$. The protein exchanges deuteriums in unstructured regions faster than sites held in $\mathrm{H}$ bonding networks.[30] The DHX-PD process takes $\sim 60$ seconds and only requires micro-cross assemblies and fused silica capillary, similarly fashioned to a design described for the characterization of the iron-saturated $\mathrm{N}$-lobe of human serum transferrin.[31] The rapid DHX time ( 32 seconds) in this study is used to backwash fast exchanging heteroatoms for a qualitative view of secondary structural elements.

The mobility measurement provides the capability to match, via extracted ion drift time distributions (XIDTDs)[32], labeled peptic peptide ions with the unlabeled peptide ions. Because the unlabeled ions were first identified using collision induced dissociation (CID), the technique transfers identification to deuterated peptides, allowing a straightforward deuterium retention evaluation. Due to the high reproducibility of the gas phase separation, XIDTD matching is performed without the need for dataset alignment. Another advantage of the online method is the ability to select a specific mobility range for MS and MS/MS analysis. Because the online system is continuous and is neither elution window nor intensity limited (as with data-dependent LC-MS/MS analysis), ETD can be performed on precursor ions over several minutes to produce 
statistically adequate isotopic distributions of product ions. Lastly, this work presents the first solution-based deuterium exchange study using a low-pressure, linear-field IMS coupled with ion trapping MS-ETD.

\subsection{Methods and Materials}

\subsubsection{Sample Preparation}

Ubiquitin (bovine erythrocytes, 98\%) and lyophilized pepsin (porcine, 3200-4500 units/mg protein) were purchased from Sigma-Aldrich (St. Louis, MO) and used without further purification. Deuterium oxide (99.9\%), formic acid (99\%) and ammonium acetate were purchased from Sigma-Aldrich (St. Louis, MO). The peptide KKDDDDDIIKIIK (90.6\%) was purchased from Genscript (Piscataway, NJ, USA) and used without further purification. This peptide was used as a HDX or deuterium scrambling control (see below).

\subsubsection{On - Line $D H X-P D-I M S$ System}

. Ubiqutin Studies. Ubiquitin $(0.5 \mathrm{mg})$ was added to $1.0 \mathrm{~mL}$ of $\mathrm{D}_{2} \mathrm{O}(99.9 \%)$ containing ammonium acetate $(10 \mathrm{mM})$. The solution was incubated $\sim 40$ hours at room temperature. Note the 40 hour time period was utilized for the first of three replicate measurements. Subsequent replicate measurements were performed on successive days. Pepsin solutions were prepared by adding lyophilized powder $(0.75 \mathrm{mg})$ to $1.0 \mathrm{~mL}$ $18 \mathrm{M} \Omega \mathrm{H}_{2} \mathrm{O}$ (6\% formic acid v:v) $\mathrm{pH} \sim 2.0$. A schematic of the online system is presented in Figure 1.1 in the Supplementary Information section (Figure S1). Briefly, the DHX reaction and subsequent quenching with simultaneous digestion was performed using three micro-cross assemblies (Upchurch Scientific Inc, Oak Harbor, Wa) connected with fused-silica capillary (360 $\mu$ m o.d. $\times 50 \mu \mathrm{m}$ i.d). Separate $500-\mu \mathrm{L}$ syringes (Hamilton, 
Reno, NV, USA) containing the deuterated ubiquitin solution and $18 \mathrm{M} \Omega \mathrm{H}_{2} \mathrm{O}$ were delivered to the first micro-cross assembly using high precision syringe pumps (KD scientific Holliston, MA,USA) at flow rates of $0.20 \mu \mathrm{L} \cdot \mathrm{min}^{-1}$ and $0.87 \mu \mathrm{L} \cdot \mathrm{min}^{-1}$

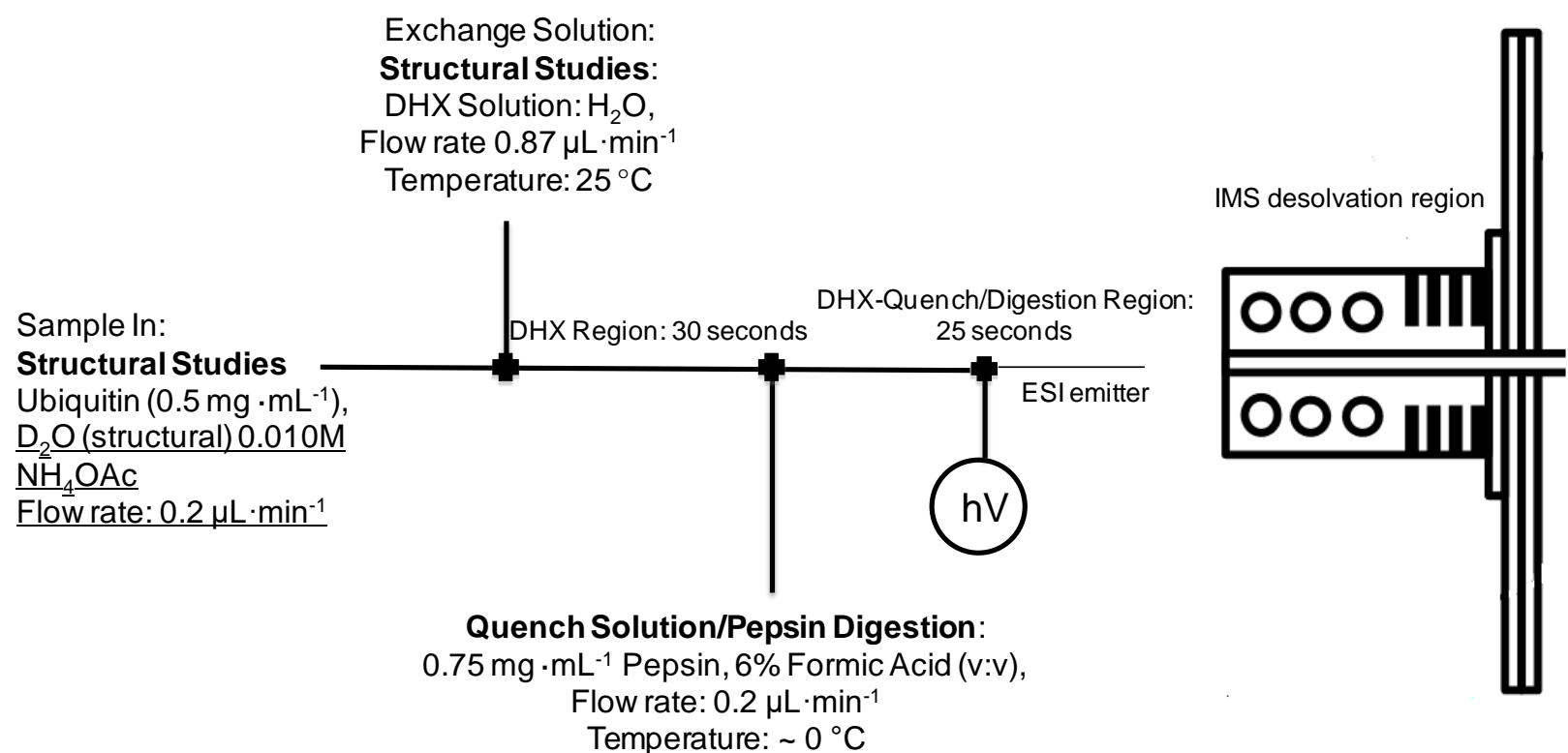

Figure 4.1.. Schematic representation of the on-line system for DHX-PD-ESI-IMS-MS analysis. High precesion syringe pumps were used to advance separate solutions simultaneously to mixing-tees (black crosses). At these locations, exchange reactions or digestion occurred along different lengths of capillary. The respective times for DHX and digestion are also indicated.

respectively. DHX of deuterated ubiquitin proceeded for $\sim 29.6$ seconds over a capillary length of $26.4 \mathrm{~cm}$. This DHX-setup and exchange time was similar to that reported in previous exchange-out experiments for the determination of site specific interactions of Huntingtin protein exon 1 aggregates.[33] A third 500- $\mu \mathrm{L}$ syringe containing ice-cooled pepsin solution ( $\mathrm{pH} \sim 2.0$ ) was introduced by a syringe pump to a second micro-cross assembly at a flow rate of $0.20 \mu \mathrm{L} \cdot \mathrm{min}^{-1}$. DHX quenching and protein digestion was preformed over a capillary length of $20.3 \mathrm{~cm}$. Peptic peptides entered into a third microcross assembly connected to a $2.5 \mathrm{~cm}$ pulled tip nano-ESI emitter $(360 \mu \mathrm{m}$ o.d. $\times 50 \mu \mathrm{m}$ i.d) giving a total digestion capillary length of $22.9 \mathrm{~cm}$ and a total digest time of $\sim 22.4$ 
seconds. Peptides were electrosprayed into the IMS instrument using a bias voltage of $\sim+1.9 \mathrm{kV}$ at a combined flow rate of $1.26 \mu \mathrm{L} \cdot \mathrm{min}^{-1}$. No direct heating of the source or desolvation region was applied. For unlabeled ubiquitin studies, the same protein concentrations, syringe flow rates and capillary lengths were used; however, ubiquitin was incubated in $18 \mathrm{M} \Omega \mathrm{H}_{2} \mathrm{O}$ containing $10 \mathrm{mM}$ ammonium acetate.

Here it is noted that no sample cleanup steps were performed. Although not necessary for the purified samples utilized in this study as demonstrated by the results presented below, because salt content may hinder ESI, sample cleanup for many protein samples may be required in order to take advantage of this direct infusion approach. That said, to some degree, the IMS separation can mitigate problems arising from noise produced by isobaric ions.[34]

\subsubsection{Scrambling Control On - Line System}

For the evaluation of scrambling, the peptide KKDDDDDIIKIIK $(0.5 \mathrm{mg})$ was added to $1.0-\mathrm{mL}$ of $\mathrm{D}_{2} \mathrm{O}(99.9 \%)$ containing $10 \mathrm{mM}$ ammonium acetate $(\mathrm{pH} \sim 7.0)$ and allowed to incubate for $\sim 24$ hours at $25^{\circ} \mathrm{C}$. The peptide contains a fast exchanging KKDDDDD sequence and a slow exchanging IIKIIK C-terminal region. For these studies, the DHX solution served as the quench solution in that it was cooled with an ice pack and acidified to a final $\mathrm{pH}$ of $\sim 2.5$. Flow rates for the peptide and $\mathrm{DHX}$ solutions were $0.20 \mu \mathrm{L} \cdot \mathrm{min}^{-1}$ and $1.00 \mu \mathrm{L} \cdot \mathrm{min}^{-1}$ respectively. Capillary lengths for $\mathrm{DHX}$ were the same as described for ubiquitin studies. It should be noted that the pepsin solution was not added to the online system, as the exchanged peptide must retain the intact sequence for scrambling determination. Instrumental parameters for ubiquitin 
deuteration studies were selected based upon the peptide exhibiting a $0 \%$ scrambling trend). $[28,29]$

\subsubsection{Optimized Parameters}

Instrumental parameters used to mitigate HD-scrambling can be found in Appendix 1. These parameters were determined based on the scrambling control peptide resulting in $0 \% \mathrm{HD}$-scrambling (see below).

\subsubsection{IMS-MS: Instrumental Operation}

IMS theory,[35-38] instrumentation,[39-45] and techniques[46-55] are described elsewhere in great detail. The IMS instrument contained a drift tube with dual ion funnels and two ion gates interfaced to a LTQ Velos (Thermo Electron, San Jose, Ca, USA) mass spectrometer that has been described previously.[56, 57] The LTQ Velos was outfitted with a commercial ETD source for the generation of fluoranthene radicals (Thermo Scientific, San Jose, Ca, USA). Figure 1.1 shows a schematic of the IMS-MS instrument with ETD capabilities and the locations of the ion funnels and gates within the drift tube. The source of the mass spectrometer was redesigned to incorporate a stacked-ring ion drift region by replacing the s-lens assembly (Figure 1.1). Briefly, ions were electrosprayed into the desolvation region and were focused through an "hourglass" electrodynamic ion funnel (F1 in Figure 1.1).[50, 58] The focused ions were periodically trapped at the first ion gate (G1 in Figure 1.1) and periodically pulsed into a 1-meter-long drift tube filled with He buffer gas ( 2.72 torr at $300 \mathrm{~K})$. Under the influence of a uniform electric field $\left(\sim 10 \mathrm{~V} \cdot \mathrm{cm}^{-1}\right)$, ions of different mobilities reached the second ion gate (G2 in Figure 1.1) at different times. lons of select drift times ( $\left.t_{D}\right)$ that passed through G2 were radially focused at the second ion funnel (F2)[50], and exited through a conductance-limiting aperture. The ion packet was subsequently transferred 
by quadrupole and octopole rf guides before entering into the high pressure linear ion trap followed by mass analysis in the lower pressure trap.

\subsubsection{Recording Two - Dimensional (2D) $t_{\underline{D}}(\mathrm{~m} / \mathrm{z})$ Distributions.}

Ion gates were constructed from lenses containing Ni grid (90\% transmittance mesh; Precision Eforming, Cortland, NY, USA). G1 was composed of a single gridded lens located directly behind the outlet of the hour-glass funnel. During ion accumulation, G1 was biased $\sim 45 \mathrm{~V}$ higher than the last lens of F1. For mobility measurements, an ion packet was pulsed into the drift tube by dropping the voltage at G1 for $150 \mu$ s every 20 ms. A $t_{D}$ was collected for mobility separated ions using a time-delayed voltage applied to the ion selection gate (G2). G2 consisted of two gridded lenses (Tyndall Geometry) spaced $\sim 0.30 \mathrm{~cm}$ apart. An attractive field $(2.5 \mathrm{~V})$ pulsed at specific times between the lenses enabled ions of a selected mobility to pass for mass analysis. Ions of all other mobilities were eliminated with a repulsive field applied between the lenses $(10.0 \mathrm{~V})$. The gate timing of G2 was initiated relative to the introductory ion pulse at G1. By scanning the delay time across the entire $t_{D}$ from 4.0 to $12.0 \mathrm{~ms}$, at a step resolution of $200 \mu \mathrm{s}, 41$ mobility-resolved mass spectra was collected. The introduction pulse at G1 and variable delay settings at G2 were controlled by 2 four-channel digital pulse generators (Stanford Research Systems, Sunnyvale, CA, USA).

\subsubsection{Mass Spectrometry Measurements}

Full mass spectra were collected for all ions by deactivating the two drift tube gates and setting the mass analyzer scan parameters over a $\mathrm{m} / \mathrm{z}$ range of 75 to 2000 and enabling the automatic gain control (AGC). In this operation, the drift tube acted to transfer all ions into the LTQ. The AGC threshold is set to $3 \times 10^{6}$ ions and a mass 
spectrum was collected. During $t_{D}$ distribution generation, the AGC was disabled and a trap injection time of $400 \mathrm{~ms}$ (4 microscans) was selected. All mobility-resolved mass spectra were collected and averaged over 0.5 minutes.

\subsubsection{CID-MS Experiments}

Tandem mass spectrometery (MS/MS) using CID was conducted on unlabeled ubiquitin peptic peptide ions by isolating a mobility selected ion $\mathrm{m} / \mathrm{z}$. For these experiments, an isolation window of \pm 3 Da relative to the centroid $\mathrm{m} / \mathrm{z}$ resulted in the ejection of all other ions within the ion trap. Selected precursor ions were collisionally activated with $\mathrm{He}$ gas at a normalized collision energy of $30.0 \%$ and an activation time of $10 \mathrm{~ms}$. Product ion spectra were collected for 1.0 minute.

\subsection{Identification of Precursor lons.}

For peptic peptide identification, the selected precursor $\mathrm{m} / \mathrm{z}$ values were converted to mass based on charge state. The masses were then derived to determine $[\mathrm{M}+\mathrm{H}]^{+}$ions. Using ExPaSY FindPept (http://web.expasy.org/findpept/) the unmodified 76 amino acid sequence of bovine ubiquitin was uploaded to perform in-silico digestion using pepsin at $\mathrm{pH}>2.0$. The program generated $[\mathrm{M}+\mathrm{H}]^{+}$ions at a mass tolerance of \pm 2.0 Da having unique amino acid lengths. The corresponding sequences with experimentally matching $\mathrm{m} / \mathrm{z}$ values were selected and entered into Protein Prospector (http://prospector.ucsf.edu/prospector/cgi-bin/msform.cgi?form=msproduct) for in-silico MS/MS fragmentation. Product ion spectra from the selected precursors collected from IMS-MS/MS experiments were compared to $y$ - and $b$ - fragment ions for identification.

\subsubsection{Deuterated Samples and ETD Analysis.}


The control peptide (KKDDDDDIIKIIK) served to evaluate the optimal settings applied to the source, ion optics, lenses and transfer voltages to mitigate HD scrambling. These settings were changed until the control peptide follows a $0 \%$ scrambling trend (see Supporting Information for parameters).[28, 29] Briefly, the F1 DC voltage (Figure 1.1) was set to $136.8 \mathrm{~V}$, the RF voltage at F2 was set to 136.8 volts peak-to-peak, the voltages on the ion guides were set to $300 \mathrm{~V}$ peak-to-peak and no direct temperature was applied to the ESI source. For ETD studies, a specific precursor $\mathrm{m} / \mathrm{z}$ was selected using a $\pm 8 \mathrm{Da}$ window relative to the centroid $\mathrm{m} / \mathrm{z}$ value. This wider selection window was used to confine the entire isotopic distribution. Furthermore, narrow selection windows have been shown to induce scrambling by resonance excitation.[28] ETD of selected $[\mathrm{M}+3 \mathrm{H}]^{3+}$ precursor ions proceeded by introduction of fluroanthene radicals into the LTQ for $100 \mathrm{~ms}$. To ensure adequate isotopic distribution statistics of product ions, the resulting spectra were collected for 1.0 minute.

\subsubsection{IMS-MS Data Sets.}

Two-dimensional IMS-MS datasets were generated by converting each $t_{\mathrm{D}}$ resolved mass spectrum (.RAW file) to a separate text file (.TXT file). Using software developed in-house, the $\mathrm{m} / \mathrm{z}$ values and intensities contained within this .TXT file at an associated $t_{D}$ were converted into a three-column array file containing $t_{D}, m / z$, and intensity information. Intensity filters were applied to the dataset for spectral clarity and the output can be used to create a 2D IMS-MS heat plot based on a logarithmic intensity scale. Here, each pixel contained a $t_{D}$ width of $200 \mu$ s and a height of 0.0833 Da.

4.2.4.4.Comparative XIDTDs for Identification. 
To generate XIDTDs[32], a separate in-house program was used to process the same three column array files ( $t_{\mathrm{D}}, \mathrm{m} / \mathrm{z}$, and intensity) by integrating intensity values across all $t_{D}$ windows within a user-defined $\mathrm{m} / \mathrm{z}$ range. The output comprised an integrated mobility distribution for specific $\mathrm{m} / \mathrm{z}$ values. All data were normalized to the most intense spectral feature within each XIDTD. XIDTDs were matched based on a pixel $t_{D}$ width of $200 \mu$ s and a pixel height of $0.0833 \mathrm{Da}$. No dataset alignment was utilized for XIDTD matching between undeuterated and deuterated digest samples.

\subsubsection{Per-Residue Deuterium Measurements.}

Product ions generated from ETD (triplicate measurements) were signal averaged over 1.0 minute. The resulting spectrum was converted in much the same manner as described above. Briefly, the .RAW file was converted into a two column .TXT file containing $\mathrm{m} / \mathrm{z}$ and associated intensity values. Using software developed inhouse, deuterium uptake was calculated from average $\mathrm{m} / \mathrm{z}$ determinations obtained by weighting the isotopic peaks according to their intensity values for each product ion. The output was a .TXT file containing the average $\mathrm{m} / \mathrm{z}$ values that were then subtracted from the undeuterated product ion average $\mathrm{m} / \mathrm{z}$ of the same charge state. The mass difference was reported as the amount of total deuterium retention for each fragment ion.

\subsection{Results and Discussion}

\subsubsection{Comparing Peptides from 2D IMS-MS Datasets.}

Figure 4.2A shows the two-dimensional $2 \mathrm{D} t_{D}(\mathrm{~m} / \mathrm{z})$ distribution of undeuterated peptic peptides resulting from online PD-IMS-MS. Spectral features are first observed 


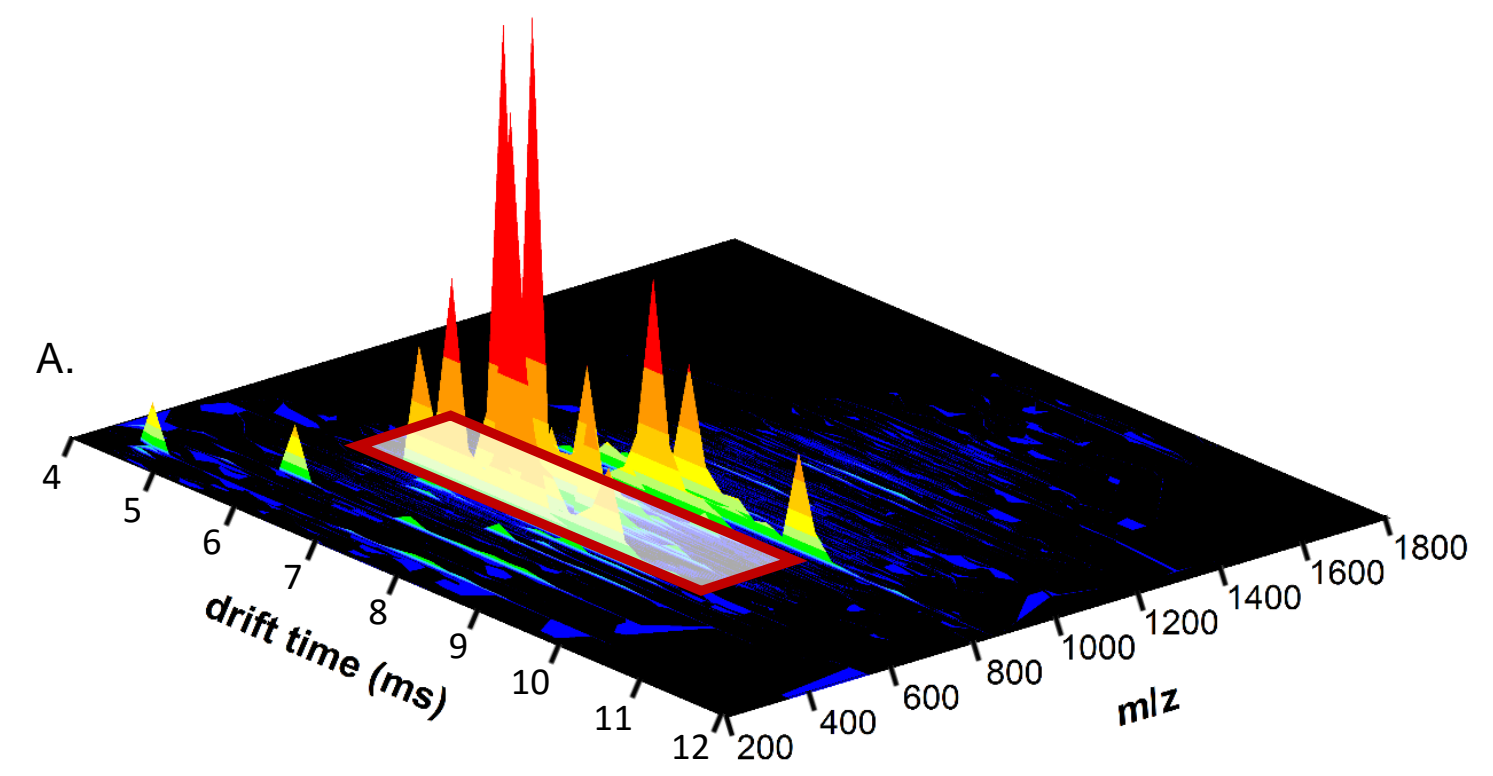

B.

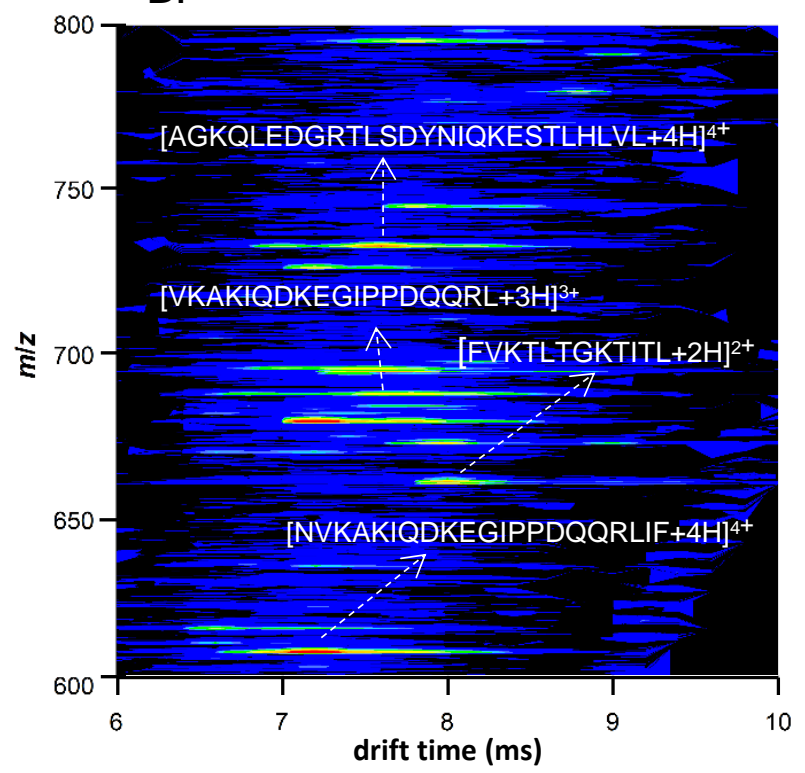

C.

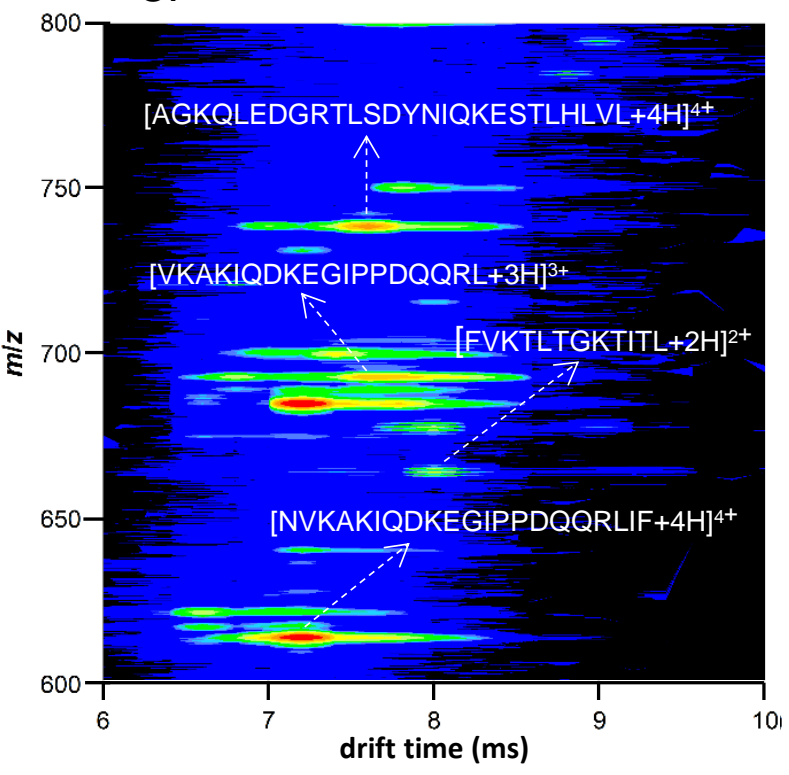

Figure 4.2 (A) Two-dimensional (2D) $t_{D}(\mathrm{~m} / \mathrm{z})$ raised-relief plot showing the resulting peptide ions generated from DHX-PD-IMS-MS of unlabeled ubiqutin. The dashed-line box represents the region from $t_{D} \sim 6$ to $\sim 10 \mathrm{~ms}$ and a $\mathrm{m} / \mathrm{z}$ range of 600 to 800 . This region is used for comparative purposes in Figures B and C. (B) $2 \mathrm{D} t_{D}(\mathrm{~m} / \mathrm{z})$ contour-plot of DHX-PD-IMS-MS analysis of unlabeled peptides extracted from the boxed region in Figure A. (C) $2 D t_{D}(\mathrm{~m} / z)$ contour-plot the DHX-PD-IMS-MS analysis of labeled peptides over the same $t_{D}(\mathrm{~m} / z)$ ranges as described above. The color maps for all plots are shown on a logarithmic scale (-2 to 3$)$ in order to show the low-intensity ions. 
at $\sim 4.6 \mathrm{~ms}$ and extend to $\sim 11.4 \mathrm{~ms}$ with the highest intensity peptide ions existing between a $\mathrm{m} / \mathrm{z}$ range of $\sim 550$ to $\sim 800$. Spectral intensities for mobility separated ions ranged from only a few counts to $\sim 4.2 \times 10^{3}$. Figure $4.2 \mathrm{~B}$ and $2 \mathrm{C}$ show the $2 \mathrm{D} t_{D}(\mathrm{~m} / \mathrm{z})$ contour depicting expanded regions of the dataset $\left(t_{D} 6.0 \mathrm{~ms}\right.$ to $10.0 \mathrm{~ms}$ over a $\mathrm{m} / \mathrm{z}$ range of 600 to 800 ) for undeuterated and deuterated peptides, respectively.

Collectively, Figures $4.2 \mathrm{~B}$ and $4.2 \mathrm{C}$ show similar spectral features that display similar drift times, but differ in average $\mathrm{m} / \mathrm{z}$ due to the incorporation of deuterium. It is also noted that deuterium retention broadens the isotopic distribution for given peptides (Figure 4.2C) and in some cases the isotopic envelopes are observed to overlap in the mass spectrum. For all sample sets, the fast proteolytic step generated larger peptides having sequence lengths between $\sim 4$ to $\sim 25$ residues and exhibit charge states from +1 to +4 . With respect to LC-MS approaches, these larger peptide sequences are problematic, as deuterium evaluation at the residue level is based on the number of overlapping peptides. Comparatively, the longer sequences contain a larger number of basic residues resulting in higher charge states more amenable for ETD fragmentation.

\subsubsection{Generating XIDTDs and Combining Collision Induced Dissociation (CID) for}

\section{Unlabeled Peptides Identification.}

A particular advantage of the IMS-MS measurement is the ability to generate an XIDTD for peptide ion analyzed. Additionally, XIDTDs allow a more exact comparison of features in IMS-MS plots (Figures 4.2B and 4.2C). For example, Figure 4.3A shows the 

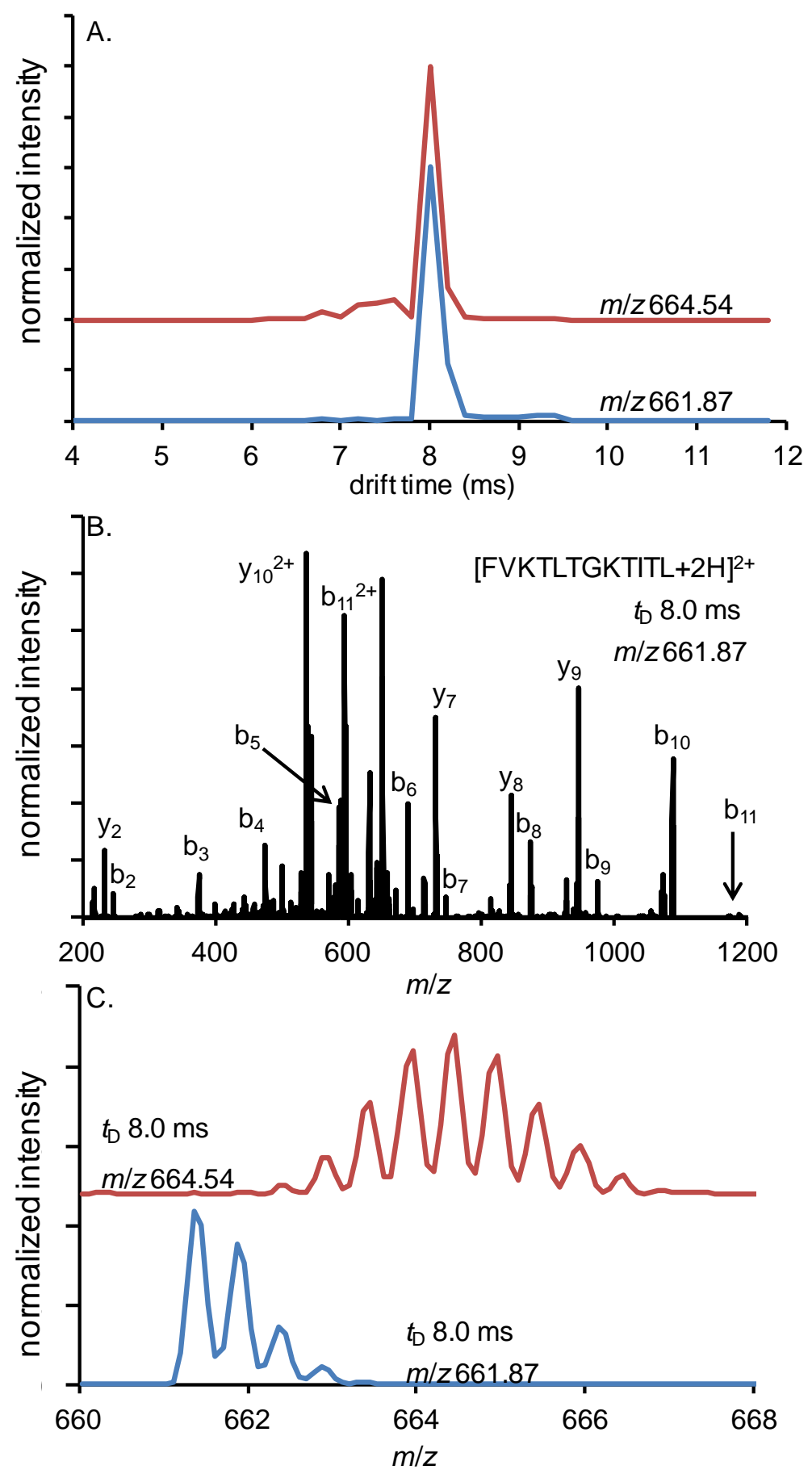

Figure 4.3. (A) XIDTD of the ion having an average $\mathrm{m} / \mathrm{z}$ of 661.87 from undeuterated ubiqutin (blue trace) and the overlayed XIDTD (red trace) of the respective deuterated ion (average $\mathrm{m} / \mathrm{z}$ of 664.54 ). All XIDTDs are normalized to the most intense spectral feature. (B) CID fragmentation spectrum obtained from the precursor ion having an averaged $\mathrm{m} / z$ of 661.87 . Several $b$ - and $y$-product ions are labeled that are consistent with the $[\text { FVKTLTGKTITL+2H] }]^{2+}$ peptide ion assignment. (C) Overlays of Isotopic distributions for the peptide ion of average $\mathrm{m} / \mathrm{z} 661.87$ (blue trace) and the same peptide ion of average $\mathrm{m} / \mathrm{z} 664.54$ (red trace) extracted from the respective mass spectrum collected at $t_{\mathrm{D}} \sim 8.0 \mathrm{~ms}$. 
XIDTD of an undeuterated ion having an average $\mathrm{m} / \mathrm{z}$ of 661.87 . The XIDTD shows a dominate ion conformer type with a maximum intensity at $\sim 8.0 \mathrm{~ms}$ for this given $[\mathrm{M}+2 \mathrm{H}]^{2+}$ peptide ion. To some degree, the XIDTD becomes a spectral-fingerprint that is unique ( $t_{D}$ and intentisty) for each peptide sequence. Isobaric ions are also present (e.g., at $t_{D} \sim 7.4 \mathrm{~ms}$ ); however, their low intensities do not allow an unambiguous assignment as separate peptide ions or different gas phase conformers.

Because the resulting proteolytic peptide ions are difficult to identify based solely on precursor $\mathrm{m} / \mathrm{z}$, an initial step in the IMS-MS approach is to separate and then identify undeuterated peptides ions using CID. The identification of peptide ions also serves to evaluate pepsin sequence coverage for ubiquitin. For example, ions of average $\mathrm{m} / \mathrm{z}$ 661.87 are mobility separated and isolated by the LTQ for MS/MS analysis. Figure 4.3B shows the CID fragmentation spectrum obtained upon activation of these ions. Several $b$ - and $y$-product ions are shown in the Figure 4.3B. Comparisons of these ions with insilico fragments are consistent with an assignment of the precursor ions as $[\text { FVKTLTGKTITL }+2 \mathrm{H}]^{2+}$. This sequence matches the $\mathrm{N}$-terminal end of ubiquitin, encompassing residues $\mathrm{F}^{4}-\mathrm{L}^{15}$. Crystal structure[59] comparisons show that residues $\mathrm{F}^{4}-\mathrm{T}^{7}$ form part of a beta-stranded region followed by a turn (residues $\mathrm{L}^{8}-\mathrm{K}^{11}$ ) and a second beta stranded region from $G^{10}-L^{15}$. Upon sequence identification it is worthwhile to note that even though FVKTLTGKTITL spans two separate beta-stranded regions separated by a short turn, the XIDTD is indicative of a dominat single gas-phase conformation for the +2 charge state.

\subsubsection{Matching XIDTDs Between Undeuterated and Deuterated Datasets.}


Once the ions are identified, the high reproducibility of the gas phase separation is used to match mobility profiles generated during labeling studies. Because deuterium retention does not significantly affect the mobility profile (Figure 4.7, see below), XIDTDs of deuterated peptides can be mobility matched to the respective XIDTDs of the undeuterated ions. The top trace in Figure 4.3A (red line) shows the XIDTD for ions of average $\mathrm{m} / \mathrm{z} 664.54$ generated during DHX-PD-IMS-MS of labeled ubiquitin. Figure 4.3A shows that the labeled ions of average $\mathrm{m} / \mathrm{z} 664.54$ have similar XIDTD features as ions of $\mathrm{m} / z 661.87$, with a single dominant conformation at $t_{\mathrm{D}} \sim 8.0 \mathrm{~ms}$. It is noteworthy to mention that no dataset alignment was made for the overlays presented in Figure 4.3A. Instead, a bin-by-bin comparison (based on a $200 \mu \mathrm{s} t_{D}$-bin width) is used for spectral matching. Additionally, XIDTDs offer the ability to quickly assess deuterium retention by comparing undeuterated and deuterated precursor mass spectra recorded at $t_{D} \sim 8.0 \mathrm{~ms}$. Because deuterium incorporation increases the width of the isotopic envelope, reduced peak capacity and increased spectral congestion are problematic in DHX studies.[60] By changing the delay voltage at G2 (Figure 1.1), only select peptide ions having a $t_{\mathrm{D}} \sim 8.0 \mathrm{~ms}$ are examined for improved isotopic evaluation of deuterated peptide ions. Figure 4.3C shows the isotopic distributions for unlabeled and labeled $[\text { FVKTLTGKTITL }+2 \mathrm{H}]^{2+}$ ions selected at $t_{\mathrm{D}} \sim 8.0 \mathrm{~ms}$. The top trace shows ions of $\mathrm{m} / \mathrm{z}$ 664.54 having a broader isotopic distribution that is shifted in average mass due to deuterium retention relative to unlabeled ions. In this straightforward manner, it can be observed that $[\text { FVKTLTGKTITL }+2 \mathrm{H}]^{2+}$ ion retains $\sim 5.3 \pm 0.1$ deuteriums on average. Since the sequence spans structured portions of the protein, the peptide may be expected to show a degree of deuterium retention. Although this method requires an 
initial identification analysis of unlabeled peptide ions prior to deuterium studies, we note that LC-DHX-MS/MS studies require the same process.[61] However, the relatively higher reproducibility of the IMS separation allows spectral feature matching (XIDTDs) between unlabeled and labeled samples without the need for dataset alignment.

\subsubsection{Overall Per-peptide Deuterium Analysis.}

Ubiquitin contains 144 labile hydrogens, where 72 are amide backbone, 69 are found on residue side chains and 3 sites are located on the $\mathrm{N}$ - and $\mathrm{C}$-terminus. After DHX for $\sim 30$ seconds, the resulting deuterium retention was $50 \pm 4$ deuteriums determined from the $\mathrm{m} / \mathrm{z}$ of undigested $[\mathrm{M}+6 \mathrm{H}]^{6+}$ ubiquitin ions. Figure $4.4 \mathrm{~A}$ shows secondary structural features for ubiquitin atop the primary sequence. These comprise of 5 beta-sheets (residues $\mathrm{M}^{1}-\mathrm{L}^{7}, \mathrm{G}^{10}-\mathrm{L}^{15}, \mathrm{Q}^{40}-\mathrm{F}^{45}, \mathrm{G}^{47}-\mathrm{L}^{50}$ and $\mathrm{S}^{65}-\mathrm{R}^{72}$ ), an alpha-helix $\left(I^{23}-E^{34}\right)$ and a $3 / 10$ helix $\left(L^{56}-Y^{59}\right)$. The corresponding deuterium retention for each identified peptide (MS/MS) is given in Figure 4.4C. In general, most identified peptide ions appear to originate from the terminal ends of ubiquitin. This may be due to increased pepsin accessibility to these regions despite the fast digestion time ( 22 seconds). To a certain degree, retention levels appear to map the secondary structural elements of ubiquitin. In Figure 4B, residues $M^{1}-L^{15}$, spanning two beta-sheets separated by an unstructured turn retain $7.1 \pm 0.3$ deuterium after DHX. The next peptide $\mathrm{F}^{4}-\mathrm{L}^{15}$ spans a similar portion of the $\mathrm{N}$-terminus having a calculated deuterium level of $5.3 \pm 0.1$. Here, $\mathrm{F}^{4}-\mathrm{L}^{15}$ differs by 3 residues relative to $\mathrm{M}^{1}-\mathrm{L}^{15}$ and suggests $\sim 1.8$ deuteriums are retained across $\mathrm{M}^{1}-\mathrm{F}^{4}$. Other Top-down DHX-MS/MS studies have shown that residues $M^{1}$ and $Q^{2}$ display fast intrinsic exchange rates.[62] With this in mind, these results may suggest that deuterium retention is located on $\mathrm{I}^{3}$ and $\mathrm{F}^{4}$. 
A.
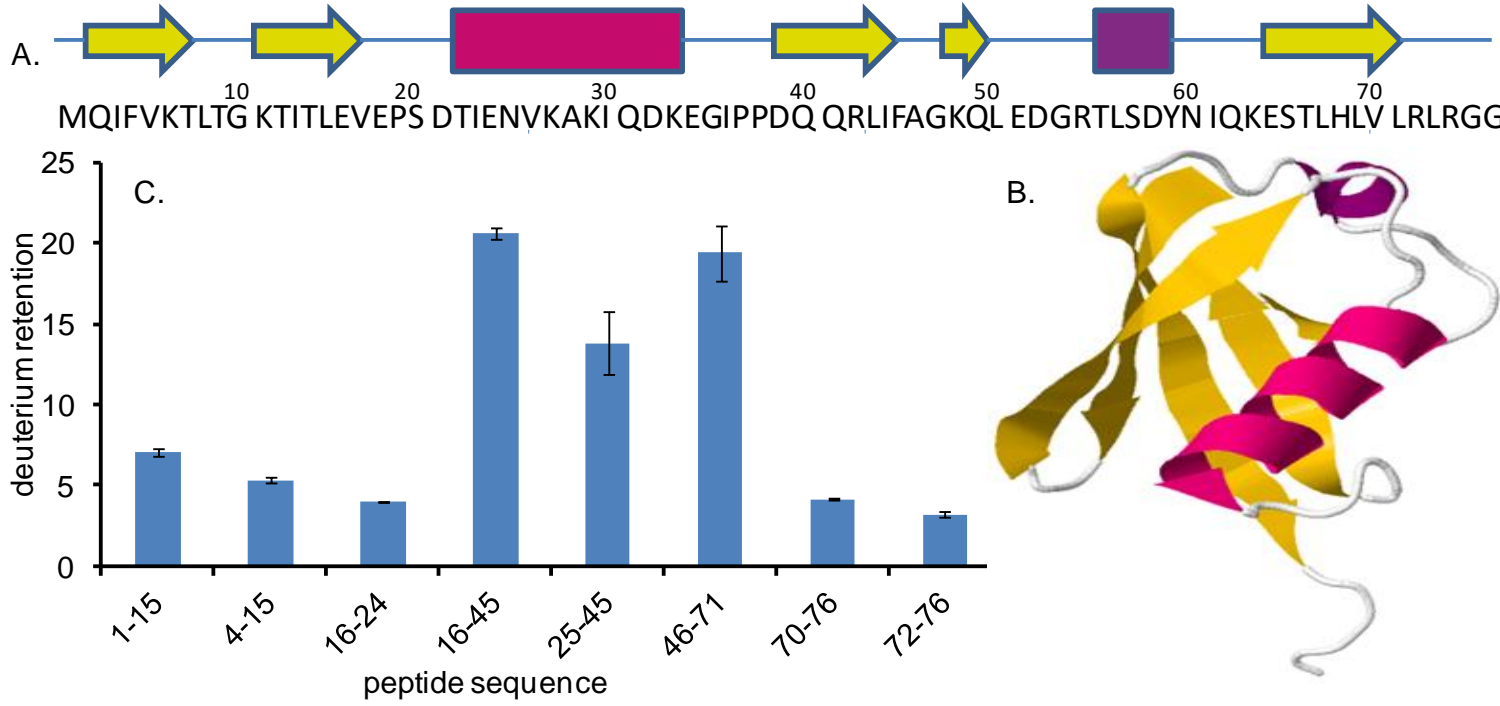

B.
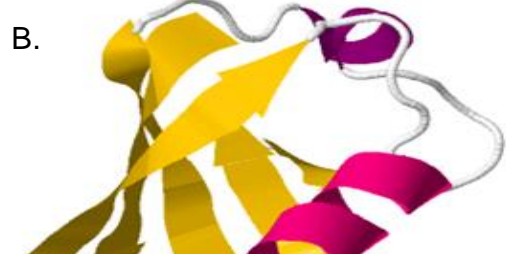

Figure 4.4. (A) Location of the secondary structural elements of ubiqutin atop the respective regions of primary sequence. Secondary structural elements are taken from (B) the ubiquitin crystal structure.[59] Areas in yellow and purple represent beta-strands and helical regions, respectively. Areas shown as blue lines are turns or unstructured regions (C) Total per-peptide deuterium content for MS/MS identified peptide ions $(\mathrm{N}=3)$.

Data for other peptide ions can be used to give a general evaluation of protection levels. For example, the peptide $E^{16}-E^{24}$ spans a portion of the second beta-strand $\left(E^{16}\right.$ $\left.E^{18}\right)$ followed by an unstructured turn $\left(E^{18}-T^{22}\right)$ and a portion of the peptide that extends into an alpha helix region $\left(I^{23}-E^{24}\right)$. Utilizing the global deuterium level, the peptide has a calculated $0.49 \pm 0.06$ deuteriums per residue. Comparatively, residues $\mathrm{N}^{25}-\mathrm{F}^{45}$ extend across the alpha-helix and a third beta-sheet containing more ( $65 \%)$ residues compared to the $\mathrm{E}^{16}-\mathrm{E}^{24}$ peptide. Here the calculated deuterium retention level perresidue is $0.69 \pm 0.09$. We note that the pre-residue retention for the former and latter peptides is highly correlated with the fraction of residues observed within regions of secondary structure. Because larger peptides often encompass several areas of secondary structural elements, deuterium location is difficult to interpret. In these cases, such peptides offer targets for MS/MS approaches using ETD. 


\subsubsection{Scrambling Control and Instrumental Studies.}

The control peptide served to elucidate instrumental parameters to mitigate scrambling by optimizing IMS source voltages and tuning the MS transfer voltages (parameters given in Appendix 1). It should be noted that the theoretical backbone amide uptake for the DIIKIIK region should include 6 deuteriums. In this study, the $[\mathrm{M}+\mathrm{H}]^{+}$ions showed a retention of $5.12 \pm 0.01$ deuteriums, where an additional backbone amide backexchanged (possibly at the backbone site $\mathrm{I}^{9}-\mathrm{K}^{10}$ ). With this assumption, the theoretical $100 \%$ and $0 \%$ scrambling line calculations were modified to include a total uptake of 5.12 and unchanged deuterium retention between $c_{8}$ and $c_{9}$ ions.

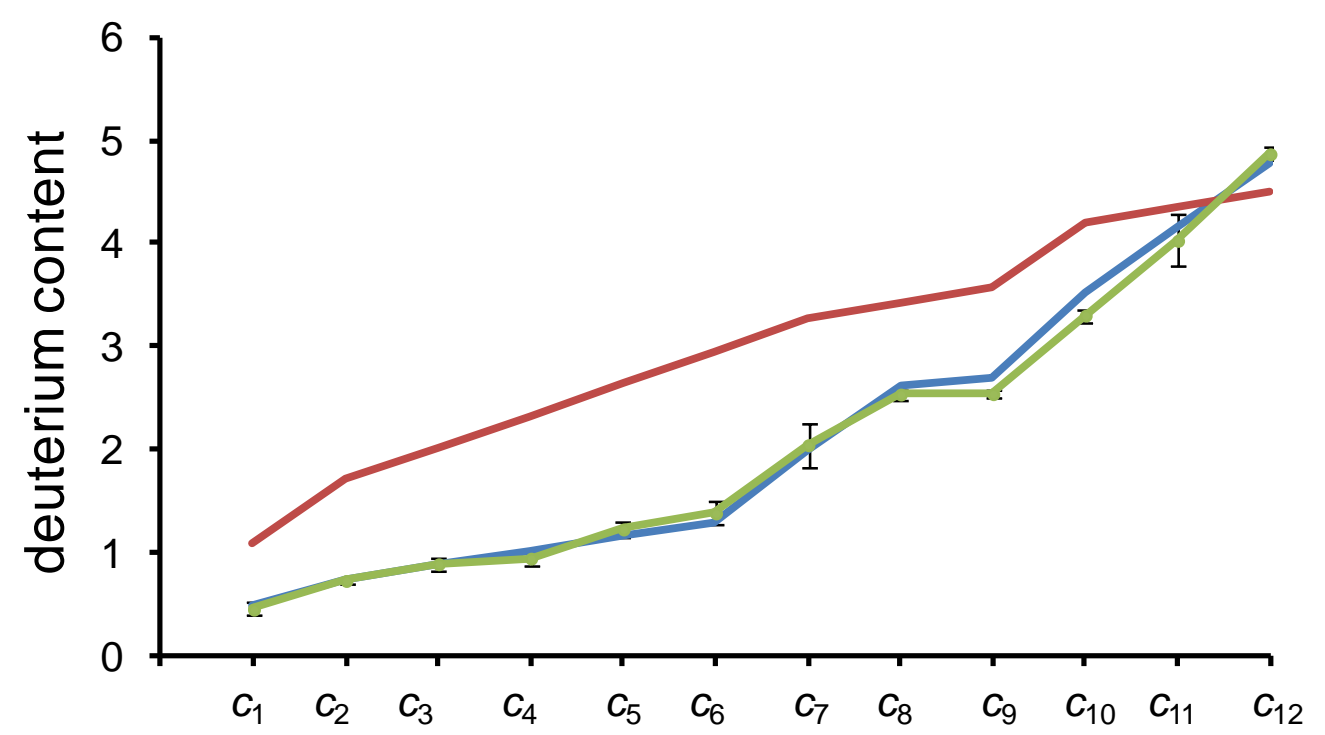

Figure 4.5. Theoretical scrambling traces showing red and blue traces for $100 \%$ (red trace) and $0 \%$ (blue trace) scrambling respectively. The green traces shows $c$-series ions generated from the DHX-IMS-ETD-MS analysis of KDDDDDIIKIIK $(n=3)$ that matches closely to the $0 \%$ scrambling model.

Upon DHX and in the absence of scrambling, the $\mathrm{N}$-terminal region should exchange nearly all deuterium for hydrogen, while the C-terminal region should retain 
deuterium at backbone amide locations. In the occurrence of scrambling, an even distribution of deuterium would be observable across all available sites. Figure 4.5 shows the theoretical retention plots generated as outlined by Zehl et al.[28] It can be seen in Figure 4.5 that the experimental data follow closely to the theoretical $0 \%$ scrambling line.

\subsubsection{IMS - ETD-MS of Deuterated Peptides for Per - Residue Analysis - Ubiquitin}

Labeled [MQIFVKTLTGKTITL+3H] $]^{3+}$ ions generated from DHX-PD-IMS-MS measurements were selected for ETD analysis. Deuterium is calculated by subtracting the average mass of a given unlabeled $c$-ion from the average mass of the respective labled $c$-ion. A lack of deuterium retention within a region of primary sequence is indicated by similar deuterium content values for adjacent $c$-ions (Figure 4.6A). Evaluation of Figure 4.6A shows deuterium retention beginning at $\mathrm{I}^{3}$ and increases to $\mathrm{T}^{7}$. However, deuterium content across residues $\mathrm{F}^{4}-\mathrm{K}^{6}$ does not change by a full deuterium. Comparison to NMR[63] and top-down MS/MS[62] studies have shown strong protection across $I^{3}-V^{5}$. As such, it is instructive to consider the online, bottom up approach with respect to tertiary interactions. Notably, the first beta strand, $M^{1}-T^{7}$, is situated between the second and fifth beta-strands collectively forming $12 \mathrm{H}$-bonding networks between $M^{1}-T^{7}$ (Figure 4.4C).[64] In the case of pepsinolysis yielding the $M^{1}$ $\mathrm{L}^{15}$ ion, this $\mathrm{H}$-bonding network is lost in the peptide and hence a degree of deuterium label may undesirably backexchange. This may be especially true at the interface of the $\mathrm{DHX}$ and quenching/digestion region where true quench conditions have not fully equilibrated. In that regard, top-down MS/MS studies appear to map the early $\mathrm{N}$ terminus of ubiquitin with better accuracy.[62, 65] 
In contrast to the $\mathrm{N}$-terminal region, other residues appear to correctly map the location of secondary structures. For example, no change in deuterium content is observed for residues $L^{8}-K^{11}$, indicating $D H X$ across this region. Here, residues $T^{7}-G^{10}$ are consistent with an unstructured turn between the first and second beta-strand (Figure 4.4A). Deuterium content (Figure 4.6A) is seen to increase across residues $\mathrm{K}^{11}$ $\mathrm{L}^{15}$, which appears to correctly correlate to the location of the second beta-strand beginning at $\mathrm{G}^{10}$. The relatively large error associated with $\mathrm{K}^{11}$ and $\mathrm{T}^{12}$ may be indicative of residue location. That is, residues that lay on the fringes of secondary structures are presumably less protected.[5] Generally, the deuterium content profile is consistent with the known crystal structure of ubiquitin (Figure 4.4A and C). Notably, these spectral consistencies further indicate that scrambling is largely mitigated, as under energizing conditions the deuterium label would be statistically randomized throughout the first few residues as well as those contained within the turn.

The online method was also used to evaluate other known structural regions of ubiquitin for comparative purposes. A prominent feature of ubiquitin is an alpha helical region across residues $\mathrm{I}^{23}-\mathrm{E}^{34}$. Using XIDTD spectral feature matching (see above), [NVKAKIQDKEGIPPDQQRLIF+4H] $]^{4+}$ ions spanning residues $\mathrm{N}^{25}-\mathrm{F}^{45}$ show a total deuterium retention of $13.8 \pm 1.9$ (Figure 4.4B) between labeled and unlabeled peptide ions. ETD analysis of deuterated [NVKAKIQDKEGIPPDQQRLIF+4H] $]^{4+}$ ions permit deuterium retention analysis across much of the alpha helical region and into the third beta-strand. Figure 4.6B shows a similar bar plot as described in Figure 4.6A for 

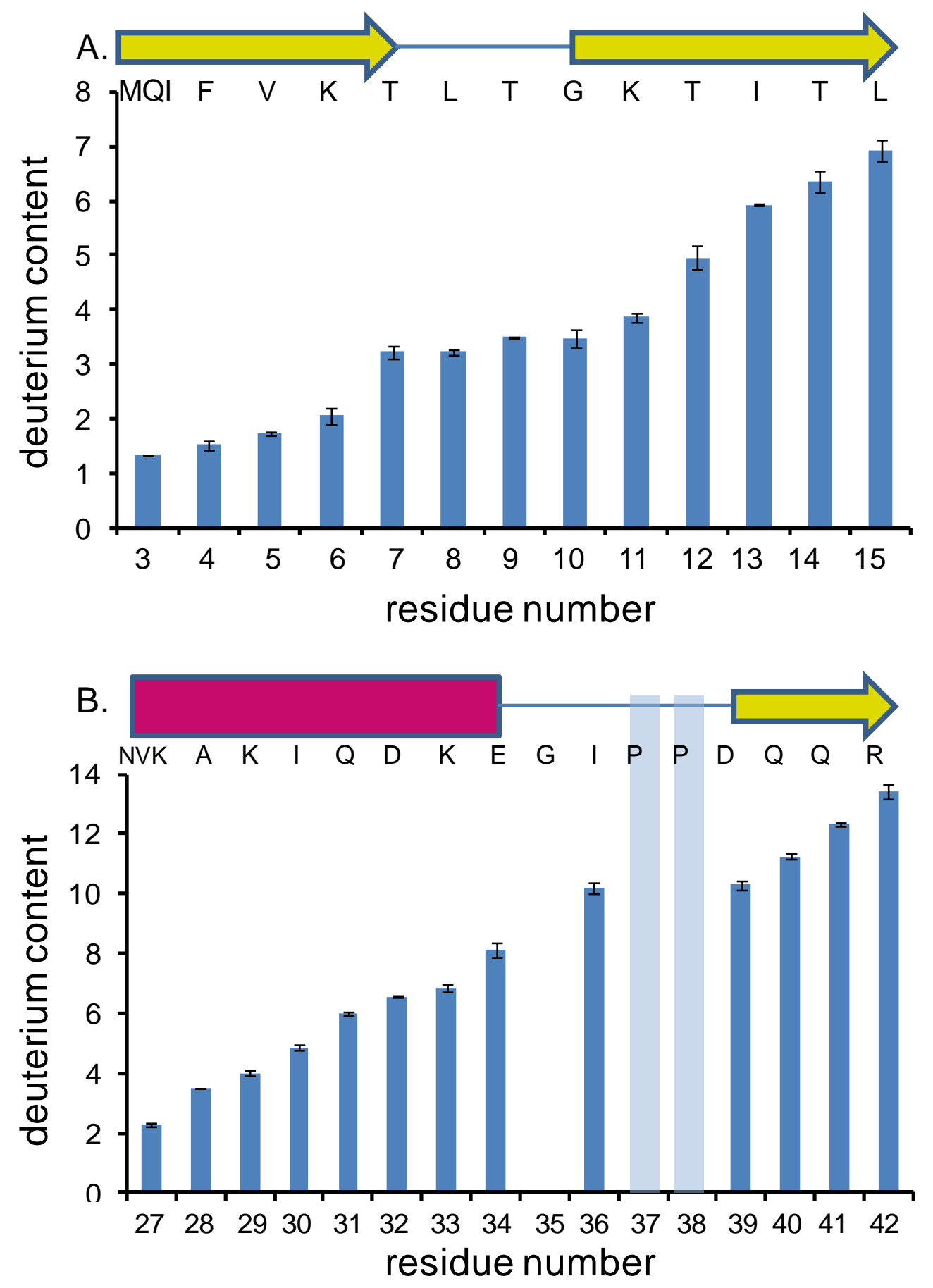

Figure 4.6. (A) Total deuterium content per residue, $n$, calculated from the $c_{n-1}$ product ions generated by ETD from labeled [MQIFVKTLTGKTITL+3H] ${ }^{3+}$ ions. The $\mathrm{N}$-terminal region of ubiquitin spanning a beta strand (residues $\left.\mathrm{M}^{1}-\mathrm{T}^{7}\right)$, turn $\left(\mathrm{L}^{8}-\mathrm{G}^{10}\right)$ and second beta strand from $\left(G^{10}-L^{15}\right)$ is also shown. (B) Total deuterium retention plot for residues $N^{25}-R^{42}$ generated by ETD of labeled [NVKAKIQDKEGIPPDQQRLIF $+4 \mathrm{H}]^{4+}$ ions. A section of the alpha-helical region and third beta strand across residues $N^{25}-E^{34}$ and $D^{39}-R^{42}$, respectively, is also shown. The error was calculated from triplicate measurements of fragment ions. 
residues $N^{25}-R^{42}$. Overall, an increase in deuterium retention is observed across $K^{27}$ $D^{32}$. Residue $K^{33}$ appears statistically similar to $D^{32}$ in deuterium content. Other studies have shown that this residue has a relatively low protection level.[62] Deuterium content is seen to increase toward $\mathrm{I}^{36}$. Interestingly, $\mathrm{I}^{36}$ is located within a turn; however $\mathrm{H}$ bonding throughout the region could permit a degree of deuterium retention.[63] The bar height of $\mathrm{I}^{36}$ is similar in height to $\mathrm{D}^{39}$ suggesting the latter residue exhibits $\mathrm{DHX}$. Residue $\mathrm{D}^{39}$ is located on the periphery of the third beta-strand and may be expected to have a reduced protection level. Residues $Q^{40}-R^{42}$ span the third beta strand, where Figure 4.6B shows a successive increase in deuterium content. As before, a higher error appears to be associated with residues that occupy the fringes of secondary structural elements. With respect to the structured regions, these data show similar trends to those studies obtained from top-down DHX-ETD-MS analysis of ubiquitin.[62, $65]$

Although the data correlate to results presented previously, they are presented here only as a qualitative comparison. The results should be treated with caution due to some limitations in the DHX experimental setup. For example, it is noted that incubation in $\mathrm{D}_{2} \mathrm{O}$ for a minimum of 40 hours will not result in complete deuteration of backbone amide sites. Indeed, experiments for compact $[\mathrm{M}+6 \mathrm{H}]^{6+}$ ions demonstrate $\sim 90 \%$ deuterium incorporation. Despite the incomplete exchange in, the results shown in Figure 4.6 demonstrate that sufficient incorporation has occurred in order to produce deuterium retention profiles portending exchange protection. Another limitation is that the deuterium content at the ESI needle tip is $\sim 16 \%$. Here we note that the flow rates of the protein sample and the exchange out solution were optimized based on ion signal 
levels on our homebuilt instrument which led to these conditions. That said, deuterium uptake simulations (Figure 4.8) demonstrate that any deuterium retention by side-chains due to the ESI solvent composition does not affect the qualitative comparison presented in this proof-of-principle study. With this in mind, improvements in HDX methods will allow more substantial structural determinations to be made in the future.

\subsubsection{Reproducibility of the On - Line DHX-PD-IMS-MS System}

The online HDX and digestion set-up was reconstructed at the start of each trial (performed on separate days) with fresh back wash and digestion solutions which allowed the assessment of the reproducibility of deuterium retention profiles, pepsin digestion and the IMS-MS measurement. Table 4.1 shows eight identified peptides that were observed within each trial corresponding to $100 \%$ protein sequence coverage. That full sequence coverage was observed in every run provides some context as to the reproducibility of the pepsin digestion for this proof-of-principle study. With regard to peptide deuterium retention between replicates $(\mathrm{N}=3)$, coefficients of variation $(\mathrm{CV})$ ranged from $0.3 \%$ to $14.0 \%$ for deuterated ions having average $\mathrm{m} / \mathrm{z}$ values of 387.8 and 613.8, respectively. These respective ions correspond to peptides from the C-terminal tail region ([VLRLRGG+2H $]^{2+}$ ions), and a portion of the alphahelical region ([NVKAKIQDKEGIPPDQQRLIF+4H] $]^{4+}$ ions).

As noted above, a particular advantage of the IMS measurement is the ability to match ions based on mobility profiles. Figure 4.7 shows the XIDTDs from eight ions observed across all replicates that were highly abundant. The most dominant spectral features observed in each XIDTD are reported as a single $t_{D}$ value in Table 4.1. Notably, the most intense feature in each XIDTD exhibits the same $t_{D}$ value in all 


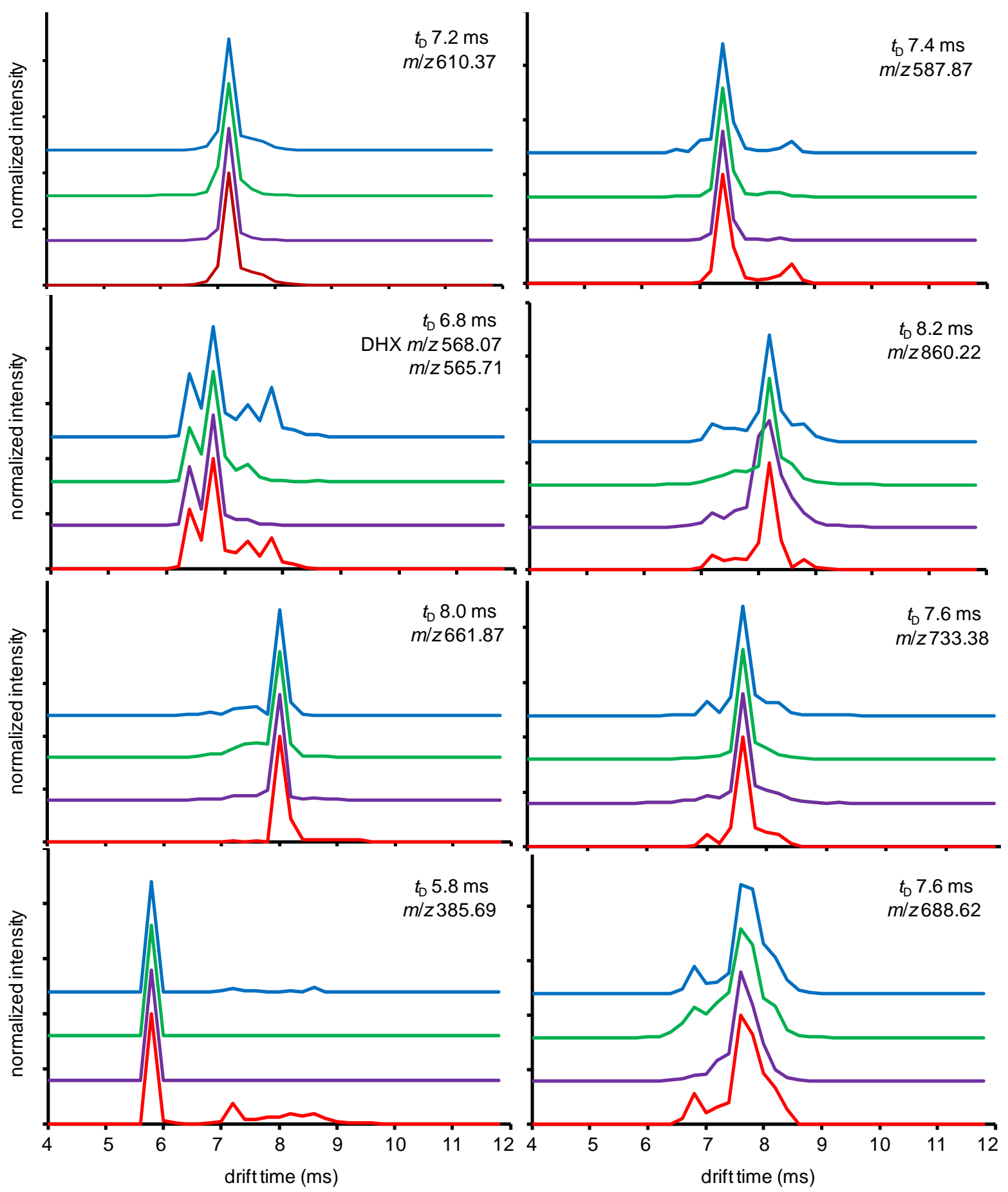

Figure 4.7. $t_{D}$ distributions for the identified peptide ions presented in Table 4.1. For each panel, the bottom trace represents the $t_{D}$ distribution obtained for the analysis of the unlabeled protein. The top three traces represent $t_{D}$ distributions collected on successive days for the labeled protein experiments. The average $\mathrm{m} / \mathrm{z}$ values of the unlabeled peptide ions and the $t_{D}$ value of the dominant dataset feature are provided in each panel. 


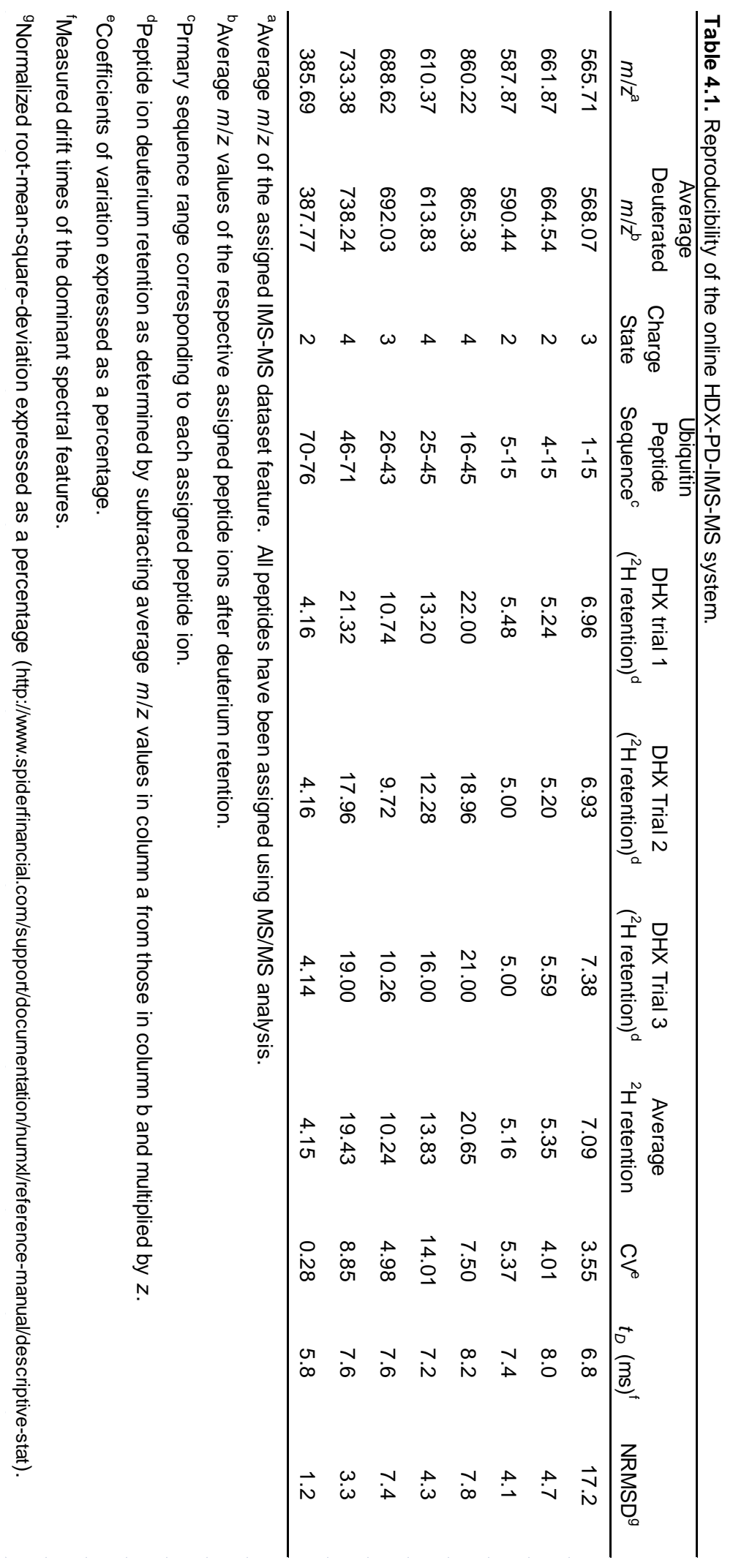


datasets including the unlabeled measurements. Although the high reproducibility of these values does not allow the determination of the error associated with the $t_{D}$ measurement, a worst case error can be estimated based on the step size of the $t_{D}$ bins. Therefore, using $\pm 0.2 \mathrm{~ms}$ as the $t_{D}$ bin size, a single bin shift would result in an estimated maximum error of $\sim 2 \%$ to $\sim 4 \%$ across the $t_{D}$ range for the observed ions. As evident from Figure 4.7, the true error is smaller and would only be determined with the use of much smaller $t_{D}$ bin sizes (significantly greater experimental times). A related concern is the reproducibility of $t_{D}$ distributions for the various ions. In order to determine the variation between the replicates with respect to XIDTDs, an average normalized root-mean-squared-deviation (NRMSD) and is described by the following equation:

$$
\frac{\sqrt{\frac{\sum\left(y_{1}-y_{2}\right)^{2}}{N_{t}}}}{y_{\max }-y_{\min }} \times 100 \%
$$

In Equation 4.1, $y$ represents the intensity at a given $t_{D}$ value for the respective (first and second) $t_{D}$ distributions and $N_{t}$ is equal to the number of $t_{D}$ values. It is noted that only $y$-values spanning the $t_{D}$ range over which features are observed are used in this calculation. Values obtained from Equation 4.1 are reported as a percentage in Table 4.1. Average NRMSD values ranged from $1.2 \%$ to $17.2 \%$ for deuterated ions having average $\mathrm{m} / \mathrm{z}$ values of 568.07 and 385.69 , respectively. In general, the average NRMSD values are quite low (<8\%) as shown in Table 4.1.This indicates high reproducibility in XIDTD profiles for separate replicate runs. It is again noted that no alignment is performed between datasets. 


\subsubsection{Assessment of the Effects of ESI Solvent Composition. A challenge of the HDX}

method employed in this demonstration is the relatively high deuterium content ( $16 \%)$ within the ESI solvent. To model the effect of ESI solvent deuterium contribution, simulations for 100 ions were performed in which the side chain HDX sites within a peptide exhibit a 0.16 probability of containing a deuterium. The increase to average $\mathrm{m} / \mathrm{z}$ for each $\mathrm{c}$ ion was then determined for the 100 ions. The deuterium contribution from the ESI solvent was then subtracted from the experimental results for the peptides shown in Figure 4.6. Figure 4.8 shows the simulated deuterium retention results for the two peptide regions described above. As evidenced by Figure 4.8, side chain deuterium incorporation from the ESI solvent would not significantly alter the overall deuterium retention profile (compared to Figure 4.6 above). That is, the relative protection as described in the manuscript is still observed. It is noted that the HDX method was largely determined by optimizing the overall peptide ion signal for this proof-of-principle study. In the future, greater effort will be utilized to optimize the HDX method in order to ensure more substantial structural determinations.

\subsubsection{Instrumental Implications and Conclusion}

This study demonstrates the utility of combining online DHX-PD and IMS-MS/MS for protein structural analysis without the need for condensed-phase separation. The high reproducibility of the linear-field, low-pressure IMS separation allows for XIDTD spectral matching of undeuterated and deuterated ions for rapid identification and deuterium retention calculations for individual peptides. For deuterium labeling studies, the IMS source, drift tube and interface regions are shown to transfer ions without excessive ion heating and deuterium scrambling. When combined with ion trapping and ETD capabilities of the LTQ, the method can reveal elements of protein secondary 


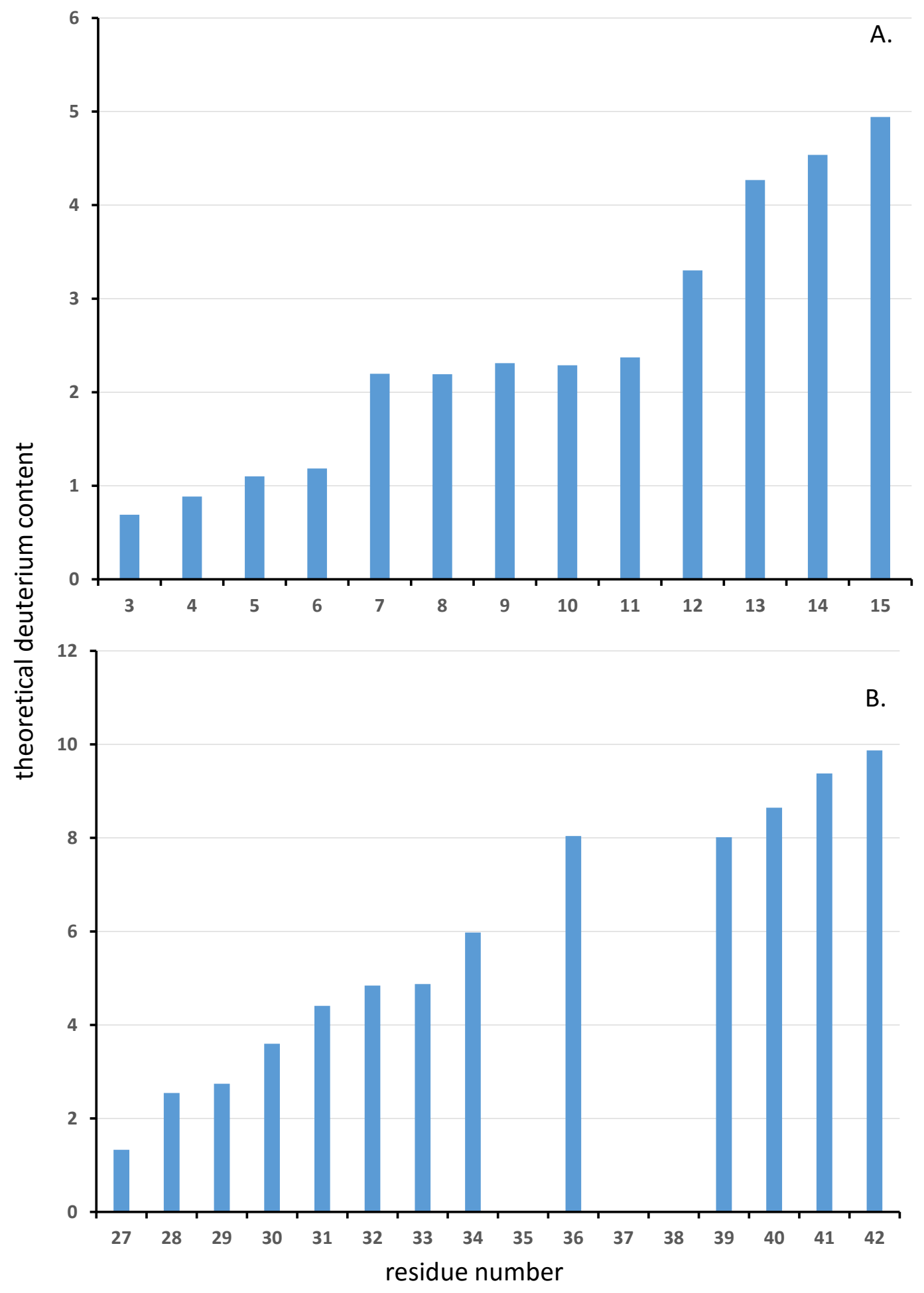

Figure 4.8. (A) Theoretical amide deuterium content per residue, $n$, calculated from the $c_{n-1}$ product ions generated by ETD from labeled [MQIFVKTLTGKTITL+3H] ${ }^{3+}$ ions. (B) Theoretical amide deuterium content plot obtained upon ETD of labeled [NVKAKIQDKEGIPPDQQRLIF $+4 \mathrm{H}]^{4+}$ ions. 
structure at a per-residue level. These data are consistent with the known secondary structural elements of ubiquitin.

Although the data provided here represent that obtained from a proof-of-principle study, it is noteworthy to mention that several improvements are envisioned to yield higher-efficiency structural determinations. Because applying non-activating transfer voltages to the IMS and MS devices in order to mitigate deuterium scrambling results in decreased sensitivity, changes in gas composition and front ion trapping funnel design[66] may improve the signal-to-noise ratio of low intensity ions without causing deuterium scrambling. Admittedly, other studies have used rapid mixing devices[67] to quickly equilibrate $\mathrm{DHX}$ and digestion regions prior to MS/MS analysis. This may further prevent the occurrence of undesirable backexchange events. Lastly, the single DHX time point is intended to serve as a rapid structural probe and does not capture protein dynamics as described by time-dependent DHX strategies. As such, the use of varying DHX times with online microfluidic devices[67-69] may yield informative kinetic studies that can be coupled to IMS-ETD-MS analysis. 


\subsection{References}

1. Engen, J.R. and D.L. Smith, Investigating protein structure and dynamics by hydrogen exchange MS. Anal Chem, 2001. 73(9): p. 256a-265a.

2. Engen, J.R., et al., Partial cooperative unfolding in proteins as observed by hydrogen exchange mass spectrometry. Int Rev Phys Chem, 2013. 32(1): p. 96-127.

3. Wei, H., et al., Hydrogen/deuterium exchange mass spectrometry for probing higher order structure of protein therapeutics: methodology and applications. Drug Discovery Today, 2014. 19(1): p. 95-102.

4. Engen, J.R., Analysis of Protein Conformation and Dynamics by Hydrogen/Deuterium Exchange MS. Analytical Chemistry, 2009. 81(19): p. 7870-7875.

5. Kaltashov, I.A., C.E. Bobst, and R.R. Abzalimov, H/D Exchange and Mass Spectrometry in the Studies of Protein Conformation and Dynamics: Is There a Need for a Top-Down Approach? Analytical Chemistry, 2009. 81(19): p. 7892-7899.

6. Kaltashov, I.A., C.E. Bobst, and R.R. Abzalimov, Mass spectrometry-based methods to study protein architecture and dynamics. Protein Science, 2013. 22(5): p. 530-544.

7. Khanal, A., et al., Pulsed hydrogen/deuterium exchange mass spectrometry for time-resolved membrane protein folding studies. J Mass Spectrom, 2012. 47(12): p. 1620-6.

8. Konermann, L., J. Pan, and Y.-H. Liu, Hydrogen exchange mass spectrometry for studying protein structure and dynamics. Chemical Society Reviews, 2011. 40(3): p. 1224-1234.

9. Shi, X., et al., Hydrogen Exchange-Mass Spectrometry Measures Stapled Peptide Conformational Dynamics and Predicts Pharmacokinetic Properties. Analytical Chemistry, 2013. 85(23): p. 1118511188.

10. Skinner, J.J., et al., Protein dynamics viewed by hydrogen exchange. Protein Sci, 2012. 21(7): p. 996-1005.

11. Keppel, T.R., B.A. Howard, and D.D. Weis, Mapping Unstructured Regions and Synergistic Folding in Intrinsically Disordered Proteins with Amide H/D Exchange Mass Spectrometry. Biochemistry, 2011. 50(40): p. 8722-8732.

12. Berkowitz, S.A., et al., Analytical tools for characterizing biopharmaceuticals and the implications for biosimilars. Nat Rev Drug Discov, 2012. 11(7): p. 527-40.

13. Mayne, L., et al., Many Overlapping Peptides for Protein Hydrogen Exchange Experiments by the Fragment Separation-Mass Spectrometry Method. Journal of The American Society for Mass Spectrometry, 2011. 22(11): p. 1898-1905.

14. Zhang, H.M., et al., Fast reversed-phase liquid chromatography to reduce back exchange and increase throughput in H/D exchange monitored by FT-ICR mass spectrometry. J Am Soc Mass Spectrom, 2009. 20(3): p. 520-4.

15. Jones, L.M., et al., Online, High-Pressure Digestion System for Protein Characterization by Hydrogen/Deuterium Exchange and Mass Spectrometry. Analytical Chemistry, 2010. 82(4): p. 1171-1174.

16. Zhang, H.-M., et al., Fast Reversed-Phase Liquid Chromatography to Reduce Back Exchange and Increase Throughput in H/D Exchange Monitored by FT-ICR Mass Spectrometry. Journal of the American Society for Mass Spectrometry, 2009. 20(3): p. 520-524.

17. Sheff, J., M. Rey, and D. Schriemer, Peptide-Column Interactions and Their Influence on Back Exchange Rates in Hydrogen/Deuterium Exchange-MS. Journal of The American Society for Mass Spectrometry, 2013. 24(7): p. 1006-1015.

18. Wu, Y., S. Kaveti, and J.R. Engen, Extensive deuterium back-exchange in certain immobilized pepsin columns used for H/D exchange mass spectrometry. Analytical Chemistry, 2006. 78(5): p. 1719-1723. 
19. Venable, J.D., W. Scuba, and A. Brock, Feature Based Retention Time Alignment for Improved HDX MS Analysis. Journal of the American Society for Mass Spectrometry, 2013. 24(4): p. 642645.

20. Rand, K., et al., ETD in a Traveling Wave Ion Guide at Tuned Z-Spray lon Source Conditions Allows for Site-Specific Hydrogen/Deuterium Exchange Measurements. Journal of The American Society for Mass Spectrometry, 2011. 22(10): p. 1784-1793.

21. lacob, R.E., J.P. Murphy, and J.R. Engen, lon mobility adds an additional dimension to mass spectrometric analysis of solution-phase hydrogen/deuterium exchange. Rapid Communications in Mass Spectrometry, 2008. 22(18): p. 2898-2904.

22. May, J.C. and J.A. McLean, Ion Mobility-Mass Spectrometry: Time-Dispersive Instrumentation. Analytical Chemistry, 2014.

23. Lermyte, F., et al., ETD Allows for Native Surface Mapping of a $150 \mathrm{kDa}$ Noncovalent Complex on a Commercial Q-TWIMS-TOF Instrument. Journal of The American Society for Mass Spectrometry, 2014. 25(3): p. 343-350.

24. Williams, J.P., et al., Identifying drug metallation sites on peptides using electron transfer dissociation (ETD), collision induced dissociation (CID) and ion mobility-mass spectrometry (IMMS). Chemical Communications, 2010. 46(30): p. 5458-5460.

25. Merenbloom, S.I., T.G. Flick, and E.R. Williams, How hot are your ions in TWAVE ion mobility spectrometry? J Am Soc Mass Spectrom, 2012. 23(3): p. 553-62.

26. Morsa, D., V.r. Gabelica, and E. De Pauw, Effective Temperature of lons in Traveling Wave Ion Mobility Spectrometry. Analytical Chemistry, 2011. 83(14): p. 5775-5782.

27. Shvartsburg, A.A. and R.D. Smith, Fundamentals of traveling wave ion mobility spectrometry. Anal Chem, 2008. 80(24): p. 9689-99.

28. Zehl, M., et al., Electron Transfer Dissociation Facilitates the Measurement of Deuterium Incorporation into Selectively Labeled Peptides with Single Residue Resolution. Journal of the American Chemical Society, 2008. 130(51): p. 17453-17459.

29. Rand, K.D., M. Zehl, and T.J. Jorgensen, Measuring the hydrogen/deuterium exchange of proteins at high spatial resolution by mass spectrometry: overcoming gas-phase hydrogen/deuterium scrambling. Acc Chem Res, 2014. 47(10): p. 3018-27.

30. Pan, J., et al., Structure and dynamics of small soluble Abeta(1-40) oligomers studied by topdown hydrogen exchange mass spectrometry. Biochemistry, 2012. 51(17): p. 3694-703.

31. Abzalimov, R.R., C.E. Bobst, and I.A. Kaltashov, A New Approach to Measuring Protein Backbone Protection with High Spatial Resolution Using H/D Exchange and Electron Capture Dissociation. Analytical Chemistry, 2013. 85(19): p. 9173-9180.

32. Lee, S., et al., Extracted fragment ion mobility distributions: A new method for complex mixture analysis. International Journal of Mass Spectrometry, 2012. 309: p. 154-160.

33. Arndt, J.R., et al., Lysine residues in the $\mathrm{N}$-terminal huntingtin amphipathic $\alpha$-helix play a key role in peptide aggregation. Journal of Mass Spectrometry, 2015. 50(1): p. 117-126.

34. Counterman, A.E., et al., Formation of peptide aggregates during ESI: Size, charge, composition, and contributions to noise. Journal of the American Society for Mass Spectrometry, 2001. 12(9): p. 1020-1035.

35. Mesleh, M.F., et al., Structural Information from Ion Mobility Measurements: Effects of the Long-Range Potential. The Journal of Physical Chemistry, 1996. 100(40): p. 16082-16086.

36. Revercomb, H.E. and E.A. Mason, THEORY OF PLASMA CHROMATOGRAPHY GASEOUS ELECTROPHORESIS - REVIEW. Analytical Chemistry, 1975. 47(7): p. 970-983.

37. Shvartsburg, A.A. and M.F. Jarrold, An exact hard-spheres scattering model for the mobilities of polyatomic ions. Chemical Physics Letters, 1996. 261(1-2): p. 86-91. 
38. Wyttenbach, T., et al., Effect of the long-range potential on ion mobility measurements. Journal of the American Society for Mass Spectrometry, 1997. 8(3): p. 275-282.

39. Blase, R.C., et al., Increased ion transmission in IMS: A high resolution, periodic-focusing DC ion guide ion mobility spectrometer. International Journal of Mass Spectrometry, 2011. 301(1-3): $p$. 166-173.

40. Clemmer, D.E., R.R. Hudgins, and M.F. Jarrold, NAKED PROTEIN CONFORMATIONS CYTOCHROME-C IN THE GAS-PHASE. Journal of the American Chemical Society, 1995. 117(40): p. 10141-10142.

41. Hoaglund, C.S., et al., Three-dimensional ion mobility TOFMS analysis of electrosprayed biomolecules. Analytical Chemistry, 1998. 70(11): p. 2236-2242.

42. Kurulugama, R.T., et al., Overtone Mobility Spectrometry: Part 1. Experimental Observations. Journal of the American Society for Mass Spectrometry, 2009. 20(5): p. 729-737.

43. Merenbloom, S.I., et al., High-resolution ion cyclotron mobility spectrometry. Anal Chem, 2009. 81(4): p. 1482-7.

44. von Helden, G., T. Wyttenbach, and M.T. Bowers, Conformation of macromolecules in the gas phase: use of matrix-assisted laser desorption methods in ion chromatography. Science, 1995. 267(5203): p. 1483-5.

45. Wittmer, D., et al., ELECTROSPRAY-IONIZATION ION MOBILITY SPECTROMETRY. Analytical Chemistry, 1994. 66(14): p. 2348-2355.

46. Bohrer, B.C., et al., Biomolecule Analysis by Ion Mobility Spectrometry, in Annual Review of Analytical Chemistry. 2008. p. 293-327.

47. Clemmer, D.E. and M.F. Jarrold, Ion mobility measurements and their applications to clusters and biomolecules. Journal of Mass Spectrometry, 1997. 32(6): p. 577-592.

48. Collins, D.C. and M.L. Lee, Developments in ion mobility spectrometry-mass spectrometry. Analytical and Bioanalytical Chemistry, 2002. 372(1): p. 66-73.

49. Kurulugama, R.T., et al., Development of a high-throughput IMS-IMS-MS approach for analyzing mixtures of biomolecules. Journal of Proteomics, 2008. 71(3): p. 318-331.

50. Shaffer, S.A., et al., A novel ion funnel for focusing ions at elevated pressure using electrospray ionization mass spectrometry. Rapid Communications in Mass Spectrometry, 1997. 11(16): p. 1813-1817.

51. Stlouis, R.H. and H.H. Hill, ION MOBILITY SPECTROMETRY IN ANALYTICAL-CHEMISTRY. Critical Reviews in Analytical Chemistry, 1990. 21(5): p. 321-355.

52. Palumbo, A.M., et al., Tandem mass spectrometry strategies for phosphoproteome analysis. Mass Spectrom Rev, 2011. 30(4): p. 600-25.

53. Palumbo, A.M., J.J. Tepe, and G.E. Reid, Mechanistic insights into the multistage gas-phase fragmentation behavior of phosphoserine- and phosphothreonine-containing peptides. J Proteome Res, 2008. 7(2): p. 771-9.

54. Ruotolo, B.T., et al., Analysis of phosphorylated peptides by ion mobility-mass spectrometry. Anal Chem, 2004. 76(22): p. 6727-33.

55. Ruotolo, B.T., et al., Distinguishing between phosphorylated and nonphosphorylated peptides with ion mobility-mass spectrometry. J Proteome Res, 2002. 1(4): p. 303-6.

56. Donohoe, G.C., et al., A New lon Mobility-Linear Ion Trap Instrument for Complex Mixture Analysis. Analytical Chemistry, 2014. 86(16): p. 8121-8128.

57. Zucker, S.M., et al., An ion mobility/ion trap/photodissociation instrument for characterization of ion structure. J Am Soc Mass Spectrom, 2011. 22(9): p. 1477-85.

58. Baker, E.S., et al., lon mobility spectrometry-mass spectrometry performance using electrodynamic ion funnels and elevated drift gas pressures. J Am Soc Mass Spectrom, 2007. 18(7): p. 1176-87. 
59. Vijay-Kumar, S., C.E. Bugg, and W.J. Cook, Structure of ubiquitin refined at 1.8 A resolution. J Mol Biol, 1987. 194(3): p. 531-44.

60. Bou-Assaf, G.M., et al., Advantages of Isotopic Depletion of Proteins for Hydrogen/Deuterium Exchange Experiments Monitored by Mass Spectrometry. Analytical Chemistry, 2010. 82(8): p. 3293-3299.

61. Konermann, L., S. Vahidi, and M.A. Sowole, Mass Spectrometry Methods for Studying Structure and Dynamics of Biological Macromolecules. Analytical Chemistry, 2013. 86(1): p. 213-232.

62. Sterling, H.J. and E.R. Williams, Real-Time Hydrogen/Deuterium Exchange Kinetics via Supercharged Electrospray lonization Tandem Mass Spectrometry. Analytical Chemistry, 2010. 82(21): p. 9050-9057.

63. Johnson, E.C., et al., Solution structure and dynamics of a designed hydrophobic core variant of ubiquitin. Structure, 1999. 7(8): p. 967-976.

64. Berman, H.M., et al., The Protein Data Bank. Nucleic Acids Research, 2000. 28(1): p. 235-242.

65. Pan, J., et al., Electron Capture Dissociation of Electrosprayed Protein Ions for Spatially Resolved Hydrogen Exchange Measurements. Journal of the American Chemical Society, 2008. 130(35): p. 11574-11575.

66. Ibrahim, Y.M., et al., Improving Ion Mobility Measurement Sensitivity by Utilizing Helium in an Ion Funnel Trap. Analytical Chemistry, 2014. 86(11): p. 5295-5299.

67. Keppel, T.R. and D.D. Weis, Analysis of Disordered Proteins Using a Simple Apparatus for Millisecond Quench-Flow H/D Exchange. Analytical Chemistry, 2013. 85(10): p. 5161-5168.

68. Rob, T. and D. Wilson, A versatile microfluidic chip for millisecond time-scale kinetic studies by electrospray mass spectrometry. Journal of the American Society for Mass Spectrometry, 2009. 20(1): p. 124-130.

69. Wilson, D.J. and L. Konermann, A capillary mixer with adjustable reaction chamber volume for millisecond time-resolved studies by electrospray mass spectrometry. Anal Chem, 2003. 75(23): p. 6408-14. 


\section{Helium Charge Transfer Dissociation Combined with Deuterium Hydrogen}

\section{Exchange Mass Spectrometry}

\subsection{Introduction: Fragmentation Techniques and Hydrogen Deuterium Scrambling}

Recent advances in tandem mass spectrometry (MS/MS) present techniques that are well suited to site-specific (per-residue) deuterium retention using both topdown[1-3] and bottom-up[4, 5] approaches. One Important aspect of pre-residue deuterium measurement is mitigating hydrogen/deuterium (HD)- scrambling. HDscrambling occurs from the collisional-heating of peptide or protein ions that result in mobile protons.[6] Because protons can be mobilized before bond dissociation, the final location of the protons is different than starting location on the precursor ion. This becomes problematic during per-residue deuterium measurements attempting to assess protein structure. It is also important to note that HD-scrambling can occur as a result of 'harsh' declustering conditions applied to the ion optics of the mass spectrometer and ion isolation due to brandband excitation.[7] Therefore special attention most also be given to and instrumental parameters to insure ion are not activate prior to fragmentation.

Electron based fragmentation processes like ECD and ETD have been used for per-residue deuterium measurements without HD-scrambling.[5, 8-11] One limitation of ECD/ETD is the difficultly in fragmenting peptides exhibiting charge states $\geq+2$. With this in mind, it would be highly desirable to have access to a fragmentation technique that could proceed via odd electron or radical-induced pathways for low charge state precursors without proton mobilization. 
Recently, a new MS/MS technique known as helium cation-charge transfer dissociation (He-CTD) of peptide and protein ions has been demonstrated using either helium cations[12] or cations from an air plasma.[13] Gaseous ions emitted during $\mathrm{He}$ CTD achieve kinetic energies sufficient to overcome the Columbic barrier with biomolecular cations. He-CTD product ions are seen to result from both vibrationallyand radically-driven dissociation pathways that resemble those formed from both CID and ECD/ETD processes.[12, 13] Of particular interest is the ability of He-CTD to produce radical fragmentation regardless of precursor ion charge state.

In the present study, the combination of DHX with He-CTD-MS is explored. Because HD-scrambling is a significant concern, a model peptide specifically designed to determine HD-scrambling[7] is used for a theoretical and experimental scrambling evaluation. These experiments are directly compared to ETD experiments, which were obtained under non-scrambling conditions. Separate experiments used an online and continuous DHX system coupled with pepsin digestion (PD) and simultaneous DHX quenching for structural elucidation of deuterated ubiquitin. Using the non-scrambling conditions found with the model peptide, DHX-He-CTD-MS results are presented in a proof-of-concept, per-residue structural evaluation of the $\mathrm{N}$-terminal region (residues 1 15) in ubiquitin. Since the N-terminal region contains both the fastest and slowest exchanging residues in the protein, an exchange-out time of $\sim 50$ seconds was sufficient to exchange unstructured areas while retaining deuterium on structured areas.

\subsection{Methods and Materials}

Ubiquitin (bovine erythrocytes, 98\%) and lyophilized pepsin (porcine, 3200-4500 units/mg protein), Deuterium oxide (99.9\%) and glacial acetic acid (99\%) were 
purchased from Sigma-Aldrich (St. Louis, MO). The model peptide (MP)

KKDDDDDIIKIIK (90.6\%) was purchased from Genscript (Piscataway, NJ, USA).

Proteins and peptides were used without further purification and all other reagents were MS grade or the equivalent.

\subsubsection{Sample Preparation}

Ubiquitin $(1.0 \mathrm{mg})$ was added to $1.0 \mathrm{~mL}$ of $\mathrm{D}_{2} \mathrm{O}(99.9 \%)$. The solution was incubated at $37^{\circ} \mathrm{C}$ for 10 days and left for more than 3 weeks at room temperature. This method allowed for $\sim 98 \%$ deuterium incorporation of ubiquitin. Pepsin solutions were prepared by adding lyophilized powder $(1.0 \mathrm{mg})$ to $1.0 \mathrm{~mL}$ acidified $18 \mathrm{M} \Omega \mathrm{H}_{2} \mathrm{O}(8 \%$ glacial acetic acid v:v) at $\mathrm{pH} \sim 2.0$.

\subsubsection{Modified Instrument}

A schematic of the online system is presented in Figure 5.1 and has been previously described.[14] Briefly, the DHX reaction followed by quenching and simultaneous digestion was performed using two micro-Tee assemblies (Upchurch Scientific Inc, Oak Harbor, Wa) connected with PEEK capillary $(1588 \mu$ m o.d. $\times 152 \mu \mathrm{m}$ i.d). Using a 500- $\mu \mathrm{L}$ syringe (Hamilton, Reno, NV, USA), a high precision syringe pumps (KD scientific Holliston, MA,USA) delivered the deuterated ubiquitin solution to the first micro-Tee assembly at a flow rate of $0.60 \mu \mathrm{L} \cdot \mathrm{min}^{-1}$. The instrument-equipped syringe pump delivered the room temperature exchange-out solution at a flow rate of 10.0 $\mu \mathrm{L} \cdot \mathrm{min}^{-1}$. DHX of deuterated ubiquitin proceeded for $\sim 47.4$ seconds over a capillary length of $50.8 \mathrm{~cm}$. A third $500-\mu \mathrm{L}$ syringe containing ice-cooled pepsin solution ( $\mathrm{pH}$ 2.0) was introduced by a KD scientific syringe pump to a second micro-Tee assembly at a flow rate of $0.80 \mu \mathrm{L} \cdot \mathrm{min}^{-1}$. $\mathrm{DHX}$ quenching and simultaneous protein digestion was 
preformed over a capillary length of $25.4 \mathrm{~cm}(\sim 30 \mathrm{sec}$ digestion time) and interfaced directly to the commercial ESI source. Resulting peptic peptides were electrosprayed into the MS instrument using a bias voltage of $4.0 \mathrm{kV}$ at a combined flow rate of 11.40 $\mu \mathrm{L} \cdot \min ^{-1}$.

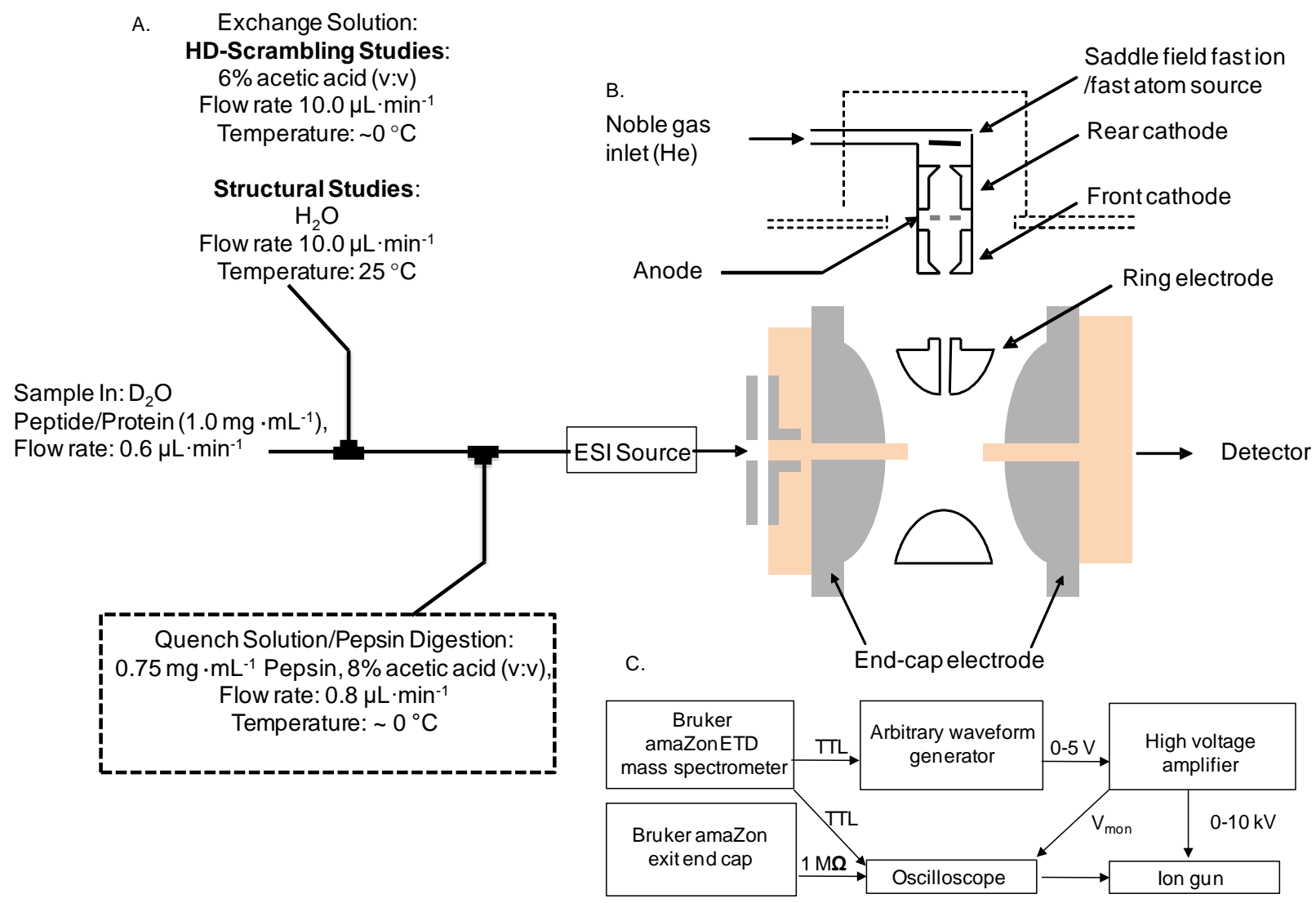

Figure 5.1. Schematic representation showing (A) the microfludic online HDX system used in HD-scrambling and structural studies. This system was directly interfaced to the commercial Bruker electrospray ionization source. The dashed-boxed region encompassing the syringe containing the pepsin solution was removed for HD-scrambling studies (B) Modified quadrupole ion trap showing the location of the saddle field ion source for the generation of $\mathrm{He}^{+}$cations. (C) The electronic components for pulsed operation during He-CTD-MS experiments.

\subsubsection{HD-Scrambling Peptide}

For the evaluation of $\mathrm{H} / \mathrm{D}$ scrambling, the model peptide KKDDDDDIIKIIK (1.0 $\mathrm{mg}$ ) was added to $1.0 \mathrm{~mL}$ of $\mathrm{D}_{2} \mathrm{O}(99.9 \%)$ and allowed to incubate for 24 hours at 
$25^{\circ} \mathrm{C}$. An online time-resolved system for continuous $\mathrm{DHX}$ was used for scrambling studies. Briefly, a 500- $\mu \mathrm{L}$ syringe (Hamilton, Reno, NV, USA) containing the deuterated peptide solution was delivered to a micro-Tee assembly using a high precision syringe pump (KD scientific Holliston, MA,USA) at a flow rate of $0.20 \mu \mathrm{L} \cdot \mathrm{min}^{-1}$. Using the instrument equipped syringe pump, acidified $18 \mathrm{M} \Omega \mathrm{H}_{2} \mathrm{O}$ (6\% acetic acid, $\left.\mathrm{pH} \sim 2.5\right)$ was introduced to the second port of micro-Tee at $10.00 \mu \mathrm{L} \cdot \mathrm{min}^{-1}$. The DHX reaction of deuterated peptide occurred over a length of $10.1 \mathrm{~cm}$ resulting in an exchange-out time of $\sim 11$ seconds. The source region was heated to $100{ }^{\circ} \mathrm{C}$ and the capillary exit potential reduced to $50 \mathrm{~V}$. A detailed list of instrumental parameters to mitigate HD-scrambling is given in the Supporting Information section. Instrumental parameters for ubiquitin deuteration studies were selected based upon the peptide exhibiting a $0 \%$ scrambling trend (see Supporting Information) during ETD experiments (see below).

\subsubsection{Mass Spectrometry Measurements and Parameters}

Full mass spectra were collected for all ions by setting the mass analyzer scan parameters over a $\mathrm{m} / \mathrm{z}$ range of 150 to 2000 and setting the ion charge control (ICC) to a target of $2 \times 10^{5}$. Precursor mass spectra were collected over 1.0 minute with 10 $\mu s c a n s / s c a n$. A full list of instrumental parameters to mitigate HD-scrambling can be found in Appendix 2.

\subsubsection{ETD Measurements}

ETD analysis was conducted on isolated precursor ions using a selection window of $\pm 10 \mathrm{Da}$ around the selected centroid $\mathrm{m} / \mathrm{z}$ value. The ICC was disabled and a trap injection time of $1.0 \mathrm{~ms}$ was used. ETD of precursor ions was enabled by the introduction of fluoranthene radicals into the QIT for $40 \mathrm{~ms}$. ETD Fragmentation spectra 
were collected for 1.0 minute in order to adequately sample the resulting isotopic distribution of product ions.

\subsubsection{He-CTD Measurements.}

He-CTD measurements were similar to ETD measurements. Briefly, precursor ions were selected using \pm 10 Da window around the centroid $\mathrm{m} / z$ value. The ICC was disabled and a quadrupole ion trap (QIT) injection time of 50 ms was used. A variable leak-valve was used to control the flow of He gas $\left(1.40 \times 10^{-5} \mathrm{mbar}\right)$ through a saddle field source (Figure 5.1). He-CTD fragmentation was performed by introducing $6 \mathrm{keV}$ helium cations into the QIT in square-wave pulse that was synchronized with the part of the scan cycle normally reserved for CID. The CID amplitude was set to zero to simply store the ions at the selected low mass cut-off value (e.g. $\mathrm{m} / \mathrm{z} 150)$ during exposure to the helium cations. For the model peptide and HD-scrambling studies, product ion spectra were collected for 2 minutes with the $\mathrm{He}^{+}$beam enabled followed by background collection for 2 minutes with the $\mathrm{He}^{+}$beam disabled. For ubiquitin studies, these respective collections periods were 3 minutes and 2 minutes. Precursor and product ion spectra were signal averaged and background subtracted prior to processing.

\subsubsection{Per-Residue Deuterium Measurements.}

Mass spectra from both ETD and He-CTD were exported as ASCII files and converted into text files (.TXT). The text files were constructed as two-column arrays of $\mathrm{m} / \mathrm{z}$ and intensity values. Using software developed in-house, deuterium retention was calculated from the deconvoluted product ion spectra by weighting $c$ - or a-ion isotopologues according to their intensity values. The software creates a text output file 
containing the average $\mathrm{m} / \mathrm{z}$ values. Average $\mathrm{m} / \mathrm{z}$ values for the unlabeled fragment ions are subtracted from those of the labeled fragment ions of the same charge state. This mass difference is reported as the amount of total deuterium retention for each detectable fragment ion.

\subsection{Results and Discussion}

\subsubsection{Peptide Control Studies and HD - Scrambling Evaluation}

To correctly evaluate the ability of He-CTD to retain a deuterium label, studies employing the model peptide (KKDDDDDIIKIIK) first used ETD experiments to determine non-activating instrumental parameters (i.e., source conditions, transfer optics potentials, and if amplitudes for ion trapping and isolation). Although the model peptide may not produce any high-order secondary structure, the peptide was designed to contain a fast exchanging $N$-terminal region and a slow exchanging $C$-terminal portion. That is, under $\mathrm{DHX}$ quench conditions, backbone amide residues including $\mathrm{D}^{7}$ through $\mathrm{I}^{12}$ retain their deuterium label for several minutes.[10] Table 5.1 shows the theoretical limits $(100 \%$ and $0 \%)$ for scrambling values calculated for the $c$-ion series of the model peptide as outlined by Zehl, et. al.[7] After online DHX-ETD-MS of $[\mathrm{M}+3 \mathrm{H}]^{3+}$ peptide ions, a comparison of experimental product ions resulting from ETD match closely to the theoretical $0 \%$ scrambling trend. This trend shows very little deuterium retention change across residues $\mathrm{K}^{1}$ through $\mathrm{D}^{6}$, followed by a sharp increase in deuteration level with each successive $c$-ion. Comparatively, under activating conditions this trend is not observed and product ions would show higher levels of deuterium content.[7] Such a case would resemble that of the $100 \%$ scrambling trend (Table 5.1).

\subsubsection{He-CTD HD-Scrambling Analysis}


Figure 5.2A shows the He-CTD spectrum for the unlabeled $[\mathrm{M}+3 \mathrm{H}]^{3+}$ peptide ions. This spectrum shows that observable $c$ ions sequence much of the model peptide. Here, it is noted that sequence coverage spans $c_{5}$ through $c_{12}$, which covers a portion of the $\mathrm{N}$-terminal region expected to not retain deuterium during $\mathrm{DHX}$ experiments. As discussed above, DHX-ETD-MS of the $[\mathrm{M}+3 \mathrm{H}]^{3+}$ ions from the deuterated peptide has been used as a control to assess parameters that mitigate deuterium scrambling before proceeding with He-CTD and structural analyses. Pertinent parameters included a capillary exit of $50 \mathrm{~V}$, Funnel 1 and 2 rf amplitudes (peak-to-peak) of $130 \mathrm{~V}$ and a trap drive of $40 \%$. A complete list of instrumental parameters can be found in Appendix 2 .

Table 5.1. HD-Scrambling analysis of $[\mathrm{KKDDDDDIIKIIK}+3 \mathrm{H}]^{3+} c$-ions generated from ETD-MS and HeCTD-MS

\begin{tabular}{|c|c|c|c|c|c|c|c|}
\hline Residue $^{a}$ & $\begin{array}{l}\text { Residue } \\
\text { number }\end{array}$ & $\begin{array}{c}\text { Theoretical } 100 \% \\
\text { Scrambling }^{\mathrm{c}}\end{array}$ & $\begin{array}{c}\text { Theoretical } 0 \% \\
\text { scrambling }^{\mathrm{c}}\end{array}$ & $\begin{array}{c}\text { ETD } \\
\text { Experimental }^{d}\end{array}$ & ETD CV ${ }^{\mathrm{e}}$ & $\begin{array}{l}\text { Experimental } \\
\quad(c \text {-ion })^{d}\end{array}$ & $\begin{array}{l}\text { CTD CV } \\
(c \text {-ion })^{\mathrm{e}}\end{array}$ \\
\hline$\overline{\mathrm{K}}$ & 1 & na & na & nd & "na & " na & na \\
\hline $\mathrm{K}$ & 2 & 1.32 & 0.61 & nd & na & nd & na \\
\hline $\mathrm{D}$ & 3 & 2.07 & 0.96 & 0.92 & 5.6 & nd & na \\
\hline $\mathrm{D}$ & 4 & 2.44 & 1.14 & 1.22 & 5.3 & nd & na \\
\hline $\mathrm{D}$ & 5 & 2.82 & 1.31 & 1.33 & 3.2 & nd & na \\
\hline $\mathrm{D}$ & 6 & 3.19 & 1.49 & 1.51 & 4.8 & 1.48 & 6.4 \\
\hline $\mathrm{D}$ & 7 & 3.57 & 1.66 & 1.58 & 6.1 & 1.81 & 20.2 \\
\hline I & 8 & 3.95 & 2.39 & 2.46 & 3.3 & 2.78 & 4.5 \\
\hline i & 9 & 4.13 & 3.03 & 3.18 & 4.1 & 3.12 & 4.9 \\
\hline $\mathrm{K}$ & 10 & 4.32 & 3.67 & 3.57 & 2.7 & 3.75 & 2.9 \\
\hline I & 11 & 5.07 & 4.57 & 4.58 & 3.2 & 4.82 & 3.2 \\
\hline I & 12 & 5.26 & 5.21 & 5.57 & 1.5 & 5.51 & 1.2 \\
\hline $\mathrm{K}$ & 13 & 5.45 & 6.00 & 6.22 & 0.4 & 5.97 & 0.7 \\
\hline
\end{tabular}

a. Primary Sequence from model peptide (KKDDDDDIIKIIK).

b. Assigned amide backbone number from the model peptide (KKDDDDDIIKIIK). Note the first amide heteroatom begins at residue 2 .

c. Theoretical deuterium content values for $c$-ions generated from ETD-MS of [KKDDDDDIIKIIK $+3 \mathrm{H}]^{3+}$ precursor ions.

d. Average experimental deuterium content obtained by subtracting the average $\mathrm{m} / \mathrm{z}$ of the labeled fragment ion from that of the unlabeled fragment ion.

e. Coefficient of variation expressed as a percentage.

f. Average experimental deuterium content obtained by subtracting the average $\mathrm{m} / \mathrm{z}$ of the labeled fragment ion from that of the unlabeled fragment ion. 

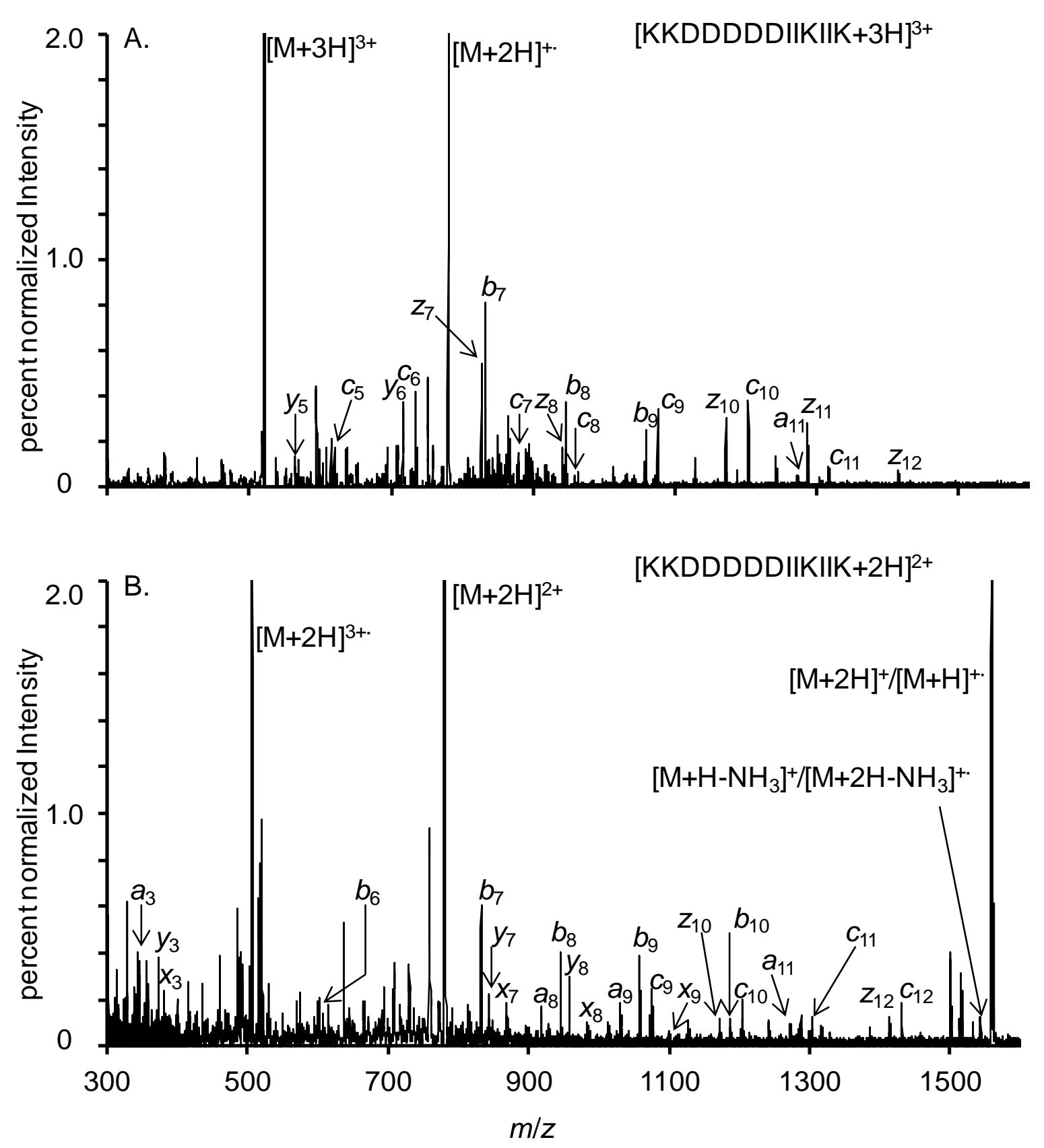

Figure 5.2. (A) He-CTD-MS spectrum of [KKDDDDDIIKIIK $+3 \mathrm{H}]^{3+}$ precursor ions. Several product ions resulting from various fragmentation pathways are labeled. (B) MS-CTD spectrum of $[K K D D D D D I I K I I K+2 H]^{2+}$ precursor ions. Identified product ions resulting from CTD are labeled. Both Figure panels $A$ and $B$ have been normalized to respective precursor ion intensities and displayed as a percentage. 
produced Table 5.1 gives per-residue deuterium retention for the deuterated model peptide observed upon DHX-He-CTD-MS of the $[\mathrm{M}+3 \mathrm{H}]^{3+}$ peptide ions. Identified $c$ and a ions resulting from He-CTD of the precursor ions were selected for direct comparisons to the theoretical HD-scrambling values (Table 5.1). Here (Table 5.1), product ions generated by He-CTD appear to match the $0 \%$ scrambling values established during the ETD control analysis. In general, coefficients of variation are less than $20 \%$ for replicate $(\mathrm{N}=3)$ He-CTD trials. Similar to ETD studies, He-CTD product ions (Table 5.1) show that the deuteration content assessment begins at a relatively low level ( $c_{5}$ and $a_{3}$ ions).

\subsubsection{He-CTD Mechanistic Insights for c - ions}

Because of the short interaction times, He-CTD is presumed to follow vertical activation (not adiabatic), and has been shown to fragment neutral molecules with appearance potentials on the order of $30 \mathrm{eV}$.[12] He-CTD therefore activates precursor ions through electronic and vibration modes. Fragment ions that result from both vibrational and radical (electronic) processes. Fragment ions can be seen in Figure 5.2A and 5.2B where several $y, b, c$ and $z$ ions were identified. However, it is instructive to consider the $c$ ion series from our tandem MS measurements. He-CTD is performed with $\mathrm{He}^{+}$cations emitted with high kinetic energy $(6 \mathrm{KeV})$ from the saddle field source. The main purpose of kiloectronvolt energies is to overcome the Coulombic barrier of cation/cation reactions. Perhaps because of inelastic transfer of energy during result from proximal reagent $\mathrm{He}^{+}$ions abstracting an electron (EA $\left.\sim 24.6 \mathrm{eV}\right)$ and creating a hole in the precursor ions. In turn, the electron deficient ion undergoes radical fragmentation, where the abstraction of an electron at the carbonyl leads to a McLafferty 
rearrangement of an amide that is radical-driven. $\mathrm{A} \mathrm{y}-\mathrm{H}^{\prime}$ transfer from the side chain to the carbonyl produces a hydroxyl group and cleavage of the N-Ca bond.

In contrast to $c$ ions produced by ECD/ETD, this ion carries a positive charge at the hydroxyl group that is electron deficient. However, deconvolution of the isotopic distributions for He-CTD-generated $c$ ion distributions that are very similar to those generated from ETD (Figure 5.3). That is, when considering singly-charged $c$ ions from ETD, the charge site is presumably a protonated basic residue and would be $1 \mathrm{Da}$ heavier than an oxidized, singly-charge $c$ ion from He-CTD. It is possible that the $c$ ions from He-CTD involve a double-hydrogen transfer during the rearrangement process. It is noteworthy to mention that several highly charged fragment ions may be observed in the He-CTD spectrum (Figure 5.2A and B) and are not clearly distinguishable for identification. The formation of $c$ - and $z$-ions via electron transfer processes formed by irradiation of excited species $[15,16]$ has been previously reported.charge transfer, the activation energy obtained through $\mathrm{He}-\mathrm{CTD}$ exceeds the electron affinity of the $\mathrm{He}^{+}$ cation and is on the order of $30 \mathrm{eV}$.[12] The formation of $c$ ions can

Much like other electron based techniques, He-CTD fragmentation appears to occur rapidly and reflects a high energy, radical-driven mechanism. That is, dissociation occurs without significant proton mobilization or HD-scrambling. Furthermore, the modified QIT is operated at a relatively higher pressure (due to the saddle source and He gas flow) whereby any vibrationally excited ions are rapidly thermalized. With this in mind, the experimental results demonstrate that $c$ ions resulting from He-CTD retain solution deuterium uptake levels. 
In general, ETD and He-CTD product ions are similar. For example, Figure 5.3 shows a comparison between several $c$-ions as well as the charge reduced, singlycharged ions generated from both fragmentation methods. Both fragmentation techniques are very similar with regard to deuterium retention, isotopic distribution and relative intensity between isotopologues. This high degree of similarity further indicates, at least for these distinguishable ions, that He-CTD fragmentation of a 3+ precursor can proceed without HD-scrambling.
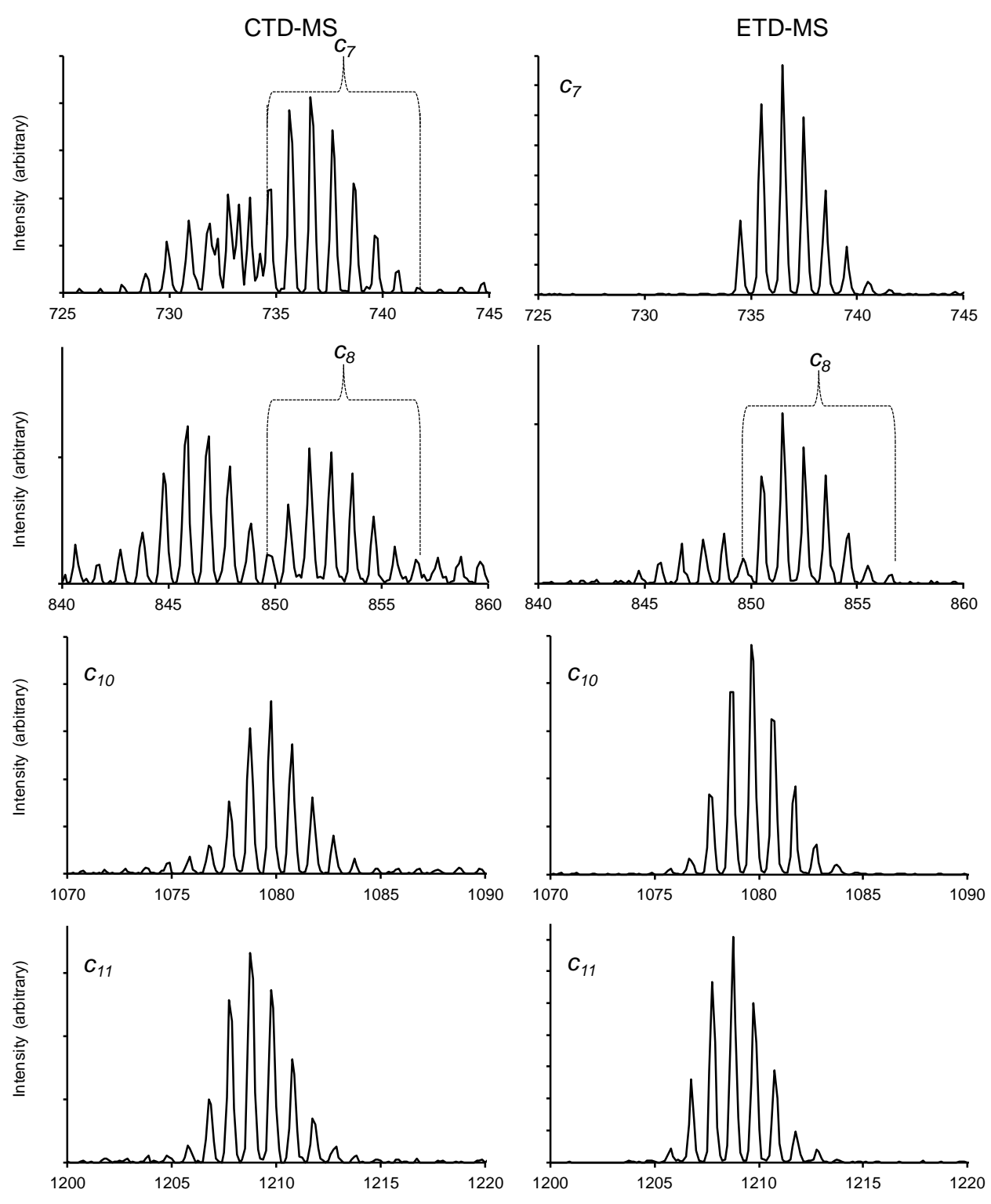

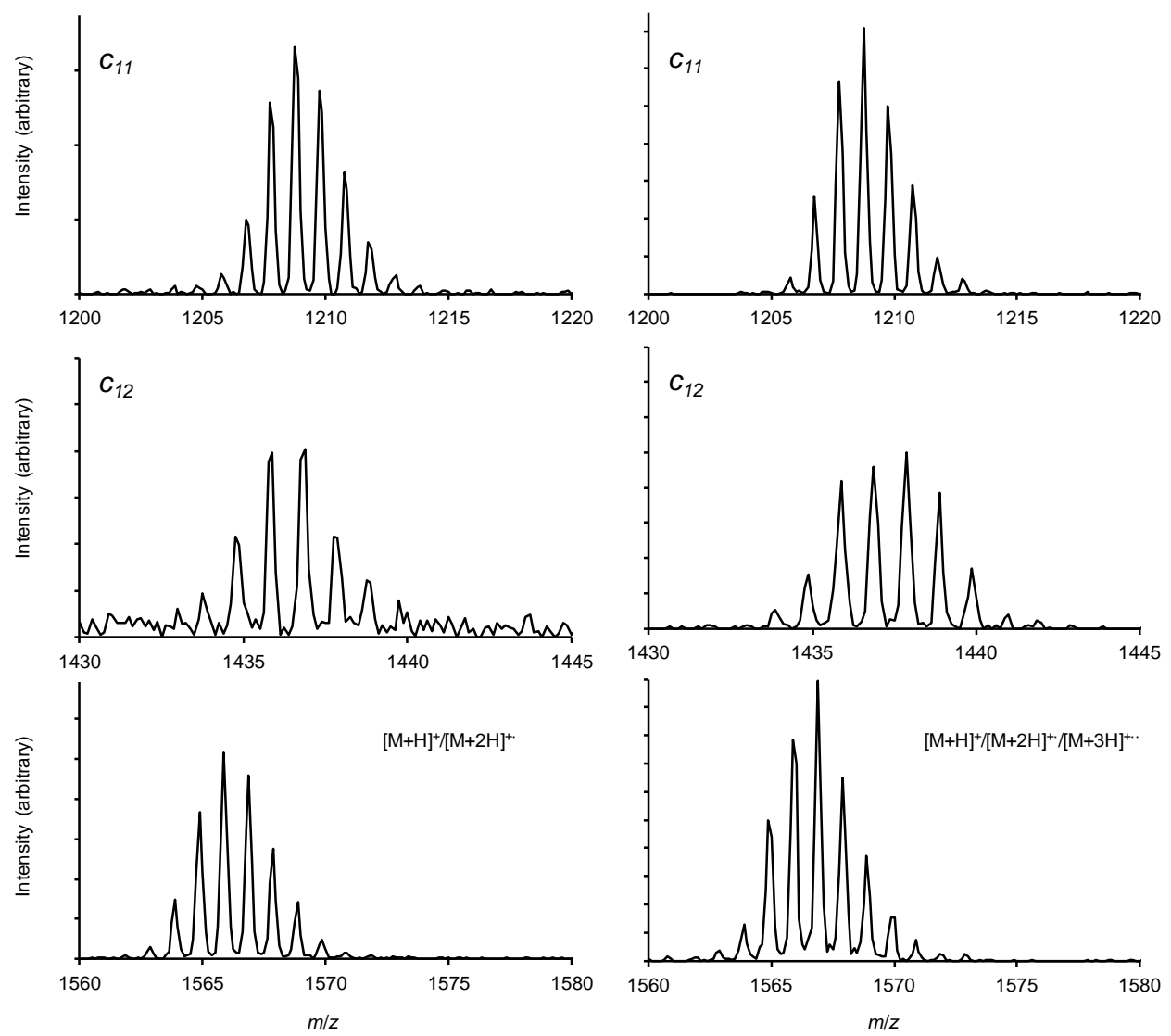

Figure 5.3. Left and right panels show identified deuterated $c$-ions resulting from HeCTD-MS and ETD-MS of KKDDDDDIIKIIK after on-line DHX respectively. These panels allow comparisons to be made based on the deuterated isotopic distribution and relative abundances of the isotopolgoues. For the respective panels, these ions were used to generate values in Table 5.1. All spectra were collected under non-activating instrumental parameters. Each panel has resulted from a single replicate from the respective fragmentation techniques.

\subsubsection{Ammonia Neutral Loss and N-terminal Scrambling}

Due to the complexity of He-CTD fragmentation spectra, the wider isotopic distribution of deuterated product ions and the relatively low resolution of the QIT, some ions are not well resolved and accurate deuterium content determinations were not possible. The compilation of these limitations has resulted in reduced sequence coverage during DHX-He-CTD studies relative to ETD. This is especially true for the $\mathrm{N}-$ 
terminal region of the model peptide, which is a region that is useful for assessing scrambling. Although the larger He-CTD $c$ ions match the $0 \%$ scrambling values (Table 5.1), it should be noted that some studies have indicated a uniform deuterium content increases across these peptides.[11] In part, this is due to the higher population of heteroatom sites that become populated under energizing processes. With this in mind, other studies have shown that ammonia neutral loss of the $\mathrm{N}$-terminal region following ETD can be used to assess scrambling in peptides.[10] Evaluation of the He-CTD spectrum for $[\mathrm{M}+2 \mathrm{H}]^{2+}$ ions form the model peptide (Figure 5.2B) shows a notable abundance of intact precursor ions formed via electron transfer or $\mathrm{H} \cdot$ transfer resulting in charge reduced $[\mathrm{M}+\mathrm{H}]^{+} /[\mathrm{M}+2 \mathrm{H}]^{+\cdot}$ molecular ions. Similar observations have been reported for $[\mathrm{M}+2 \mathrm{H}]^{2+}$ angiotensin ions irradiated with a beam of high-energy plasma cations (air), in which electron transfer appeared to be the predominant mechanism for charge reduction.[13] ETD did not produce adequate fragmentation for discussion. Evaluation of the isotopic distribution (Figure 5.2B) of the charge-reduced molecular ion shows that it has a calculated mass that is $\sim 1.4$ Da greater than the average mass of the peptide. Also present in the He-CTD spectrum of $[\mathrm{M}+2 \mathrm{H}]^{2+}$ ions (Figure $5.2 \mathrm{~B}$ ) is the presence of ammonia-loss product ions $\left(\left[\mathrm{M}+\mathrm{H}-\mathrm{NH}_{3}\right]^{+} /\left[\mathrm{M}+2 \mathrm{H}-\mathrm{NH}_{3}\right]^{+}\right)$. Figure $5.4 \mathrm{~A}$ and $\mathrm{B}$ show the these ions and the charge reduced ions after He-CTD of unlabeled $[\mathrm{M}+2 \mathrm{H}]^{2+}$ percursor ions, respectively. The difference between ions is calculated at $16.6 \pm 0.1 \mathrm{Da}$ and is consistent the loss of ammonia for $\left[\mathrm{M}+\mathrm{H}-\mathrm{NH}_{3}\right]^{+} /\left[\mathrm{M}+2 \mathrm{H}-\mathrm{NH}_{3}\right]^{+\cdot}$ ions. The comparison of the charge-reduced ion and ammonia neutral loss ions can be used to monitor scrambling as performed previously for ETD.[10] 
16.6 Da

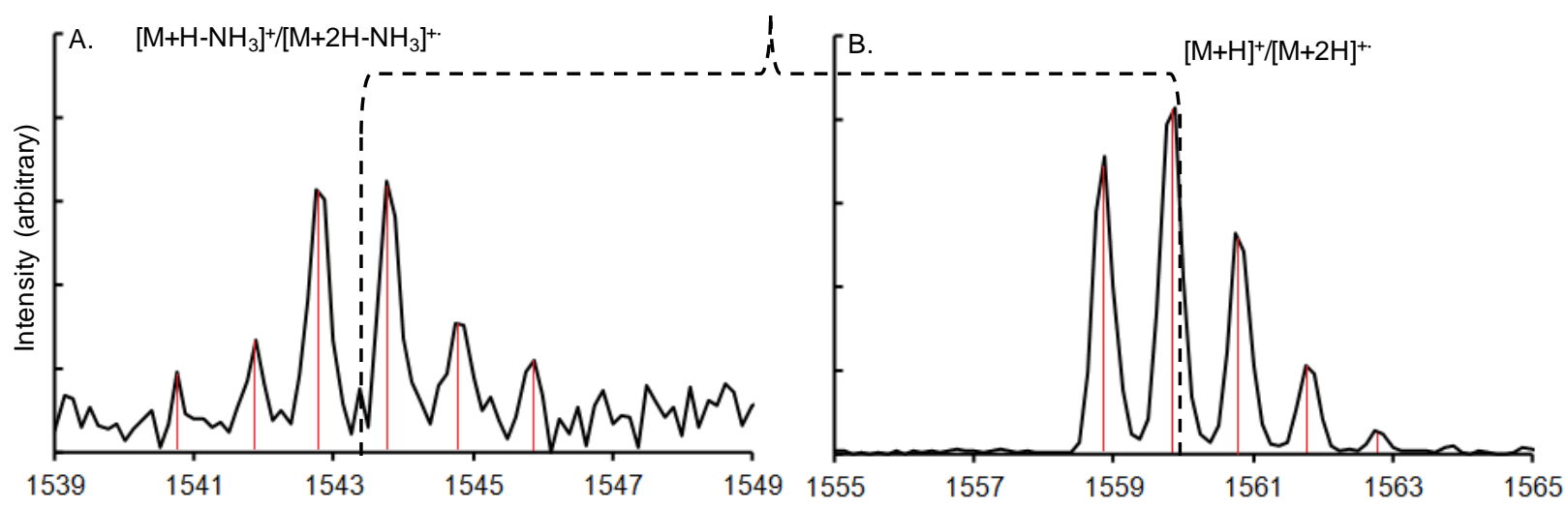

16.6 $\mathrm{Da}$

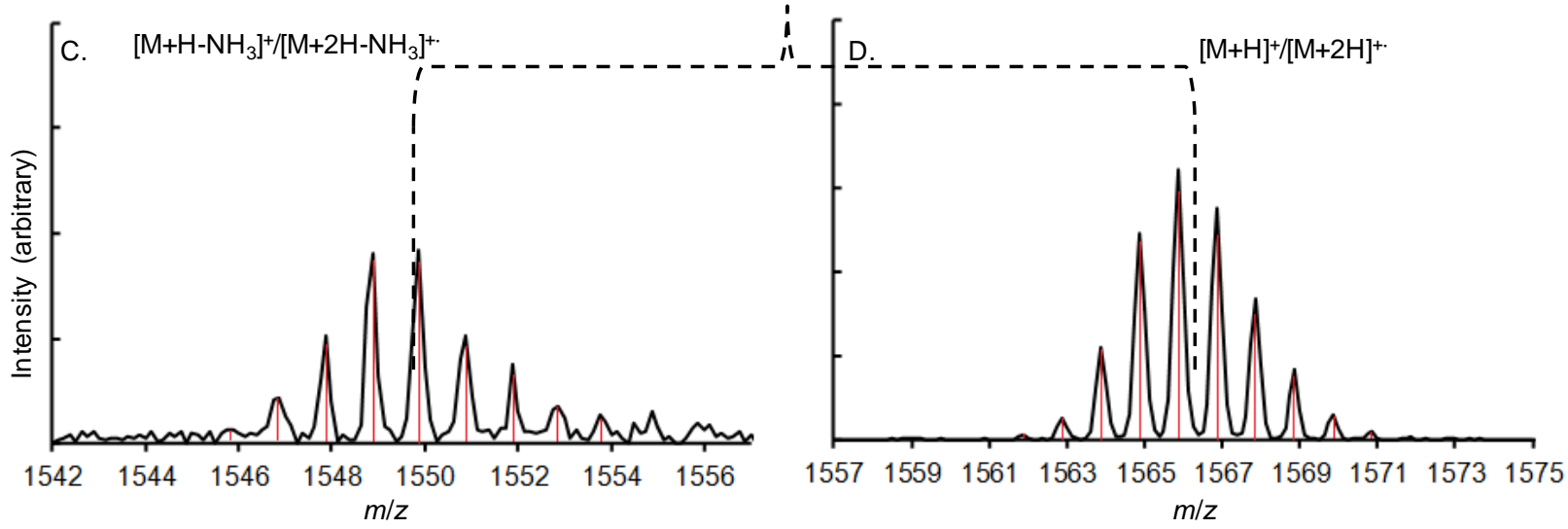

Figure 5.4. Isotopic distributions for (A) $\left[\mathrm{M}+\mathrm{H}-\mathrm{NH}_{3}\right]^{+} /\left[\mathrm{M}+2 \mathrm{H}-\mathrm{NH}_{3}\right]^{+\cdot}$ ions and (B) $[\mathrm{M}+\mathrm{H}]^{+} /[\mathrm{M}+2 \mathrm{H}]^{+}$ions originating after MS-CTD of unlabeled $[\mathrm{M}+2 \mathrm{H}]^{2+}$ model peptide precursor ions. Isotopic distributions for (C) $\left[\mathrm{M}+\mathrm{H}-\mathrm{NH}_{3}\right]^{+} /\left[\mathrm{M}+2 \mathrm{H}-\mathrm{NH}_{3}\right]^{+\cdot}$ ions and (D) $[\mathrm{M}+\mathrm{H}]^{+} /[\mathrm{M}+2 \mathrm{H}]^{+}$ions generated from HDX-MS-CTD of $[\mathrm{M}+2 \mathrm{H}]^{2+}$ model peptide precursor ions. The red lines show the centroid for each isotopologue used for $\mathrm{m} / \mathrm{z}$ averaging calculations. Black dashed lines represent the average $\mathrm{m} / \mathrm{z}$ determined from the isotopologues. The difference in the average $\mathrm{m} / \mathrm{z}$ values between adjacent panels (i.e. A-B, C-D) were used for determining HD-scrambling (see text for details). In each adjacent panel the mass difference was calculated as the loss of ammonia.

He-CTD studies for the $[\mathrm{M}+3 \mathrm{H}]^{3+}$ or $[\mathrm{M}+2 \mathrm{H}]^{2+}$ percursor ions did not result in a full series of $c$ ions required to fully evaluate HD-scrambling. Figures $5.4 \mathrm{C}$ and $5.4 \mathrm{D}$ shows the ammonia-loss and charge reduced ions upon DHX-He-CTD of $[\mathrm{M}+2 \mathrm{H}]^{2+}$ precursor ions, respectively. The $\mathrm{m} / \mathrm{z}$ difference $(16.6 \pm 0.1 \mathrm{Da})$ is very similar to that determined for the corresponding unlabeled ions. A statistical model[10] that considers 
the overall deuterium content level and all exchangeable sites suggest that, in the case of $100 \%$ HD-scrambling, the deuterium content of the ammonia-loss ions would theoretically retain 5.7 deuteriums. Notably, the deuterium retention between the labeled and unlabeled ammonia-loss ions revealed a deuterium retention value of $6.3 \pm$ 0.1 . The same total deuterium retention is also observed for the respective charge reduced ions (Figures 5.4B and 5.4D).

The agreement between the respective labeled and unlabeled product ions indicates that scrambling is not observed during the He-CTD fragmentation processes. That is, upon neutral ammonia loss from a precursor ion, the total deuterium level would be less than that of the intact ion. These results further indicate that HD-scrambling during the He-CTD process is largely not observed for the model peptide which has been specifically designed for HD-scrambling studies.[7]

\subsection{3. $D H X-H e-C T D-M S$ Structural Determinations}

With complementary scrambling models indicating that He-CTD generates $c$ ions without proton mobilization, a proof-of-concept study was used to demonstrate structural determinations using ubiquitin as the model protein. Ubiquitin contains 144 labile hydrogens, where 72 are amide backbone, 69 are found on residue side chains and 3 sites are located on the $\mathrm{N}$ - and $\mathrm{C}$-terminus. Using a continuous online microfluidic system, as described previously, DHX of labeled ubiquitin proceeded for $\sim 48$ seconds and resulted in the retention of $\sim 46 \pm 1$ deuteriums. This value was determined from the average $\mathrm{m} / \mathrm{z}$ of undigested $[\mathrm{M}+6 \mathrm{H}]^{6+},[\mathrm{M}+7 \mathrm{H}]^{6+}$ and $[\mathrm{M}+8 \mathrm{H}]^{6+}$ ubiquitin ions. Presumably these deuteriums are located in structured regions of the protein rendering 


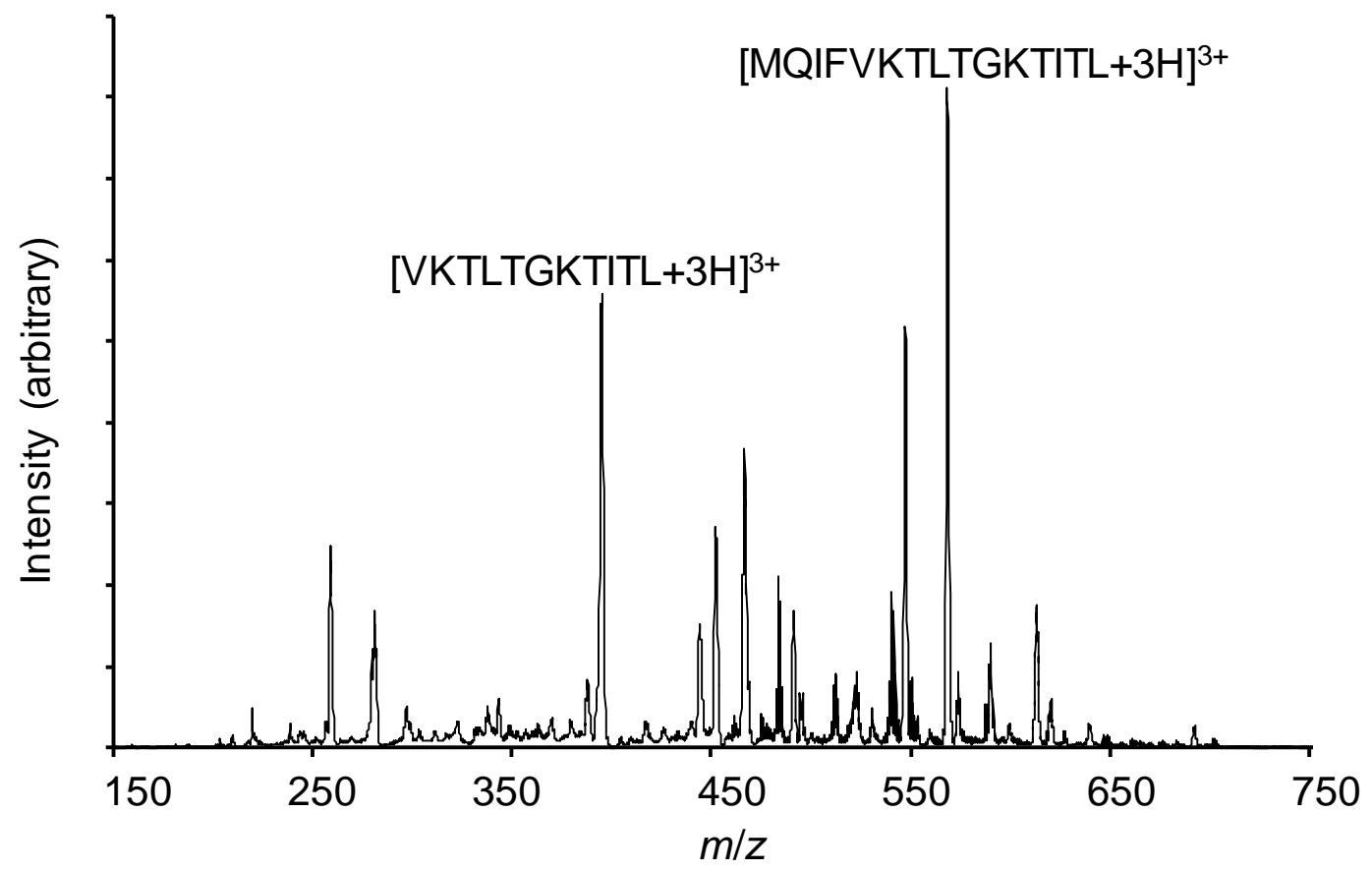

Figure 5.5. Full mass spectrum resulting from a single analysis of DHX-PD-MS of deuterated ubiquitin. The precursor ions used for He-CTD-MS and ETD-MS are labeled in the spectrum

them inaccessible to exchange during the time frame of the DHX process. For reference, Figure 4.4 (Chapter 4) shows the secondary structural features for ubiquitin as a function of the primary sequence. Known structural regions are composed of 5 beta-sheets (residues $M^{1}-L^{7}, G^{10}-L^{15}, Q^{40}-F^{45}, G^{47}-L^{50}$ and $\left.S^{65}-R^{72}\right)$, an alpha-helix $\left(I^{23}\right.$ $\left.E^{34}\right)$ and a $3 / 10$ helix $\left(L^{56}-Y^{59}\right)$.

Figure 5.5 shows the spectrum for a single replicate of labeled ubiquitin that has undergone the $\mathrm{DHX}$ and pepsin digestion (PD) prior to MS analysis. In general, most identified peptide ions appear to originate from the terminal ends of ubiquitin. These observations are similar to previous analyses using online DHX-PD-MS. This system was shown to be highly reproducible with respect to the observed peptide ions, the peptide deuterium content and the relative ion abundances.[14] Here, it is noted that the 
goal of this work is to perform He-CTD on peptides originating from structured regions of labeled ubquitin using an online DHX-PD-MS microfludic system. The analysis is therefore limited to two peptide ions of the highest abundance that were present in all replicate studies.

\subsubsection{Per-Residue ETD - MS Analysis: Control}

[MQIFVKTLTGKTITL+3H] ${ }^{3+}$ ions generated from DHX-PD-MS measurements were selected for ETD analysis having a total deuterium retention level of $8.7 \pm 0.6$. As before, deuterium content per-residue is calculated by subtracting the average mass of a given labeled $c$-ion from the average mass of the respective unlabeled $c$-ion. A lack of deuterium retention within a region of primary sequence is indicated by similar deuterium content levels for adjacent $c$ ions. Evaluation of Figure 5.6A (red bars) shows deuterium retention beginning at $\mathrm{I}^{3}$ and increasing to $\mathrm{T}^{7}$. NMR $[17,18]$ and top-down MS/MS[3, 19] studies have shown strong protection across this region. In general, deuterium residue levels appear to correctly map the location of secondary structural elements. For example, no change in deuterium content is observed for residues $L^{8}-K^{11}$ and values for residues $T^{7}-G^{10}$ are consistent with an unstructured turn between the first and second beta-strands. The deuterium content level (Figure 5.6A) is observed to increase across residues $\mathrm{K}^{11}-\mathrm{L}^{15}$, which correctly correlates with the location of the second beta-strand (beginning at $\mathrm{G}^{10}$ ). Evaluating the deuterim content levels between residues $\mathrm{G}^{10}$ and $\mathrm{K}^{11}$ shows a change by $\sim 0.43$ deuteriums. This small change suggests that residues on the fringe of secondary structural elements are less protected than residues that occupy locations within these elements.[20] For the ETD analysis of $[\mathrm{MQIFVKTLTGKTITL}+3 \mathrm{H}]^{3+}$ ions with respect to residue sequence coverage, ion 

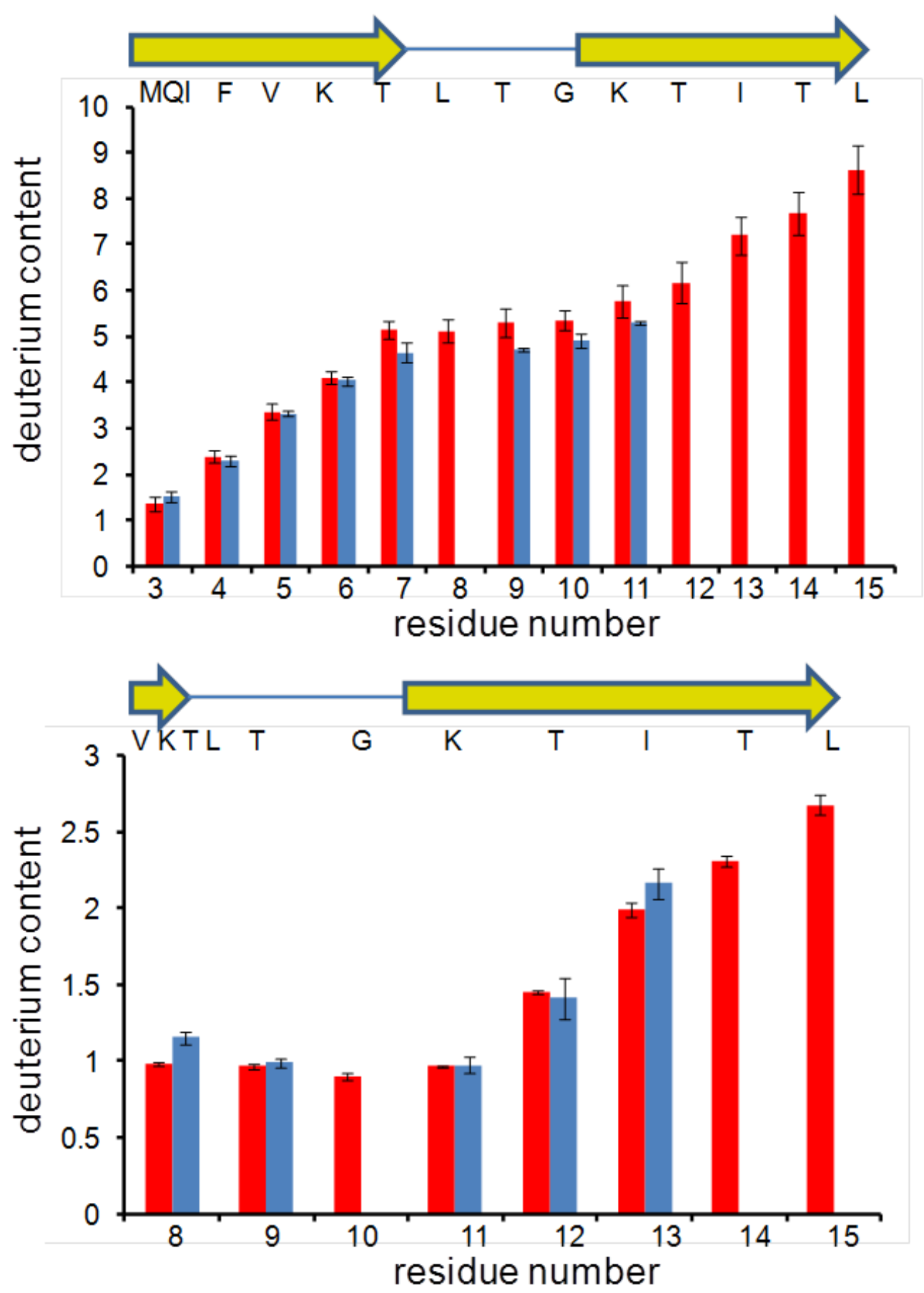

Figure 5.5 . (A) Bar plots show the total deuterium content per residue, $n$, calculated from the $c_{n-1}$ product ions generated by ETD (red traces) and He-CTD (blue traces) from labeled $[\text { MQIFVKTLTGKTITL }+3 \mathrm{H}]^{3+}$ ions. The $\mathrm{N}$-terminal region of ubiquitin spanning a beta strand (residues $\left.M^{1}-T^{7}\right)$, turn $\left(L^{8}-G^{10}\right)$ and second beta strand from $\left(G^{10}-L^{15}\right)$ is also shown. (B) Total deuterium content plot for residues $V^{5}-L^{25}$ generated by ETD (red traces) and He-CTD (blue traces) of labeled [VKTLTGKTITL $+3 \mathrm{H}]^{3+}$ ions. A section of the beta-stranded region and the second beta strand across residues $\mathrm{V}^{5}-\mathrm{T}^{7}$ and $\mathrm{G}^{10}-\mathrm{L}^{15}$, respectively, are also shown. The error was calculated from triplicate measurements of respective fragment ions. 
deuterium content levels and qualitative structural trends are very similar to previous studies.[14]

Also originating from the $\mathrm{N}$-terminal region, [VKTLTGKTITL+3H] $]^{3+}$ ions were investigated using DHX-PD-MS of labeled ubiquitin. Although the sequence overlaps significantly with [MQIFVKTLTGKTITL $+3 \mathrm{H}]^{3+}$ ions, pepsin digestion at the carboxyl side of $\mathrm{V}^{5}$ significantly changes the deuterium content level. Figure 5.6B shows the deuterium content for $c$ ions originating from [VKTLTGKTITL $+3 \mathrm{H}]^{3+}$ precursor ions after HDX-ETD-MS of labeled ubiquitin. Evaluation of Figure 5.6B shows a deuterium content level of $\sim 1$ for residue $T^{7}$ after HDX. A reduced deuterium level may be expected because much of the structured $\mathrm{N}$-terminal region has been cleaved. Enzymatic digestion also reacts to form a primary amine from the backbone amide of $\mathrm{V}^{5}$, which can exchange (even under quench conditions) and further reduces the deuterium content level. Residues $\mathrm{T}^{7}-\mathrm{G}^{10}$ show no change in deuterium content, follow by an increase across residues $\mathrm{K}^{11}-\mathrm{L}^{15}$. This trend is similar to that of [MQIFVKTLTGKTITL+3H] ${ }^{3+}$ ions (see above) and also appears to correctly map structured areas within ubiquitin.

\subsubsection{Per-Residue He-CTD-MS Analysis}

In order to provide direct comparisons between ETD and He-CTD fragment data for structural analysis, [MQIFVKTLTGKTITL+3H] ${ }^{3+}$ ions generated during HDX-MS were also selected for He-CTD experiments. Figure 5.6A shows the deuterium content level for $c$ ions resulting from HDX-PD-He-CTD-MS. Evaluation of the deuterium content appears to begin at residue $\mathrm{I}^{3}$ and sequentially increases to $\mathrm{T}^{7}$. Interestingly, the deuterium content levels for these ions appear to be similar to the levels determined from ETD experiments. For example, the deuterium content for residues $T^{7}-T^{9}$ appears 
unchanged. This is consistent with the unstructured turn between beta-sheets as mentioned above. Although the deuterium content level is slightly lower than that the determined by ETD, it is noteworthy that this region could have exchanged out to a higher degree before He-CTD studies. The highly structured region between $\mathrm{I}^{3}-\mathrm{K}^{6}$ shows similar deuterium content levels between ETD and He-CTD. A slight increase in deuterium content is observed for residue $\mathrm{K}^{11}$ relative to $\mathrm{T}^{9}$. This trend is similar to that for the ETD analysis, where a small increase in deuterium content was observed for $\mathrm{K}^{11}$. Together, these similarities suggest that He-CTD is capable of qualitatively determining areas of structure within labeled proteins and this further indicates that HD-scrambling is largely avoided.

Figure 5.6B shows the $c$ ions resulting from HDX-He-CTD-MS of labeled $[\text { VKTLTGKTITL+3H] }]^{3+}$ ions. A very similar trend to that observed for ETD is noted for these ions. Residue $\mathrm{T}^{7}$ retains $\sim 1$ deuterium, which may be expected given the second peptic cleavage event between residues $\mathrm{F}^{4}$ and $\mathrm{V}^{5}$. As mentioned above, digestion converts the backbone amide to a primary amine that subsequently allows $\mathrm{K}^{6}$ to be accessible to exchange, further reducing the deuterium content. Reduced deuterium content has been reported in other peptides using pepsin digestion HDX-MS experiments.[21] The deuterium content level is unchanged across the unstructured region $\left(T^{7}-T^{9}\right)$ and appears to increase from $G^{10}-T^{12}$. Residue $G^{10}$ has a slightly lower deuterium content level reported for $\mathrm{G}^{10}$ from [MQIFVKTLTGKTITL $\left.+3 \mathrm{H}\right]^{3+}$ product ion determinations. Because this residue is located at the edge of the second beta-sheet, decreased protection may be expected.[20] However, other consistencies are noted. For example, the deuteration content increases at $\mathrm{K}^{11}$ and is comparable with ETD data 
(Figure 5.6B) as well as both experiments for [MQIFVKTLTGKTITL $+3 \mathrm{H}]^{3+}$ ions (Figure 5.6A). Another consistency between ETD and He-CTD is the lower deuterium content level across the unstructured region, before the second beta-sheet, which was noted in Figure 5.6A.

Evaluation of both peptide ions reveals that He-CTD results in sequence coverage across residues $\mathrm{M}^{1}-\mathrm{I}^{13}$ and allows for a qualitative view of secondary structure across the $\mathrm{N}$-terminal region. For example, retention for the $\mathrm{L}^{8}$ residue was not observed in the He-CTD spectrum for [MQIFVKTLTGKTITL+3H] ${ }^{3+}$ ions; however, data for the $L^{8}$ residue (Figure 5.6B) shows that deuterium content level is unchanged across residues $L^{8}-T^{9}$. As mentioned above, this observation is consistent with the unstructured turn between beta-sheets. Additionally, Figure 5.6B shows that deuterium content increases from $T^{11}-I^{12}$ which was also not observed upon He-CTD of $[\text { MQIFVKTLTGKTITL }+3 H]^{3+}$ ions. These residues are located within the second betastrand and are expected to display a level of protection. Although He-CTD is currently not as efficient as ETD, the analysis from both precursor ions gives $\sim 90 \%$ sequence coverage across the $\mathrm{N}$-terminal region of ubiqutin. Notably, these data are consistent with the known secondary structural elements within ubiquitin. Given the comparison between ETD and the resulting spectral consistencies, the compilation of these data suggest that HD-scrambling for is largely mitigated upon He-CTD.

\subsection{Conclusion}

Using ETD as the gold standard technique for per-residue HDX studies and a model peptide specifically designed to monitor HD-scrambling, separate experiments show that He-CTD generated $c$ ions preserve the solution-phase deuterium label. 
Generated $c$ ions via ETD were used in a statistical analysis to determine instrumental parameters that resulted in $0 \% \mathrm{HD}$-scrambling. Using these determined conditions, HDX-He-CTD-MS of $[\mathrm{M}+3 \mathrm{H}]^{3+}$ labeled MP produced a series of $c$-ions that were compared to c-ions produced during ETD studies. Fragment c-ions produced by $\mathrm{He}$ CTD were found to mirror a $0 \% \mathrm{HD}$-scrambling trend based on theoretical ETD calculations. Because He-CTD efficiency is relatively low, a homologous series of either ion series was not generated. However, a complementary HDX-He-CTD-MS scrambling study using deammoniated product ions also revealed that HD-scrambling is mitigated. HD preservation was observed via the neutral loss of ammonia that was absent the incorporation of deuterium from labeled regions of the peptide. Using the determined non-scrambling conditions,

As a proof-of-concept study, a system for online HDX and pepsin digestion (PD) was combined with He-CTD for structural analysis of deuterium-labeled ubiquitin. Ions of highest abudance were selected and analyzed by ETD-MS and He-CTD-MS. Deuterium retention calculated from $c$-ions across residues $\mathrm{M}^{1}-\mathrm{L}^{15}$ and $\mathrm{V}^{5}-\mathrm{L}^{15}$ are in good agreement with structural trends found in pre-residue studies using ubiquitin.[3, 14, 19] Lastly, ETD-MS and He-CTD-MS deuterium content level for the respective residues are highly similar. From these results it appears, at least for $c$-ions, that $\mathrm{He}$ CTD may be used as a new technique for DHX structural studies of proteins. 


\subsection{References}

1. Huang, R.Y.C., et al., Hydrogen/Deuterium Exchange and Electron-Transfer Dissociation Mass Spectrometry Determine the Interface and Dynamics of Apolipoprotein E Oligomerization. Biochemistry, 2011. 50(43): p. 9273-9282.

2. Pan, J. and C.H. Borchers, Top-down structural analysis of posttranslationally modified proteins by Fourier transform ion cyclotron resonance-MS with hydrogen/deuterium exchange and electron capture dissociation. Proteomics, 2013. 13(6): p. 974-81.

3. Pan, J., et al., Electron Capture Dissociation of Electrosprayed Protein Ions for Spatially Resolved Hydrogen Exchange Measurements. Journal of the American Chemical Society, 2008. 130(35): p. 11574-11575.

4. Landgraf, R., M. Chalmers, and P. Griffin, Automated Hydrogen/Deuterium Exchange Electron Transfer Dissociation High Resolution Mass Spectrometry Measured at Single-Amide Resolution. Journal of The American Society for Mass Spectrometry, 2012. 23(2): p. 301-309.

5. Rand, K.D., et al., Protein Hydrogen Exchange Measured at Single-Residue Resolution by Electron Transfer Dissociation Mass Spectrometry. Analytical Chemistry, 2009. 81(14): p. 5577-5584.

6. Wysocki, V.H., et al., Mobile and localized protons: a framework for understanding peptide dissociation. Journal of Mass Spectrometry, 2000. 35(12): p. 1399-1406.

7. Zehl, M., et al., Electron Transfer Dissociation Facilitates the Measurement of Deuterium Incorporation into Selectively Labeled Peptides with Single Residue Resolution. Journal of the American Chemical Society, 2008. 130(51): p. 17453-17459.

8. Rand, K.D., et al., Electron capture dissociation proceeds with a low degree of intramolecular migration of peptide amide hydrogens. J Am Chem Soc, 2008. 130(4): p. 1341-9.

9. Rand, K.D., et al., Protein hydrogen exchange measured at single-residue resolution by electron transfer dissociation mass spectrometry. Anal Chem, 2009. 81(14): p. 5577-84.

10. Rand, K.D., et al., Loss of Ammonia during Electron-Transfer Dissociation of Deuterated Peptides as an Inherent Gauge of Gas-Phase Hydrogen Scrambling. Analytical Chemistry, 2010. 82(23): p. 9755-9762.

11. Rand, K.D., M. Zehl, and T.J. Jorgensen, Measuring the hydrogen/deuterium exchange of proteins at high spatial resolution by mass spectrometry: overcoming gas-phase hydrogen/deuterium scrambling. Acc Chem Res, 2014. 47(10): p. 3018-27.

12. Hoffmann, W. and G. Jackson, Charge Transfer Dissociation (CTD) Mass Spectrometry of Peptide Cations Using Kiloelectronvolt Helium Cations. Journal of The American Society for Mass Spectrometry, 2014. 25(11): p. 1939-1943.

13. Chingin, K., et al., Fragmentation of Positively-Charged Biological lons Activated with a Beam of High-Energy Cations. Analytical Chemistry, 2014. 86(1): p. 372-379.

14. Donohoe, G.C., J.R. Arndt, and S.J. Valentine, Online Deuterium Hydrogen Exchange and Protein Digestion Coupled with lon Mobility Spectrometry and Tandem Mass Spectrometry. Analytical Chemistry, 2015. 87(10): p. 5247-5254.

15. Cook, S.L., O.L. Collin, and G.P. Jackson, Metastable atom-activated dissociation mass spectrometry: leucine/isoleucine differentiation and ring cleavage of proline residues. Journal of Mass Spectrometry, 2009. 44(8): p. 1211-1223.

16. Misharin, A.S., et al., Dissociation of peptide ions by fast atom bombardment in a quadrupole ion trap. Rapid Communications in Mass Spectrometry, 2005. 19(15): p. 2163-2171.

17. Johnson, E.C., et al., Solution structure and dynamics of a designed hydrophobic core variant of ubiquitin. Structure, 1999. 7(8): p. 967-976.

18. Bougault, C., et al., Quantitation of rapid proton-deuteron amide exchange using hadamard spectroscopy. Journal of Biomolecular NMR, 2004. 28(4): p. 385-390. 
19. Sterling, H.J. and E.R. Williams, Real-Time Hydrogen/Deuterium Exchange Kinetics via Supercharged Electrospray Ionization Tandem Mass Spectrometry. Analytical Chemistry, 2010. 82(21): p. 9050-9057.

20. Kaltashov, I.A., C.E. Bobst, and R.R. Abzalimov, H/D Exchange and Mass Spectrometry in the Studies of Protein Conformation and Dynamics: Is There a Need for a Top-Down Approach? Analytical Chemistry, 2009. 81(19): p. 7892-7899.

21. Percy, A.J., et al., Probing protein interactions with hydrogen/deuterium exchange and mass spectrometry-a review. Analytica chimica acta, 2012. 721: p. 7-21. 


\section{Future Directions: Applied 'Omics using IMS-MS Techniques}

\subsection{Post Translational Modifications: Phosphorylation}

The IMS-CID-MS method was capable of determining the presence of phosphoserine and subsequently elucidating the site of modification. One advantage over traditional techniques was the ability to match product ions to respective precursors ions based on XIDTDs. Much of the matching capability is attributed high reproducibility of the mobility separation. Although, the experiment presented a proof-ofconcept study, only a single phosphopeptide was considered. Future studies will conduct IMS-CID-ETD-MS experiments for a wider range of phosphopeptides. Here, it is noted that threonine and tyrosine are also capable of phosphorylation. Some studies have indicated that the fragmentation energetics and observed neutral losses are different for each phosphorylated residues.[1] This presents some challenges in determining a collision energy that is amendable across all phophorylation possibilities. However, the ion activation region within the drift tube is highly tunable. Determining multiple ion activation voltages that can be stepped according to mobility is necessary for high throughput workflows.

\subsubsection{Doubly - Phosphorylated Peptides from Complex Mixtures}

With these critiques in mind, future experiments should not only address studies for a wider range of phophorylated residues, but also residues that are doubleymodified. Some research has shown that hyper-phosphorylation is important for tauopathies.[2] Highly phosphoryalted peptides may present the chance to observe unique fragment ions that are difficult to detect with traditional techniques. One difficulty 
in detecting doubly phosphorylated peptides is that fragmentation may result in the neutral loss from both or a single residue. With traditional techniques using QqQ instrumentation, the last quad would have to be scanned quickly across several potential fragment ions to determine the presence of phosphorylation. Because many product ions are possible, selecting the correct fragment ion with high specificity would be difficult. As stated previously, one potential benefit may come from the ability to perform spectral matching based on XIDTDs where all product ions can be spectrally aligned back with the precursor ion.

\subsubsection{Conducting IMS-CID-MS on Glycosylated Proteins}

As a brief overview, glycosolation is a covalent post-translational modification occurring through enzymatic mechanisms often occurring within the golgi apparatus after protein synthesis at the ribosome.[3] Glycosylation is important for cellular recognition[4] and protein folding.[5] There are two classes of glycosidic bonds formed at the amide nitrogen of asparagines having a consensus sequence Asn-Xxx-Ser (where $x$ cannot be proline) and at the hydroxyl oxygen of serine, tyrosine, and threonine. These respective classes are referred to as $\mathrm{N}$ - [6] and O-linked[7] glycosolyation. Glycans themselves are complex structures consisting of branched, or unbranched, networks of oligosaccharides that are arranged through $\beta 1-6$ or $\beta 1-4$ glycosidic linkages.

Glycan structural elucidation is much more difficult than that described for peptides or proteins. Common MS/MS methods for glycan structural analysis use CID and UVPD to produce glycosidic cleavage ions.[8] It is noteworthy to mention that 
GlcNAc and mannose are common moieties in glycan structures that produce fragment ion $\mathrm{m} / \mathrm{z}$ values of $201,202,203$ and 179 respectively. These fragment ions have been reported from $[\mathrm{M}-\mathrm{H}]^{-},[\mathrm{M}+\mathrm{H}]^{+}$and $[\mathrm{M}+\mathrm{Na}]^{+}$precursor ions form ESI of non-reduced sugars.[8-10] Future experiments could use protein digests to rapidly scan for reporter fragment ions common for glycosolation (e.g. m/z 202 or 179). Using XIDTDs, the fragment ions can be related back to their respective precursor ion. Once matched, the precursor ions could then be mobility selected and isolated in the ion trap for ETD-MS. Since ETD retains PTMs, the modified residue could then be determined.

As described, the IMS-CID-ETD-MS method could potentially be used to scan for the presence of glycosolyation events and identify modified residues. Because glycans are structurally diverse, another future experiment could attempt identifying the glycan from the modified protein. For these experiments, it is noted that linear ion traps suffer from low mass accuracy where high mass accuracy is often necessary for glycan identification. Considering this, the IMS-CID-ETD-MS method could offer potential targets for data dependent acquisition on mass spectrometers that achieve much higher mass accuracies $( \pm 2 \mathrm{ppm})$. This may allow glycan identification based solely on high mass accuracy and library searching against known glycans.[3] Another possibility may be to use RNase for deglycosolyation coupled with offline lectin affinity chromatography[11] or electrophoresis to isolate and purify glycans prior to MS analysis.[12] In this manner, glycans could be subjected to $\mathrm{MS}^{n}$ and their structures elucidated. Although this type of workflow would be lengthy, it combines the advantages of multiple platforms for increased structural detail.

\subsection{Expanding the Usage of IMS-CID-MS in Metabolomic Workflows}


The metabolite experiments presented in Chapter 2 were aimed at comparative metabolomic analysis. After PCA and $\mathrm{MS}^{\mathrm{n}}$ the analyses found that lipids could be used to differentiate the sample cohorts. This allowed unique parallel CID analyses to be performed for rapid data independent workflows and identification. From these data it was observed that many ions were identified from lysophosphatidylcholines (LysoPC) precursors. In part this may be attributed to ESI bias towards LysoPCs. Across the twodimensional $t_{D-} \mathrm{m} / \mathrm{z}$ plot, it appears that this lipid class is oriented to occupy related conformation space. Such observations have also been shown by McLean and coworkers using a high-resolution drift tube. In these experiments the IMS-MS results suggested unique intrinsic molecular packing from four separate molecular classes (carbohydrates, lipids, quanternary ammonium salts and peptides).[13] Although such results represent the start of IMS-MS libraries for more accurate determinations, it is noted that these experiments can be extended by adding IMS-CID-MS.

McLean's conformational analysis of $>300$ lipid standards comprised of glucosylceramides, phosphatidylcholines, phosphatidylethanolamines, phosphatidylserine and sphingomyelin resulted in $\mathrm{He}_{(\mathrm{g})}$ CCS values, which ranged from $220-350 \AA^{2}$ over a $m / z$ range of $800-1000$.[13] These results suggest that lipids display similar intrinsic conformations in the gas phase. Theoretical metabolomic studies could benefit by matching mobility information with conformational libraries for qualitative analysis purposes. Given the similar CCS values across lipid classes, it still remains difficult to identify a specific lipid class. Even smaller lipids, such as LysoPCs ( $m / z \sim 500)$, shown in Chapter 2, demonstrated distinct drift times indicative of similar CCS values (although not reported). 
Future experiments could be directed towards using similar lipid standards for IMS-CID-MS analysis. The goal would be to used the enhanced analytical space afforded by the mobility separation for fragment ions. This would allow the apparent CCS and $\mathrm{m} / \mathrm{z}$ correlation between lipid classes to be broken. Similar experiments have been successfully applied to peptides. $[14,15]$ That is, fragment ions would have the same mobility as the precursor ion, but a better distinction between lipid classes would be made based on the $\mathrm{m} / \mathrm{z}$ space. Here unique fragmentation patterns would emerge across a lipid class, even if two classes overlapped along the mobility separation. One important consideration is that the method would scan for the head region of the lipids. Not only does the region identify the lipid class, it represents a reporter ion and thus offers a spectral 'fingerprint' for lipid structure and class. Another important consideration is that the head region most often contains the charge site of the precursor ion. It is recalled that in Chapter 2, that the most dominant spectral features upon IMS-CID or MS/MS of LysoPC ions resulted from dissociation of the head region.

The application of IMS-CID-MS methodology for determining fine structural variation of lipid classes, expands on the concept of conformational ordering of biomolecules through fragment ions. The idea of using IMS-CID-MS to break CCS vs $\mathrm{m} / \mathrm{z}$ correlations has been described for peptides.[16] It is felt, that this methodology would also expand mobility libraries for spectral matching and identification. It is noted that these libraries are in their infancy and the contributions of such results would be timely. These libraries are need for data independent workflows, where high specificity and high throughput are at the heart of the technique.

\subsection{Gas Phase HDX of Anionic Proteins}




\subsubsection{Comparing Cationic and Anionic HDX Deuterium Level: Structural Studies}

Results of Chapter 3 report fundamental studies aimed at understanding proteinanionic structures in the gas phase. Through complementary studies involving IMS and gas phase HDX, insights into different conformers could be ascertained. One interesting observation was shown with compact and elegonated conformations of ubiquitin that demonstrated different deuterium uptake values. This was attributed to the protein fold that allowed a greater exposure of exchangeable sites in elongated conformations that were otherwise inaccessible in relatively compact conformers. Other peptides contributiing mulitmeric species showed reduced uptake and may be used in the future to indicate binding site interactions.

Some studies have indicated both similar and dissimilar conformations can be observed based on the polarity during native ESI-IMS-MS of smaller proteins.[17] Future directions may be able to compare protein cationic and anionic HDX values for different gas phase conformations. Here CCS values, exchange profiles and molecular dynamics may be able to show where protein conformations are different. This may also allow us to understand which residues are important for maintaining protein tertiary structures and how protein structure changes in the gas phase. By comparing the exchange profiles of both polarities a better picture of protein structure in the gas phase may emerge. For example consider a protein that has undergone ESI in negative polarity mode. In connection with CCS values and molecular dynamics, the HDX profiles may show how a particular portion of the protein becomes unfolded due to Coulomb repulsion of charge sites that is not experienced by the native solution structure. However, if we compare the complementary structure in positive mode, for 
example, the HDX profiles and CCS values may not indicate any distruption of protein structure. Such results may direct better experimental conditions for determining more accurate CCS values and help future experiments determine the optimal polarity mode for CCS measurements.

\subsubsection{Binding Faces of Acidic Proteins Involved in Protein Aggregates}

An interesting area of research has been studying the binding interfaces of protein oligomers. Two candidate proteins that display a high number of acidic residues are $\alpha$-synuclien and beta amyloid which form protein aggregates in Parkinson's and Alzheimer's diseases respectively. Acidic residues, such as aspartic acid and glutamic acid have $\mathrm{pK}_{\mathrm{a}} \mathrm{s} \sim 2.0$, giving these residues a negative charge in solution. Given the argument that different polarities can induce structural changes, a gas phase HDX experiment of anions may be used to ascertain interstitial binding faces of oligomeric proteins. Future experiments could incubate $\alpha$ synuclien over a defined period of time to induce oligermization followed by native ESI in negative ion mode. The gas phase ions could then be labeled using negative gas phase HDX. A recent study by Arndt and coworkers showed reduced deuterium uptake for $\mathrm{Nt} 17$ heterodimer associated with the Huntington's protein in positive polarity mode (unpublished results). IMS coupled with molecular dynamics, the study revealed an anti-parellel arrangement of stacked $\alpha$ helicies that were involved into multimerization. A similar negative polarity method could benefit acidic protein studies that present oligomeric species.

\subsubsection{Gas Phase HDX and Anionic Glycans: Structural Considerations and Separation}

\section{$\underline{\text { Techniques }}$}


Lastly it is noted that glycans can be analyzed in negative polarity mode. Using IMS-MS, some studies have revealed that glycans display diverse structures in the gas phase. This is thought to be attritubed to different gas-phase conformers as well as isomeric species. A method using negative mode gas phase HDX may be able to help differentiate glycans based on drift profiles and HDX uptake. Glycans appear to be strong candidates for HDX based on the high numbers of hetero-atomic groups (e.g. $\mathrm{OH})$. Given that the HDX process is highly dependent on charge site location and proximal hetero-atomic sites, different HDX level could be expected based on glycan structure. Exchange distances have been optimized for cytochrome c conformers[18, 19] and peptide ion species.[20, 21] That is exchange occurs for species that are within threshold distances of charge sites and any accessible heteroatom may undergo exchange. For example, a branched moiety that is accessible for exchange based on a charge site proximity model, may be expected to undergo HDX. Comparatively, another glycan may have alternate branching resulting in decreased accessibility for exchange. It is also noted that the IMS is used as the isotopic labeling cell. That is, if glycan exchange profiles are different, they are no longer isobaric and can be separated base on the deuterated $\mathrm{m} / \mathrm{z}$ values. Such results may be capable of differentiating glycans in the gas phase and further understanding glycan structure.

\subsection{Solution - Based DHX Coupled with IMS-ETD-MS}

\subsubsection{Constructing a New Micro-fluidic Chip for On - Line Analysis}

For DHX-IMS-ETD-MS experiments, the on-line system was constructed using high efficiency mixing-Tees and unfused silica capillary interfaced to a hand-pulled ESI 
emitter tip. Future experiments could aim at constructing a micro-fludic system. Here, a three-layer chip could be built. The center layer would be capable of introducing protein and exchange solution to a common point where mixing would occur. Over a defined length of an inner channel, exchange could proceed over a defined (controlled) period of time. Orthogonal to the exchange channel, an inlet for ice-cooled pepsin digestion solutionwould tee into the exchange channel and quench the reaction. This would also simultaneously cause digestion over a determined length. An outlet for digested sample could lead at the ESI source. The bottom layer would act to jacket the second layer from the quench solution to the outlet for ESI. This layer would use a continuous flow of cooled solution $\left(\sim 0^{\circ} \mathrm{C}\right)$ that would insure the quench/digestion region was maintained at $\sim 0{ }^{\circ} \mathrm{C}$. Lastly the top layer would act to encase the other two layers. A chip based system would be much more controllable and reproducible than the fused silica capillary reported in Chapter 3.

\subsubsection{Increasing Sequence Coverage for Per-residue Studies}

Another future direction would be to increase the per-residue sequence coverage afforded by ion fragmentation. In part, many of the peptides observed in Chapter 4 were doubly-charged, making efficient fragmentation via ETD difficult. Recently it has been shown that nitrobenzyl alcohol can increase the charge states of proteins without altering protein solution structure or HDX kinetics.[22] Here, an inlet for the infusion of nitrobenzyl alcohol could follow the pepsin digestion region to aid in increasing the charge state of peptides. Termed a supercharging agent,[23] such methodolgy may be beneficially for ETD per-residue studies employing a bottom-up approach. 


\subsubsection{Evaluating Larger Proteins}

Chapter 4 studies used the small model protein ubiquitin to provide a proof-ofconcept study aimed at combining drift tube IMS to DHX workflows. It is noted that in order to fully test the capabilities of the on-line IMS-ETD-MS system, a larger protein would be needed. For example, monoclonal antibodies are composed of two heavy ( 50 kDa) and two amino acid light chains ( $25 \mathrm{kDa})$ linked together via disulfide-bonds around the Fc region. Collectively these proteins are $150 \mathrm{kDa}$ and would offer a much larger protein for study. It is also noted that pepsin cannot digest disulfide bonds. This may present other potential modifications to the microfludic chip (see above) that could include an electrochemical reduction region after the $\mathrm{DHX}$ region, or other inlet ports for tris(2-carboxyethyl)phosphine (TCEP) or iodoacetamide for disulfide bond reduction prior to pepsin digestion.

\subsubsection{Connecting Rapid Solution Structural Changes with Solution DHX and IMS-MS}

Future experiments may be able to use a chip-based system combined with DHX-IMS-ETD-MS to rapidly monitor protein structural transformations. Here a deuterated protein could be infused and combined with a denaturant $\left(\mathrm{H}_{2} \mathrm{O}\right.$ :methanol) at the mixing port. It is important to note that that infusion of methanol would change DHX kinetics, thus no kinetic experiments would be conducted. Instead, the goal would be to monitor global deuterium levels resulting from different solution conformations at a single time-point. Capturing these changes would be dependent on the timescale of the structural transition as well as the allowed exchange period. Some studies have used a similar device to study structural changes after mixing ubiquitin with $\mathrm{H}_{2} \mathrm{O}$ :methanol 
(40:60) solution. The study indicated 2 dominant separate structures that were present for $\sim 5$ minutes. [24] After this time, the more native conformation merged into a single altered conformation. These structures were monitored using DHX after a 4 minute exchange out period. Notably, the separate conformers displayed different deuterium retention values. With this in mind, future experiments could perform a similar study aimed at connecting solution conformations using $\mathrm{DHX}$ with different gas phase structures determined by IMS. Such results may be able of linking solution structural changes to gas phase structures based on deuterium retention and CCS values.

\subsection{Helium Charge Transfer Dissociation : Insights and Future Outlook}

The low efficiency of the CTD process, coupled with the low resolution of the QIT and the complexity of CTD fragmentation reduced the number of distinguishable $c$ ions in both HD-scrambling and structural studies. Future experiments will tailor the gas flow and emission energy of the cation beam to influence the efficiency and potentially the fragmentation characteristics in a more controlled fashion. Although not fully demonstrated in this study, CTD offers the ability to fragment lower charge state ions with high energy, radical-driven processes that would otherwise yield charge reduction during ETD. With this, future experiments would aim to fragment doubly- and singlycharged cations in order to determine deuterium content values. Such a method would significantly enhance pe -residue deuterium measurement and sequence coverage.

\subsubsection{Using He-CTD for Anionic Peptides and Proteins}

Future studies could also use He-CTD to fragment anionic peptides and proteins in the gas phase. Similar experiments have been reported for Xe cations irradiated with 
negatively-charged peptides. The resulting fragmentation mechanism, termed negative electron transfer dissociation (nETD), is the reverse of the ETD process. Because HeCTD was shown to produce fragment ions without HD-scrambling for cationic speices, perhaps the fragmentation technique could be used for anionic peptides and proteins. Indeed, part of the reason for exploring other fragmentation methodologies was to assess the possibility of combining such a technique with gas-phase HDX for perresidue studies.

\subsubsection{Evaluating Other Ilons for HD-Scrambling}

Lastly, He-CTD produced a series of a-ions that may be beneficial for per-residue deuterium determinations. These fragments appear to result from radically driven processes similar to those described for ultraviolet photodissoication. That is, a-type ions result from homolytic cleavage of the $\mathrm{C}-\mathrm{C}_{\alpha}$ bond to form $\mathrm{a}_{n}$ and $\mathrm{a}_{n}+1$ ions. These $a+1$ ions suggest that secondary dissociation of $b$-ions to form a-ions is not the dominant fragmentation pathway; however, such reactions cannot be ruled out. Future experiments may be able to determine if a-ions undergo HD-scrambling for use in structural studies.

\subsubsection{Other Potential Usages; He-CTD-IMS - MS}

Although, He-CTD results were proof-of-concept and are intended for qualitative comparisons, it is instructive to present a potential use of He-CTD for protein structural studies in which low charge states are more representative of native solution conditions.[25-27] Here, it is noted that much of IMS-MS measurements are directed at native-like conformations that may persist in the gas phase. Often, these native 
conformations are low charge states and difficult to fragment with electron driven fragmentation. It has also been shown that for large protein complexes, CID produces charge asymmetric dissociation.[28] Considering this, it may be fruitful to develop new fragmentation techniques that address these problems. Certainly future experiments could leverage the advantages of cationic fragmentation and combine the technique with IMS-MS to analyze native protein complexes. Such experiments may open the door to new 'omics' analyses. 


\subsection{Ref}

1. Palumbo, A.M., et al., Tandem mass spectrometry strategies for phosphoproteome analysis. Mass Spectrometry Reviews, 2011. 30(4): p. 600625.

2. Wang, J.Z., et al., Abnormal hyperphosphorylation of tau: sites, regulation, and molecular mechanism of neurofibrillary degeneration. J Alzheimers Dis, 2013. 33 Suppl 1: p. S123-39.

3. Zhu, Z.K. and H. Desaire, Carbohydrates on Proteins: Site-Specific Glycosylation Analysis by Mass Spectrometry, in Annual Review of Analytical Chemistry, Vol 8 , R.G. Cooks and J.E. Pemberton, Editors. 2015. p. 463-483.

4. Wolfert, M.A. and G.J. Boons, Adaptive immune activation: glycosylation does matter. Nature Chemical Biology, 2013. 9(12): p. 776-784.

5. Xu, C. and D.T.W. Ng, Glycosylation-directed quality control of protein folding. Nature Reviews Molecular Cell Biology, 2015. 16(12): p. 742-752.

6. Apweiler, R., H. Hermjakob, and N. Sharon, On the frequency of protein glycosylation, as deduced from analysis of the SWISS-PROT database. Biochim Biophys Acta, 1999. 1473(1): p. 4-8.

7. Zauner, G., et al., Protein O-glycosylation analysis. Biological Chemistry, 2012. 393(8): p. 687-708.

8. Ko, B.J. and J.S. Brodbelt, $193 \mathrm{~nm}$ Ultraviolet Photodissociation of Deprotonated Sialylated Oligosaccharides. Analytical Chemistry, 2011. 83(21): p. 8192-8200.

9. Ritchie, G., et al., Identification of $N$-linked carbohydrates from severe acute respiratory syndrome (SARS) spike glycoprotein. Virology, 2010. 399(2): p. 257269.

10. Zhu, F., et al., Mannose7 glycan isomer characterization by IMS-MS/MS analysis. J Am Soc Mass Spectrom, 2012. 23(12): p. 2158-66.

11. Alley, W.R., B.F. Mann, and M.V. Novotny, High-sensitivity Analytical Approaches for the Structural Characterization of Glycoproteins. Chemical Reviews, 2013. 113(4): p. 2668-2732.

12. Alley, W.R. and M.V. Novotny, Structural Glycomic Analyses at High Sensitivity: A Decade of Progress, in Annual Review of Analytical Chemistry, Vol 6, R.G. Cooks and J.E. Pemberton, Editors. 2013. p. 237-265.

13. May, J.C., et al., Conformational Ordering of Biomolecules in the Gas Phase: Nitrogen Collision Cross Sections Measured on a Prototype High Resolution Drift Tube Ion Mobility-Mass Spectrometer. Analytical Chemistry, 2014. 86(4): p. 2107-2116.

14. Koeniger, S.L., et al., An IMS-IMS analogue of MS-MS. Analytical Chemistry, 2006. 78(12): p. 4161-4174.

15. Hoadlund-Hyzer, C.S., J.W. Li, and D.E. Clemmer, Mobility labeling for parallel CID of ion mixtures. Analytical Chemistry, 2000. 72(13): p. 2737-2740.

16. Merenbloom, S.I., et al., IMS-IMS and IMS-IMS-IMS/MS for separating peptide and protein fragment ions. Anal Chem, 2006. 78(8): p. 2802-9.

17. Allen, S.J., A.M. Schwartz, and M.F. Bush, Effects of Polarity on the Structures and Charge States of Native-Like Proteins and Protein Complexes in the Gas Phase. Analytical Chemistry, 2013. 85(24): p. 12055-12061. 
18. Valentine, S.J. and D.E. Clemmer, H/D exchange levels of shape-resolved cytochrome $c$ conformers in the gas phase. Journal of the American Chemical Society, 1997. 119(15): p. 3558-3566.

19. Valentine, S.J. and D.E. Clemmer, Temperature-dependent H/D exchange of compact and elongated cytochrome $c$ ions in the gas phase. Journal of the American Society for Mass Spectrometry, 2002. 13(5): p. 506-517.

20. Khakinejad, M., et al., Combining ion mobility spectrometry with hydrogendeuterium exchange and top-down MS for peptide ion structure analysis. J Am Soc Mass Spectrom, 2014. 25(12): p. 2103-15.

21. Khakinejad, M., et al., Gas-Phase Hydrogen-Deuterium Exchange Labeling of Select Peptide Ion Conformer Types: a Per-Residue Kinetics Analysis. J Am Soc Mass Spectrom, 2015.

22. Sterling, H.J. and E.R. Williams, Real-time hydrogen/deuterium exchange kinetics via supercharged electrospray ionization tandem mass spectrometry. Anal Chem, 2010. 82(21): p. 9050-7.

23. Sterling, H.J. and E.R. Williams, Origin of Supercharging in Electrospray Ionization of Noncovalent Complexes from Aqueous Solution. Journal of the American Society for Mass Spectrometry, 2009. 20(10): p. 1933-1943.

24. Hoerner, J.K., H. Xiao, and I.A. Kaltashov, Structural and Dynamic Characteristics of a Partially Folded State of Ubiquitin Revealed by Hydrogen Exchange Mass Spectrometryt. Biochemistry, 2005. 44(33): p. 11286-11294.

25. Clemmer, D.E. and M.F. Jarrold, Ion Mobility Measurements and their Applications to Clusters and Biomolecules. Journal of Mass Spectrometry, 1997. 32(6): p. 577-592.

26. Shelimov, K.B. and M.F. Jarrold, Conformations, Unfolding, and Refolding of Apomyoglobin in Vacuum: An Activation Barrier for Gas-Phase Protein Folding. Journal of the American Chemical Society, 1997. 119(13): p. 2987-2994.

27. Shelimov, K.B., et al., Protein Structure in Vacuo: Gas-Phase Conformations of BPTI and Cytochrome c. Journal of the American Chemical Society, 1997. 119(9): p. 2240-2248.

28. Wysocki, V.H., et al., Surface-Induced Dissociation of Small Molecules, Peptides, and Non-covalent Protein Complexes. Journal of the American Society for Mass Spectrometry, 2008. 19(2): p. 190-208. 


\section{Appendix 1}

Optimized Parameters Used for DHX-IMS-ETD-MS

Nano-ESI Parameters

Spray voltage $(\mathrm{kV}): 1.9$

Desolvation Temperature $\left({ }^{\circ} \mathrm{C}\right)$ : no direct application

$\underline{\text { IMS Parameters }}$

Source Funnel 1 DC voltage (V): 136.8

Funnel 2 RF voltage (V): 136.8

IMS length (meters): 1.00

Buffer Gas (g): He

Drift Pressure (torr): 2.72

Drift Field $\left(\mathrm{V} \cdot \mathrm{cm}^{-1}\right): ~ 10$

Gate 1 Trapping Containment Voltage (V): 25.0

Gate $2(\mathrm{~V}): 7.5 \mathrm{~V}$

Buffer Gas Temperature (K): 300

Mass Spectrometer Parameters (LTQ Velos)

Ion Optics

Multipole 00 Offset (V): -2.00

Lens 0 Voltage $(\mathrm{V}):-3.00$

Multipole 0 Offset (V): -8.00

Lens 1 Voltage (V): -10.00

Gate Lens Voltage

Multipole 1 Offset (V): -12.00 
Multipole 1 Amplitude (V p-p): 300

Front Lens (V): -8.50 


\section{Appendix 2}

Optimized Parameters Used for DHX-He-CTD-MS

ESI Parameters

Spray voltage $(\mathrm{kV}): 4.5$

Desolvation Temperature $\left({ }^{\circ} \mathrm{C}\right): 100$

Instrumental Parameters: Bruker ETD AmaZon Quadrupole lon Trap

Capillary Exit (V): 50

Funnel 1

$\operatorname{RF}(\mathrm{V}): 130.0$

$\ln (\mathrm{V}): 50.0$

Out (V): 50.0

Lens (V): 15.0

Funnel 2

$\operatorname{RF}(\mathrm{V}): 130.0$

$\ln (\mathrm{V}): 12.0$

Out (V): 10.0

Lens (V): 3.3

Octopole

$\mathrm{RF}(\mathrm{V}): 75.0$

1 DC (V): 2.3

Partition (V): 1.7

$2 \mathrm{DC}(\mathrm{V}): 1.1$

Focus 1 
L1 (V): 0.5

L2 (V): -10.0

L3 (V): -6.0

L4(V): -6.0

Multipole:

$\mathrm{RF}(\mathrm{V}): 36.7$

$\mathrm{DC}(\mathrm{V}):-4.0$

Focus 4

L1(V): -4.0

L2(V): -5.0

L3(V): -75.0

Trap Drive: $40.0 \%$ 
Expanding the Applications of Ion Mobility Spectrometry and Mass Spectrometry in Integrative 'Omics Analyses

Gregory C. Donohoe

Dissertation submitted to the Eberly College of Arts and Sciences at West Virginia University in partial fulfillment of the requirements for the degree of

Doctor of Philosophy in Chemistry

C. Eugene Bennett Department of Chemistry

APPROVAL OF THE EXAMINING COMMITTEE

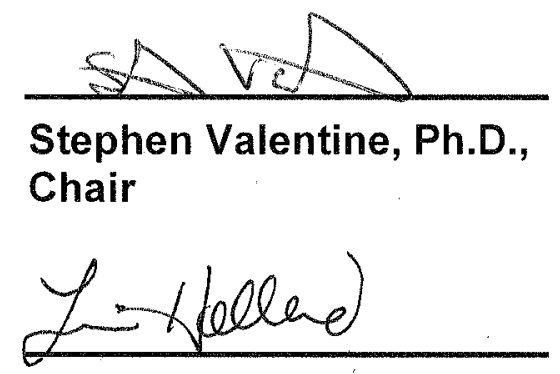

Lisa Holland, PhD.
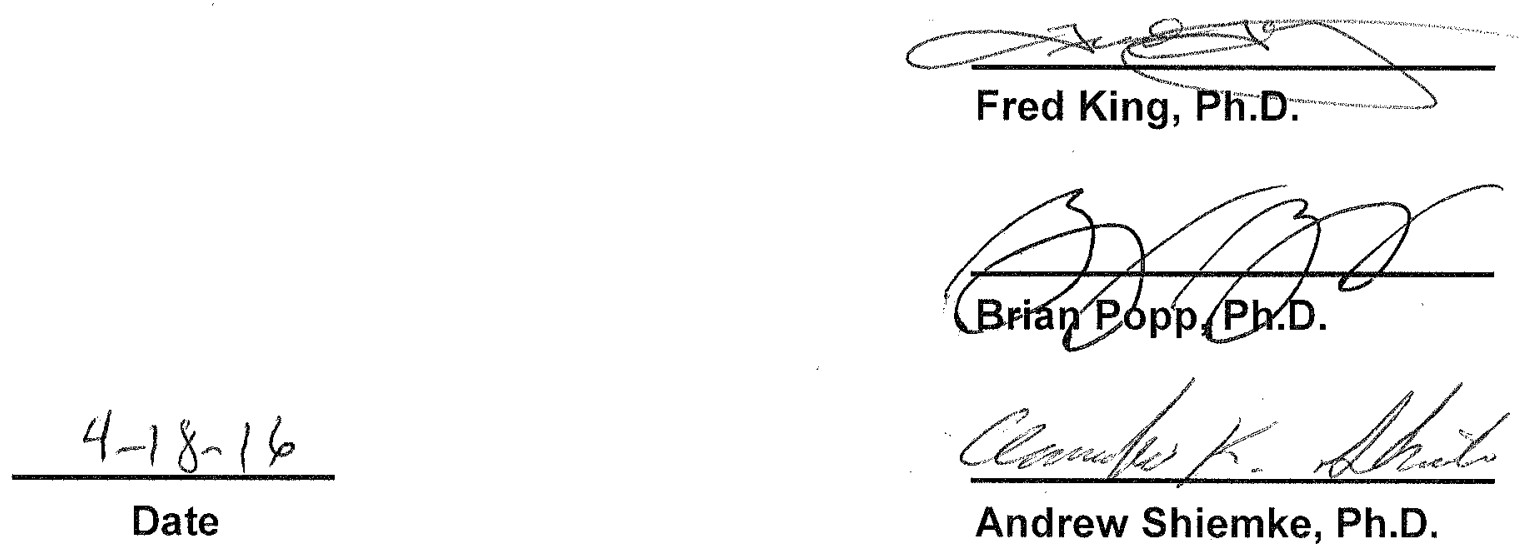

Andrew Shiemke, Ph.D. 